\title{
A STUDY OF THE PHOTOPRODUCTION OF THE
}

\section{$\Lambda_{c}^{+}$CHARMED BARYON AT $\gamma$ ENERGIES}

OF $40-160 \mathrm{GeV}$

by

Carl John Zorn

A Thesis submitted in conformity with the requirements

for the Degree of Doctor of Philosophy in the

University of Toronto

(C)Carl John Zorn

1986 


\section{ABSTRACT \\ A Study of the Photoproduction of the \\ $\Lambda_{c}^{+}$Charmed Baryon at $\gamma$ energies \\ of $40-160 \mathrm{GeV}$. \\ by}

Carl J. Zorn

Evidence for the $\Lambda_{c}^{+}$charmed baryon has been found in experiment E516 at the Tagged Photon Spectrometer in Fermilab. The experiment studied high energy $\gamma p$ interactions for photon energies in the range of $40-160 \mathrm{GeV}$ by utilizing a large acceptance spectrometer system to study the forward reaction products and a unique, sophisticated recoil chamber to study the target fragments.

The charm signal was detected in the decay channel $\Lambda_{c}^{+} \rightarrow \Lambda \pi^{+}$. The mean mass was measured as $2270 \pm 6 \mathrm{MeV} / \mathrm{c}^{2}$ with a full width at half maximum of $36 \pm 11$ $\mathrm{MeV} / \mathrm{c}^{2}$ - a measure of the experimental resolution. The world average for the $\Lambda_{c}$ mass is $2281.2 \pm 3.0 \mathrm{MeV} / \mathrm{c}^{2}$. A total of $51 \pm 14$ events constitute the signal with a statistical significance of 3.8 standard deviations.

The evidence indicates that the $\Lambda_{c}^{+}$is produced in association with the charmed $\bar{D}$ mesons by a non-diffractive mechanism:

(1) The antiparticle to the observed charmed particle, the $\bar{\Lambda}_{c}^{-}$baryon, was not observed in the data.

(2) The probability for $\Lambda_{c}^{+}$production peaks in the lower half of the photon beam energy spectrum. This is consistent with observations from previous photoproduction experiments at lower photon beam energies. 
(3) Both the rapidity and Feynman $x_{F}$ distributions peak at negative values. This is consistent with a theoretical model of the reaction $\gamma p \rightarrow \Lambda_{c}^{+} \bar{D}^{0} X$ using photon-gluon fusion and string fragmentation to generate the final state.

(4) Analysis of the target fragments in the recoil chamber supports the hypothesis of a non-diffractive mechanism.

Finally, the results of this experiment are an extension of previous observations in two ways. First, signal depletion of the charmed baryon signal is observed for photon energies above $100 \mathrm{GeV}$. Secondly, the unique presence of the recoil detector has allowed the observation of the non-diffractive nature of the production mechanism. 


\section{ACKNOWLEDGMENTS}

First, I would like to thank the other members of the E516 collaboration as a whole.

D. E. Blodgett, G. F. Hartner, B. R. Kumar, G. J. Luste,

J. F. Martin, K. K. Shahbazian, A. J. Stacey, and R. A. Sheperd

University of Toronto, Toronto, Ontario, Canada M5S 1A7

P. Estabrooks and J. Pinfold

Carleton University, Ottawa, Ontario, Canada K1S 5B6

M. J. Losty

National Research Council of Canada

Ottawa, Ontario, Canada K1A 0R6

G. R. Kalbfleisch and M. Robertson

University of Oklahoma, Norman, Oklahoma 73019

D. F. Bartlett, S. Bhadra, A. L. Duncan, J. R. Elliott, and U. Nauenberg

University of Colorado, Boulder, Colorado 80309

V. K. Bharadwaj, B. H. Denby, A. M. Eisner, R. G. Kennett, A. Lu

R. J. Morrison, D. J. Summers, M. S. Witherell, and S. J. Yellin University of California at Santa Barbara

Santa Barbara, California 93106

J. A. Appel, J. Biel, D. Bintinger, S. B. Bracker, J. Bronstein, C. Daum, P. M. Mantsch, T. Nash, M. V. Purohit, W. Schmidtke, K. Sliwa, M. D. Sokoloff, W. J. Spalding, K. C. Stanfield, M. Streetman, and S. E. Willis

Fermilab National Accelerator Laboratory

Batavia, Illinois 60510

A special thanks to my fellow graduate students with whom I have shared the unique pleasures and pains of graduate student life in experimental high energy physics: Dale, Bob, and Jim. And you too, Ian. Thanks to George, Don, Bruce, Ravi, and Gerd for reading the preliminary draft of this thesis. You have my sympathy. A further thanks to my supervisor George Luste-his advice and encouragement has made the completion of this work possible. I offer my gratitude to the National Science and Engineering Research Council of Canada, and the University of Toronto for providing highly valued and appreciated financial support during my graduate studies.

Thanks to Marilyn for being the best of friends. Finally, a special note of gratitude to my family in Ottawa for putting up with the one square egg in the clutch. 
Jaz hočem posvetiti to moje delo $v$ spomin moji preljubi materi Ivanki Vidi Arčon Zorn, ona je kri mojega srca. Prošnja $v$ moji molitvi je, da se še enega dneva srečava.

I dedicate this work to the loving memory of my mother, Ivanka Vida Arčon Zorn. She is the blood of my heart. I pray that we will meet again someday. 


\section{Table of Contents}

\section{Chapter 1: Introduction and Motivation.}

(1-1) What is the World Made of? . . . . . . . . . . . . . . . . . 1

(1-2) Charm Physics . . . . . . . . . . . . . . . . . . . . 4

(1-3) Photoproduction of the $\Lambda_{c}$. . . . . . . . . . . . . . . . . . . 6

(1-4) Experiment E516 . . . . . . . . . . . . . . . . . . . . . . 9

Chapter 2: Experimental Apparatus and Data Reconstruction.

(2-1) Outline . . . . . . . . . . . . . . . . . . . . . . 12

(2-2) The Proton Accelerator Facility . . . . . . . . . . . . . . . . 12

(2-2-1) Pre-accelerator . . . . . . . . . . . . . . . . 12

(2-2-2) Linac . . . . . . . . . . . . . . . . . . 13

(2-2-3) Booster . . . . . . . . . . . . . . . . 14

(2-2-4) Main Ring . . . . . . . . . . . . . . . . . 14

(2-2-5) Beam Extraction and Utilization . . . . . . . . . . . . . 15

(2-3) Tagged Photon Beam Line . . . . . . . . . . . . . . . . . . 17

(2-3-1) Electron Beam Line . . . . . . . . . . . . . . . . . . . 17

(2-3-2) Tagging System . . . . . . . . . . . . . . 18

(2-4) Tagged Photon Spectrometer . . . . . . . . . . . . . . . . 21

(2-4-1) Hydrogen Target . . . . . . . . . . . . . . . . . . . 21

(2-4-2) Recoil Spectrometer . . . . . . . . . . . . . . . . . . . 21

(2-4-2a) Construction Aspects of the Recoil Detector . . . . . . . . 24

(2-4-2a(i)) Proportional Wire Chambers . . . . . . . . . . . 24

(2-4-2a(ii)) Recoil Calorimeter . . . . . . . . . . . . . . 25

(2-4-2b) Recoil Calibration . . . . . . . . . . . . . . 26

(2-4-2c) Recoil Reconstruction . . . . . . . . . . . . . . 28 
(2-4-3) Analyzing Magnets . . . . . . . . . . . . . . . . . . . . 31

(2-4-4) Drift Chambers . . . . . . . . . . . . . . . . . . . . . 32

(2-4-4a) Drift Chamber Calibration . . . . . . . . . . . . . . 34

(2-4-4b) Charged Track Reconstruction _. . . . . . . . . . . . . 36

(2-4-4c) Primary and Secondary Vertex Reconstruction . . . . . . 39

(2-4-5) Čerenkov Counters . . . . . . . . . . . . . . . . . . . . 41

(2-4-5a) Čerenkov Calibration . . . . . . . . . . . . . . . . . 43

(2-4-5b) Cerenkov Reconstruction and Particle Identification . . . . 44

(2-4-6) SLIC . . . . . . . . . . . . . . . . . . . . . 46

(2-4-6a) SLIC Calibration . . . . . . . . . . . . . . . . 48

(2-4-7) Outriggers . . . . . . . . . . . . . . . . . . . . . . . . 49

(2-4-7a) Electromagnetic Calorimetry Reconstruction . . . . . . . 51

(2-4-8) Hadrometer . . . . . . . . . . . . . . . . . . . 54

(2-4-8a) Hadrometer Reconstruction . . . . . . . . . . . . . . . 55

(2-4-9) Muon Counters . . . . . . . . . . . . . . . . . . . . . . 56

(2-4-9a) Muon Identification . . . . . . . . . . . . . . . . . . 57

(2-4-10) Data Acquisition System . . . . . . . . . . . . . . . 58

(2-4-10a) Low Level Trigger Logic . . . . . . . . . . . . . . . . 58

(2-4-10b) Data Logging and Monitoring . . . . . . . . . . . . .61

(2-4-11) Trigger Processor . . . . . . . . . . . . . . . . . .62

(2-4-12) The 168E Emulator System . . . . . . . . . . . . . 66

\section{Chapter 3: Data Analysis and Physics Discussion.}

(3-1) Introduction . . . . . . . . . . . . . . . . . . . . . . . . .69

(3-2) The $\Lambda$ Filter . . . . . . . . . . . . . . . . . . . . . . . . . 71

(3-3) The $\Lambda_{c}$ Search . . . . . . . . . . . . . . . . . . . . . . . . 76

(3-3-1) Distance of $\Lambda_{c}^{+}$from Main Vertex . . . . . . . . . . . . . . 79

(3-3-2) Cut on $\Lambda$ Mass . . . . . . . . . . . . . . . . . . . . . . . . . . . 79

(3-3-3) Well-Identified Recoil Proton . . . . . . . . . . . . . . . 79 
(3-3-4) Transverse Momenta of the $\Lambda_{c}^{+}$. . . . . . . . . . . . . . 80

(3-3-5) One $\Lambda$ per Event and a Well-defined Beam Energy . . . . . . 81

(3-3-6) Previous Observations of the Decay Mode $\Lambda_{c}^{+} \rightarrow \Lambda \pi^{+}$. . . . . 81

(3-4) Characteristics of the $\Lambda_{c}^{+}$Signal . . . . . . . . . . . . . . 82

(3-5) The E516 Monte Carlo . . . . . . . . . . . . . . . . . . . . 85

(3-6) Estimate of the $\Lambda_{c}^{+}$Cross Section . . . . . . . . . . . 88

\section{Chapter 4: Conclusions}

(4-1) Summary of Results . . . . . . . . . . . . . . . . . . . . 94

(4-2) Comments on Charmed Baryon Physics . . . . . . . . . . . . 95

\section{Appendix A}

Some Kinematics of the $\Lambda$ Decay . . . . . . . . . . . . . . 97

\section{Tables}

Chapter 1 . . . . . . . . . . . . . . . . . . . . . . . 99

Chapter 2 . . . . . . . . . . . . . . . . . 105

Chapter 3 . . . . . . . . . . . . . . . . . . . . 107

\section{Figures}

Chapter 1 . . . . . . . . . . . . . . . . . . . 111

Chapter 2 . . . . . . . . . . . . . . . . . . . . . . . . . . . . 116

Chapter 3 . . . . . . . . . . . . . . . . . 133

References ..................... 155

Afterword . . . . . . . . . . . . . . . 164 
"I'll make my report as if I told a story, for I was taught as a child on my homeworld that Truth is a matter of the imagination. The soundest fact may fail or prevail in the style of its telling: like that singular organic jewel of our seas, which grows brighter as one woman wears it and, worn by another, dulls and goes to dust. Facts are no more solid, coherent, round, and real than pearls are. But both are sensitive.

The story is not all mine, nor told by me alone. Indeed I am not sure whose story it is; you can judge better. But it is all one, and if at moments the facts seem to alter with an altered voice, why then you can choose the fact you like best; yet none of them are false, and it is all one story." 


\section{CHAPTER 1}

\section{INTRODUCTION and MOTIVATION}

\section{(1-1) What is the World Made of?}

Physics is the study of matter and energy. Particle physics carries on this study at its most fundamental level. The answer to the above question has changed many times in many cultures down through the centuries and will no doubt continue to do so. Nevertheless the present viewpoint offers a rich and varied tapestry of ideas concerning the fundamental constituents and their interactions.

These constituents can be classified into two sets: one comprising the basic building blocks of matter from which all other forms of matter are constructed, and the other set contains those particles which mediate the interactions between members of the first set. The first set is further divided into two subsets of fermionsleptons and quarks. The lepton class is made up of the electron $(e)$, muon $(\mu)$ and tau $(\tau)$ along with their corresponding massless neutrinos $\left(\nu_{e}, \nu_{\mu}, \nu_{\tau}\right)$. Similarly there are three generations of quark doublets $(u, d),(c, s)$, and $(t, b)$ from which the spectrum of mesons and baryons can be constructed. No evidence for the existence of the $t$ quark is available as of this time. Table 1-1 displays some of the intrinsic characteristics of these particles.

In contrast, the particles that mediate the interactions among leptons and quarks are spin 1 objects called gauge bosons. There are four types of interactions, all of varying strengths: strong, electromagnetic, weak and gravitational. The strong interaction is mediated by eight types of particles called gluons and only occurs between quarks. In fact, it is thought that this force is strong enough to maintain quark confinement such that we can only observe the composite meson and baryon states. The electromagnetic force, mediated by the photon, can be exchanged among any of the electrically charged particles. Within the present orthodoxy, electromagnetism is interlinked with the weak force. This interaction is also 
possible among all the fundamental fermions. Unlike electromagnetism, which has an infinite range (hence implying that the photon must be massless), the weak force is short range $\left(\approx 10^{-18} \mathrm{~m}\right)$ and so is mediated by three massive bosons: $W^{ \pm}, Z^{0}$. These vector bosons have recently been found [117]. Gravitational interactions are of no importance within particle physics unless one is dealing with astrophysics and cosmology. In any case, no successful quantum theory of gravity has been formulated to date, although there are some promising ideas in development [118].

In mathematical terms, particle physics is described by Quantum Field Theory (QFT). Within this context, the fermion constituents are described by field wave functions whose modulus is proportional to the number density of particle states. An appropriate combination of these fields plus their derivatives with respect to space-time forms a Lagrangian from which the basic equations of motions can be derived. Note that the bosonic interaction fields are missing at this point. It is here that a crucial aspect of modern particle physics is displayed. By requiring that the Lagrangian remain invariant under a group of local gauge transformations, it is discovered that such a requirement forces the introduction of additional gauge fields to be introduced into the Lagrangian.

This principle can be used to formulate the theories of Quantum Electrodynamics (QED) and Quantum Chromodynamics (QCD). For the former, the Lagrangian is invariant under $U(1)$ while the latter is left invariant under $S U(3)$. The number of gauge fields equals the number of generators for the group. Hence electromagnetism has one field while QCD has eight fields.

Some qualifying remarks should be made. Since these gauge fields are massless, they are of no use in describing the weak interactions. At this point, the second aspect of modern particle theory is brought out. Usually, the Lagrangian will contain a term multiplied by a coefficient that is associated with the squared mass of the field. If we consider this just as a parameter, one finds some interesting results when this quantity is allowed to vary over both positive and negative values. 
When positive, it is seen that the ground state corresponds to the usual vacuum state expected. However, when the mass parameter passes into negative values, the vacuum state becomes a local maximum and the actual ground state corresponds to a set of non-zero values of the field. In this case, the Lagrangian will possess a symmetry not shared by the ground state. Hence a symmetry transformation which leaves the Lagrangian invariant will not leave the ground state invariant, but instead, will transform it into one of the other degenerate ground states. It is said that the system has a symmetry that is spontaneously broken. As a result of spontaneous symmetry breaking (SSB), one obtains a new set of massless, scalar fields called Goldstone bosons. When the SSB algorithm is applied to Lagrangians that are invariant under local gauge transformations, one finds that these Goldstone bosons are absorbed as new degrees of freedom by the gauge fields, resulting in the transformation of massless gauge fields into massive gauge bosons. Finally, one of the Goldstone bosons will also remain, but as a massive state. In this case, it is called the Higgs boson.

Both the gauge principle and the SSB mechanism are applied in unifying the weak and electromagnetic interactions. The resultant theory has come to be known as the Standard Model or alternatively as the Weinberg-Glashow-Salam Theory. The weak interaction part of the Lagrangian will transform under $S U(2)$ while the electromagnetic part is symmetric under $U(1)$. Overall, the symmetry group is the direct product group $S U(2) \otimes U(1)$. The number of gauge fields corresponds to the number of generators of the group. Hence, there are three fields for the weak and one field for the electromagnetic interaction. A scalar doublet field is then added to the Lagrangian and the principle of SSB applied. These four real fields are absorbed by the gauge fields in such a way as to create three massive fields mediating the weak force $\left(W^{ \pm}, Z^{0}\right)$ and the massless photon $(\gamma)$ mediating electromagnetism. As previously noted, one of the scalar fields remains, but is transformed into the massive Higgs boson. No evidence for its existence has yet been compiled. 


\section{(1-2) Charm Physics}

The original formulation of the quark model only included the up, down and strange $(u, d, s)$ quarks. The three quarks transformed under an approximate $S U(3)$ strong interaction symmetry (having nothing whatsoever to do with the exact $S U(3)$ colour symmetry) which was clearly broken by the differing quark masses. In weak interactions, it was supposed that the $u$ quark coupled to the combination $d^{\prime}=d \cos \theta_{c}+s \sin \theta_{c}$ where $\theta_{c}$ denoted the Cabibbo angle. Although this parameter is not understood at a fundamental level, it does account for many features of the $\Delta S=1$ and $\Delta S=0$ weak transitions. The point here is that with this manner of coupling, one should be able to see strangeness changing neutral transitions whereas the experimental facts show that this mode of decay is highly suppressed. For example, the branching ratio for $K_{L}^{0} \rightarrow \mu^{+} \mu^{-}$is $(9.1 \pm 1.9) \times 10^{-9}$. The charm quark hypothesis provides a way out of this dilemma.

In 1964, Bjorken and Glashow [111] proposed the existence of the charm quark as providing for a more symmetrical situation between quarks and leptons. (This symmetry is still adherred to in the present model.) But in 1970, Glashow, Iliopoulos and Maiani (the GIM paper) [112] revived this idea and more importantly, showed that the presence of this quark would eliminate the strangeness changing neutral current. As the $u$ couples to the $d^{\prime}$ state, so too does the charm quark $c$ couple to the combination $s^{\prime}=-d \sin \theta_{c}+s \cos \theta_{c}$. In the resultant expression for the neutral current, one obtains only diagonal terms, that is, there are no terms in which the quark flavour is changed. Strictly speaking, this result is only true at the first order level. Fortunately, as the charm quark mass is much smaller than that the $W$ bosons, the statement holds at the second order level as well.

The spectrum of possible particle states using four quarks can be displayed by making use of the approximate strong interaction symmetry among the quarks. In this case, the four quarks $(u, d, s, c)$ form the fundamental representation for the group $S U(4)$ (and the corresponding anti-quarks form the conjugate representa- 
tion). The meson states are $\left(q_{1}, \bar{q}_{2}\right)$ pairs while the baryons contain three quarks $\left(q_{1}, q_{2}, q_{3}\right)$. Hence, in the language of group theory, the meson states will form the basis for irreducible representations of the product of the fundamental and conjugate representations. Similarly, the baryons will involve the reduction of the triple product of the fundamental representation into its irreducible representations. The results are displayed in figure 1-1. Figures 1-1(a) and (b) display the $J^{P}=0^{-}$and $1^{-}$mesons respectively. Similarly the $\frac{1}{2}^{+}$and $\frac{3}{2}^{+}$baryons are illustrated in figures 1-1(c) and (d). The majority of evidence concerning the existence and properties of charmed states deals with the charmed mesons. In fact, the first sign of (hidden) charm was the famous simultaneous observation of the $J / \psi(3100)$ charmonium system $[109,110]$.

In contrast, relatively little evidence exists concerning the existence and production dynamics of the charmed baryons. There is certainly sufficient and significant evidence for the lowest mass charmed baryon, the $\Lambda_{c}$. As well, there is some data suggesting the existence of the $\Sigma_{c}, \Xi_{c}$ and the $\Omega_{c}$. Table 1-2 summarizes the current information concerning the charmed baryons. Note that the $\Lambda_{c}$ has two sets of mass values; one at about $2260 \mathrm{MeV} / \mathrm{c}^{2}$ and the more recent value of 2280 $\mathrm{MeV} / \mathrm{c}^{2}$. It has been suggested that this could indicate the existence a two-state diquark-quark system $[87,88]$. At present, the consensus is that the higher mass value is the most significant. Results published after the 1984 compilation do nothing to clear up this mystery. Aleev et al. [35], has reported a significant signal at $2268 \pm 6 \mathrm{MeV} / \mathrm{c}^{2}$. A similar value is reported in this thesis. In contrast, The CLEO group reports a value of $2287 \pm 11 \mathrm{MeV} / c^{2}$ [37] and the ARGUS collaboration reports a similar value [40]. Preliminary results from the experiment E691, the successor to the experiment reported in this thesis, indicate a very strong charm signal with high statistics, although, as of this writing, no well defined charmed baryon signal has been reported [130].

To find the charm particles, it is important to understand the dominant modes 
of decay. As noted above, the charm quark is coupled (weakly) to a linear combination of the strange and down quarks through the Cabibbo angle. Since $\theta_{c} \simeq 13^{\circ}$, the dominant amplitude will be a charm $\rightarrow$ strange transition with concomitant radiation of a $W^{+}$boson. The latter can couple to the $\left(u, \overline{d^{\prime}}\right)$ doublet where once again consideration of the Cabibbo angle indicates that the dominant pair will be $(u, \bar{d})$. Alternatively, the vector boson couples to one of the lepton douplets. Figure 1-2(a) illustrates the various possibilities and their likelihoods. In association with these diagrams, table 1-3 displays some characteristics of the decays in terms of quantum number changes. All of these modes involve the non-participation of any of the other quarks in the original particle state and is thus denoted as the spectator model. One can also have a $W^{+}$exchanged between the charm quark and one of the other valence quarks with the subsequent creation of a $(q, \bar{q})$ pair of sea quarks needed for the final hadronization of the decay. Figure 1-2(b) illustrates this process. Reference [88] ( $\&$ references therein) details the various final particle states possible in each of the two cases and discusses the requirements for separating out the contributions of the two processes. Experiment E691 may answer some of the questions posed.

For the purposes of this thesis the basic statement about non-leptonic charm decay is this: a charmed baryon will decay to a strange system containing one baryon plus one or more mesons. Illustrative of this fact are the dominant decays of the $\Lambda_{c}^{+}$:

$$
\begin{aligned}
& \Lambda_{c}^{+} \rightarrow K^{-} p \pi^{+} \\
& \bar{K}^{0} p \\
& (2.2 \pm 1.0 \%) \\
& (1.1 \pm 0.7 \%) \\
& \Lambda \pi^{+} \pi^{+} \pi^{-} \\
& (<3.1 \%) \\
& \Lambda \pi^{+} \\
& (0.6 \pm 0.5 \%)
\end{aligned}
$$

\section{(1-3) Photoproduction of the $\Lambda_{c}$}

Evidence for the existence of this charmed baryon has come mostly from fixed target experiments using neutrino and hadron beams plus the $e^{+} e^{-}$colliding beam exper- 
iments. The $\gamma p$ experiments have been conducted at SLAC, CERN and Fermilab. An argument can be made which suggests that an optimum search for and study of charm can be performed by photoproduction experiments [95].

In both the $e^{+} e^{-}$and photoproduction experiments, the photon (virtual in the $e^{+} e^{-}$case) couples to a $c \bar{c}$ pair through the charm quark electric charge $q_{c}$. The cross sections are proportional to $q_{c}^{2} / s$ and $q_{c}^{2} / m_{c}$ in $e^{+} e^{-}$and $\gamma p$ respectively (where $s$ is the center-of-mass energy and $m_{c}$ is the charm quark mass). It is estimated that about $40 \%$ of the $e^{+} e^{-}$and $1 \%$ of the $\gamma p$ cross sections should contain charm. In contrast, the relative charm component in hadroproduction should be an order of magnitude smaller. Since the absolute production rate for photoproduction is an order of magnitude higher than in $e^{+} e^{-}$collisions, one expects optimal charm production to occur in $\gamma p$ interactions. One has to qualify this statement as the realities of experimental design and its implementation can change the subsequent results. In fact, it is well known that the $e^{+} e^{-}$experiments have been very successful as a whole for studying charm production. Nevertheless there are some indications that this situation may change. As already noted, some preliminary results from E691 [130], the successor to E516, show that a very strong and clear charm signal is present in its data store.

Initially, charm photoproduction was interpreted in terms of the Vector Meson Dominance theory (VDM) [90]. Figure 1-3(a) displays this model in diagrammatic form. The photon, being a $J^{P}=1^{-}$state, couples to a virtual vector meson with the same quantum numbers. Below the charm threshold, these would be the $\rho, \omega, \phi$ particles; above the threshold, we have the $\psi$ family. This vector meson subsequently interacts with the proton through a vacuum state known as the Pomeron and produces the final state particles. Although this model has been successful in predicting the overall charm cross section, it has been replaced in recent years by a QCD-inspired model known as Photon-Gluon Fusion (PGF).

Figure 1-3(b) displays the first-order Feynman diagram in PGF for the inclusive 
reaction $\gamma p \rightarrow c \bar{c} X$. The second order processes are believed to have a negligible contribution to the final state. Another possible contribution to charm production concerns the intrinsic charm component of the proton. That is, there may be a pair of $c \bar{c}$ sea quarks within the proton that scatter off the photon to produce some charmed final states. Once again, it is believed to be insignificant relative to the PGF process [84].

In the paper by Fontannaz et al.[84], the authors make use of the PGF model to calculate the rapidity correlations between the final state charm particles. Following the creation of the $c \bar{c}$ pair, hadronization is carried out through string fragmentation. That is, a colour flux tube is set up between the $\bar{c}$ and a quark from the target nucleon, and between $c$ and the remaining diquark of the target nucleon. Hadronization takes place according to an assumed function which the authors found compatible with $e^{+} e^{-}$data. Figure 1-4 displays the application of PGF to the case study of reference [84]. The $\bar{c}$ quark interacts with a quark from the target proton to become a $\bar{D}^{0}$ meson. The other charm quark interacts with the remaining diquark to produce some non-charmed system $X$ and either the charmed $\Lambda_{c}^{+}$baryon or the charmed $D^{0}$ meson.

The rapidity $y$ is a useful parameter used in studying the production dynamics of a reaction and is defined as

$$
y=\frac{1}{2} \ln \left(\frac{E+P_{\|}}{E-P_{\|}}\right) .
$$

The quantities $E$ and $P_{\|}$are the energy and longitudinal momentum of the particle respectively. Although this quantity is dependent upon the frame of reference, it can be shown that the rapidities between two frames are related additively. Hence the rapidity distribution retains its shape under Lorentz boosts along the longitudinal momentum axis.

For the case of $\gamma p \rightarrow D^{0} \bar{D}^{0} X$ versus $\gamma p \rightarrow \Lambda_{c}^{+} \bar{D}^{0} X$ in the overall $\gamma p$ center-ofmomentum frame (CM), it was found that the rapidity distribution of the $\Lambda_{c}$ was peaked in the backward direction while the pair produced $D$ meson was emitted 
predominantly in the forward direction. This result is displayed in figure 1-5 for a photon energy of $100 \mathrm{GeV}$. Chapter 3 of this thesis contains a discussion of the relationship between this theoretical deduction and the experimental evidence presented therein.

\section{(1-4) Experiment E516}

Chapter 2 is a presentation of the experiment in its full glory. Nevertheless, this section should provide a useful précis. Indeed, the reader is advised to read chapters 3 and 4 after this chapter and then to read those portions of chapter 2 that are of interest and/or applicability.

Experiment E516 was designed to study charm events by triggering on high mass forward states formed in $\gamma p$ collisions. It used the set of detectors known as the Tagged Photon Spectrometer (TPS) located at the Fermilab accelerator facility. A portion of the $400 \mathrm{GeV}$ proton beam is used to create an electron beam with a tunable energy. Data with both a 137 and $170 \mathrm{GeV}$ electron beam was taken although the results in this thesis are based on the latter $170 \mathrm{GeV}$ data store. The $170 \mathrm{GeV}$ beam, with a typical flux of $4 \times 10^{6}$ electrons per one second pulse, was directed against a 0.2 radiation length copper target. Within the target, the electrons interacted with the copper nuclei through the bremsstrahlung process, thereby producing a photon beam. By bending the electrons into a lead glassscintillator hodoscope system, the energy of the corresponding photon could be measured (and hence tagged). The resultant photon energy spectrum covered a range of 40 to $160 \mathrm{GeV}$ with a mean energy of $105 \mathrm{GeV}$. The average flux was $7 \times 10^{5}$ photons per pulse.

This photon beam subsequently entered a liquid hydrogen target of 1.5 meters length which was placed in the center of the recoil spectrometer. This detector consisted of three concentric proportional wire chambers and four layers of scintillator. Tracking, time-of-flight and $d E / d x$ information from this detector was used to measure the 4-momenta of the recoiling particles. 
The charged particles proceeding into the forward part of the spectrometer were momentum analyzed by a system of two magnets and four assemblies of drift chambers comprising a total of 29 planes. Two unpressurized segmented Čerenkov counters were used to provide identification of the charged tracks. The upstream counter was filled with $N_{2}$ while the second contained a 22:78 $\mathrm{N}_{2} / \mathrm{He}$ mixture. The resultant thresholds allowed $\pi$ versus ( $K$ or $p$ ) discrimination in the range 6-20 $\mathrm{GeV} / \mathrm{c}$ and $\pi$ versus $K$ versus $p$ in the $20-36 \mathrm{GeV} / \mathrm{c}$ range.

At the downstream end of the spectrometer are electromagnetic and hadronic calorimeters for identifying electrons, neutral pions, neutrons and $K_{L}^{0}$. A final wall of scintillator following a meter of steel was used to identify muons.

The basic trigger required an hadronic interaction by the beam photons. This involved vetoing pair production, non-interactions and requiring energy deposits above a set threshold in the calorimeters. A high level, charm enhancing trigger was provided by a fast ECL-CAMAC trigger processor. This selected hadronic events consistent with having a single recoil proton at the production vertex and a missing mass between 2 and $11 \mathrm{GeV} / \mathrm{c}^{2}$. In practice, the trigger was split into two: Recoil 2 for the 2.0-5.5 GeV/ $\mathrm{c}^{2}$ mass region and Recoil 3 covers the high end from 5.5-11.0 GeV/ $\mathrm{c}^{2}$. In addition, a $\Lambda_{c}$ trigger (Recoil 4) was also instituted which required at least three charged tracks in the recoil chamber.

A thousand $6250 \mathrm{bpi}$ tapes were used to store the 25 million events recorded over the period of November 1980 through June 1981. The ratio of 137 to $170 \mathrm{GeV}$ data was about $2: 1$. Reconstruction of the raw events proceeded through two passes. The first pass involved the reconstruction of the forward charged tracks and their 3-momenta. In addition, charged and neutral track reconstruction for the recoil chamber was carried out. The second stage made use of the Čerenkov, calorimetry and muon wall data to identify the charged particles and reconstruct the neutral particles in the forward spectrometer.

Event reconstruction was a computationally intensive process. The Cyber 175 
computers at Fermilab required about $1 / 2$ second of CPU time per event for the first stage (Pass 1). Consequently, the Pass 1 reconstruction was accomplished by a set of widely dispersed computer systems: the Cyber 175's of Fermilab, an IBM $3033 \mathrm{~N}$ at the National Research Council laboratories in Ottawa, Canada and six IBM 370/168 emulator processors constructed at the University of Toronto. Pass 2 was somewhat less CPU intensive (requiring only 1/6 of a Cyber $175 \mathrm{CPU}$ second) and so was carried out on the Cyber 175's at Fermilab and on VAX 11/780 minicomputers at the University of California at Santa Barbara and the University of Colorado at Boulder.

As a final step, selected information from each reconstructed event was packed onto a set of data summary tapes (DST). In addition, preliminary analysis programs were run to select events with desired characteristics, that is, events most likely to contain charm particles (i.e., an identified kaon, proton, $\Lambda$, or $K_{s}^{0}$ ). 


\section{CHAPTER 2 \\ EXPERIMENTAL APPARATUS \\ and}

DATA RECONSTRUCTION

\section{(2-1) Outline}

We begin with a discussion of the accelerator itself. The basic components of the accelerator are outlined along with their modes of operation. Further details concerning the accelerator can be found in reference [114]. By following the path of the proton beam, we are lead to the Tagged Photon beam line wherein the creation and tagging of the photons is spelled out. Finally, we arrive at the bulk of this chapter in which the various elements of the Tagged Photon Spectrometer are discussed.

\section{(2-2) The Proton Accelerator Facility}

Fermilab is located on a 7000 acre site 35 miles west of Chicago, Illinois. Figure 2-1 provides a schematic layout of the facility. The acceleration of the protons follows a four stage process starting with the Pre-accelerator. Further acceleration follows in the Linac and the Booster up to an energy of $8 \mathrm{GeV}$ whereupon the proton beam is subsequently injected into the Main Ring for the final acceleration up to $400 \mathrm{GeV}$. After reaching this final energy, the beam is extracted over a 1 second interval and distributed into the three main experimental areas-Meson, Neutrino and Proton. The whole process of acceleration plus extraction is repeated every ten seconds. A more detailed exposition follows.

\section{(2-2-1) Pre-accelerator}

The proton beam begins as a stream of hydrogen gas from a pressurized cylinder. An ion source strips off the electrons and channels the resultant proton beam into 
a $30 \mathrm{~cm}$. long accelerating column within which the protons are accelerated up to an energy of $750 \mathrm{keV}$ by an electrostatic field produced by a Cockcroft-Walton generator. The Linac receives the resultant beam which consists of 15 pulses of protons per second (each pulse lasting $10 \mu \mathrm{sec}$ ). Each pulse consists of $1.25 \times 10^{13}$ protons. All the equipment, including the gas bottle, ion source, power supplies and instrumentation, are stored within a large metal dome maintained at a voltage of $750 \mathrm{kV}$.

\section{(2-2-2) Linac}

In contrast to the Pre-accelerator, the Linac makes use of time varying electric fields to accelerate the protons. The Linac itself consists of nine cylindrical cavities each with a diameter of one meter and a length of sixteen meters. The interior is evacuated (as are all the beam pipes) and contains a set of drift tubes equipped with focusing quadrupole magnets. The protons travel down the centerline of the cavities through the $2 \mathrm{~cm}$. aperture in the drift tubes.

Acceleration proceeds in the following manner. As the cavities are tuned to resonate at $201 \mathrm{MHz}$, a standing electric wave of this frequency is established within the Linac. During one-half of the cycle, the field will accelerate the protons. To prevent deceleration during the subsequent half cycle, the protons must be shielded from the electric field. The interior of the drift tubes provide this function. Note that the spacing and length of the drift tubes must increase in step with the ever increasing speed of the protons.

At the completion of the process, one obtains 15 pulses of protons per second at an energy of $200 \mathrm{MeV}$. Each pulse has a duration of $10 \mu \mathrm{sec}$ and consists of $6.0 \times 10^{12}$ protons. Note the current loss in the process. These unavoidable losses continue on through the whole multi-stage procedure. Both the Linac and Pre-accelerator are pulsed at a $15 \mathrm{~Hz}$ rate in order to meet the requirements of the Booster acceleration cycle. 


\section{(2-2-3) Booster}

Every $1 / 15$ of a second, the Booster drives a cluster of protons from $200 \mathrm{MeV}$ up to $8 \mathrm{GeV}$. This machine is made up of $9610-\mathrm{ft}$. long magnets arranged in a ring of 75 meters radius. Since this accelerator is an alternating gradient synchrotron, the Booster magnets have both focussing as well as bending capabilities. The magnets guide the protons through the circular path while the acceleration is provided by 18 distributed if cavities. In a $33 \mu$ sec interval, the protons accelerate from a speed of $\beta=0.57$ to $\beta=0.99$. Consequently, to constrain the beam in its circular path, the magnetic fields must increase from an initial value of $0.5 \mathrm{kG}$ to $6.7 \mathrm{kG}$. Furthermore, the driving if fields suffer a frequency increase from $30 \mathrm{MHz}$ to 53 $\mathrm{MHz}$. Each Booster pulse consists of up to $2.5 \times 10^{12}$ protons which implies that the Main Ring can receive up to $4 \times 10^{13}$ protons per second, but due to inevitable losses, the Main Ring actually holds only $2 \times 10^{13}$ protons for the ten second acceleration cycle.

\section{(2-2-4) Main Ring}

The Pre-accelerator, Linac and Booster are collectively known as the Injector to the Main Ring. The multi-stage process is necessary as each accelerator is limited both in the high and low energy end; hence one requires a set of machines that successively overlap in their energy ranges.

The Main Ring is a proton synchrotron comprising over 1000 magnets set into a ring of $1 \mathrm{~km}$. radius and buried six meters below ground level. It is divided into six equal size circular sections that are connected by six straight sections labeled A-0 through F-0. The magnet population is apportioned into $77420-\mathrm{ft}$. long dipole bending magnets, 192 7-ft. long quadrupole focussing magnets and 48 4-ft. long quadrupoles to adjust the beam in the straight sections. The magnets are arranged into cells of 8 bending (B) and 2 focussing (Q): QBBBBQBBBB; this pattern being repeated 84 times around the ring (except in the straight sections). In addition, there are other higher order magnets present for the fine tuning of the beam. 
After acceleration in the Booster, the protons are in the highly relativistic region; hence acceleration in the Main Ring will not significantly affect the speed of the protons. Consequently, the Main Ring operates at a fixed frequency of $53 \mathrm{MHz}$ (matching that of the Booster at the end of its acceleration cycle) with the result that the protons are distributed into buckets spaced $19 \mathrm{nsec}$ apart. Acceleration is provided by $18 \mathrm{rf}$ cavities housed in the straight section known as F-0. An energy increase of $2.8 \mathrm{MeV} /$ pass is provided implying that three full seconds are required for the acceleration from $8 \mathrm{GeV}$ to $400 \mathrm{GeV}$.

\section{(2-2-5) Beam Extraction and Utilization}

The final $400 \mathrm{GeV}$ beam of protons is extracted over a one second interval in the straight section A-0. The extraction is accomplished in two ways. In the first mode, one utilizes a resonance property of the accelerator. At equilibrium, the machine operates at a betatron frequency of $19.45 \mathrm{~Hz}$; that is, the protons traverses 19.45 oscillations about the stable equilibrium orbit for each circumnavigation of the Main Ring. However, there exist neighbouring destructive resonances at $19.33 \mathrm{~Hz}$, $19.50 \mathrm{~Hz}$, etc. To extract the beam, one adjusts the fields of the quadrupole magnets so as shift the betatron frequency to a unstable value. Eventually, the protons are shifted far enough from their stable orbits so as to enter the electrostatic field of the first extractor. Other magnets further downstream direct the spilled beam into the area known as the Switchyard.

The aforementioned method is used to shave off the proton beam over a one second interval. This is necessary as most experiments cannot handle the otherwise immense event rate of a short spill. In contrast, experiments utilizing neutrino beams require a maximum event rate. For these experimenters, a $1 \mathrm{msec}$ spill is produced at the end of the $1 \mathrm{sec}$ spill. In this case, pulsed magnets drive the required proton beam directly out of the Main Ring and on into the Switchyard.

The Switchyard is a large area north of the accelerator in which are located many bending and focussing magnets as well as electrostatic deflectors. This equip- 
ment is set up for the purpose of directing the extracted beam to the experimental areas. The typical procedure runs as follows:

1. The beam is refocussed into an elliptical configuration transverse to the beam direction.

2. The beam enters an electrostatic deflector in which one portion is in the positive region while the other is in the negative region; hence there will be different deflections for the two sections of the beam.

3. One portion encounters the field of a bending magnet and is given an even larger deflection while the other portion is simply deflected back onto the center line.

4. The deflected portion enters other magnets which carry the beam on into the appropriate experimental area. The undeflected beam cruises further ahead into the Switchyard where appropriate routing takes place.

The experimental stations are grouped into three main areas-Meson, Neutrino and Proton. The area known as Proton is itself divided into three stations-Proton West, Proton Center and Proton East. The tagged photon and broad band photon beam lines occupy Proton East. 


\section{(2-3) Tagged Photon Beam Line}

\section{(2-3-1) Electron Beam Line}

A schematic of the beam line is shown in figure 2-2. Protons are brought to bear upon a $30 \mathrm{~cm}$. long beryllium target (TEBY). A varied assortment of charged and neutral particles are produced in the subsequent reactions. The charged secondaries (and non-interacting protons) are swept out of the beam and into a beam dump by vertical dumping magnets. An exception is the muons produced at the target; they cannot be absorbed in the charged particle beam dump. As a result, these muons plus others produced from pion and kaon decays further downstream form a polluting cone of charged particles. A partial remedy is shown in the figurethe beam line is bent away from the original proton beam direction to minimize the muon flux in the experimental area. The residual muons are used for the calibration of some of the detectors within the experiment.

The remaining neutral beam is comprised of $\pi^{0 \prime} s, K_{L}^{0} s$ and neutrons. The $\pi^{0}$ component decays into two photons. These will subsequently convert into $e^{+} e^{-}$ pairs within the $0.32 \mathrm{~cm}$ thick lead converter located 12 meters downstream of TEBY. The other neutrals interact with the lead nuclei to produce a hadronic contaminant of charged particles.

Positively charged secondaries are swept out of the beam line while a collection of focussing and bending magnets (in conjunction with some collimators) steer and focus the negative electron beam onto a pair of vertical and horizontal collimators (CV423 and CH423 respectively). Just prior to the aforementioned, a horizontal bending magnet ( $\mathrm{BH} 415)$ provides the desired momentum dispersion in the electron beam. Adjustment of the current in this magnet will allow one to select the desired momentum while the opening size in the horizontal collimator ( $\mathrm{CH} 423)$ defines the momentum resolution $( \pm 2.5 \%)$.

Most of the $\pi^{-}$contamination is eliminated at this point in the beam line. At the lead converter, pions are created with much larger transverse momenta than 
the electrons (i.e., $300 \mathrm{MeV} / \mathrm{c}$ versus $10 \mathrm{MeV} / \mathrm{c}$ respectively). Consequently, most of the remaining pion contaminant is removed by the vertical collimator (CV423).

The momentum defined and purified electron beam is focussed and steered downstream until it reaches a copper radiator of 0.2 radiation lengths in thickness. Within this target, the electrons can interact electromagnetically with the copper nuclei via the bremsstrahlung process. Ideally, the interaction with a nucleus produces a recoiling electron plus a photon. The photon continues on into the liquid hydrogen target while the recoil electron is steered by a set of tagging magnets (AN440) into the tagging hodoscope array (see figure 2-3).

In reality, one has some complications occuring within the radiator. First of all, the radiator is thick enough so that the photon can actually convert to a $e^{+} e^{-}$pair. This is known as a trident event as we have the triplet: $e^{+} e^{-} e^{-}$. A set of counters A1-A10 exist in order to veto these events as well as electron-electron scattering cases. This vetoing was done during the subsequent data analysis as these anticounter events were simply latched during data-taking time (i.e., a bit was set in a latch module). A second major complication is that of double bremsstrahlung whereby the recoil electron can interact a second time within the radiator. The remedy for this is outlined in the next section.

This experiment used two electron beam energies-137 and $170 \mathrm{GeV}$. A plot of the electron yield (per proton) as a function of electron energy is shown in figure 2-5. The reconstructed photon energy spectrum for the $170 \mathrm{GeV}$ electron beam data is presented in figure 2-6.

\section{(2-3-2) Tagging System}

This system is comprised of (i) a lead glass-plastic scintillator hodoscope array, (ii) three tungsten-acrylic shower counters (C-counters) and (iii) the tagging system electronics.

Figure 2-4 is a schematic of the hodoscope system. The first component is a set of thirteen rectangular sheets of plastic scintillator (H1-H13). They are optically 
separated from each other and are individually attached to thirteen phototubes. Just behind the scintillators is a set of thirteen shower counters (L1-L13). Adjacent pairs of scintillators overlap slightly over the central section of each shower counter. Hence a valid tag requires the coincidence of an adjacent pair of scintillators $\left(H_{i} \bullet H_{i+1}\right)$ plus an appropriate energy deposit in the corresponding shower counter $\left(\left(H_{i} \bullet H_{i+1}\right) \bullet L_{i}\right)$.

The first two shower counters (L1-L2) are composed of 20 layers of $1 / 4^{\prime \prime}$ thick lucite with $1 / 4^{\prime \prime}$ thick sheets of lead sandwiched between pairs of lucite. The next six counters (L3-L8) are lead glass blocks (SF2) with dimensions: $2.5^{\prime \prime} \times 2.5^{\prime \prime} \times 23^{\prime \prime}$. The remaining blocks are also lead glass (of type SF5) and have the dimensions: $5^{\prime \prime} \times 5^{\prime \prime} \times 20^{\prime \prime}$. In addition, the counter L13 is placed with its long side facing the beam. These blocks have a larger cross section since they receive recoil electrons that have passed through the non-uniform fringe fields of the tagging magnets; hence they receive a greater dispersion than the other higher energy electrons. Being relatively thin, counter L13 also has a $1 / 2^{\prime \prime}$ inch thick lead sheet placed on its upstream face in order to ensure that the shower maximum occurs within the block. Each of the shower counters has a 10-stage RCA $6342 \mathrm{~A}$ photomultiplier tube attached to the downstream end of the blocks, except for L13, for which the phototube juts out to the side. The signal from the phototubes was delivered both to the tagging logic electronics and to LeCroy 2249 ADC modules (for signal integration).

It is known that lead glass will darken with time under continuous bombardment. However, this discolouration can be removed if the lead glass is periodically exposed to ultra-violet radiation. Since the counters L3 to L8 suffered the greatest flux of electrons (compared to L9-L13), they were collected together inside an aluminum box. The top of this box could be removed, and the blocks would be exposed to the ultra-violet radiation from a high intensity light source. This was usually done at weekly intervals over a 24 hour period. In fact, L1 and L2 suffered the greatest electron flux, but their lead-lucite construction does not suffer from 
radiation damage. The only penalty is a loss in energy resolution compared to the lead glass. It should be pointed out that $\mathrm{L} 1$ was actually used as a veto counter, as the flux here was so large that the bulk of experimental event triggers would have been due to low energy photons.

The light detected in the shower counters is actually due to Čerenkov radiation. The energy deposited gives a measurement of the energy of the recoil electron. Since the recoil angle is a function of momentum, a coincidence with the matching pair of scintillators provides one with a geometrically determined momentum measurement of the recoil electron. Note that this second measurement was only used to verify the validity of the first one. Denoting the electron beam energy by $E_{e}$, the recoil electron energy by $E_{e_{r}}$ and the photon energy by $E_{\gamma}$, the tagged photon energy can be expressed through the relation:

$$
E_{T A G}=E_{\gamma}=E_{e}-E_{e_{r}} .
$$

As noted previously, double bremsstrahlung events invalidate the above expression. Figure 2-7 is a schematic for the Tagged Photon Spectrometer. Three small shower counters were placed at the upstream side of the SLIC at beam line level. Each of these counters is a 20 radiation length thick tungsten-acrylic sandwich connected to a phototube. The central counter (C-counter) measures the energy of those photons that do not interact within the hydrogen target. In addition, the double bremsstrahlung photon could also convert into a $e^{+} e^{-}$pair within the target; the other two counters C-West and C-East would detect the pair event. Let $E_{C}$ denote the energy measured by the C-counters. The above relation is modified to read:

$$
E_{T A G}=E_{\gamma}=E_{e}-E_{e_{r}}-E_{C}
$$




\section{(2-4) Tagged Photon Spectrometer}

\section{(2-4-1) Hydrogen Target}

A schematic of the target flask may be found in figure 2-9. This is a double walled cylindrical flask $150 \mathrm{~cm}$. in length and $5^{\prime \prime}$ in diameter. The liquid hydrogen is contained within an inner flask of the same length and $2^{\prime \prime}$ in diameter. The wall of this container is constructed from a sheet of mylar 5 mils thick. Surrounding this container is another cylindrical sheet of mylar which also has a $1 / 2^{\prime \prime}$ layer of Rohacell foam attached to its inner surface. A vacuum exists between the two walls of the flask.

Hydrogen was chosen as the target material for several reasons. First of all, it is the simplest of atoms as it contains only one proton and with its low density ( $\rho=0.0708 \mathrm{~g} / \mathrm{cm}^{3}$ ), the recoil protons stand a good chance of escaping the confines of the target. The $2^{\prime \prime}$ of hydrogen represents $0.36 \mathrm{~g} / \mathrm{cm}^{2}$ of matter and the flask material presents $0.103 \mathrm{~g} / \mathrm{cm}^{2}$ to a recoil proton exiting radially from the target. The 1.5 meters of hydrogen maximizes the event rate while also minimizing the number of secondary interactions. This represents 0.17 of the radiation length and 0.25 of the nuclear collision length for liquid hydrogen.

\section{(2-4-2) Recoil Spectrometer}

Figure 2-10 is a schematic of this detector which was constructed at the University of Toronto. A detailed discussion of the recoil detector may be found in reference [54]. The exposition that follows will highlight the important aspects of the detector.

The recoil spectrometer is a two component machine. First one has three concentric cylindrical proportional wire chambers (PWCs). Surrounding them are four layers of segmented scintillation counters of which the inner two layers are plastic and the outer two contain liquid scintillator. The entire detector is concentric about the hydrogen target.

This device was designed for the express purpose of detecting the recoil proton 
from the reaction $\gamma p \rightarrow X p$. By 'detect', one means the following: (i) record the recoil proton trajectory, (ii) reconstruct the 4-vector of the recoil proton and (iii) identify the particle, that is, provide $(\pi, p)$ discrimination. This information is transmitted to the trigger processor and the data recording system. The trigger processor will select on-line those events with a high missing mass and a recoil proton. The missing mass $M_{X}$ is derived from the relation:

$$
\begin{aligned}
M_{X}^{2} & =2 k\left[\left(T^{2}-t\right)^{1 / 2} \cos \theta-T\right]+t, \\
& =2[k(p \cos \theta-T)-m T]
\end{aligned}
$$

where

$$
\begin{aligned}
k & =\text { photon energy }(40 \leq k \leq 160 \mathrm{GeV}), \\
t & =\text { Mandelstam momentum transfer variable }(\mathrm{GeV} / \mathrm{c})^{2}, \\
T & =\text { recoil proton kinetic energy }=-t / 2 m, \text { and } \\
\theta & =\text { recoil proton polar angle. }
\end{aligned}
$$

The recoil detector was expressly designed to facilitate the above tasks online by having the following characteristics:

1. The scintillators and PWCs produce fast signals available for processing.

2. The cylindrical geometry and segmentation of the scintillator permit multiparticle reconstruction and simplifies the corresponding calculations.

3. The low mass construction of the PWCs provides a good acceptance threshold in $t$ (see figure 2-11).

4. The energy resolution and $(\pi, p)$ discrimination is extended to large $|t|$ by use of a thick outer layer of liquid scintillator. At the same time, the first two layers of plastic scintillator are thin enough to minimize nuclear interactions by the recoil particles.

Some characteristics of the spectrometer follow:

1. The acceptance in azimuthal angle $\Phi$ is $331.5^{\circ}$. Polar angles $\theta$ are required to be greater than $20^{\circ}$. 
2. The momentum transfer $t$ can be computed for the range $0.06<|t|<1.4$ $(\mathrm{GeV} / \mathrm{c})^{2}$ with an energy resolution of $5-10 \%$.

3. Reliable $(\pi, p)$ discrimination is possible for $|t|<0.6(\mathrm{GeV} / \mathrm{c})^{2}$.

Particle reconstruction can be itemized into the following list:

1. The polar angle $\theta$ is determined from the PWC cathode wires as they form a set of wire hoops concentric about the beam line.

2. The azimuthal angle $\Phi$ is calculated from the set of hits recorded by the PWC anode wires that run parallel to the beam line. Note that this information is not required for triggering purposes.

3. Since other particles can be present, a cathode track must be matched with the corresponding scintillator sector through EET (end-to-end timing) in the Alayer (i.e., the innermost layer of plastic scintillator). The EET measurement is obtained by using two PMTs (photomultiplier tubes), one placed at the upstream end of the detector and the other at the downstream end. By using the downstream signal as a timing reference for the upstream signal, a time differential proportional to the position coordinate is obtained.

4. One can also time average the upstream and downstream signals with respect to the tagging signal to produce a TOF (time-of-flight) measurement. If the particle is of low enough energy to stop in the A-layer, then $(\pi, p)$ discrimination is possible purely on the basis of the TOF.

5. Otherwise, the particle will travel through at least two layers and hence there will be at least two energy deposit measurements available. This is sufficient to constrain the particle identification. The energy deposits are compared to predicted Bethe-Bloch $\mathrm{dE} / \mathrm{dx}$ energy losses for different particle identities. A subsequent least-squares fit allows one to postulate the most probable identity for the particle. 
(2-4-2a) Construction Aspects of the Recoil Detector

(2-4-2a(i)) Proportional Wire Chambers (PWCs)

At the anode planes, the three $\mathrm{PWCs}$ have radii of 18,36 , and $54 \mathrm{~cm}$. They have an active length of $200 \mathrm{~cm}$. and a radial thickness of $0.12 \mathrm{~g} / \mathrm{cm}^{2}$. The gap between the anode and cathode for each PWC is $6.35 \mathrm{~mm}$. A simplified, cutaway view of the PWCs is shown in figure 2-12.

Each PWC is made up of two concentric cylinders; each made of a low mass honey-comb structure (Rohacell) sandwiched between two sheets of mylar $8 \mu \mathrm{m}$ in thickness. Gold plated tungsten wires $20 \mu \mathrm{m}$ in diameter are stretched between two printed circuit boards attached to G-10 plastic hoops at each end of the PWC. The inter-wire spacing is $4 \mathrm{~mm}$. Lucite rings support the anode wires at three equidistant points along the length of the wires. The mylar sheet below the wires is coated with aluminum to form the non-readout cathode plane. The readout plane consists of 1,312 flattened copper wires glued to the inner mylar surface of the outer cylinder with a wire spacing of $1.524 \mathrm{~mm}$.

For readout, pairs of cathode wires constitute single channels. In the case of the anodes, each channel covers $2.5^{\circ}$ of azimuthal arc because the anode wires are read out in groups of 2,4 and 6 for the inner, middle and outer chambers respectively. Four meters of ribbon cables carry the signals out to the electronics rack which sits adjacent to the recoil chamber.

Typically, a particle track will produce one hit on an anode channel and a cluster of hits in the cathode channels. The electronics must identify the clusters, and calculate their centroids and widths. Processing proceeds in parallel for all chambers at an average rate of $300 \mathrm{~ns} /$ cluster. The resultant data is transmitted both to the trigger processor and the data logging system.

The chambers used a gas mixture of $81 \%$ argon, $15 \%$ isobutane and $4 \%$ methanol and were operated at voltages of $2.45 \mathrm{kV}$. Unfortunately, the inner chamber suffered breakdown problems. Sixteen spots on the cathode plane were identified as 
potential breakdown points by use of a radioactive source. Small oval mylar patches were glued onto these spots. By redefining the local electric field at these locales, the breakdown problem was solved (although the chamber voltage had to be kept 50 volts lower than the other two).

\section{(2-4-2a(ii)) Recoil Calorimeter}

The calorimeter is divided into fifteen azimuthally defined sectors-each sector covering $22.1^{\circ}$ of arc. In addition, each sector is comprised of four layers of scintillatorthe innermost two (A \& B) being plastic (NE114) and the outer two layers (C \& D) liquid (NE235A). In figure 2-10(a), note that the bottom $22.5^{\circ}$ of acceptance is lost in order to allow room for the PWC support trolley and signal cables. An additional $6^{\circ}$ is lost to the walls separating the sectors.

Each sector of the A \& B layers is constructed from sheets of NE114 plastic scintillator $5 \mathrm{~cm}$. in thickness and lengths of $240 \mathrm{~cm}$. for the B layer and $215 \mathrm{~cm}$. for the A layer. The A layer is shortened in order to allow for the installation of downstream PMTs (EET/TOF tubes). The radial edges of the sheets are tapered so that a better fit between adjacent sectors is created. Each sheet is wrapped with $8 \mu m$ thick aluminum foil for the dual purpose of creating a light-tight seal and for facilitating light transmission towards the PMTs.

The liquid scintillator is contained within three iron tanks, each spanning $110.5^{\circ}$ of arc. Furthermore, each tank is subdivided into ten sections- five each for the $\mathrm{C}$ \& D layers. Each compartment spans $22.1^{\circ}$ of arc with the $\mathrm{C}$ layer being $16 \mathrm{~cm}$. in radial thickness and the $D$ layer $10 \mathrm{~cm}$. in thickness. The sections are separated by $40 \mu \mathrm{m}$ thick aluminum walls. The ends of the tanks were sealed with $1.9 \mathrm{~cm}$. thick plexiglass sheets.

Since the scintillator would have corroded the metal walls of the tank, a protective layer of transparent epoxy paint was applied to all the inner surfaces. Total internal reflectivity for angles of incidence $<24^{\circ}$ was achieved by covering the interior surfaces with $3 \mu \mathrm{m}$ sheets of aluminized teflon with the index of refraction for 
teflon being 1.35 in contrast to 1.47 for the liquid scintillator.

A total of 220 PMTs were used for light detection. At the upstream end of the calorimeter, the calorimetry tubes were apportioned as follows: (i) three $2^{\prime \prime}$ PMTs per A counter, (ii) four $2^{\prime \prime}$ tubes per B counter, (iii) two 5" PMTs per C counter and (iv) three $3^{\prime \prime}$ tubes per D counter. For EET/TOF measurments, each A counter has two $2^{\prime \prime}$ fast timing PMTs-one each at the upstream and downstream ends of the detector. The output signals were processed by an array of TDC and ADC modules. A set of fast $(1 \mu s)$ 8-bit TDCs and ADCs processed the signals for subsequent transmission to the trigger processor. The same TDCs along with slower 12-bit ADCs (LeCroy 2285) transmitted the processed signals to the online data recording system. The voltages of the PMTs were controlled through a battery of programmable high voltage supplies (LeCroy LRS4032).

Phototube gains were monitored through the use of a nitrogen gas laser. The light was transmitted to the counters through a bundle of optical fibers optically coupled to the downstream end of the detector.

\section{(2-4-2b) Recoil Calibration}

Recoil protons from the data were used for the calibration of the detector.

For EET calibration, events were chosen in which (i) at least one PWC track was present, and (ii) the PWC track had to match up to a single active scintillator sector. A straight line fit was made to the equation:

$$
z_{A}=a+b * T D C
$$

where

$$
\begin{aligned}
z_{A} & =\text { the predicted position within the A layer, } \\
T D C & =\text { the EET-TDC measurement, and } \\
a, b & =\text { the fitted parameters. }
\end{aligned}
$$

TOF calibration required both EET and ADC calibration as a prerequisite. Events with the following characteristics were chosen: (i) ADC, EET-TDC and 
TOF-TDC information was available, (ii) cathode tracks had to be matched to a sector in the calorimeter, and (iii) the event had to be reconstructed as either $\pi$ or $p$ based on the ADC information. Once again, a straight line fit was made to the equation:

$$
T_{M}=a+\bar{b} * T D C
$$

The quantity $T_{M}$ is the time span predicted to occur between the tagging reference signal and the entry of the particle into layer $\mathrm{A}$. This requires a knowledge of the particle trajectory and its energy reconstruction. Note that $\bar{b}$ is a fixed parameter determined from the EET calibration- $a$ is the fitted parameter.

For the ADC calibration, it was found that the energy deposit $E_{d e p}(\mathrm{MeV})$ could be adequately related to the number of $\mathrm{ADC}$ counts $E_{A D C}$ through the following relation:

$$
E_{\text {dep }}=\frac{\left(E_{A D C}-E_{p e d}\right)}{a_{1}\left(1+a_{2} e^{-a_{3} z}\right)} .
$$

$E_{d e p}$ is predicted from Bethe-Bloch energy loss curves. $E_{p e d}$ is the number of pedestal counts from the $A D C$, that is, the number of counts when the input to the ADC channel is zero. The gain coefficients are $a_{1}, a_{2}$ and $a_{3}$. Finally, $z$ is the distance between the ionization point and the phototube cathode surface.

The form of this function agrees both with the results of an experimental cosmic ray study and a Monte Carlo simulation. In the actual calibration, one uses $N$ events and all fifteen sectors with four layers (ABCD) per sector. The overall minimization is done on the quantity:

$$
\begin{gathered}
\chi^{2}=\sum_{i=1}^{N} \sum_{j=1}^{4}\left(\frac{a_{1}^{j}\left(1+a_{2}^{j} e^{-a_{3}^{j} z_{i}^{j}}\right) E^{j}\left(T_{i}, \theta_{i}\right)}{\sigma_{i}^{j}}\right. \\
\left.-\frac{\left(E_{A D C}-E_{p e d}\right)}{\sigma_{i}^{j}}\right)^{2}
\end{gathered} .
$$

$E^{j}\left(T_{i}, \theta_{i}\right)$ is the energy predicted by the Bethe-Bloch $\mathrm{dE} / \mathrm{dx}$ energy loss relation with $T_{i}$ as the initial kinetic energy of the particle, and $\theta_{i}$ as the polar angle of the particle trajectory. $\sigma_{i}^{j}$ is an error estimate based on (i) the ADC pedestal width, 
(ii) photostatistical effects on the ADC width, (iii) the error in track path length due to uncertainty in counter thickness, and (iv) another error in path length due to the uncertainty in the polar angle $\theta_{i}$.

The minimization of the above function requires an iterative procedure as the quantities $T_{i}$ must be determined from some set of gain coefficients. Once the kinetic energies are determined, one can proceed to find the three gain coefficients for each of the sixty counters. These calibration constants were used in the subsequent offline data analysis. The trigger processor is not capable of handling all these numbers, so all fifteen counters in each layer used the same set of gain coefficients. Hence the trigger processor only required the use of twelve numbers for its computations.

With time, the PMTs suffered changes in their gain characteristics and so a periodic adjustment of their high voltages was necessary. The nitrogen laser system was used as the light source for the process. Reference [54] provides an account of this gain-matching program.

\section{(2-4-2c) Recoil Data Reconstruction}

Reconstruction implies the recreation of the event in terms useful for physics analysis. Specifically, one wishes to find the number of charged and neutral tracks present in the detector, and their corresponding 4-momenta. The latter implies the use of particle identification. Additionally, primary and secondary event vertices must be determined. To carry out this program, one collects and analyses the available data using combinatorial algorithms and statistical methods (i.e., leastsquares, maximum likelihood) in conjunction with the calibration parametrization of the detector to finally produce a reasonable event description. Of course, error analysis is always folded into the overall process. These not only include the usual $1 \sigma$ effects upon the various input, throughput and output parameters, but also the ambiguities inherent within the track candidate reconstruction algorithm. Leastsquares or maximum likelihood methods are vital to the eventual crystallization of the event. Often, it is necessary to place limits on the complexity of acceptable 
events. All these varied aspects of the reconstruction process are present for this detector system.

The E516 Spectrometer can be split into two parts defined by the event physics. The basic photon-proton process can be written as $\gamma+p \rightarrow R+X$. Here $R$ refers to the recoil proton or its fragmentation products, while $X$ represents the forwardgoing reaction products. The former are detected by the Recoil detector while the latter are reconstructed in the other detector systems.

Reconstruction of the recoil event begins with collection and checking of the various sets of available data-PWC cathode and anode hits, end-to-end timing (EET), time-of-flight (TOF), and ADC pulse heights from the calorimeter section. Pedestals are subtracted from pulse heights, threshold cuts are applied, EET counts are converted to $z$ positions, and necessary bookkeeping is carried out.

The first major reconstruction effort involves the recreation of the charged tracks present in the detector. The PWC cathode hit and EET data are utilized for this purpose. First, a search is made for the best tracks, that is, those that leave a cathode hit in each of the three chambers. One loops through the hits present in the inner and outer chambers. It should be pointed out that each of these hits is actually a cluster of hits as a given track will activate several cathode wires at a time. The width of this cluster provides the position error for the track. For each pair of inner and outer chamber hits, one creates an envelope defined by the upstream and downstream edges of the two chosen clusters. In the region where this envelope intersects the middle chamber, a search is made of the appropriate hits to see if one is present in this region. If so, a 3-point cathode track candidate is recorded and a $\chi^{2}$ calculated for this line. The resultant 3-point candidates are compared to the available EET position data in order to match the calorimeter data with the wire chamber results. For each track candidate, up to three EET matches are allowed.

It should be noted that the $\chi^{2}$ estimate was not determined by the usual 
method of a least-squares line fit. In order to shorten the processing time, the $\chi^{2}$ was estimated by using the deviation of the center of the middle chamber average cluster widths of all three chambers. Although a proper fit would have given a lower $\chi^{2}$, the number still produced a useful and reasonable result. This illustrates how computational restrictions sometimes require the use of approximations to minimize what is already a steadily increasing processing time.

To increase the track finding efficiency, a similar search is made of 2-point cathode tracks where, for some reason or other, the third hit was not recorded. In this stage of the algorithm, it is required that at least one of the hits must not already have been used in the 3-point track candidate list. In addition, an acceptable 2-point track must match up to an EET sector and a 3-point track must intersect the target near the corresponding 2-point track intersection. Finally, a search for kinked tracks is also performed. These are pairs of 2-point tracks (e.g., inner-middle and middle-outer) that match up to the same EET sector.

The next major stage involves the matchup of the cathode tracks with the calorimeter sectors and the subsequent 4-momentum reconstruction of the track. To reduce the ambiguity among the cathode track-EET matches, a search is made for 3-point and 2-point anode tracks with EET matches. The algorithm is similar to that used for the cathode tracks. In this case, both points in the 2-point tracks must not have been used previously in the 3-point analysis.

The cathode tracks were ordered by increasing $z$ position and each track was subjected to a particle identification and energy recontruction program. First, the track was assumed to be either a proton or pion that stopped in the innermost A-layer of the calorimeter. If in fact, only the A-layer registered any light, then the analysis would stop here. An appropriate kinetic energy would be reconstructed for the track. This involved the least-squares minimization of an expression involving the difference between the measured and expected energy deposits. The latter involved using the mass hypothesis, stopper/penetrator hypothesis, light saturation 
and attentuation corrections as well as the Bethe-Bloch $\mathrm{dE} / \mathrm{dx}$ calibrations. Minimization took place with respect to the optimum expected kinetic energy for that track. In addition, the A-layer stoppers made use of the TOF information (if available) for particle identification. This allowed one to label some stoppers as high energy electrons (delta rays) recoiling from the hydrogen target. The results were included in the overall $\chi^{2}$ of the track.

If more than just one layer in the sector recorded light, then the track was classified as a penetrator and the proton/pion hypothesis attempted in this case. If only the $A$ and $B$ layers were used, or an acceptable $\chi^{2}$ was determined, the analysis was terminated. Otherwise, the process was repeated but with the outermost lit layer deleted from the analysis. Once again, a $\chi^{2}$ cut or the presence of only the two innermost layers would terminate the process. In the end, the two outer $\mathrm{C}$ and $D$ layers were useful for improving the energy resolution of some tracks.

The results of this stage were subjected to a set of constraints which required that no track or sector could be used more than once, and as many tracks and sectors must be used as possible. Ambiguities were settled with the use of the kinematic reconstruction $\chi^{2}$ of the track-sector match. Unused sectors could be labeled as possible neutrals within the detector. A search for the most upstream hadronic track was made with a subsequent determination of the densest cluster of tracks that lay within the vertex window defined by the hadronic track's polar angle. Finally, the various geometrical and kinematic results were loaded into a FORTRAN common block for subsequent useage in physics analysis.

\section{(2-4-3) Analyzing Magnets}

Two magnets were used for bending the charged particle tracks in the $\mathbf{x z}$ plane of the spectrometer coordinate system. The first magnet, $M 1$, formed the low momentum arm in conjunction with the drift chambers D1 and D2. Magnet M2 was needed to bend the higher momentum tracks. Both magnets were run to produce an integrated field of five kilogauss-meters. Magnet M1 had one coil and so had to be run at 1800 
amps while M2, with two coils, only required 900 amps.

The vector field of the magnets was measured using the Fermilab 'Zip-Track' system. The details of this device and its use are spelled out in reference [48]. An absolute measurement of the fields was made using a NMR probe. The fields were parametrized by polynomials and the results used in the track reconstruction programs.

\section{(2-4-4) Drift Chambers}

As shown in figure 2-13, there are four drift chambers D1,D2,D3, and D4 which are used to record the trajectories of charged particles travelling through the forward spectrometer. Used in conjunction with the two analyzing magnets M1 and M2, the 3-momenta of the charged tracks can be reconstructed.

All the chambers share certain common features. They essentially consist of sets of field and sense wire planes sandwiched between planes of high voltage wires (see figure 2-14). The high voltage planes consist of wires stretched horizontally with a interwire gap of about $3 \mathrm{~mm}$. The exception is D4 where the plane is actually (i) a ground plane, and (ii) a sheet of aluminum bonded to a hexcell backing for rigidity. In the sense planes, one has field and sense wires alternating. Furthermore, these wires are set in three different orientations; the $\mathrm{X}$ plane has the wires stretched vertically while the $U$ and $V$ views are set $\pm 20.5^{\circ}$ with respect to the vertical. D1 has an additional $\mathrm{X}^{\prime}$ plane with the same orientation but offset horizontally with respect to the $\mathrm{X}$ plane.

Figure 2-15 illustrates the need for at least three different views if one wishes to measure the position coordinates of a track. Since D1 suffers from the highest rates, as it is so close to the hydrogen target, the $\mathrm{X}^{\prime}$ plane provides additional redundancy. D1 is made up of two assemblies of UVXX', D2 and D3 each have three assemblies of UXV, and D4 has only one assembly of UXV. Hence a charged track can have up to nine position measurements.

D1, D2 and D3 all have their sense wires set at ground and the field wires at a 
negative high voltage. The high voltage planes were set at typical voltages of about $2.3 \mathrm{kV}$ while the field wires in the sense planes were set to voltages between 1.7 and $1.8 \mathrm{kV}$. Chamber D4 had the sense wires operated at a positive high voltage and the field plane was grounded. The field wires in the sense plane could be set to small positive or negative voltages in order to establish an appropriate electric field configuration.

The sense wires were constructed of $25 \mu \mathrm{m}$ gold-plated tungsten wire and the field wires were $5 \mathrm{mil}$ beryllium-copper. For D1 through D4 respectively, the $\mathrm{X}$ plane cell sizes are $0.1875^{\prime \prime}, 0.375^{\prime \prime}, 0.625^{\prime \prime}$ and $1.250^{\prime \prime}$. The $\mathrm{U}$ and $\mathrm{V}$ plane cell sizes are smaller by a factor $\cos 20.5^{\circ}$. The chambers are filled with a 50-50 mixture of argon and ethane. In addition, D1 and D2 were found to suffer from breakdown problems due to the high rates experienced by these chambers. To remedy this problem, a small amount of ethyl alcohol vapor was added to the D1-D2 gas system for the express purpose of quenching disruptive ionizations.

How does a drift chamber work? Because of the high voltages set up in an appropriate gas mixture, a charged track will create an ionization trail along its path. If the field configuration is correct, the ionization electrons will drift at a constant speed towards a sense wire. Hence the position of the track will be linearly related to the drift time. This time of travel is measured in a somewhat odd manner. The signal that reaches the sense wire is processed to form a logic signal which is subsequently used as a START signal in a TDC module. A common STOP signal is generated from low level logic. By delaying the STOP signal, one buys the needed time for deciding whether or not to record the event (i.e., read out the TDC modules).

In the case of this experiment, the sense wires are connected in groups of sixteen to LeCroy DC201 amplifier-discriminator cards situated on the outside of the chambers. Twisted pair cables transmit the logic signals to a set of LeCroy 2770 TDC modules. The digitized numbers are read out and recorded onto magnetic 
tape.

\section{(2-4-4a) Drift Chamber Calibration}

There are basically five major steps in the calibration procedure: (i) calculate the gain coefficient of each drift cell, (ii) measure the relative timing between the cells that make up a single plane, (iii) establish an absolute timing reference, (iv) calculate the drift velocity for each plane, and (v) determine the relative spatial position of the drift chamber planes with respect to the spectrometer coordinate system.

The linear relation between the number of TDC counts and the time can be written as:

$$
t_{i}=t_{i}^{0}+g_{i} * t_{i}^{T D C},
$$

where subscript $i=$ the cell index,

$$
\begin{aligned}
t_{i} & =\text { time in nanoseconds }, \\
t_{i}^{T D C} & =\text { number of TDC counts }(0-255), \text { and } \\
g_{i} & =\text { the gain coefficient (ns/count). }
\end{aligned}
$$

By generating signals at fixed time intervals $\Delta t_{i}$, the gain coefficients can be calculated as

$$
g_{i}=\frac{\Delta t_{i}}{\Delta t_{i}^{T D C}} .
$$

If instead, one generates data at fixed times $t_{i}=t_{\text {constant }}$, then a set of relative time references can be calculated:

$$
t_{i R}^{0}=t_{\text {constant }}-g_{i} * t_{i}^{T D C} .
$$

An electronic pulser system was designed, built and operated for the express purpose of generating the calibration data used for determination of the aforementioned calibration constants. The details of the pulser construction and operation are spelled out in reference [47]. The system is controlled by the online monitoring software. To determine the TDC gains, a set of four different time delayed signals is transmitted to the DC201 cards, that is, counts of $60,120,180$, and 240 were 
used. The responses from the DC201s were used as the START signals-a common $S T O P$ was also provided. As outlined above, the differences in delay times were used to calculate the TDC gains. The process of cycling through the delay times and each assembly required about two hours of running time (with ten events per spill being recorded).

The data for determining the relative timing references was generated by low voltage $(\approx 5$ volts) pulses transmitted to the high voltage planes of each assembly. Output signals would be induced on the sense wires and subsequently used as the $S T A R T$ pulses. Once again, a common STOP pulse was provided. As the most uniform pulsing response was found to occur for large TDC values, the modules usually recorded times of about 220 counts.

With the relative timings now established, one can proceed to plot the overall time distribution for each plane. The gains and offsets of each TDC module is used to modify the centering and widths of the individual cell distributions. From the overall distribution, one can establish both the absolute timing reference and the drift velocity for the plane. The absolute timing reference is established by determining at what point the distribution starts rising. The drift velocity is calculated by dividing the cell size by the width of the time distribution.

Finally, the orientation of the drift chambers with respect to the overall spectrometer coordinate system must be established. A set of special data was recorded in which the regular beam was totally shut off by use of collimators and a trigger set up which required a coincidence between the upstream and downstream muon counters. (This is the 'cone of muons' mentioned previously in the electron beam section.) The analyzing magnets were turned off for these runs. The resultant straight line tracks are reconstructed in a straight forward manner. An examination of measured - fitted quantities allows one to make the correct position orientation.

In reality, various corrections must be made to the calibration constants. The reconstruction of tracks from actual photo-production data is used for this proce- 
dure. Plots of measured - fitted position coordinates allow one to unfold the required corrections. The calibration constants are stored in run indexed disk files for use in the offline reconstruction efforts. Finally, during the actual online data-taking runs, the TDC responses are compared to benchmark values stored on disk files. Significant deviations from the benchmarks resulted in a notice being submitted to the online personnel who could proceed to respond in the appropriate manner to the problem.

\section{(2-4-4b) Charged Track Reconstruction}

The algorithm is a multistage process, each stage consisting of the following steps: (1) looping through the various planes and assemblies in order to find matching sets of hits, (2) projecting these segments into other assemblies and/or chambers in order to find other matching segments, (3) performing line fits to these segments, and (4) selecting the best tracks from the set of candidates.

First, all TDC data is converted into position coordinates $(\mathrm{mm})$ for each of the $\mathrm{U}, \mathrm{X}$ and V planes. The first stage begins in D3. Each of three assemblies is cycled through in order to find matching sets of UXV triplets. The process involves searching for overlapping wires and then checking that the predicted $\mathrm{X}$ position (calculated from the $\mathrm{U} \& \mathrm{~V}$ data) corresponds with the recorded value of the $\mathrm{X}$ position. Upon completion, a search is then made for candidate track segments within D3. One proceeds from the best to worst case scenario.

(i) A three triplet search is made in which pairs of assemblies are looped through and a corresponding triplet in the third assembly is seeked. All three possible combinations of assembly doublet plus singlet are used.

(ii) The search is repeated, but in his case, only a doublet of hits is required in the assembly. For one reason or another, one of the planes has no recorded hits.

(iii) A final cycling of the chamber in which a plane in each of two assemblies has no recorded hits. This time, one triplet plus two doublets of hits are chosen. As can be seen, all the cases require a minimum of seven corresponding hits to 
be found out of the nine that are possible.

Next, the D3 track segments are projected back into D2. As with D3, a catalogue of D2 triplets has already been found. First the D3 segments are projected back into the target wherein the projected $Y$ position must be within $20 \mathrm{~cm}$. of the target centre. As the tracks are bent on the $X Z$ plane, no account of the magnetic field need be taken. Hence the D3 segment can be projected back into the first assembly of D2 in terms of the predicted Y position. A search is made of this assembly for corresponding triplets. When a correspondence is made, an $\mathrm{X}$ position check is made. Both the D3 and D2 segments are projected into the centre of the magnet M2. Here their predicted $\mathrm{X}$ coordinates can be checked for equality (within cuts). Furthermore, the bending angle in the $X Z$ plane can now be calculated and used in $\mathrm{M} 1$ (since the magnetic fields are almost equal) to project the D2 segment back into the target and a $X$ position cut made (again, $\pm 20 \mathrm{~cm}$.). Given a D3-D2 match, a search is carried out in the other six planes of D2 in order to find a minimum of three other corresponding hits for a total minimum requirement of six hits in D2. The entire process is repeated with the search beginning in each of the other two planes of D2.

The accepted D3-D2 segments are then projected back yet again into D1. First the $\mathrm{X}$ and $X^{\prime}$ planes in each of the two assemblies of D1 are cycled through in order to find corresponding $X X^{\prime}$ doublets. These are used first in the search for matching D1 segments. If no such doublets exists, then one reverts to individual plane searches. One starts with the downstream D1B assembly and projects back into the D1A assembly. The process is then repeated but with the initial search starting in D1A. Similarly, the D3-D2 segments are projected into D4 and a search of matching hits is made in the one assembly of this chamber.

With all D3-D2 segments (plus their extensions) finally catalogued, a filtering process is carried out in which only the best track candidates are kept. The choice depends upon the number of hits used, the $\chi^{2}$ of the track fit, the degree of matchup 
at the M2 centre, and the number of hits that a candidate shares with other tracks. This final list is stored for future use. So ends the first stage.

The second stage proceeds in a manner very similar to that of the first. In this case, the search begins in D2 with projections into D3, then D1 and finally D4. These D2-D3 candidates also undergo the quality control process before being stored for future use. One should note that care is taken to ensure there is no overlap with the already existent D3-D2 tracks. Both of these groups are sorted into various categories indicating which drift chambers the tracks passed through (i.e., D1-D2-D3-D4, D2-D3-D4, D1-D2-D3).

The third stage involves searching for those tracks that only passed through the first two chambers. Previously unused D2 segments (with at least seven hits) are projected back into D1 and filters applied to select only the most likely candidates. At this point, quality control is applied to all the tracks in order to flag the spurious ones. The key filter involved checking the number of hits that a track shared with other candidates. Too high a number resulted in that track being flagged as spurious. It was found that $99 \%$ of all D1-D2 tracks were actually spurious.

Another track search is carried out in the fourth stage. In this case, the unused segments of D3 are cycled through and projected into D4. The best of these are selected and stored. In the fifth stage, a search for a primary event vertex is carried out in which both the recoil and drift chamber tracks are used (excepting the D3-D4 tracks). This process involves using the recoil tracks to establish a vertex position estimate. The drift chamber tracks are subsequently added in to establish the final position. One requires the minimization of the sum of squares of the distance of closest approach (to the vertex). To accomplish the vertexing properly, an iterative, 5-parameter fit was made to all tracks (excepting again the D3-D4 tracks) in order to establish their trajectories and momenta (see reference [46]).

With this vertex established, a final search for D1-only tracks is performed. D1 being the most upstream chamber, it usually contains many hits and so a wide 
angle requirement was added to the algorithm so as to avoid the busy central region. Unused triplets of $\mathrm{D} 1 \mathrm{~B}$ were cycled through and projected into D1A. Besides the angle cut, the candidates had to point back at the target and a minimum total of five hits were demanded. The process would be repeated with the search starting in the D1A assembly. The best tracks were added to the final drift chamber track list.

\section{(2-4-4c) Primary and Secondary Vertex Reconstruction}

As a result of track reconstruction in the Recoil and Drift Chambers, one has information on the charged track 3 -momenta and positions. This is subsequently used to define the primary vertex for the event and to reconstruct the set of possible secondary vertices in which a neutral particle decays to two oppositely charged particles. A list of the chosen decays follows:

$$
\begin{gathered}
K_{s}^{0} \rightarrow \pi^{+}+\pi^{-} \\
\Lambda \rightarrow p+\pi^{-} \\
\bar{\Lambda} \rightarrow \bar{p}+\pi^{+} \\
\gamma \rightarrow e^{+}+e^{-}
\end{gathered}
$$

The first step is to find a primary vertex within the hydrogen target. One starts by using the intersection of the recoil tracks with the beam line to define the first approximation of the vertex coordinates. If no recoil tracks exist, then one must proceed to use the drift chamber tracks. Assuming that the recoil tracks exist, the number of vertices is reconstructed by making a cut on the weighted distance (in $z$ ) between successive tracks. Hence the tracks will be collected together into bundles, each of which represents one vertex. The primary vertex is defined by the most upstream bundle. Note that within each bundle, the vertex is defined by the most upstream recoil track.

One proceeds to add to the primary vertex bundle those drift chamber tracks that pass within a weighted distance of the recoil vertex, and have a subsidiary 
vertex defined by the drift chamber and upstream recoil tracks which lies within the hydrogen target. A least-squares analysis is applied to this enlarged bundle in order to find the optimum position. The algorithm involves the minimization of the weighted sums of squares of the respective distances of the recoil and drift chamber tracks from the primary vertex.

If the vertex passes a $\chi^{2} / d o f$ cut, then the analysis stops with no secondary vertex search. Otherwise, it is assumed that such vertices exist and all drift chamber tracks are cycled through in the subsequent search. Given a pair of tracks, they must meet certain specifications in order to be defined as neutral decay candidates:

(1) Neither track can be spurious and furthermore, both tracks must have passed through at least the first two drift chambers. Otherwise, no momenta would be defined for them. (This requirement is also used in the primary vertex reconstruction.)

(2) The pair must be oppositely charged.

(3) At least one of the tracks must not belong to the primary vertex. This is enforced through a cut on the weighted distance-of-closest-approach (DCA) between the track and the primary vertex.

(4) The DCA between the pair must be within $10 \mathrm{~cm}$.

(5) The neutral decay takes place before the centre of M1.

(6) The DCA between the neutral particle (whose momentum vector is defined as the vector sum of the track pair) and the primary vertex must be less than 10 $\mathrm{cm}$.

(7) The weighed distance between the primary and neutral vertices must exceed a specified amount. In other words, the secondary event must be distinct from that of the primary.

(8) Finally, no other recoil or drift chamber track can pass near the secondary vertex. Once again, the cut is enforced through a weighted DCA.

All secondary vertices must then be cycled through in order to ascertain their 
possible identities. First, pion masses are assumed for the decay tracks. If the invariant mass lies between 475 and $525 \mathrm{MeV} / c^{2}$, the neutral is flagged as a $K_{s}^{0}$ candidate. For the $\Lambda$ and $\bar{\Lambda}$ cases, kinematic analysis reveals that unless the neutral has a 3-momentum under $300 \mathrm{MeV} / \mathrm{c}$, then the proton will always have the larger momentum. Using this fact and the appropriate mass hypotheses, it is required that the invariant mass lie within the interval defined by 1100 and $1136 \mathrm{Mev} / c^{2}$. Finally, a converting photon is flagged if the invariant mass (assuming electron mass hypotheses) is less than $250 \mathrm{MeV} / c^{2}$.

\section{(2-4-5) Čerenkov Counters}

These provide the required particle identification of charged forward tracks in the spectrometer. Table 2-1 displays some key characteristics of the detectors. These detectors make use of the Cerenkov phenomenon-when a charged particle's speed exceeds that of light through a given medium, it is found that a cone of radiation is emitted at a polar angle $\theta_{c}$ with respect to the particle's line of flight. If the particle's speed is denoted by $\beta c(0<\beta<1 ; c=$ the speed of light in vacuum) and the refractive index of the medium is $n$, then one can write the following relation:

$$
\cos \theta_{c}=\frac{1}{\beta n} \text {. }
$$

From this equation, one can conclude that the threshold speed is

$$
\beta_{t h} c=\frac{c}{n}
$$

The E516 Cerenkov counters operate as threshold counters; that is, the particle identification is based on the fact that charged particles of different masses will have differing Cerenkov thresholds depending on their momenta and the refractive index of the medium. Discrimination is provided among $\left(e^{ \pm}, \mu^{ \pm}, \pi^{ \pm}, K^{ \pm}, p^{ \pm}\right)$, although the primary identification is for $(\pi, K, p)$.

Both counters are constructed from $1 / 4^{\prime \prime}$ aluminum sheets reinforced by aluminum I-beams for structural rigidity. However, the upstream half of $\mathrm{C} 1$ is con- 
structed from fiberglass as this portion of the detector is inserted within the magnetic field of M2. By using a non-conductive substance, one prevents the occurence of eddy currents in the walls of the counter. These currents could lead to the collapse of the counter walls if the magnetic field changed abruptly.

Each counter has its own access door and a set of circular portals encompassing each detector (see figure 2-16)-28 for $\mathrm{C} 1$ and 32 for $\mathrm{C} 2$. Each counter only used twenty of the available portals. These circular openings are where the Winston cones and associated PMTs are attached to the detectors. The ends of the detectors are sealed by 20 mil flexible, laminated sheets of aluminum and mylar.

The Čerenkov light is collected by a set of curved mirrors forming the primary mirror plane at the downstream end of each detector. These planes are split into 20 cells (see figure 2-17) which are further subdivided into four quadrants with 5 cells per quadrant. (The dotted lines indicate that cells $3 \& 5$ of each quadrant are actually made up of two mirrors each as single mirrors would have been too large and cumbersome to handle.) Each mirror is manipulated by seven dacron lines. The lines allow proper positioning of the mirrors without acting as high mass structures in the beam flux. As well, a $3 \mathrm{~cm}$. vertical gap exists between the upper and lower halfs of the mirror plane to prevent contamination from $\gamma \rightarrow e^{+} e^{-}$conversions in the hydrogen target.

In $\mathrm{C} 2$, the light is directly focussed into the Winston cones and PMTs. With the upstream end of $\mathrm{C} 1$ set inside $\mathrm{M} 2$, the PMTs had to kept at a safe distance from the fringe fields of M2. Thus the Winston cones and PMTs were set up to face upstream. Hence a second non-focussing mirror plane was set up to bounce the light into the phototubes. This setup is displayed in figure 2-18.

The mirrors are constructed from plexiglass sheets molded into the required curvature on large spherical aluminum molds. The finished sheet had a thin layer of aluminum applied to the concave surface. The resultant focal length is $6 \frac{1}{2}^{\prime}$.

The Winston cones are formed from epoxy spun onto a metal mandrel so that 
the resultant structure forms a cross section of a ellipsoid with the large end measuring $15^{\prime \prime}$ in diameter and the small end being $5^{\prime \prime}$ in diameter. Aluminum is evaporated onto the inner surface to make it reflective. Due to the focussing properties of the ellipsoid, any light entering the Winston cone will very likely be directed into the phototube attached to the small end of the cone ( $\left.5^{\prime \prime} \mathrm{RCA} 8854\right)$.

Anode signals from the PMTs were transmitted to LeCroy 2249 ADC modules for digitization and subsequent recording onto magnetic tape. The corresponding dynode signals were used in the E516 trigger logic.

\section{(2-4-5a) Črenkov Calibration}

1. Initially the refractive index of the chamber gases was monitored with the use of a gas chromatograph. As well, the presence of contaminants, especially $\mathrm{O}_{2}$, could be verified. However, the refractive index for $\mathrm{C} 2$ was particularly sensitive to the amount of $N_{2}$ present and it was found that the chromatograph was simply not accurate enough for the job at hand. So for this counter, the refractive index was monitored by measuring the onset of threshold for reconstructed $\pi^{ \pm}$tracks.

2. Mirror positions were surveyed by standard optical techniques. In addition, one required knowledge of the effective mirror boundaries as defined by the cone of light entering the phototubes. The data from a set of electron beam runs was used for this purpose. Originally, the runs were for the purpose of calibrating the SLIC detector. In these runs, a $5 \mathrm{GeV}$ electron beam was swept across the face of the mirror plane by a rotating magnet. The calibration procedure involved the comparison of reconstructed track positions with the number of photo-electrons detected in each of the Čerenkov cells.

3. Using incandescent and laser light sources, the mirror-Winston cone combinations were aligned for maximum efficiency (see reference [46]).

4. The ADC gains were determined by using light from a $N_{2}$ laser system (figure 2-19). Light is transmitted through a cluster of optical fibers with each fiber 
connected to one phototube. The intensity of the light can be controlled by the choice of an appropriate neutral density filter mounted on a filter wheel. If the correct filter is chosen, then a plot of the number of ADC counts will reveal a distribution with two prominent peaks-one being the ADC pedestal and the other being the single photo-electron peak. The gain is simply the difference between these two values. The laser system was also used to monitor the linearity of the ADC channels.

5. For reconstruction purposes, one needs to know the efficiency of each cell. This is the product of several effects: (i) multiple reflections off the mirrors and Winston cones, (ii) misalignment of mirrors and/or Winston cones, (iii) absorption of Cerenkov light by contaminants (i.e., $\mathrm{O}_{2}$ ) and (iv) the overall phototube efficiency. Using reconstructed track positions from photoproduction data, one can compare the observed number of photo-electrons with the predicted number. The ratio of these two numbers is the overall efficiency of each cell.

\section{(2-4-5b) Cerenkov Reconstruction and Particle Identification}

The eventual goal here is to provide some quantitative measure of a charged track's identity. This is given as a probability (properly normalized) of whether a track fits a mass hypothesis given the observed light pattern within the two Čerenkov counters.

The first step in the reconstruction process requires the conversion of the recorded $A D C$ counts into equivalent photo-electron counts making use of the appropriate calibration constants. Next, the predicted number of photo-electrons is calculated for each charged track that passes through one or more of the counters. As this involves integration over the path length of the track, the problem is converted into a discrete process whereby the trajectory is divided into a dozen segments in the first counter and ten in the second. Summation over the segments yields the predicted light levels for each of the five $(e, \mu, \pi, K, p)$ possible mass hy- 
potheses. It should be noted that the light emitted by tracks passing through the pair plane region of the downstream counter is excluded from the analysis as these photons were not detected by the counter.

To help in the identification of electrons and muons, information from the calorimetry analysis is utilized. First, the muon information (from the Hadrometer and Muon Wall reconstruction) is analysed. If the muon probability is greater than 0.75 , then the track is labeled as a muon. Otherwise, it is either flagged as a possible muon (and an appropriate weighting made), or it is processed as a possible electron (or positron). In the latter case, the track momentum as found by the SLIC and/or Outriggers is compared with the Drift Chamber results. Given a match, the track will be labelled as a possible electron.

Subsequent analysis uses the Cerenkov information. One loops through the tracks and Čerenkov cells in order to create sets of correlated tracks and cells. In these sets, it is possible for more than one track to emit light into the same cell, although in fact, $85 \%$ of the sets consisted of single track sets. Since particles of the lowest mass, namely electrons, create the widest cones of light, the algorithm cycles from the lowest to highest mass hypotheses in order to eliminate impossible configurations when the predicted light pattern is compared with the observed. Used in conjunction with the aforementioned calorimetry data, one can set minimum and maximum mass hypotheses for each track within each set.

Given the correlated sets of tracks, and given the possible mass configurations within each set, one can proceed to calculate a likelihood function for each configuration. This function takes the form

$$
\mathcal{L}=\frac{n_{\text {pred }} n_{\text {obs }}}{n_{\text {obs }} !} e^{-n_{\text {pred }}}
$$

That is, one is dealing with a Poisson distribution in which the probability of obtaining $n_{o b s}$ observed photo-electrons given a prediction of $n_{\text {pred }}$ photo-electrons. The predicted light level is intimately correlated with the assumed mass hypothesis. Some important qualifying remarks must be stated. First, it is the natural 
logarithm of the likelihood function that is actually used, and in order to decrease computational time, a lookup table is used rather than the actual functional expression. Furthermore, both the predicted and observed light levels are actually distributed in a Gaussian fashion; the smearing being the result of various effects such as mirror non-uniformities, phototube efficiency, and ADC output variability. Hence the simple Poisson expression is replaced by a double integration over the two Gaussians convolved with the original Poisson. The resultant expression retains a Poisson-like character.

The output of this stage supplies one with the probability of obtaining an observed light level given a selected mass hypothesis. One wishes to obtain the inverse of this: given the observed number of photo-electrons, what is the probability of obtaining a selected mass hypothesis. This is a textbook case of applying Baye's Theorem:

$$
P\left(A_{i} \mid B_{j}\right)=\frac{P\left(B_{j} \mid A_{i}\right) * \mathcal{P}\left(A_{i}\right)}{\sum_{i} P\left(B_{j} \mid A_{i}\right) * \mathcal{P}\left(A_{i}\right)} .
$$

$P(A \mid B)$ is the conditional probability (i.e., the probability of the occurrence of A given that $\mathrm{B}$ has already occurred) while $\mathcal{P}(A)$ is the apriori probability for the occurrence of $A$. The apriori probabilities for five mass hypotheses were initially assigned using the particle yield results of other experiments. An iterative procedure is executed in which the successive apriori's are calculated through an algebraic rearrangement of the above formula. The final results of this calculation are 0.12 , $0.08,0.65,0.11$, and 0.04 for the apriori expectation of obtaining electron, muon, pion, kaon and proton tracks respectively.

As a final step, the individual track probabilities (i.e., single track sets) were calculated for each of the five mass hypotheses and recorded onto the final set of data summary tapes.

\section{(2-4-6) SLIC}

SLIC is an acronym for Segmented Liquid Ionization Calorimeter [128]. Used 
in conjunction with the Outriggers, the detection, identification and 4-vector reconstruction of $e^{ \pm}, \pi^{0} s$ and photons that shower electromagnetically within the detectors can be accomplished. Similarly, when the SLIC is used in conjunction with the Hadrometer, one can reconstruct neutral hadrons (e.g., neutrons, $K_{L}^{0}$ ) that begin their hadronic showers within the confines of the SLIC.

Electromagnetic showers are the dramatic result of having the combined processes of bremsstrahlung and pair production produce a geometrically increasing cascade of electrons, positrons, and photons in a medium. A high energy electron will interact with a nucleus to produce a photon which subsequently converts to an $e^{+} e^{-}$pair. These leptons will in turn radiate photons which will pair produce and so on. The number of particles will exponentiate with increasing traversal of the material until enough energy has been expended to terminate the conversion and radiative processes. Traditionally, shower counters are comprised of interleaved layers of a dense material (e.g., lead) which initiates and develops the shower, and scintillator material (+ PMT) which samples the shower energy periodically.

The SLIC is essentially a box $16^{\prime}$ wide $\times 8^{\prime}$ high $\times 40^{\prime \prime}$ in depth. The electromagnetic (EM) showers develop in $1 / 16^{\prime \prime}$ sheets of lead that are separated by teflon coated corrugated aluminum sheets of 16 mil thickness. Lead being a scintillator poison, the lead sheets were sandwiched between 40 mil sheets of aluminum and the ends sealed with epoxy. Figure 2-20 is a schematic of the SLIC. The square wave corrugations, when filled with liquid scintillator, formed the counter channels. Each of these channels are $1 \frac{1}{4}^{\prime \prime}$ in width and $1 / 2^{\prime \prime}$ in depth. Towards the center of the detector, each channel counted as one counter while the outer channels are doubled up. The teflon was used to create the necessary condition for total internal reflection (i.e., angles of incidence greater than $70^{\circ}$ ).

As with the drift chambers, position determination is optimized by using three different orientations of the channels $(\mathrm{U}, \mathrm{V}$ and $\mathrm{Y})$. The $\mathrm{U} \& \mathrm{~V}$ counters are oriented as in the drift chambers. The $Y$ counters are oriented horizontally along the 
spectrometer $\mathbf{x}$-axis. In addition, these counters are split in the middle along the $\mathbf{y}$-axis by a doubly mirrored surface. There are 20 successive layers of the sequence UVY to form a total counter thickness of 20 radiation lengths. Both the $\mathrm{U} \& \mathrm{~V}$ views have 109 counters each apportioned into 38 single width counters and 71 double width counters. The $Y$ view is comprised of 116 counters of which 80 are single width and the remaining 36 are double width.

For any one orientation, the twenty successive channels are summed to form one counter, the light being summed within a sheet of BBQ-doped wavebar. This material absorbs the blue light emitted by the scintillator and re-emits the energy as green light. The light is detected by a single PMT (per counter) angled steeply with respect to the wavebar for maximum light collection efficiency. The single channel counters used a $2 "$ RCA 4900 model while the double width counters used 3" RCA 4902 phototubes. The anode signals were digitized by LeCroy Model 2285 ADCs for subsequent transferal onto magnetic tape. The corresponding signals from the dynodes were summed to form part of the hadronic trigger for the experiment (except for those $\mathrm{Y}$ counters in the pair plane).

\section{(2-4-6a) SLIC Calibration}

As for the recoil calorimeter and Čerenkov counters, a $N_{2}$ gas laser + fiber optic system was used to monitor the gains of the phototubes during the experimental run. An online monitor program compares the laser results with benchmark values and warned the online personnel if any results exceeded acceptable deviations. Furthermore, these numbers were written to tape for further offline analysis and for the creation of run number indexed calibration files.

The PMT gains were originally calibrated using the data created during a 5 $\mathrm{GeV}$ electron beam run. A gymballed magnet was used to sweep the beam across the face of the SLIC. Unfortunately, the results were somewhat unsatisfactory as there were some difficulties in tuning the beam energy. Furthermore, the SLIC phototubes, the 2285 ADC system and the laser pulser system were not completely 
understood at that time.

In lieu of the electron beam run, most of the calibration was carried out later using pair trigger data. This trigger consisted of the sum of the central six $Y$ counters in coincidence with either one of two paddle counters mounted at the edges of the SLIC. These counters extended in from the edge for a distance of two feet and were placed at beam line height. Reconstruction from the drift chamber data allows one to compute the energies of the $e^{+} e^{-}$tracks (which are the result of photon conversions within the hydrogen target). Hence the ADC pulse heights can be correlated with the actual energies of these tracks. The pair runs were performed about once per month.

The corner $U \& \mathrm{~V}$ counters, and most of the $\mathrm{Y}$ counters could not be calibrated using this data due to their placements. For these counters, one triggered on the background muon flux that streamed through the spectrometer. Originally, these runs were designed for hadrometer calibration. The muons are minimum ionizing and would deposit about $0.5 \mathrm{GeV}$ in the SLIC. This data was also used to produce a set of attenuation constants for each of the counters.

Further checks were made using the photoproduction data in order to fine tune the SLIC calibration. Isolated electron showers would be used as their energies could be obtained from drift chamber reconstruction and one could make use of the additional requirement that all three views (i.e., U, V, Y) must agree with one another. Another check on calibration was made by reconstructing photon pairs. The resultant $\pi^{0}$ mass peak provided an absolute energy scale for the detector.

In the end, the SLIC had a position resolution of $3 \mathrm{~mm}$. and an energy resolution of $\sigma_{E} / E=12 \% / \sqrt{E}$.

\section{(2-4-7) Outriggers}

These counters were essentially upstream extensions of the SLIC in that they increased the vertical acceptance for detection of electromagnetically showering particles. They were also known as the Outer Electromagnetic Counters, but as they 
were physically distinct from the SLIC and as they straddled the beam line, the term Outrigger became associated with them.

As can be seen in figure 2-21, the Outriggers consist of two aluminum-walled boxes $57 \frac{1}{2}^{\prime \prime}$ wide $\times 18 \frac{3}{4}^{\prime \prime}$ high $\times 11 \frac{1}{2}$ " deep. They lie in the space between D2 and M2 and are mounted on two posts bolted to the floor. The two Outriggers can be positioned vertically along the posts. The counters are also slightly skewed at an angle of $\pm 3 \frac{1}{2}^{\circ}$ with respect to the beam line. This matches the angle of the tracks at the edge of the Outriggers.

Each Outrigger consists of sixteen $1 / 4^{\prime \prime}$ lead plates (clad with 25 mil aluminum sheets) alternately interleaved with horizontally $(\mathrm{Y})$ and vertically $(\mathrm{X})$ oriented slabs of scintillator. Hence eight layers of lead+scintillator comprise each of the $X \& Y$ counters. There are a total of fifteen $Y$ and twenty-three $X$ counters per Outrigger. The $X$ counter slabs are $2 \frac{1}{2}^{\prime \prime}$ wide $\times \frac{1}{4}^{\prime \prime}$ thick while the $Y$ counter slabs are $1 \frac{1}{4}^{\prime \prime}$ wide $\times 1 \mathrm{~cm}$. thick. Overall, the Outrigger has a depth of 18 radiation lengths.

Light collection is different for each of the counter orientations. For the $\mathrm{Y}$ counters, the eight successive layers actually extend an additional $3^{\prime}$ beyond the vertical edges of the lead sheets. This additional material is bent appropriately so that the scintillator light is channeled into a lucite mixing block attached to a phototube (all PMTs for the Outriggers are $2^{\prime \prime}$ RCA Model 4900). The light from the $\mathrm{X}$ counter layers are channeled into BBQ-doped wavebars. The re-emitted green light is reflected off a mirror at the downstream end of the wavebar and up into a light guide connected to a phototube. Figure 2-22 displays this setup.

Note that as the Outriggers are within the fringe field of M2, all X phototubes were shielded with CO-NETIC+steel tubes. The CO-NETIC alloy has an especially high permeability while the steel has a high saturation point. The combination of the two materials (separated by an air gap) was found to provide adequate shielding from the M2 field [48]. It was sufficient to shield the $Y$ phototubes with a single 
layer of CO-NETIC.

A problem was noted (and corrected) for this setup of the $\mathrm{X}$ counters. It was found that the light from the 7 th and 8 th layers suffered relatively little frequency conversion within the wavebar and hence contributed an unusually high amount of energy to the PMTs. During the interim period between the 137 and $170 \mathrm{GeV}$ runs, these layers were masked resulting in a $25 \%$ decrease in the output from the seventh layer, and a $90 \%$ decrease from the eighth layer.

As with the SLIC, the PMT anode signals were digitized by the LeCroy Model $2285 \mathrm{ADC}$ system and written onto tape. The dynode signals were summed into a portion of the experimental hadronic trigger in addition to being used in a special $h i g h-p_{T}$ trigger.

Outrigger calibration was similar to that of the SLIC. Two $30 \mathrm{GeV}$ electron beam runs were made in which the Outriggers were lowered into the undeflected beam. The data from these runs established the counter gains. Furthermore, the data from the hadrometer muon runs was used to establish attenuation constants and relative gains for the counters. The overall energy resolution was $20 \% / \sqrt{E}$. The laser pulser system of the SLIC also monitored the PMTs of the Outriggers.

\section{(2-4-7a) Electromagnetic Calorimetry Reconstruction}

Both the SLIC and Outrigger used similar reconstruction algorithms. The differences will be noted as the discussion progresses. The goals of the programs were to (i) reconstruct photons $(\gamma)$, (ii) take pairs of photons and reconstruct $\pi^{0} s$ which decay almost exclusively to $\gamma \gamma$ pairs, and (iii) discriminate between leptons and hadrons by comparing the Drift Chamber momenta with those deduced from the calorimetry information. The algorithm had to deal with several major and minor problems. A major problem evolved from the fact that the wider hadronically induced showers tended to create satellite showers that could masquerade as separate particles. Muons left minimum ionizing $1 / 2 \mathrm{GeV}$ deposits that created a nuisance as (i) there were large variations in the actual energy deposit, (ii) most of the muons 
were not reconstructed by the Drift Chamber algorithms, and (iii) for the Outriggers, an extra $3^{\prime}$ of protruding scintillator in the Y-counters led to spurious pulses. As well, the detector resolution and calibration errors led to energy measurements that did not always match between the three $\mathrm{U}, \mathrm{V}$, and $\mathrm{Y}$ views.

The reconstruction process begins by the collating of the pulse height counts from the $2280 \mathrm{ADC}$ modules. Pedestals are subtracted and the resultant counts converted into energies $(\mathrm{GeV})$ by use of gain constants obtained from the calibration procedure. Each of the views are divided into cells, each cell being a contiguous group of counters that exceed an energy threshold. Furthermore, it is possible to subdivide a cell into sectors. A sector is a group of counters for which the central counter contains significantly more energy than its two nearest neighbours. Usually a sector corresponded to a single particle. An overall maximum of 80 sectors was allowed for both the SLIC and Outriggers. Some subsidiary cuts were also used in the formation of a sector. First, the central counter of a potential sector had to pass an energy threshold cut that was reduced if a Drift Chamber track landed within one counter width of the central one. If a track landed between two sectors, then that charged track was associated with both sectors. Finally, a sector with an energy deposit that was relatively small compared to the deposit of an adjacent sector would be eliminated from the candidate list as these sectors were assumed to be the satellite showers of a single large shower.

Once the sectors were established, the centre of gravity of each sector was calculated. This position was adjusted according to the results of a fit made to the known transverse shape of an electromagnetic shower. A stepwise regression fit was used to find which of the candidate sectors were significant and how to divide the total cell energy among the significant sectors. This technique will be discussed briefly below. Complete details concerning the procedure can be found in reference [58].

In the next step, the transverse energy distributions of the significant sectors 
were fitted to a linear combination of an electromagnetic and (wider) hadronic shower shape. During this fit, the weight of a counter could be increased if (i) it had more photo-electrons per unit energy, (ii) it had a small pedestal width, (iii) the detector had better shower statistics, (iv) a charged track landed within one counter width of the sector centre, or (v) the sector contained a relatively large energy deposit. Following the fit, the error for the sector energies was calculated. This quantity depended upon the shower and photo-electron statistics, whether the incident particle was thought to be one that produced electromagnetic or hadronic showers, and the total energy deposit.

Up to this point, the algorithm has been virtually identical for both the Outriggers and the SLIC. Now, the reconstruction process diverges for the two detectors simply because the SLIC represents a far more complex reconstruction problem at this stage. We begin with the Outriggers.

First, the charged track information is used to remove sectors that would otherwise be considered as photon candidates. Then all possible XY combinations are looped through in order to find that set of $\mathrm{XY}$ views which contains the minimum number of photons necessary to explain the presence of all sectors. In fact, one rarely found more than two photons in the Outriggers. Hence the combinatorics were never a problem.

The same could not be said for the SLIC. One begins with a candidate particle list made up of all UVY triplets wherein the predicted $U$ coordinate calculated from the $\mathrm{V}$ and $\mathrm{Y}$ coordinates matched up with the recorded $\mathrm{U}$ coordinate. If it happened that a charged track matched to all three views, then the required accuracy of the predicted $\mathrm{U}$ coordinate was reduced.

Stepwise regression, a method based on a least squares fit, was used to find the optimal set of particle candidates along with their fitted energies and energy errors. Essentially one is trying to relate a particular choice of candidate particle energies to the observed sector energies. By using the stepwise technique, one adds 
or deletes a candidate from the fit depending upon the effect of that candidate on the fit. In this way, only one correlation matrix needs to be calculated and the number of operations which must be performed on this matrix is minimized.

Once the process is complete, $\pi^{0}$ reconstruction begins. First, photons are identified as those particles that do not match up to charged tracks or to neutrals in the Hadrometer. Energy cuts allowed one to almost eliminate untracked muons and satellite showers. Valid photons were required to deposit at least $2 \mathrm{GeV}$ in the SLIC (or $1 \mathrm{GeV}$ in the Outriggers). All pairs of valid photons were collected and their corresponding invariant masses calculated. The deviation from the accepted value of the $\pi^{0}$ mass was parametrized through the relation:

$$
\chi^{2}=\left[\frac{\left(M_{\gamma \gamma}^{2}-M_{\pi^{0}}^{2}\right)}{\delta M^{2}}\right]^{2} .
$$

$\delta M^{2}$ is the error on the $\gamma \gamma$ invariant mass squared. It is dependent upon the photon energy errors and the opening angle between the two particles. The latter quantity is of consequence only for high energy $\pi^{0} s$. Further analysis (described in reference [48]) was carried out in order to create a probability parametrization of the $\pi^{0}$ candidates. Figure 2-23 is a mass plot of some $\pi^{0}$ candidates found in the SLIC.

\section{(2-4-8) Hadrometer}

Positioned just downstream of the SLIC, this detector records the hadronic showers created by neutral hadrons (i.e., neutrons, $K_{L}^{0}$ ). Hadronic showers are fundamentally different from the electromagnetic variety. The incident hadron will interact inelastically with a nucleus producing a host of secondary hadrons. These will proceed to interact inelastically with other nuclei producing ever increasing numbers of particles. Ultimately, only the ionizing component of the shower is actually detected and about $30 \%$ of the total energy is lost in the breakup of nuclei, nuclear excitation, and the nuclear emission of nucleons. The interaction scale is called the 
nuclear interaction length which is significantly larger than the material's corresponding radiation length. Hence the typical hadronic calorimeter is usually quite large.

The E516 Hadrometer is no exception to this rule. It is $16^{\prime}$ wide $\times 9^{\prime}$ high $\times$ $4^{\prime}$ in depth. Once again, this is an interleaved counter consisting of 36 layers, each of which is a $1^{\prime \prime}$ thick steel sheet followed by $3 / 8^{\prime \prime}$ of acrylic scintillator. The scintillator strips (each $5.7^{\prime \prime}$ wide) are collected together in groups of nine (depthwise) to form one counter. Figure 2-24 displays the outlay of the counters. As can be seen from the schematic, the Hadrometer is split into two modules each having one set of 33 $\mathrm{X}$ counters and two sets of $\mathrm{Y}$ counters (each set containing 19 counters). The light from the scintillator strips is channeled into 5" EMI $9791 \mathrm{~KB}$ phototubes.

The previously mentioned muon runs were used to establish attentuation constants and relative gains among the counters. Data from a $\pi^{-}$beam run was used to establish an absolute energy calibration for the detector. The muon runs required the coincidence of the upstream and downstream muon counters, and the Cerenkov system.

The resultant energy resolution was determined to be $75 \% / \sqrt{E}$. As a result, the Hadrometer's importance lay in its ability to identify rather than to provide precise reconstruction. As mentioned previously, this was the only detector capable of identifying the passage of neutral hadrons through the spectrometer. It could also supplement the drift chamber information with regard to the charged hadrons. Additionally, this detector could positively identify neutral hadrons that might otherwise have been reconstructed as photons by the SLIC. It's most important function was as a component of the E516 hadronic trigger [61]. It was also used to discriminate between hadrons and those muons depositing a minimum ionizing track.

\section{(2-4-8a) Hadrometer Reconstruction}

In order to find the neutral hadrons that shower in this detector, it is first necessary 
that those showers associated with charged tracks be accounted for. A search is made through all the individual counters as well as the sums of the corresponding upstream and downstream counters in order to find and catalogue those counters (or their sums) that contain significantly more energy than their neighbours.

Having compiled the significant counters, an attempt is made to match Drift Chamber tracks to the XY sets of significant counters. Given a successful match, the transverse energy distribution is fit to an average hadronic shower shape in order that this particle could be excluded in the subsequent neutral hadron search. The calculated energy was provided by the Drift Chamber information minus that energy deposited in the SLIC, and in addition, was constrained not to exceed the energy deposit found in the Hadrometer.

After subtracting out those counters associated with charged tracks, the search through the remaining significant counters was resumed. Corresponding $\mathrm{X}$ and $\mathrm{Y}$ views were matched together (taking into account attentuation along the length of a scintillator slab) and the neutral list compiled. Provision was made for a maximum of two neutral hadrons on each side (west and east) of the Hadrometer.

\section{(2-4-9) Muon Counters}

The upstream muon wall consisted of eight large scintillator counters, four on each side of the beam. These were located in slots between concrete shielding blocks situated between the tagging system and the hydrogen target. As a whole, these counters measured $5^{\prime}$ vertically and $6^{\prime}$ horizontally. The signals from the PMTs were discriminated and fed into the low level trigger logic. Their principal use was as coincidence counters in the muon run trigger.

The downstream muon wall follows the one meter of steel that immediately follows the Hadrometer. It is comprised of fifteen slabs of $1 / 2^{\prime \prime}$ thick acrylic scintillator. Figure 2-25 show the layout of the counters. Twelve are $10^{\prime}$ high by $18^{\prime \prime}$ wide. Of the remaining three, two measure $3 \frac{1}{2}^{\prime} \times 2^{\prime}$ and the central counter is $2^{\prime} \times 2^{\prime}$. Lucite light guides connect the scintillator sheets to $5^{\prime \prime}$ EMI $9791 \mathrm{~KB}$ phototubes. 
The steel of the Hadrometer plus that of the following wall is thick enough to stop all particles except for those muons above a $5 \mathrm{GeV} / \mathrm{c}$ momentum threshold. The resultant signals from these muons are (i) discriminated to set a latch bit, and (ii) used as a TDC START signal. The latter information can be analyzed offline to produce a vertical position for the muon track.

The downstream muon wall was used a component in the muon run trigger, and most importantly, as a key component in the dimuon trigger. The latter was used to find $J / \psi \rightarrow \mu^{+} \mu^{-}$decays [47].

\section{(2-4-9a) Muon Identification}

The results of this algorithm were provided in the form of a yes/no format. The identification process was developed through a study of $\psi \rightarrow \mu^{+} \mu^{-}$decays. There are sets of filters used depending on whether or not the tracks were reconstructed by the Drift Chambers. Most of the untracked muons originated in the primary proton target far upstream from the experiment. We start with the tracked muons.

Given a hit in one of the downstream muon counters, the $y$ position deduced from the TDC time must be consistent with the projected position of the track. Next, from 1 to $5.5 \mathrm{GeV}$ must be deposited in either of the $\mathrm{X}$ or $\mathrm{Y}$ views of the Hadrometer. The widths of these deposits must be no more than $12^{\prime \prime}$ in the $\mathrm{X}$ view and $10^{\prime \prime}$ in the $\mathrm{Y}$ view. Furthermore, if one calculates the ratio between the upstream energy deposit and the total deposition, then this ratio must lie between 0.16 and 0.87 in either of the $\mathrm{X}$ or $\mathrm{Y}$ views.

The requirements for the untracked muons are similar. First, the total energy deposition must be between 1.6 and $3.7 \mathrm{Gev}$. As well, at least two of the following three conditions must be met:

(1) The aforementioned ratio of the upstream to total energy deposition must be between 0.2 and 0.8 in the $\mathrm{X}$ view.

(2) Similarly for the $Y$ view. 
(3) The particle must be located at least one meter east of the Hadrometer midsection. It is in this region where the extraneous muon flux peaks.

\section{(2-4-10) Data Acquisition System}

This includes both the hardware and software required to gather, monitor and $\log$ the experimental data. All the hardware is controlled through the CAMAC system. This is a system to standardize the method of transmission of data and control signals between the instrumentation modules and some digital controller. As a corollary, CAMAC also defines the precise mechanical format of both the modules and the crate that contains them together with some signal and power supply conventions. The most common type of controller is a computer of some sort. E516 used a DEC PDP-11/55 minicomputer as the master controller over the data acquisition system.

CAMAC can be used in several configurations. This experiment used the branch configuration in which up to seven CAMAC crates can be linked together with the computer through a common link called the branch highway. A device known as the branch driver acts as the interface between the computer and the CAMAC system. There were three such branch highways in E516, each branch using a Jorway 411 branch driver. The individual crates can hold up to 23 modules. Furthermore, each crate must contain a crate controller module which acts as the interface between the computer I/O bus and the CAMAC dataway. (The dataway is the interconnecting highway located at the back of every crate used for transmitting data and control signals as well as for delivering power to the individual modules.)

\section{(2-4-10a) Low Level Trigger Logic}

NIM logic modules are used for the discrimination of the many signals from the spectrometer. Subsequently, the resultant logic signals are utilized in the first level of low level triggers used for event selection. A variety of triggers were employed but the major one was $T A G \bullet H$. 
There are three basic occurences possible when a photon enters the hydrogen target: (i) the photon proceeds through the target with no interaction taking place until it is detected within the $\mathrm{C}$-counter, (ii) the photon converts to an $e^{+} e^{-}$pair, or (iii) the photon undergoes a hadronic interaction with a proton. Of the three, the first is the most common, and the second has a cross section two hundred times greater than the third. A further complication involves the fact that the recorded hadronic data sample was to be enhanced with charmed events. This latter requirement was handled by the trigger processor (the high level trigger).

$T A G \bullet H$ was set up in order to maximize the hadronic sample while minimizing the pair rate. In the end, this trigger reduced the pair rate by $99.5 \%$ which implies that the data sample was a 50-50 split between pair and hadronic interactions. (Remember that the subsequent high level trigger will reduce this pair sample to a very low level.) This trigger can be written as the logical relation:

$$
T A G \bullet H=T A G \bullet((H+D i \mu) \bullet \bar{C}) .
$$

$D i \mu$ refers to the dimuon trigger which involves the coincidence between the second Cerenkov counter and the downstream muon wall (in addition to some scintillator paddle counters). This trigger was used to detect $J / \psi \rightarrow \mu^{+} \mu^{-}$decays. The full details of the trigger setup plus the analysis of the data collected with this trigger are spelled out in reference [47]. The symbol $C$ refers to the logic signal obtained from the C-counter.

In coincidence with a veto from the $\mathrm{C}$-counter, there must be a hadronic interaction $H$. This signal is based on the relation

$$
E_{h}>0.4 *\left(E_{\text {beam }}-E_{\text {tot }}\right)=0.4 * E_{\gamma} .
$$

$E_{h}$ is the amount of hadronic energy determined from the sum of signals from the Outriggers, SLIC and Hadrometer (with no signals taken from the SLIC pair plane). The energy from the Hadrometer is weighted by a factor of 5 in order to boost the 
detected hadronic energy for those events on the low energy tail of the Hadrometer resolution. Alternatively, one could say that with this weighting factor, the width of the measured $E_{h}$ distribution is minimized.

$$
E_{h}=E_{O E C}+E_{S L I C}+5 * E_{H a d}
$$

$E_{\text {beam }}$ is the electron beam energy (all energies are in units of $\mathrm{GeV}$ ), and $E_{\text {tot }}$ is the sum of the energies found in the tagging lead glass blocks. The threshold value of 0.4 was determined experimentally. The details are spelled out in reference [46].

Finally, $T A G$ is a complex coincidence among the following quantities:

$$
T A G=T \cdot \bar{A} \bullet \overline{E_{\text {tot }}^{\left(\begin{array}{c}
\text { too } \\
\text { igh }
\end{array}\right)}} \bullet \overline{S C R} \bullet B E A M \bullet \overline{\left(\begin{array}{c}
\text { computer } \\
\text { busy }
\end{array}\right)}
$$

$T$ is the fan-in of the tagging blocks. $\bar{A}$ is a coincidence between $\overline{L 1}$ (the first tagging counter veto), and the pre- and post-isolate vetoes. These last two veto any electrons from the prior and/or posterior beam buckets-recall that the $53 \mathrm{MHz}$ accelerator frequency results in particle 'buckets' spaced $19 \mathrm{~ns}$ apart. This measure ensures a more reasonable event rate. $E_{\text {tot }}^{\left(\begin{array}{c}\text { too } \\ \text { high }\end{array}\right)}$ is the upper limit on the fan-in of tagging pulse heights. It vetoes on those beam events with more than one electron in a bucket. $S C R$ refers to the radio-frequency noise emitted from the beam magnets' power supplies. $B E A M$ is the time interval known as the beam gate, and (computer $\left.\begin{array}{c}\text { cusy } \\ \text { bus }\end{array}\right)$ refers to that time interval when the PDP-11/55 is recording an event.

All event and calibration triggers were conveniently cataloged according to their corresponding bit pattern in a 16bit word. What follows is a listing of all of the E516 triggers preceded by the octal representation of the bit pattern.

1 The dimuon trigger otherwise known as USER-1.

4 The $h i g h-p_{T}$ trigger was also called USER-3. This required a coincidence between $T A G \bullet H$, energy from the Outriggers, and energy from the east or west sides of the SLIC. As its name suggests, the idea is to collect events with unusually high transverse momentum in the forward system. 
$40 T A G \bullet H$ was highly prescaled in order to enhance the charm sample collected by RECOIL- 1 through RECOIL-4 triggers.

100 The PAIR trigger is described in the SLIC calibration section.

200 The $\gamma$ trigger is the coincidence $T A G \bullet C$. As this was the most copious trigger, it was heavily prescaled.

400 RECOIL-1.

1000 RECOIL-2.

2000 RECOIL-3.

4000 RECOIL-4. (The RECOIL triggers will be discussed in the trigger processor section.)

10000 TRIG-13 is a spare trigger used in the Outrigger, Drift Chamber and Hadrometer muon calibration runs. In addition, it was used for the calibration runs involving the $e^{-}$and $\pi^{-}$beams.

40001 Trigger for upstream laser pulses used in the recoil calorimeter calibration and monitoring.

40004 Trigger for the downstream laser pulses transmitted to the Outriggers and the Hadrometer phototubes.

40020 The same downstream laser this time transmitting light pulses to the SLIC and Čerenkov counter PMTs.

40100 Trigger indicating that the pedestals of all ADCs have been sampled.

40400 The Drift Chamber pulser events are recorded when this trigger bit pattern is set. (Note that the ' 40000 ' series of calibration and monitoring events are all sampled between beam spills.)

(2-4-10b) Data Logging and Monitoring

As has been previously noted, computer control is through a PDP-11/55 computer. Along with $248 K$ of regular memory for user software, an additional $256 K$ of bulk memory was installed for buffering an event before it was finally written onto a 
6250bpi 9-track magnetic tape mounted on one of two STC tape drives. A userwritten program formatted the data before it was recorded on tape. The computer used the DEC RSX-11M V3.2 operating system, the Fermilab MULTI data acquisition software package plus user written software specific to the experiment.

Interfacing between the computer and the trigger logic was provided by a device known as the Bison Box. This generated a computer interrupt for data retrieval from the CAMAC crates whenever an event trigger was received. Furthermore, it created timing signals that defined the limits of the beam spill, and it transmitted

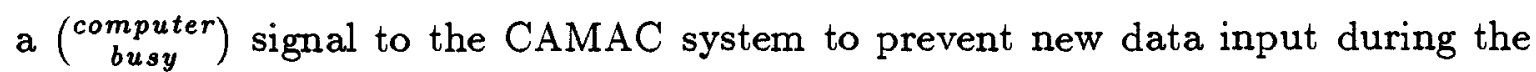
ongoing readout.

MULTI is a Fortran-based program written by J. F. Bartlett at Caltech and modified for Fermilab use. It carries out the basic functions of acquiring data from CAMAC-based hardware and writing it to tape, performing user defined analysis of the input data, and displaying the results in a graphic mode. As well, MULTI can monitor predefined quantities and compare them to benchmark values.

In E516, a separate monitor package (written by the experimenters) was used to prompt and control the laser pulser, Drift Chamber pulser, and pedestal events. Results were stored on tape and analyzed online for comparison with benchmark values. Deviations beyond $3 \sigma$ resulted in a message being transmitted (both by CRT and hardcopy) to the online personnel for their attention. Furthermore, the various scalers (e.g., number of tags) were monitored and written to tape. Finally, a host of high and low voltages were recorded using three 32-channel 12 bit LeCroy Model 2232 ADC modules. Again, these numbers were monitored and written to tape.

\section{(2-4-11) Trigger Processor}

The ECL-CAMAC Trigger Processor System is an example of the array of programmable, specialized, high speed digital systems that are quietly revolutionizing the methodology of experimental (and theoretical) particle physics. For the ex- 
perimentalists, the problem has always been one of enhancing the recorded data sample with events of the desired qualities. High speed NIM logic can reduce the input rate of $10^{6} / \mathrm{sec}$ down to $10^{4}$, but the computerized data logging systems are only capable of handling from 100 to 200 events per second. It is at this stage that one requires a high speed system capable of accomplishing the complex (and otherwise time consuming) computational tasks required for proper event triggering. The E516 Trigger Processor accomplishes the required tasks within a $10 \mu \mathrm{sec}$ interval. In contrast, the same task on a CDC Cyber mainframe would require about 0.2 seconds of $\mathrm{CPU}$ time.

The processor is an extension of the NIM logic philosophy of having a modular, high speed, and flexible system. The modules make extensive use of high speed ECL logic and are packed into modified CAMAC crates. The crates have ECL backplanes and a TTL-ECL converter module to allow for communication between the NIM and ECL logic. Hence diagnostics and system simulation can be carried out directly with the use of the online PDP11 computer. Programming is done by interconnecting the modules at the front panels (as in NIM) but here one uses both data and control lines. No master clock is used for the system. Rather the program path is controlled through $R E A D Y$ lines; each module begins processing when all its input $R E A D Y$ s are set, and sets the output $R E A D Y$ lines when the processing is finished. In this way, the high speed capabilities ( $\leq 50 \mathrm{~ns}$ ) of these modules is optimally exploited.

The key module is the MLU (Memory Look Up). Each of them consists of 4096 16bit high speed RAM memories. One precalculates functions of arbitrary complexity on the PDP11 using all possible values for the input variables, and the results are stored in a tabular form in the MLU memory. In this way, time consuming calculations can actually be done within $50 \mathrm{~ns}$ on a MLU unit.

Other modules include the following:

The Stack module is another memory unit comprising $3216 b i t$ RAM chips. It 
is used to store input data and intermediate results.

A double index DO-LOOP INDEXER used in the track finding algorithm.

A Track Finder module for generating tracks and the parameters associated with them.

A 3-fold General Logic Module capable of performing logical operations upon control signals: $((A \pm B) \pm C) \bullet G$.

A Quad $4 b i t$ function module acting as a $40 \mathrm{MHz}$ scaler for counting desired quantities such as the number of tracks in the recoil chamber.

A 3-fold FANOUT unit and the previously mentioned ECL to NIM level converter.

The basic E516 trigger involves selecting those events most likely to have diffractively produced charm particles. That is, the photon, in interacting with the target proton, converts to a $c \bar{c}$ pair and the recoil proton is ejected into the recoil chamber. By reconstructing the 4-momentum of the proton and photon, the forward mass (i.e., 'missing mass') of the charmed system can be calculated and triggered upon within the required $10 \mu s e c$ period.

Figure 2-26 displays the Trigger Processor algorithms in a simplified form. Step one requires the reconstruction of all possible charged tracks within the recoil spectrometer. As seen in figure 2-27, the PWC chambers have the indices (I,J,K) associated with them. The calorimeter ADC information is indexed by the sector number (1-15) and the scintillator layer (A,B,C,D). Denote the ADC information by $\left(\mathfrak{L}_{A}, \mathfrak{L}_{B}, \mathfrak{L}_{C}, \mathfrak{L}_{D}\right)$ and the PWC $z$ position data by $\left(z_{I}, z_{J}, z_{K}\right)$.

The data sets $\left\{z_{I}\right\}$ and $\left\{z_{K}\right\}$ (ordered in terms of increasing $z$ ) are fed into two stacks while $\left\{z_{J}\right\}$ is stored in terms of a hit array. Elements of this array are set to 0 or 1 depending on whether or not a hit was recorded in the appropriate $z$ interval. The DO-LOOP indexer loops through all I and $\mathrm{K}$ tracks and the projection onto the $\mathrm{J}$ chamber is calculated using the $\left(z_{I}, z_{K}\right)$ combination. Since the PWC chambers 
are equally spaced in the radial direction, the required relation is

$$
z_{J}^{p r o j}=\frac{\left(z_{I}+z_{K}\right)}{2}
$$

Comparison with the hit array elements allows a track match to be made. Given a track match, the projection to the target centre $\left(z_{v t x}\right)$ and the track's polar angle $(\theta)$ are calculated. Given the EET data, the projection into the A-layer can be calculated $\left(z_{A}\right)$ for matching with a PWC track. If no match is possible, then the track is classified as a NO MATCH. Given a track match, the ADC output from the appropriate sector can be processed for energy reconstruction and particle identification.

In terms of the processor hardware, the Stack modules are used to store the $\left\{z_{I}\right\}$ hits, the $\left\{z_{K}\right\}$ hits, and the output track parameters $z_{v t x}, \theta$, and $z_{A}$. The hit array for the $\left\{z_{J}\right\}$ data is a component of the Track Finder module which also computes $z_{J}^{\text {proj }}, z_{v t x}, \theta$, and $z_{A}$. The DO-LOOP indexer steps through all the $z_{I}$ and $z_{K}$ hits. A MLU unit is used to make the match between a PWC track and a calorimeter sector.

The calorimeter $\mathrm{ADC}$ information $\left(\mathfrak{£}_{A}, \mathfrak{E}_{B}, \mathfrak{L}_{C}, \mathfrak{E}_{D}\right)$ is input into MLUs in which the equivalent energy deposits $\left(E_{A}, E_{B}, E_{C}, E_{D}\right)$ are calculated. Along with the sector number, $z_{v t x}$ and $\theta$, these energies are input into another set of MLUs in which the 4-momentum reconstruction can take place assuming pion and proton hypotheses. Note that the TOF information is used in this section for possible particle identification. As well, note that the calorimeter data is also used in the reconstruction of possible neutral tracks (i.e., sectors for which there is no corresponding PWC track).

Final triggering decisions involve the calculation of the missing mass in addition to the comparison of several quantities. First of all, the most upstream vertex must be found that has only one positively identified proton track. This statement unfolds into the following: (i) based on $\chi^{2}$ cuts, the most upstream track is an acceptable proton, (ii) there are no neutrals, (iii) there are no more than two NO MATCH or 
backward tracks, and (iv) the total number of charged tracks does not exceed eight. Reconstruction of the photon energy allows for the calculation of the missing mass $M_{X}$. Used in coincidence with the above requirements plus that of $T A G \bullet H$, one forms the following two triggers:

RECOIL 2: $\quad 2.0 \leq M_{X} \leq 5.5 \mathrm{GeV} / c^{2}$,

RECOIL 3: $\quad 5.5 \leq M_{X} \leq 11.0 \mathrm{GeV} / c^{2}$.

The remaining triggers are somewhat more relaxed. RECOIL 1 asks that the missing mass be between 0 and $25 \mathrm{GeV} / \mathrm{c}^{2}$. It was given a low weighting (1/33) as this trigger would collect many events containing $\rho, \omega$ and $\phi$ particles whereas we wished to trigger predominantly on events containing charm. RECOIL 4 demands that at least three charged tracks exist at the most upstream vertex in the Recoil Chamber. It was believed that such events were more likely to contain the higher mass charm baryon states, particularly the $\Lambda_{c}^{+}$.

\section{(2-4-12) The 168 Emulator System}

A critical problem in modern experimental high energy physics has arisen due to the increasingly large amount of raw data recorded over the lifetime of today's experiments. It is simply that enormous amounts of computing resources are needed to process the raw data and output it into a form useful for analysis. This means that both great quantities of time and money are consumed before the physics analysis can proceed.

The 168 emulator system $(168 / \mathrm{E})$ is a response to this problem. It is a relatively cheap (compared to mainframe computers) computer system that can be run in a totally dedicated mode and hence is ideally suited to the task of running one set of data processing programs upon tens or even hundreds of thousands of events. The system constructed at the University of Toronto is based upon the original system built at SLAC for the LASS experiment $[64,65]$.

The emulators form a network of parallel processors that emulate the instruction set of an IBM 370/168 mainframe computer. All the peripheral aspects of a 
typical mainframe are absent so that data processing becomes the central task of these processors. Each processor unit consists of integer and floating point CPU chips in order to handle both single and double precision arithmetic. The integer CPU units are based on the 2901 family of bit slice arithmetic and logic chips while the floating point CPU was fabricated from discrete logic and read-only memory controlled sequencers. Each of the 168/E units represents $1 / 3$ to $1 / 2$ of the CPU capability of the $370 / 168$ mainframe. Each processor also contains $128 \mathrm{~K}$ bytes of data memory plus $96 \mathrm{~K}$ of program memory; the chips themselves being $55 \mathrm{~ns}$ static RAM.

In practice, the emulators were laid out as shown in figure 2-28. Four processors were set up in parallel between two busses. Each bus is connected to a large bulk memory unit-one for data and the other for programs. The program memory can hold up to $384 \mathrm{~K}$ bytes while the data bulk memory can store $512 \mathrm{~K}$ bytes. Through a UNIBUS, both of the processor busses are joined to a PDP 11/23 minicomputer. Finally, a high speed communications link exists between the PDP 11/23 and a VAX 11/780 minicomputer through which the raw data events and processed events can be exchanged.

Running a program on the emulators requires that the analysis package first be split into segments called overlays as the emulator memory is of limited capacity. Each overlay is compiled and debugged on a IBM mainframe with the resultant object modules then being translated into $168 / \mathrm{E}$ machine code. The final code is loaded onto the disk memory of the VAX before being downloaded into the program bulk memory unit.

Raw data events are initially stored on magnetic tape. The VAX transfers the data contained on one tape onto magnetic disk memory. Appropriately sized units of this data can be transfered to the data bulk memory. It should be noted that the data bulk memory must be apportioned among several types of data-program constants, raw data and processed events. 
Data transfers in the 168/E system can occur on any of three bidirectional ports collectively known as the Bermuda triangle. This name arose out of the early diagnostic tests of the system when data was known to mysteriously disappear in this network. Such problems no longer occured by the time the Toronto version was constructed.

The PDP $11 / 23$ acts as the overall controller of the system. It can send signals to the VAX asking it to initiate a raw data transfer from disk to the data bulk memory. Inversely, the PDP will also transfer the final processed events back to the VAX and onto disk memory. After the contents of one tape have been processed, the final results are transfered onto virgin tape. Additionally, the PDP handles the transfer of raw data, processed data and specific overlays between particular emulators and the bulk memory units. Finally, the diagnostic programs for the system are run on the PDP 11/23. These check the integrity of the each processor plus the bulk memory units as well as testing the Bermuda triangle circuits.

The essential features of the emulator system are summarized in Table 2-2. 


\section{CHAPTER 3 \\ DATA ANALYSIS \\ and \\ PHYSICS DISCUSSION}

\section{(3-1) Introduction}

Because of its large mass, the $\Lambda_{c}^{+}$has many weak interaction decay channels open to it. Consequently, the branching ratio for any specific mode is small and a signal can be easily overwhelmed by the combinatorial background. The three most prominent decay modes plus their branching ratios are the following: [1]

$$
\begin{array}{cc}
\Lambda_{c}^{+} \rightarrow p K^{-} \pi^{+} & (2.2 \pm 1.0) \% \\
\bar{K}^{\circ} p & (1.1 \pm 0.7) \% \\
\Lambda \pi^{+} & (0.6 \pm 0.5) \%
\end{array}
$$

The first channel offers the problem of proton and kaon identification as well as a large three-body combinatorial background. Furthermore, the $\Lambda_{c}$ mass is at the peak of the overall mass distribution and so the signal will be set against an overwhelming background.

The second channel has half the branching ratio of the first but it does have several advantages. First, it requires the identification of a neutral kaon which is copiously produced, and can be separately identified and selected. Hence one is effectively dealing with a pseudo two-body decay with reduced combinatorics. As well, the charmed baryon mass will occur in a section of the mass plot where the combinatorial background is considerably less than in the region where the phase space peaks. However, there remains the problem of proton identification as well as the following problem concerning the neutral kaon.

The neutral kaons exist in two states with different lifetimes and principal decay modes, namely the $K_{s}^{o}$ ( $K$-short) and $K_{L}^{o}$ ( $K$-long). Although both states are produced in a 50-50 ratio, the long lived state is difficult to detect as it usually 
decays far downstream of the production vertex. Hence, one normally searches for the $K_{s}^{o} \rightarrow \pi^{+} \pi^{-}$case. The procedure for detecting secondary vertices is stated in chapter 2 (section (2-4-4c)). Since one is dealing only with pions, minimal particle identification is required at this stage. Note also that one is in fact searching for the $\bar{K}_{s}^{0}$ which is indistinguishable from its counterpart as both decay to a $\pi^{+} \pi^{-}$ final state.

Reference [49] discusses the search for the decay $\Lambda_{c}^{+} \rightarrow \bar{K}_{s}^{0} p$. As in this thesis, the study in reference [49] was based upon the $170 \mathrm{GeV}$ data sample. However, the search for charm production was carried out assuming diffractive photoproduction of charm. That is, Recoil $2 \& 3$ triggers were required (the triggers are discussed in sections 2-4-10 and 2-4-11), and a clean recoiling proton had to be seen in the Recoil detector. This latter condition unfolds into the following: (i) one charged track in the Recoil detector positively identified as a proton, and (ii) no neutrals detected in the Recoil chamber. No discernible signal was seen. Instead, an upper limit for the cross section (times branching ratio) was calculated. Hence for the reaction $\gamma p \rightarrow \Lambda_{c}^{+} p X$ with the subsequent decays $\Lambda_{c}^{+} \rightarrow \bar{K}_{s}^{0} p$ and $\bar{K}_{s}^{0} \rightarrow \pi^{+} \pi^{-}$, an upper limit of $43 \mathrm{nb}(90 \% \mathrm{CL})$ was determined for the cross section times branching ratio.

The third channel seems at first very unfavourable with its low branching ratio. However, as with the second case, combinatorics are reduced by first searching for $\Lambda$ decays which are produced in fairly large numbers (although less so than the neutral kaons). Note that the remaining particle is a charged pion. Hence, minimal particle identification is required and so a distinct advantage can be seen over the second decay mode. The $\Lambda$ itself decays into two principal two-body channels:

$$
\begin{array}{rr}
\Lambda \rightarrow p \pi^{-} & (64.2 \pm 0.5) \% \\
n \pi^{o} & (35.8 \pm 0.5) \%
\end{array}
$$

The second channel requires the identification of both a neutral baryon and a neutral pion. This would result in a lower efficiency for finding this channel as 
both particles would have to be reconstructed from the calorimetry data. In any case, it has a significantly smaller branching ratio. Hence the first channel is most commonly used. Although the first case requires the identification of a proton, one can make use of the kinematics of this decay to increase the detection efficiency. In Appendix $\mathrm{A}$ it is shown that for momenta greater than $0.3 \mathrm{GeV} / \mathrm{c}$ (in the laboratory frame of reference), the proton momentum will always be greater than that of the pion. This makes for a good first approximation in proton identification. However, there are many background conditions that can produce a particle pair that has the invariant mass of a $\Lambda$. In the next section, a variety of information will be used to eliminate as many of those possibilities as possible.

Searches for charmed baryons were made in various decay channels: $\left(p, K, \pi,\left(\pi^{+} \pi^{-}\right)\right),\left(K_{s}, p,\left(\pi^{+} \pi^{-}\right)\right)$, and $\left(\Lambda, \pi,\left(\pi^{+} \pi^{-}\right)\right)$. A signal was observed only in the $\left(\Lambda, \pi^{+}\right)$channel. Given this signal, an attempt was made to find $\Sigma_{c}^{++} \rightarrow$ $\Lambda_{c}^{+}+\pi^{+}$. No discernible signal was seen.

\section{(3-2) The $\Lambda$ Filter}

A preliminary search for a charmed baryon signal found no evidence whatsoever of any signal in any of the channels listed above. The results indicated that a very careful and thorough approach would be required so as to minimize the combinatorial background. This idea was applied in the development of a $\Lambda$ filter. Many variables were examined to determine the sensitivity of the signal to imposed cuts, and from this large set of cuts, a simplified set was compiled for use in the $\Lambda_{c}^{+}$ search. This final set is discussed in this section.

Figure 3-1 displays the final $\Lambda$ signal output by the filters imposed upon the raw distribution. (Unless explicitly stated, the term $\Lambda$ will refer to both the baryon and its anti-particle.) A key variable controlling the ratio of signal to background and the width of the signal is the track category; that is, the number of drift chambers that the track traversed. The number of chambers traversed was coded as a bit set in a 4-bit variable. Hence, a track traversing the first two chambers is a 
category 3 track, a three chamber track is category 7, and a track traversing all four drift chambers is category 15 . For momentum reconstruction, a track had to travel through at least the first two chambers thereby implying that it was deflected out of the spectrometer by the field of the first magnet. These tracks had the poorest momentum resolution as reflected in figure 3-2(a) which shows the $\Lambda$ signal when both decay tracks are of this category. These will be classified as poor tracks. Conversely, tracks traversing either three or four drift chambers display the best momentum resolution and will be referred to as good tracks. The decrease in signal width and background is illustrated in figure 3-2(b) which shows the $\Lambda$ signal when only good tracks are used.

In choosing a filter, the idea is to produce the maximum reduction of background and minimum loss of signal. One is trying to use the maximum amount of information possible to identify and subsequently eliminate background processes that simulate a $\Lambda$ decay. A basic requirement for any decay is that the appropriate tracks must originate from some common vertex. The initial cut used for the secondary vertices requires that the distance-of-closest-approach (DCA) in real space for the track pair be no more than $10 \mathrm{~cm}$. This turns out to be far too generous and allows many unrelated tracks to be included in the $\Lambda$ sample. An optimum cut was found to be

$$
\begin{array}{ll}
\text { poor pion: } & D C A(p, \pi)<1.5 \mathrm{~cm} . \\
\text { good pion: } & D C A(p, \pi)<0.8 \mathrm{~cm} .
\end{array}
$$

Figures 3-12(a) and 3-12(b) display the accepted signal and rejected background respectively. A $59 \%$ reduction in background is affected by this filter - the best of the entire set. Furthermore, there is no discernible signal loss as shown in figure 3-12(b). A sketch of the above DCA distributions (and the cuts used) is displayed in figure 3-3(a) and 3-3(b).

In the original reconstruction, the track (correctly charged) with the larger momentum was identified as the proton. This is not sufficient for a unique proton identification: the Čerenkov identification probabilities (PROB) can provide further 
verification. These cuts are displayed in figures 3-4(a) and 3-4(b) and listed below:

$$
\begin{array}{ll}
\text { poor proton: } & P R O B(p) \geq 0.03 \\
\text { good proton: } & P R O B(p) \geq 0.05
\end{array}
$$

Figures 3-12(c) and 3-12(d) display the effects of this cut. One notes that there is a small $9 \%$ signal loss in the background distribution. Considering the background reduction of $48 \%$, the loss is more than acceptable.

Track quality is reflected in the number of drift chamber hits used in the reconstruction of the track. This is output as the number of degrees-of-freedom (DOF) on the data summary tapes (less the five parameters used in the track fit). Considerable background reduction can be acheived, especially for poor tracks. In this case, it was found that cuts on both tracks were required. Furthermore, the good tracks were split into category 7 and 15 subsets. The cuts are listed below and displayed in figures 3-5 and 3-6.

$$
\begin{array}{cc}
\text { category } 3 \text { pion: } & D O F(\pi)>6 \\
\text { category } 7 \text { pion: } & D O F(\pi)>13 \\
\text { category } 15 \text { pion: } & D O F(\pi)>14 \\
\text { category } 3 \text { proton: } & D O F(p)>6 \\
\text { category } 7 \text { proton: } & D O F(p)>14 \\
\text { category } 15 \text { proton: } & D O F(p)>15
\end{array}
$$

These DOF cuts as well as all others to follow individually represent small rejections of the background. The effect of all these remaining cuts is shown in figures 3-12(e) and 3-12(f). Collectively, these filters reject $47 \%$ of the background remaining after the proton probability cut.

Low momentum tracks were found to contribute some background. Hence the following momentum $(\mathrm{P})$ conditions were imposed:

$$
\begin{array}{cl}
\text { poor pion: } & P(\pi) \geq 0.6 \mathrm{GeV} / \mathrm{c} \\
\text { poor proton: } & P(p) \geq 2.0 \mathrm{GeV} / \mathrm{c} \\
\text { good proton: } & P(p) \geq 2.5 \mathrm{GeV} / \mathrm{c}
\end{array}
$$


Figures 3-7(a),(b), and (c) display the cuts.

Some background was found for those candidates whose distance-of-closestapproach to the main vertex was too large. The cut is shown in figure 3-8 and is listed below:

$$
\text { poor proton: } \quad D C A(\Lambda, v t x)<6.0 \mathrm{~cm} \text {. }
$$

It is possible that the $\Lambda$ is actually a $K_{s}^{o} \rightarrow \pi^{+} \pi^{-}$decay. Figure 3-9(a) shows these candidates when the pion mass is substituted in place of the proton mass. To minimize this contribution, a two part cut was imposed. First, the Cerenkov probability of the proton being a pion $(P R O B(p=\pi))$ had to exceed a value of 0.5. Second, the mass of the $\left(\pi^{+}, \pi^{-}\right)$pair had to lie within the $K_{s}^{0}$ peak. The following condition was used to reject this contamination.

$$
\begin{aligned}
\text { good proton: } & P R O B(p=\pi) \geq 0.5 \\
\text { and } & 484<M A S S\left(\pi^{+}, \pi^{-}\right)<512 \mathrm{MeV} / c^{2}
\end{aligned}
$$

Figures 3-9(b) and (c) display the $\Lambda$ mass distributions for those candidates rejected and accepted by the cut respectively. Of the original overall signal, $2 \%$ is lost with this cut.

One finds some background contributions from those candidates that decay either too close or too far from the main vertex. This is reflected in the following cut:

$$
\text { poor proton and pion: } \quad 0.3<D K L I F E<5.0
$$

The number DKLIFE is the ratio between the measured decay length and mean decay length expected for a $\Lambda$ of that momentum. The latter is given by the formula:

$$
\mathcal{D}_{\text {mean }}=\frac{P * c \tau_{0}}{m}
$$

where

$$
\begin{aligned}
\mathrm{P} & =\text { momentum }(\mathrm{GeV} / \mathrm{c}) \\
\mathrm{m} & =\text { particle mass }\left(\mathrm{GeV} / \mathrm{c}^{2}\right) \\
\mathrm{c} & =\text { speed of light }(\mathrm{cm} / \mathrm{sec}), \text { and } \\
\tau_{0} & =\text { mean particle lifetime }(\mathrm{sec})
\end{aligned}
$$


For the $\Lambda$, the proper decay length is $c \tau_{0}=7.89 \mathrm{~cm}$. Figure $3-10$ displays the distribution of this ratio and the imposed cuts.

The downstream calorimetry detectors provide information that can be used to label some charged tracks as electrons. This information is output as a probability flag. Some background was removed using this quantity. The filter itself is

$$
\begin{aligned}
\text { poor proton: } & \operatorname{PROB}(p=e)>0.01 \\
\text { poor pion: } & \operatorname{PROB}(\pi=e)>0.01
\end{aligned}
$$

As well, some of the $\Lambda$ candidates were also listed as $\gamma \rightarrow e^{+} e^{-}$conversions. No $\Lambda$ signal was found in this small sample and hence they were all eliminated from the final data set.

Finally, there is the question of track duplication. That is, there is a small but not insignificant subset of the data (4\%) in which two or more $\Lambda$ candidates can be found which share a track between them. This problem is much more prevalent in the original raw sample and so most of these cases have been eliminated by the other cuts. Since it is a small subset, one way of dealing with the problem is to simply quote what percentage of the signal suffers from this problem. The other is to find some way of choosing the so-called best candidate. Here again, as with all the other filters, one has to check for any bias created by the selection procedure. This procedure runs as follows:

One creates the product of the two Cerenkov probabilities for the proton and pion tracks.

The maximal value for each such event corresponds to the best $\Lambda$ candidate. All others are considered as background.

Strictly speaking, this filter is not an unbiassed selector of true $\Lambda$ events. However, as seen in figures 3-11(a) through 3-11(c), it does select more background than signal and hence represents a valid cut with minimal bias. In quantitative terms, relative to this particular subset of the data, $40 \%$ of the signal is lost, but with $70 \%$ background rejection. 
The event count after each of the $\Lambda$ cuts is shown in Table 3-1. The final sample of $\Lambda$ events for the $170 \mathrm{GeV}$ data sample is displayed in figures 3-13(a) through 313(c). As noted previously, the width of the mass distribution varies according to the track category. Hence, correct fitting of the distribution to a Gaussian signal plus linear background required that the sample be split into the three subsets created by splitting the tracks into good and poor categories.

Table 3-2 displays the results of the fit. In total, there are $(1.71 \pm 0.02) \times 10^{4}$ $\Lambda$ and $(1.10 \pm 0.02) \times 10^{4} \bar{\Lambda}$ particles in the $170 \mathrm{GeV}$ sample. Note that these numbers are not corrected for the host of efficiencies and acceptances inherent in the spectrometer system.

\section{(3-3) The $\Lambda_{c}$ Search}

A careful and systematic search was carried out for the $\left(\Lambda, \pi^{+}\right)$and $\left(\bar{\Lambda}, \pi^{-}\right)$decays of the $\Lambda_{c}$ charmed baryon.

Figures 3-19(a) and 3-19(d) display the final results of this search. In the $\left(\Lambda, \pi^{+}\right)$channel, a signal is seen with $51 \pm 14$ events centered at a mass of $2270 \pm 6$ $\mathrm{MeV} / \mathrm{c}^{2}$, a full width at half maximum of $36 \pm 11 \mathrm{MeV} / \mathrm{c}^{2}$ and a significance of $3.8 \sigma$.

As with the $\Lambda$ sample, the mass plots were fitted to a smooth polynomial background plus a Gaussian signal using the Maximum Likelihood Method [96]. A Poisson distribution is assumed for the likelihood function. Minimization of the negative natural logarithm of this function produces the optimum parameters which characterize the signal, namely, the mean mass, width, and size of the signal (as well as the parameters that describe the background). The minimization procedure is carried out by the MINUIT software package described in reference [119]. MINUIT is a well-tested and popular package used throughout the high energy physics community. In addition to providing the optimum estimates of the parameters, MINUIT includes algorithms for determining the errors on those parameters. These algorithms are based on well known statistical techniques described in references 
[96] and [119].

The significance is defined as the estimated signal size divided by the uncertainty in the signal population. By using a properly normalized Gaussian function, the peak intensity parameter multiplying the Gaussian also becomes the number of events constituting the signal. The error in this number is provided by the MINUIT program and includes the uncertainties in both the signal and background populations. Using the results of the fit, the significance is then $50.9 / 13.5=3.8$.

For cuts involving the variable parameters, i.e., the decay distance and the transverse momentum of the $\left(\Lambda, \pi^{+}\right)$, the signal, background and significance were determined for a wide range of parameter values in order to check that all three quantities (signal, background and significance) varied smoothly and appropriately as the cuts were changed.

The quoted error on the $\Lambda_{c}^{+}$mass is purely statistical and represents a $0.3 \%$ uncertainty in the mass. Previous studies [48] have shown that the systematic errors on our masses should be small. In reference [48], it is noted that the reconstructed mass for for $1900 K_{s}^{0} \rightarrow \pi^{+} \pi^{-}$events is $497.85 \pm 0.29 \mathrm{MeV} / \mathrm{c}^{2}$. The world average [1] is $497.72 \pm 0.07 \mathrm{MeV} / \mathrm{c}^{2}$. Hence the E516 value deviates from the world average by $0.03 \%$. Similarly in this thesis, the mass of the $\Lambda$ particle has been measured (see Table 3-2). The world average [1] for the mass of the $\Lambda$ is $1115.60 \pm 0.05 \mathrm{MeV} / \mathrm{c}^{2}$. From Table 3-2, the poorest value is $1116.0 \pm 0.6 \mathrm{MeV} / \mathrm{c}^{2}$, a deviation of $0.04 \%$ from the world average. The best value is $1115.66 \pm 0.04 \mathrm{MeV} / \mathrm{c}^{2}$ with a deviation of $0.005 \%$. Hence the systematic error on the measured $\Lambda_{c}^{+}$mass is believed to be negligible relative to the statistical error.

Returning to the mass plots of figures $3-19$, no corresponding signal in the anti-channel is seen as indicated in figure 3-19(d). Since the charmed baryon decays through the weak interaction, the natural width would appear as a delta function on the mass scale (10 MeV/c $/ \mathrm{c}^{2}$ per bin) used in the plots of figure 3-19. Hence the quoted width is a measure of the experimental resolution. A Monte Carlo simulation 
(see section 3-5 for a description of the Monte Carlo program) of the observed $\Lambda_{c}^{+}$ decay produced a width of $42 \mathrm{MeV} / \mathrm{c}^{2}$ - a value that is consistent with the observed width within the quoted uncertainty.

To check the background distribution, the $\left(\Lambda, \pi^{-}\right)$and $\left(\bar{\Lambda}, \pi^{+}\right)$distributions were plotted (using the same cuts as for $\left(\Lambda, \pi^{+}\right)$and $\left(\bar{\Lambda}, \pi^{-}\right)$) in figures 3-19(b) and 3-19(c). The first is the wrong charge case for a $\Lambda_{c}^{+}$state while the latter is disallowed by baryon number conservation. The absence of a signal in these latter plots shows that the mass peak of figure 3-19(a) is not due to some kinematic and/or acceptance effect and hence are further evidence that there is a real signal in the $\left(\Lambda, \pi^{+}\right)$case.

If the $\Lambda_{c}^{+}$mean mass and width from figure 3-19(a) is used in figures 3-19(b) through $(d)$ to measure the amount of signal in these cases, one obtains the following results: (i) $11 \pm 6$ events for the $\left(\Lambda, \pi^{-}\right)$channel, (ii) $-2 \pm 5$ events in $\left(\bar{\Lambda}, \pi^{+}\right)$, and (iii) $1 \pm 4$ events in the $\left(\bar{\Lambda}, \pi^{-}\right)$channel. The latter two are consistent with a null signal result while the first would only have a statistical significance of $\approx 1 \sigma$ and hence is also consistent with a null result.

To take into account the possibility of more than one combination per event, each $(\Lambda, \pi)$ combination in figures 3-19 was weighted according to the number of such cases in that event. It was found that such combinatorial contamination was, in fact, minimal $(<6 \%$ ), and so the final results were not changed by any significant amount. Table 3-3 displays the number of $\left(\Lambda, \pi^{+}\right)$combinations after each of the cuts to be discussed below. The final number of $\left(\Lambda, \pi^{+}\right)$combinations in figure 3-19(a) is 951 for an event count of 894 .

Another possible source for the signal in this decay channel is the strong decay of the strange particle $\Sigma(2250) \rightarrow \Lambda \pi$ with a reported mass between 2210 and 2280 $\mathrm{MeV} / \mathrm{c}^{2}$. However its published width is in the range of 60 to $150 \mathrm{MeV} / \mathrm{c}^{2}$ which is far larger than the $36 \mathrm{MeV} / \mathrm{c}^{2}$ width of the above $\Lambda_{c}^{+}$signal. It is also not a well established state [1]. 
Another possibility is that the $\Lambda_{c}^{+}$signal is a reflection from some other decay. That is, one or more of the decay particles has been misidentified and in fact the signal comes from the decay of some other meson or baryon. Mass plots were made in which the particle identities were cycled through pion, kaon and proton identities. No signal was discernible in any of the alternate mass hypotheses. We now proceed to discuss the cuts used in the analysis to filter out the background, and which led to the data sample displayed in figure 3-19(a).

\section{(3-3-1) Distance of $\Lambda_{c}^{+}$from Main Vertex}

Since the $\Lambda_{c}$ charmed baryon has a small lifetime $\left(\left(2.3_{-0.5}^{+0.8}\right) \times 10^{-13} \mathrm{sec}[1]\right)$, it is expected that it will decay to the $(\Lambda, \pi)$ state only a short distance downstream of the production vertex. Even a very high energy $\Lambda_{c}^{+}$with a lab momentum of 100 $\mathrm{GeV} / \mathrm{c}$ would have a mean decay length of only $3 \mathrm{~mm}$ in the laboratory frame of reference. Hence a cut on the decay distance is a powerful filter for discriminating between strange and charm decays. By demanding that the radial distance (in 3 -space) between the decay and main vertices be no more than $2 \mathrm{~cm}$, an optimal signal was achieved. Figure 3-14 displays the decay distance distribution and the cut imposed. Figure 3-18(b) is a mass plot of $\left(\Lambda, \pi^{+}\right)$after imposition of the cut. No demand was made that the $\left(\Lambda, \pi^{+}\right)$vertex be downstream of the main vertex as the experimental resolution did not allow for such a cut. When such a requirement was made, it was found that the signal was split into approximately two halves.

(3-3-2) Cut on the $\Lambda$ Mass

In addition, one minimized the background from the $\Lambda$ sample by cutting out the wings of the mass distribution. Specifically, one demanded that the $\Lambda$ mass be within the interval $1115.6 \pm 6.0 \mathrm{MeV} / \mathrm{c}^{2}$. This cut keeps $96 \%$ of the $\Lambda$ signal. The resultant mass distribution is shown in figure 3-18(c).

\section{(3-3-3) Well-identified Recoil Proton}

This experiment was outfitted with a recoil detector whose express purpose was to identify events with a single recoiling proton. The presence of this device was a 
unique feature of this experiment as no other photoproduction experiment has had a similar detection system with which to analyze the target fragments. The offline analysis of the charged recoil tracks (see section (2-4-2c)) produces, as one of its results, an identification flag for each of the reconstructed tracks. The values and interpretations of this flag, called MFLAG, are displayed in Table 3-4. Only the MFLAG $=4$ cases have been unambiguously identified as protons.

In that subset of the data where one (and only one) well-identified proton track was emitted from the main production vertex into the recoil detector, no $\Lambda_{c}^{+}$signal was observed. Such events are classified as diffractive recoil proton events. Figure 3-15 displays the rejected events while figure 3-18(d) displays the accepted events. A fit to the rejected sample indicates the presence of $-1 \pm 3$ events in the $\Lambda_{c}^{+}$mass region (2.22-2.32 GeV/c $\left.\mathrm{c}^{2}\right)$; a result consistent with no signal being present in this sample. This is direct evidence that the $\Lambda_{c}^{+}$charmed baryon is being created by a non-diffractive mechanism.

To illustrate, a diffracive mechanism of the type $\gamma p \rightarrow\left(\Lambda_{c}^{+} \bar{\Lambda}_{c}^{-}\right) p$ would require the observation of both a $\bar{\Lambda}_{c}^{-}$signal and the recoiling proton. Neither the $\bar{\Lambda}_{c}^{-}$nor the recoil proton are observed in association with the observed $\Lambda_{c}^{+}$signal.

Further studies of the charmed baryon signal and its relation to other variables related to the recoil chamber (e.g., charged and neutral multiplicities, total neutral energy deposition, polar and azimuthal angle of charged tracks) did not reveal any other significant correlations. Nevertheless, this does not diminish the significance of the primary observation as the recoil detector was designed specifically to detect diffractive events with a single recoiling proton.

\section{(3-3-4) Transverse Momentum of the $\Lambda_{c}^{+}$}

A non-diffractive production mechanism implies higher momentum transfers than those of diffractive collisions and hence the reaction products can have higher transverse momenta [115]. A cut on the transverse momentum of the $\left(\Lambda, \pi^{+}\right)$combination was found to significantly decrease background without affecting the signal. Specif- 
ically, it was required that $P_{T}>0.4 \mathrm{GeV} / \mathrm{c}$ be enforced. It is to be noted that the typical $\left\langle P_{T}\right\rangle$ of charmed mesons in photoproduction has been found to be about 1 $\mathrm{GeV} / \mathrm{c}$ [131]. Figure 3-17 displays those events rejected by this filter. The accepted events are displayed in figure $3-18(\mathrm{e})$.

\section{(3-3-5) One $\Lambda$ per Event and Well-defined Beam Energy}

Finally, a small reduction in background was possible by demanding a well defined photon beam energy and asking for only one $\Lambda$ per event. (In the latter case, one demanded the anti-particle for the anti-channel.) Figure 3-18(f) (and 3-19(a)) is a display of the final $\left(\Lambda, \pi^{+}\right)$mass distribution.

\section{(3-3-6) Previous Observations of the Decay Mode $\Lambda_{c}^{+} \rightarrow \Lambda \pi^{+}$}

A survey of all previous observations for the decay $\Lambda_{c}^{+} \rightarrow \Lambda \pi^{+}$(see Table 1-2) indicates a collective world sample of 19 events.

The first eight of these was observed by Baltay et al. [9] in an experiment carried out at Fermilab. A broad band neutrino beam was incident upon a $15 \mathrm{ft}$ bubble chamber filled with a liquid neon-helium mixture. Eight decays of the $\Sigma_{c}^{++}$ were reconstructed with the heavy charmed baryon decaying to $\Lambda_{c}^{+} \pi^{+}$. The $\Lambda_{c}^{+}$ subsequently decays to $\Lambda \pi^{+}$(with $\Lambda \rightarrow p \pi^{-}$). The $\Sigma_{c}^{++}$was seen by plotting the mass difference $m\left(\Lambda_{c}^{+} \pi^{+}\right)-m\left(\Lambda_{c}^{+}\right)$. A peak at $168 \pm 3 \mathrm{MeV} / \mathrm{c}^{2}$ was observed. The mass of the $\Lambda_{c}^{+}$was measured as $2257 \pm 10 \mathrm{MeV} / \mathrm{c}^{2}$.

In a similar experiment, Kitagaki et al. [16] observed $9( \pm 5)$ events of the decay mode $\Lambda_{c}^{+} \rightarrow \Lambda \pi^{+}$. Combined with the $10( \pm 6)$ events seen for $\Lambda_{c}^{+} \rightarrow \bar{K}_{s}^{0} p$, the average mass of the charmed baryon was calculated to be $2275 \pm 10 \mathrm{MeV} / \mathrm{c}^{2}$.

A photoproduction experiment (Forino et al. [20]), using the Omega prime spectrometer, was first to completely reconstruct an associated production event: $\gamma p \rightarrow \Lambda_{c}^{+} \bar{D}^{0}$. A tagged photon beam with energies between 20 and $70 \mathrm{GeV}$ was incident upon an emulsion target. The charmed baryon decayed to $\Lambda \pi^{+}$and the charmed meson decayed to $K^{+} \pi^{-} \pi^{-} \pi^{+}$. The $\Lambda_{c}^{+}$mass was measured as $2330 \pm 50$ $\mathrm{MeV} / \mathrm{c}^{2}$. 
Finally, a group using the same spectrometer (Adamovich et al. [34]) as Forino et al. [20] reconstructed three events, in one of which the $\Lambda_{c}^{+}$decayed to the $\Lambda \pi^{+}$ mode. (The other two were $p K^{-} \pi^{+}$and $\Lambda \pi^{+} \pi^{0}$.) For the $\Lambda \pi^{+}$event, the mass was determined to be $2323 \pm 42 \mathrm{MeV} / \mathrm{c}^{2}$. Using all three events, the $\Lambda_{c}^{+}$mass was measured to be $2285 \pm 23 \mathrm{MeV} / \mathrm{c}^{2}$.

\section{(3-4) Characteristics of the $\Lambda_{c}^{+}$Signal}

In order to measure some physical aspect of the signal, it is necessary to minimize the effects of the background. The method employed in this thesis is a standard and well known one. First, the $\Lambda_{c}^{+}$mass distribution (figure 3-19(a)) is plotted on a two dimensional scatter plot as a function of the physical quantity of interest. Let this quantity be denoted by $\mathcal{Z}$. The $\mathcal{Z}$ distribution is split into $N$ appropriate intervals (or "slices"). Using the Maximum Likelihood method, one measures the number of $\Lambda_{c}^{+}$charmed baryons present in each of these $N$ resultant mass plots. Since the statistics for the signal are not high, only four or five bins were used. For these fits, the mean and width of the signal were kept fixed. Finally, the acceptance losses generated by the spectrometer system were simulated by a Monte Carlo program designed to account for the major losses and biases in the data. This program is described in section (3-5) of this chapter. The effects of the spectrometer were incorporated into the data distributions to produce the final physics distributions. We proceed now to discuss the results of this process.

A table of trigger types is displayed in Table 3-5. (The triggers are defined and discussed in sections 2-4-10 and 2-4-11.) This compares the trigger bit distribution between the $\Lambda_{c}^{+}$signal in figure 3-19(a) and those events rejected as having a wellidentified recoil proton (figure 3-15) with the additional constraint that only those events in the $\Lambda_{c}^{+}$mass interval of $2.22-2.32 \mathrm{GeV} / \mathrm{c}^{2}$ were used. As expected, the great majority of the recoil proton events are associated with the diffractive Recoil 2 and 3 triggers. For the $\Lambda_{c}^{+}$events, the Recoil 2 and 3 component has been reduced and redistributed into the Recoil 4 and High $P_{T}$ triggers. This is in keeping with 
the expectation of a higher momentum transfer process in charm production and the concomitant fragmentation of the target proton.

No $\bar{\Lambda}_{c}^{-}$signal is seen in the $\left(\bar{\Lambda}, \pi^{-}\right)$channel. This suggests that the $\Lambda_{c}^{+}$is produced in association with one of the charmed mesons, e.g., the $D^{-}$or $\bar{D}^{0}$. Reconstuction of these states would be definite confirmation of this proposition. However, the small sample size and low branching ratio for any one particular decay channel do not allow one to confirm the hypothesis. However, as will be presently shown, there are other indications that are consistent with the hypothesis of associated production.

Figure 3-20(a) displays the signal strength of the $\Lambda_{c}^{+}$as a function of the photon beam energy for E516. Note that the predominant part of the signal is produced below photon energies of $100 \mathrm{GeV}$. In reference [42], it is noted that associated production of charm seems to be the dominant mode in the $40-70 \mathrm{GeV}$ energy range used in that experiment. Furthermore, the experiment of reference [38] records a similar result. In this case, a $20 \mathrm{GeV}$ photon beam, produced by backscattering of laser light from a $30 \mathrm{GeV}$ electron beam, is directed at a liquid hydrogen target. From results concerning the asymmetry of $D$ and $\bar{D}$ charm meson production, it is estimated that $(71 \pm 11 \pm 6) \%$ of the total charm cross section at the experimental beam energy of $20 \mathrm{GeV}$ is due to associated production: $\gamma p \rightarrow \Lambda_{c}^{+} \bar{D} X$. The results presented in this thesis are consistent with these claims. Furthermore, the 40-160 $\mathrm{GeV}$ range of photon beam energies available to E516 has lead to the observance of a decreasing cross section for energies above $100 \mathrm{GeV}$. This is shown in figure 3-20(a).

In Chapter 1, it was pointed out that in reference [84], a calculation of the rapidity distribution for the $\Lambda_{c}^{+}$is carried out using a first order photon gluon fusion model, assuming that the $\Lambda_{c}^{+}$is produced in association with a $\bar{D}^{0}$ meson. The resultant rapidity distribution for the charmed baryon was peaked in the negative direction. Figure 3-21(a) displays the rapidity distribution for the $\Lambda_{c}^{+}$signal found 
in this experiment. This is in concordance with the theoretical results found in reference [84].

Furthermore, one can also verify the result using the Feynman variable, $x_{F}$, which is defined as

$$
x_{F}=\frac{P_{\|}^{*}}{P_{\max }^{*}} .
$$

Here, $P_{\|}^{*}$ is the longitudinal momentum of the particle and $P_{m a x}^{*}$ is its maximum possible momentum. As with the rapidity, the variables are defined in the overall center-of-mass system of $\gamma p$. Since both rapidity and $x_{F}$ are defined in terms of the longitudinal momentum, it is to be expected that if the rapidity is peaked for negative values, then likewise the Feynman variable. Figure 3-22(a) establishes this fact. This is also consistent with a recent result found in another photoproduction experiment [41]. In this paper, the reconstruction of events with pairs of charmed particles is reported. Here it was also found that for $\left(\Lambda_{c}^{+}, \bar{D}\right)$ events, the $x_{F}$ distribution for the charmed baryons is peaked at $x_{F}<0$.

Finally, a plot of the $P_{T}^{2}$ distribution is shown in figure 3-23(a). The distribution is consistent with an exponential form found in hadroproduction experiments [129]. That is, the $P_{T}^{2}$ distribution should follow the form:

$$
\frac{d N}{d P_{T}^{2}}=N_{0} \exp \left(-b P_{T}^{2}\right)
$$

A fit produces a slope parameter $b=2.0 \pm 0.9(\mathrm{GeV} / \mathrm{c})^{-2}$. The results for the hadroproduction of $D$ mesons are consistent with a slope parameter of $b \approx 1.0-1.1$ $(\mathrm{GeV} / \mathrm{c})^{-2}[129]$.

The line fit was made using the effective variance technique of reference [120]. This method takes account of the fact that both abscissa and ordinate values have comparable errors. The usual least-squares method assumes errors only for the ordinate values. In the effective variance method, both sets of errors are used and an iterative method using the least-squares approach produces a set of slope and intercept values which rapidly converge to the final results. 


\section{(3-5) The E516 Monte Carlo}

Originally it was expected that the Fermilab version of the Monte Carlo would be used for simulating the effects of the spectrometer upon the data. For various reasons, this turned out to be an impractical choice. As it was, a preliminary version of a Monte Carlo simulation of the Tagged Photon Spectrometer already existed at the University of Toronto. The Fermilab version was set up to produce a simulated raw data tape which was subsequently passed through the E516 reconstruction code. For the Toronto version, the various neutral and charged tracks would be generated initially as a pure physical event. Subsequently, the detector layout, geometric acceptances, track reconstruction efficiencies, and magnetic field structure would be used to modify the event as seen by the spectrometer. To maintain consistency with the Fermilab version, some aspects of the event generation from the Fermilab version were incorporated into the Toronto package. A description of the Toronto version follows.

The user prescribes the type of event to be studied. For the purposes of this thesis, the event can be written as the following chain of reactions and/or decays:

$$
\begin{gathered}
\gamma+p \rightarrow X+R \\
X \rightarrow \Lambda_{c}^{+}+Y \\
\Lambda_{c}^{+} \rightarrow \Lambda+\pi^{+} \\
\Lambda \rightarrow p+\pi^{-}
\end{gathered}
$$

That is, an incident high energy photon interacts with a stationary proton to produce a massive state $X$ and a recoiling system $R$. The recoiling system was assigned a proton mass. Although $R$ is, strictly speaking, an unknown, the use of the proton mass is not inconsistent with the data. Most of the $\Lambda_{c}^{+}$signal is associated with diffractive Recoil 2 and 3 triggers. These events should have been rejected by the triggering system; instead the recoiling system must have simulated 
a proper event type to a sufficient degree so as to be allowed through the trigger filters.

The $X$ system is actually a $\Lambda_{c}^{+}$particle plus the system $Y$ which denotes the spray of additional charged and neutral particles (i.e. protons, kaons, and pions) accompanying the charmed baryon. Presumably, part of the $Y$ system contains the anti-charmed meson. As these could not be reconstructed from the data, only the more common hadrons (i.e., the final decay products of a charmed particle decay) are assumed to make up the system. To conserve baryon number and strangeness, enough protons and neutral and/or charged kaons are included in the $Y$ system to compensate for the $\Lambda_{c}^{+}$state and its decays. Finally the charmed baryon decays into the $\left(\Lambda, \pi^{+}\right)$with subsequent decay of the $\Lambda$ into the $\left(p, \pi^{-}\right)$pair.

The photon energy is generated according to a Monte Carlo simulation of the experimental photon beam energy spectrum for E516. This package was taken from the Fermilab Monte Carlo. The mass and multiplicity of the $Y$ system are generated in turn with the multiplicity simulated by the KNO distribution $[125,126]$. This is a well established description of the multiplicity distributions found in hadronic reactions. It is also identical to the one used in the Fermilab package. Using the mass of the $\Lambda_{c}^{+}+Y$ as a lower bound and the center-of-mass energy (minus the proton mass) as an upper bound, the mass $M_{X}$ of the $X$ system is generated from a distribution taken from reference [121]. The diffractive portion varies as $1 / M_{X}$ while the nondiffractive part is proportional to $M_{X}^{3}$. The diffractive portion was used initially. This missing mass is subsequently used to generate the recoiling momentum of $R$. The Mandelstam variable $t$ is generated according to the exponential distribution $\exp (-b t)$ where the slope $b=2$ is set as this is consistent with the creation of high mass states. (It is also the value used in the Fermilab version.)

The decay of the $X$ system is allowed to proceed initially according to phase space. The $\mathrm{N}$-body phase space generator is a well established package taken from a CERN library. The decays of the $\Lambda_{c}^{+}$and $\Lambda$ are allowed to proceed in the same 
fashion. The $Y$ system is made to decay according to a software package taken, once again, from the Fermilab version. All fixed target experiments display the common feature of limited transverse phase space for the resultant hadronic products of the initial state reaction. This feature is incorporated in a Monte Carlo package described in reference [127]. The transverse momentum distribution is prescribed according to the exponential form $\exp \left(-P_{r}^{2} / R^{2}\right)$ where $R=0.4(\mathrm{GeV} / \mathrm{c})^{-1}$.

The final products are tracked through the spectrometer. Geometric acceptances are imposed on the final states as well as various detection efficiencies. These include the PWC and drift chamber reconstruction efficiences as well as the those appropriate to the calorimeters. Charged tracks have their momentum and spatial coordinates smeared according to previous studies of the data. This included the effects of multiple scattering. In addition, studies of the track reconstruction [123] indicated that some charged tracks pointing through the entire forward system were reconstructed as short tracks that only traversed two or three drift chambers. This aspect was included in the simulation. As well, the number of drift chamber planes that a track traversed was also simulated according to data distributions. In this study, the Cerenkov particle identification efficiencies were taken from the data. Specifically, the $9 \%$ loss in the $\Lambda$ signal due to the proton identification cut was included in the simulation.

Furthermore, the reconstructed events were also run through the secondary vertex package used on the data as this imposed the cuts used to establish the $\Lambda$ candidates. In particular, many of those secondary vertices that decay very close to the main vertex are eliminated as they cannot be distinguished from the main vertex.

Finally, the cuts used in sections (3-2) and (3-3) above were imposed. Those cuts used to establish the $\Lambda$ signal were replaced by efficiency cuts obtained from the data. Overall, there was a $15 \%$ loss in signal with the principal contributors being the proton identification and the $K_{s}^{0}$ cuts. 
As a check of the Monte Carlo, one compared various distributions with those found in the data. These include particle multiplicities, mass distributions, and momenta. In particular, the proper lifetime of the $\Lambda$ was reconstructed using the results of the simulation. Figure 3-24 displays the results. The upper data set shows the generated proper lifetime of the $\Lambda$ hyperon. From the Particle Data Book [1], one finds that the accepted value is $c \tau=7.89 \pm 0.06 \mathrm{~cm}$. From the simulated data, one obtains $7.8 \pm 0.6 \mathrm{~cm}$. Below this is the lifetime distribution reconstructed from the data using the acceptances and efficiencies found by the Monte Carlo simulation. A fit produces a value of $8.4 \pm 1.0 \mathrm{~cm}$. This is consistent with the accepted value.

As a further check, various alternative models of the missing mass and recoil proton $t$ distributions were used. Various models of the missing mass distribution have been used in the Fermilab version. Principally, one used uniform distributions in $M_{X}^{2}$ or $M_{X}^{2} / s$ as the data displayed (approximately) such behaviour. In reference [46], it is shown that the recoil proton $t$ distribution has a variable slope depending upon the missing mass with small slopes being associated with high missing masses. This is the case for the particle reaction under study as $90 \%$ of the missing mass distribution lies above $5.5 \mathrm{GeV} / \mathrm{c}^{2}$ (the Recoil 3 threshold). Variable slopes ranging from 1-10 were used. Finally, the non-diffractive component of the missing mass formula was also implemented.

The results indicated that while overall efficiencies could vary by as much as $30 \%$ of the final value, the shapes of physical distributions were insensitive to the changes imposed above. This is illustrated by the $\Lambda$ lifetime results displayed in figure 3-24.

\section{(3-6) Estimate of the $\Lambda_{c}^{+}$Cross Section}

The purpose of the following discussion is to verify if an estimate of our observed $\Lambda_{c}^{+}$cross section is consistent with other experiments. It is not meant to be an accurate determination of the actual cross section. Given the difficulty of estimating our trigger efficiency, it is impossible to determine meaningful error bars on our 
cross section estimate. The fact that we select on non-recoil events in a trigger environment based on recoil proton events is the major problem in estimating a cross section for our $\Lambda_{c}^{+}$data. Nevertheless the cross section estimate does provide some measure of comparison.

The cross section for a process is defined by the following relation:

$$
\sigma=\frac{N_{e v t s}}{N_{\gamma} \cdot N_{s c} \cdot B \cdot \epsilon} .
$$

where

$$
\begin{aligned}
N_{\text {evts }} & =\text { number of observed events } \\
N_{\gamma} & =\text { number of incident photons } \\
N_{s c} & =\text { number of scattering centers per unit area, } \\
B & =\text { branching ratio for the observed process, and } \\
\epsilon & =\text { experimental detection efficiency. }
\end{aligned}
$$

This last quantity is itself the product of three factors: (i) the trigger efficiency, (ii) the geometrical acceptance, and (iii) the reconstruction efficiency.

The quantity $N_{\gamma} \cdot N_{s c}$ is also known as the luminosity $\mathcal{L}$. The number of scattering centers can be computed from the relation

$$
N_{s c}=\frac{\rho L N_{A}}{A},
$$

where

$$
\begin{aligned}
\rho & =\text { the density of liquid } H_{2}=0.0708 \mathrm{~g} \mathrm{~cm}^{-3} \\
L & =\text { length of target }=150 \mathrm{~cm} \\
N_{A} & =\text { Avogadro's number }=6.022045 \times 10^{23} \mathrm{~mol}^{-1} \\
A & =\text { gram-atomic weight of hydrogen }=1.00797 \mathrm{~g} \mathrm{~mol}^{-1} .
\end{aligned}
$$

Recall that $N_{\gamma}$ is the number of photons that interact via the hadronic cross section of $115 \mu b$. Two principal modifications must be made. First, a live-time factor must be included to take into account the effect of dead time created by the spectrometer electronics upon the event detection rate. From reference [47], the number of live-time events is $N_{0}=9.94 \times 10^{10}$ for the $170 \mathrm{GeV}$ data sample. 
Secondly, there is attenuation of the photon beam due to the much larger (by a factor of 200) pair production cross section $\sigma_{p}$. Over an infinitesimal distance $d l$ through the target, the loss due to pair production is given by

$$
d N_{\gamma}=-N_{\gamma} \frac{\sigma_{p} \rho N_{A}}{A} d l
$$

Hence

$$
N_{\gamma}=N_{0} \exp \left[-\frac{\sigma_{p} \rho N_{A}}{A} l\right] .
$$

In this case, $N_{\gamma}$ is the number of photons that survive to a distance $l$ in the target. Hence we require the average number of such photons:

$$
\begin{aligned}
\left\langle N_{\gamma}\right\rangle & =\frac{\int_{0}^{L} N_{\gamma}(l) d l}{\int_{0}^{L} d l} \\
& =\frac{N_{0}}{N_{s c} \sigma_{p}}\left[1-\exp \left(-\sigma_{p} N_{s c}\right)\right] .
\end{aligned}
$$

For energies above $1 \mathrm{GeV}$, the pair cross section can be written to a very good approximation as [1]

$$
\sigma_{p}=\frac{7}{9} \frac{A}{L_{R} N_{A}} .
$$

The parameter $L_{R}$ is known as radiation length. It is defined as that path length over which an electron will lose all but $1 / e$ of its energy to bremsstrahlung on average. For hydrogen, $L_{R}=61.28 \mathrm{~g} / \mathrm{cm}^{2}$.

Finally, one obtains the result:

$$
\begin{aligned}
\mathcal{L} & =\left\langle N_{\gamma}\right\rangle N_{s c} \\
& =590 \mathrm{nb}^{-1} .
\end{aligned}
$$

This number has a $5 \%$ error associated with it [60].

We require the overall efficiency of detecting the $\Lambda_{c}^{+}$in the Tagged Photon Spectrometer. This was determined by use of the Monte Carlo simulation presented in section (3-5). The final number is a product of three numbers:

(1) The overall geometric and reconstruction efficiency for the forward spectrometer is $0.35 \pm 0.09$. This number is a reflection of three major effects: (i) the 
geometric acceptance of the forward system, especially the drift chambers, (ii) the charged track reconstruction efficiencies for the drift chambers, and (iii) the efficiency for reconstructing secondary neutral vertices (i.e., the $\Lambda$ ).

(2) For the recoil chamber, the overall efficiency was determined to be $0.47 \pm 0.06$. This reflects three major effects: (i) the geometric acceptance of the detector, (ii) the efficiency of the three proportional wire chambers, and (iii) the efficiency for reconstructing the recoil proton kinetic energy.

(3) The trigger efficiency is taken to be 0.48 as for Recoil $2 \& 3$ events [60]. (The error in this number for diffractive production is estimated to be \pm 0.04 .) The choosing of this value for the trigger efficiency requires some explanation as it is by no means the natural choice.

As stated in the opening paragraph of this section, the trigger efficiency is difficult, if not impossible to calculate with a proper degree of confidence. The experiment was designed to trigger both on diffractively produced charm events (Recoil 2 \& 3) with an accompanying recoil proton, as well as non-diffractive events (Recoil 4).

The identification of the recoiling proton was a crucial aspect of the Recoil $2 \&$ 3 triggers. This implies a model dependent choice of trigger mechanism with respect to charm production. As has been demonstrated, the $\Lambda_{c}^{+}$charmed baryon sample presented in this thesis has been produced in an event category that should have been excluded by the trigger. Thus a recoiling fragment from the charmed baryon event emulated a recoil proton in the trigger system. Subsequent offline analysis showed that the charged track was not consistent with being a proton. Hence a proper determination of the trigger efficiency would require a model dependent analysis of the recoil and trigger systems when such non-diffractive charm events are generated in $\gamma p$ interactions. It is important to emphasize that our goal is to obtain some 'ball-park' estimate of the cross section with which to compare to other photoproduction results. As will be seen shortly, the error on the branching ratio 
for the decay mode $\Lambda_{c}^{+} \rightarrow \Lambda \pi^{+}$generates a very large error on any cross section calculation even if the other numbers in the calculation are well determined. Given that the majority of the signal was seen in the Recoil $2 \& 3$ triggers (see Table 3-5), and that the event emulated a diffractive type of event at the trigger level, it was decided that the trigger efficiency for Recoil $2 \& 3$ events with a recoiling proton would, at the very least, provide a useful upper limit on the actual trigger efficiency.

The final efficiency is the product of the above numbers: $0.079 \pm 0.024$. Finally, two branching ratios must be included for the final calculation [1]. For the decay $\Lambda \rightarrow p+\pi^{-}$, the branching ratio is $0.642 \pm 0.005$. The branching ratio for the decay of the charmed baryon $\Lambda_{c}^{+} \rightarrow \Lambda+\pi^{+}$is $0.006 \pm 0.005$. Note the large error for this latter number.

Excluding the $\Lambda_{c}^{+}$branching ratio, one determines the cross section (times branching ratio) as

$$
\begin{aligned}
\sigma B & \approx \frac{51 \pm 14}{(590 \pm 30) \cdot(0.079 \pm 0.024) \cdot(0.642 \pm 0.005)} \\
& \approx 1.7 \pm 0.6 \mathrm{nb} .
\end{aligned}
$$

Given the aforementioned qualifications, one must bear in mind that the above uncertainty could be larger if a realistic determination of the uncertainty in the trigger efficiency was possible.

A previous result from E516 [49], which does not see a $\Lambda_{c}$ signal, gives an upper limit of $\sigma B\left(\Lambda_{c}^{+} \rightarrow \Lambda \pi^{+}\right)<9 \mathrm{nb}$. This was measured for a data sample of Recoil 2 $\& 3$ events with a single well-identified recoil proton.

Inclusion of the charmed baryon branching ratio gives a cross section of $280 \pm$ $260 \mathrm{nb}$. We can compare this result with those of earlier photoproduction experiments. In reference [41], the cross section for the associated production of $\bar{D} \Lambda_{c}^{+}$ is estimated to be $64 \pm 34 \mathrm{nb}$. Unfortunately, the reconstructed decay modes for the $\Lambda_{c}^{+}$are not specified in this preprint. Reference [38] gives an estimate for the same production process as $44 \pm 9_{-8}^{+11} \mathrm{nb}$. This cross section was calculated on the idea that any asymmetry in the relative numbers of $D$ versus $\bar{D}$ was due to $\bar{D} \Lambda_{c}^{+}$ 
production. No $\Lambda_{c}^{+}$particles were actually reconstructed. The broad-band photoproduction experiment [52] estimated an upper limit to the decay mode observed in E516:

$$
\sigma B\left(\Lambda_{c}^{+} \rightarrow \Lambda \pi^{+}\right)<0.9 \mathrm{nb}
$$

This last experiment covered a similar photon beam energy range as E516, but did not have the capability of reconstructing the individual photon energies.

Finally, one can point out an earlier E516 result [57] which indicated the presence of asymmetric $D^{*+}$ and $\bar{D}^{*-}$ production for those events recorded by the Recoil 4 trigger. Recall that this trigger was supposed to be sensitive to $\Lambda_{c}$ production. An upper limit of $60 \mathrm{nb}$ was calculated as a cross section for the inclusive process:

$$
\gamma p \rightarrow \bar{D}^{*-} \Lambda_{c}^{+} X
$$

In conclusion, it seems that the cross section presented in this thesis is in accord with other results, given the error associated with it. Unfortunately, this same error disallows it from being considered as a reliable measurement. It is to be hoped that future experiments such as E691 will produce a large sample of $\Lambda_{c}$ decays (in a variety of channels) that will allow a set of precise and accurate measurements to be made. 


\section{CHAPTER 4}

\section{CONCLUSIONS}

\section{(4-1) Summary of Results}

In a fixed target experiment utilizing high energy photons incident upon a liquid hydrogen (proton) target, evidence for the charmed $\Lambda_{c}^{+}$baryon decaying into the $\left(\Lambda, \pi^{+}\right)$channel has been gathered. The signal contains $51 \pm 14$ events with a mean mass of $2270 \pm 6 \mathrm{MeV} / \mathrm{c}^{2}$, a full width at half maximum (FWHM) of $36 \pm 11 \mathrm{MeV} / \mathrm{c}^{2}$, and a statistical significance of 3.8 standard deviations.

Furthermore, there is evidence to indicate that the charmed baryon is being produced in association with a charmed meson. First of all, no signal in the corresponding anti-channel $\bar{\Lambda}_{c}^{-} \rightarrow \bar{\Lambda} \pi^{-}$is observed. Secondly, this experiment was equipped with a recoil detector that allowed the unique opportunity of analyzing the recoiling particles emitted from the $\gamma p$ interaction vertex. No part of the $\Lambda_{c}^{+}$ signal was found in those events in which a single recoil proton was emitted from the primary interaction vertex. This is indicative of a non-diffractive production mechanism.

The rapidity and $x_{F}$ distributions for the $\Lambda_{c}^{+}$in the $\gamma p$ center-of-mass frame are consistent with the theoretically derived results of reference [84]. In this model, the incident photon interacts with the target proton via a first order gluon exchange to produce a pair of charm quarks which subsequently hadronize into a $\Lambda_{c}^{+}$(or $D^{0}$ ), the $\bar{D}^{0}$ meson, and some unspecified (non-charmed) system of particles. The rapidity distribution of the charmed meson will peak in the forward (positive) direction, while in contrast, the $\Lambda_{c}^{+}$will have its peak in the backward (negative) directon (see figure 1-5).

Furthermore, as a function of the photon beam energy, the cross section for the production of the $\Lambda_{c}^{+}$is peaked for energies below $100 \mathrm{GeV}$. The experimental results of the photoproduction experiment in reference [42] indicate the predominance of 
associated charm production (over pair production) in the energy range of 40$70 \mathrm{GeV}$. Another photoproduction experiment [38] estimated that the associated production cross section is $(71 \pm 11 \pm 6) \%$ of the total charm cross section for $20 \mathrm{GeV}$ photons incident upon a proton (liquid hydrogen) target. The results presented in this thesis are qualitatively in accord with these earlier results, and in addition, indicate that the associated charm production process has a decreasing probability at higher photon beam energies.

Finally, one notes a previous result found for experiment E516 [57]. Evidence was found for the asymmetric production of $D^{*+}$ and $\bar{D}^{*-}$ mesons in Recoil $4\left(\Lambda_{c}\right.$ sensitive) triggers. (The Recoil triggers are defined and discussed in section 2-4-11.) This asymmetry was assumed to be associated with the process

$$
\gamma p \rightarrow \Lambda_{c}^{+}+\bar{D}^{*-}+\text { anything. }
$$

Separate from these results, the $P_{T}^{2}$ distribution of the $\Lambda_{c}^{+}$was found to obey an exponential law found applicable to the hadroproduction of charm [129]. A fit to the data (corrected for acceptance losses) produced a value for the slope parameter of $-2.0 \pm 0.9(\mathrm{Gev} / \mathrm{c})^{-2}$.

\section{(4-2) Comments on Charmed Baryon Physics}

Much remains to be done. The experience gained from studying the data produced in E516 has led to upgrades in the Tagged Photon Spectrometer. These improvements have been integrated into the E691 experiment (the successor to E516). In this experiment, the recoil detector has been removed and replaced with a silicon microstrip detector. Unfortunately, this means that the recoil system cannot be studied. On the other hand, the trigger will no longer be biased against the $\Lambda_{c}^{+}$ (and perhaps other higher mass charmed baryon states). More importantly, the new vertex detector allows for precision vertex position resolution $(\approx 20 \mu \mathrm{m})$ that would be simply impossible for E516 to duplicate with the recoil detector. As an added aid to reconstructing charged forward tracks, additional drift chamber planes have 
been added to the spectrometer. Improved charged particle identification was implemented by adding more mirrors to the Čerenkov counters as well as using newer, more efficient mirrors. The early results of this upgraded spectrometer (based on $15 \%$ of the data sample) are highly successful. Very large charm signals on small backgrounds are seen. In addition, several hundred events have been reconstructed in which both of the charm particles are reconstructed [130].

An important goal will be to obtain relatively large $\Lambda_{c}$ signals in several decay modes. Some possibilities are discussed in reference [88]. For example, some decays such as $\Lambda_{c}^{+} \rightarrow \Xi^{0} K^{+}$or $\Lambda_{c}^{+} \rightarrow \Delta^{++} K^{-}$are possible only through exchange graphs (see figure 1-2(b)). Furthermore, if it is possible that the $\Lambda_{c}$ is actually a two state system (with respect to mass) then decays such as the above can occur only for one of those two states due to Cabibbo suppression of one of the two possible diquark systems.

Last but certainly not least, most of the higher mass charm baryons have yet to be seen in any significant quantity (if at all). Since many of the decays produce strange baryons, it will be necessary to reconstruct these strange particles in order to finally reconstruct the initial charm baryon states-an analogous process to the one used in this thesis. Clearly, there is much spectroscopic work yet to be done. With its inherent qualities of good signal to noise and particle production rates, photoproduction experiments will continue to be a source of important data concerning charmed baryon physics in the years to come. 


\section{APPENDIX A}

\section{Some Kinematics of the $\Lambda$ Decay}

In the decay $\Lambda \rightarrow p+\pi^{-}$, let the $\Lambda$ be particle $1, p$ is particle 2 , and the $\pi^{-}$is particle 3 . Let the rest frame of the $\Lambda$ be denoted by the superscript *. Four-vectors will be in the form $\left(E, P_{x}, P_{y}, P_{z}\right)$. We will consider the $\Lambda$ decay to take place in the $x y$ plane. Hence in the CM frame, we have

$$
\begin{aligned}
& k_{1}^{*}=\left(m_{1}, 0,0,0\right), \\
& k_{2}^{*}=\left(\epsilon_{2}^{*}, k^{*} \cos \theta^{*}, k^{*} \sin \theta^{*}, 0\right), \\
& k_{3}^{*}=\left(\epsilon_{3}^{*},-k^{*} \cos \theta^{*},-k^{*} \sin \theta^{*}, 0\right),
\end{aligned}
$$

where

$$
k^{*}=\frac{\left[\left(m_{1}^{2}-\left(m_{2}+m_{3}\right)^{2}\right)\left(m_{1}^{2}-\left(m_{2}-m_{3}\right)^{2}\right)\right]^{\frac{1}{2}}}{2 m_{1}} .
$$

Transform back to the laboratory frame (moving with velocity $-\beta \hat{\mathbf{x}}$ with respect to the CM frame) using the Lorentz transformations:

$$
\begin{gathered}
\left(\begin{array}{c}
E \\
P_{\|}
\end{array}\right)=\left(\begin{array}{cc}
\gamma & \gamma \beta \\
\gamma \beta & \gamma
\end{array}\right)\left(\begin{array}{l}
E^{*} \\
P_{\|}^{*}
\end{array}\right), \\
P_{\perp}=P_{\perp}^{*} .
\end{gathered}
$$

One obtains

$$
\begin{aligned}
k_{1} & =\left(\gamma m_{1}, \gamma \beta m_{1}, 0,0\right) \\
& =\left(E_{1}, P_{1}, 0,0\right) .
\end{aligned}
$$

Thus $\gamma=E_{1} / m_{1}$ and $\gamma \beta=P_{1} / m_{1}$. Substitution in the Lorentz formulae yields the following results:

$$
\begin{aligned}
E_{2} & =\frac{1}{m_{1}}\left[E_{1} \epsilon_{2}^{*}+P_{1} k^{*} \cos \theta^{*}\right] \\
P_{2 x} & =\frac{1}{m_{1}}\left[P_{1} \epsilon_{2}^{*}+E_{1} k^{*} \cos \theta^{*}\right] \\
P_{2 y} & =k^{*} \sin \theta^{*} \\
P_{2 z} & =0
\end{aligned}
$$




$$
\begin{aligned}
E_{3} & =\frac{1}{m_{1}}\left[E_{1} \epsilon_{3}^{*}-P_{1} k^{*} \cos \theta^{*}\right] \\
P_{3 x} & =\frac{1}{m_{1}}\left[P_{1} \epsilon_{3}^{*}-E_{1} k^{*} \cos \theta^{*}\right] \\
P_{3 y} & =-k^{*} \sin \theta^{*} \\
P_{3 z} & =0 .
\end{aligned}
$$

Instead of showing that the proton must have a larger momentum than the pion, we will investigate under what conditions the proton can actually have a smaller momentum than that of the pion. Clearly, only the $x$ components need to be examined. Noting that $\epsilon_{i}^{*}=\sqrt{k^{* 2}+m_{i}^{2}}$, one obtains after some algebraic rearrangement the following relation:

$$
\frac{\sqrt{P_{1}^{2}+m_{1}^{2}}}{P_{1}} \cos \theta^{*}<\frac{\sqrt{k^{* 2}+m_{3}^{2}}-\sqrt{k^{* 2}+m_{2}^{2}}}{2 k^{*}} .
$$

Substitution of the Particle Data Book [1] values for the respective particle masses yields the result:

$$
\frac{E_{1}}{P_{1}} \cos \theta^{*}<-3.86 .
$$

First, this result requires that $\frac{\pi}{2}<\theta^{*} \leq \pi$. For $\theta^{*}=\pi$, one finds that $P_{1}<300$ $\mathrm{MeV} / \mathrm{c}$, and furthermore, this threshold momentum approaches zero as $\theta^{*} \rightarrow \frac{\pi}{2}$. Hence for $\Lambda 3$-momenta above $300 \mathrm{MeV} / \mathrm{c}$, the proton will always have the greater share of the final momentum. 
Table 1-1

THE FUNDAMENTAL PARTICLES

(I) Leptons

\begin{tabular}{lcrrrr}
\hline \multicolumn{1}{c}{ Name } & Mass & Charge & \multicolumn{3}{c}{ Lepton Numbers } \\
\multicolumn{1}{c}{ and Symbol } & $\left(\mathrm{GeV} / \mathrm{c}^{2}\right)$ & $\mathrm{Q}$ & $L_{e}$ & $L_{\mu}$ & $L_{\tau}$ \\
\hline Electron, $e$ & $\left(\begin{array}{c}0.5110034 \\
\pm 0.0000014\end{array}\right) \times 10^{-3}$ & -1 & 1 & 0 & 0 \\
Electron neutrino, $\nu_{e}$ & $0\left(<0.46 \times 10^{-7}\right)$ & 0 & 1 & 0 & 0 \\
Muon, $\mu$ & 0.10565916 & -1 & 0 & 1 & 0 \\
Muon neutrino, $\nu_{\mu}$ & $0(<0.00000030$ & 0 & 0 & 1 & 0 \\
Tau, $\tau$ & $1.7842 \pm 0.0032$ & -1 & 0 & 0 & 1 \\
Tau neutrino, $\nu_{\tau}$ & $0(<0.070)$ & 0 & 0 & 0 & 1 \\
\hline
\end{tabular}

(II) Quarks

\begin{tabular}{lcccccc}
\hline $\begin{array}{l}\text { Quark } \\
\text { flavour }\end{array}$ & $\begin{array}{c}u \\
\text { up }\end{array}$ & $\begin{array}{c}d \\
\text { down }\end{array}$ & $\begin{array}{c}s \\
\text { strange }\end{array}$ & $\begin{array}{c}c \\
\text { charm }\end{array}$ & $\begin{array}{c}b \\
\text { beauty }\end{array}$ & $\begin{array}{c}t \\
\text { truth }\end{array}$ \\
\hline Baryon number & $1 / 3$ & $1 / 3$ & $1 / 3$ & $1 / 3$ & $1 / 3$ & $1 / 3$ \\
Spin & $1 / 2$ & $1 / 2$ & $1 / 2$ & $1 / 2$ & $1 / 2$ & $1 / 2$ \\
Charge & $2 / 3$ & $-1 / 3$ & $-1 / 3$ & $2 / 3$ & $-1 / 3$ & $2 / 3$ \\
Isospin $I$ & $1 / 2$ & $1 / 2$ & 0 & 0 & 0 & 0 \\
$I_{3}$ & $+1 / 2$ & $-1 / 2$ & 0 & 0 & 0 & 0 \\
Strangeness & 0 & 0 & -1 & 0 & 0 & 0 \\
Charm & 0 & 0 & 0 & 1 & 0 & 0 \\
Beauty & 0 & 0 & 0 & 0 & 1 & 0 \\
Truth & 0 & 0 & 0 & 0 & 0 & 1 \\
\hline
\end{tabular}

Quark Masses $\left(\mathrm{GeV} / \mathrm{c}^{2}\right)$ [116]

\begin{tabular}{cccccc}
\hline$u$ & $(5.1 \pm 1.5) \times 10^{-3}$ & $d$ & $(8.9 \pm 2.6) \times 10^{-3}$ & $s$ & $0.175 \pm 0.55$ \\
$c$ & $1.27 \pm 0.05$ & $b$ & $4.25 \pm 0.10$ & $t$ & $?(>15)$ \\
\hline
\end{tabular}

(III) Gauge Bosons

\begin{tabular}{lcc}
\hline $\begin{array}{c}\text { Name } \\
\text { and Symbol }\end{array}$ & $\begin{array}{c}\text { Mass } \\
\left(\mathrm{GeV} / c^{2}\right)\end{array}$ & Charge \\
\hline Photon, $\gamma$ & $0\left(<3 \times 10^{-36}\right)$ & 0 \\
W bosons, $W^{ \pm}$ & $81.8 \pm 1.5$ & \pm 1 \\
Z boson, $Z^{0}$ & $92.6 \pm 1.7$ & 0 \\
Gluon, $g$ & 0 & 8 colour states \\
\hline
\end{tabular}


Table 1-2

Charmed Baryon Production Results

(1975-1986)

\begin{tabular}{|c|c|c|c|c|}
\hline Ref. & $\begin{array}{l}\text { Initial } \\
\text { state }\end{array}$ & $\begin{array}{c}\text { Charmed baryon } \\
\text { decays }\end{array}$ & $\begin{array}{c}\text { Mass } \\
\left(\mathrm{MeV} / c^{2}\right)\end{array}$ & $\begin{array}{l}\text { Number of } \\
\text { Events }\end{array}$ \\
\hline $\begin{array}{l}\text { Cazzoli } \star \\
{[2]}\end{array}$ & $\nu p$ & $\begin{array}{c}\Sigma_{c}^{++} \rightarrow \Lambda_{c}^{+} \pi^{+} \\
\Lambda_{c}^{+} \rightarrow \Delta \pi^{+} \pi^{+} \pi^{-}\end{array}$ & $\begin{array}{c}m\left(\Sigma_{c}^{++}\right)=2426 \pm 12 \\
m\left(\Lambda_{c}^{+}\right)=2260 \pm 20 \\
m\left(\Sigma_{c}^{++}\right)-m\left(\Lambda_{c}^{+}\right)=166 \pm 15\end{array}$ & 1 \\
\hline$\underset{[3] \bullet}{\text { Knapp }}$ & $\gamma C$ & $\begin{array}{c}\bar{\Lambda}_{c}^{-} \rightarrow \bar{\Lambda}^{-} \pi^{-} \pi^{+} \\
\text {some } \bar{\Lambda}_{c} \text { from excited } \\
\text { states at } 2.5 \mathrm{GeV} / c^{2}\end{array}$ & $m\left(\bar{\Lambda}_{c}^{-}\right)=2260 \pm 10$ & 60 \\
\hline $\begin{array}{c}\text { Barish } \star \\
{[5]}\end{array}$ & $\nu p$ & $\begin{array}{c}\Sigma_{c}^{++} \rightarrow \Lambda_{c}^{+} \pi^{+} \\
\Lambda_{c}^{+} \rightarrow p \pi^{-} \pi^{0} \bar{K}^{0} e^{+} \nu_{e}\end{array}$ & $\begin{array}{c}m\left(\Sigma_{c}^{++}\right)=2426 \pm 12 \\
m\left(\Lambda_{c}^{+}\right)>2248\end{array}$ & 1 \\
\hline $\begin{array}{l}\text { Angelini } \\
{[7]}\end{array}$ & $\nu p$ & $\Lambda_{c}^{+} \rightarrow p K^{-} \pi^{+}$ & $m\left(\Lambda_{c}^{+}\right)=2295 \pm 15$ & 1 \\
\hline $\begin{array}{c}\text { Cnops } \\
{[8]}\end{array}$ & $\nu(p, n)$ & $\begin{array}{c}\Lambda_{c}^{+} \rightarrow p K^{*-}(892) \pi^{+} \\
K^{*-} \rightarrow \bar{K}^{0} \pi^{-}\end{array}$ & $m\left(\Lambda_{c}^{+}\right)=2254 \pm 12$ & 1 \\
\hline $\begin{array}{l}\text { Baltay } \star \\
{[9]}\end{array}$ & $\nu\left(\mathrm{Ne}-\mathrm{H}_{2}\right)$ & $\begin{array}{c}\Sigma_{c}^{++} \rightarrow \Lambda_{c}^{+} \pi^{+} \\
\Lambda_{c}^{+} \rightarrow \Lambda \pi^{+}\end{array}$ & $\begin{array}{c}m\left(\Lambda_{c}^{+}\right)=2257 \pm 10 \\
m\left(\Sigma_{c}^{++}\right)-m\left(\Lambda_{c}^{+}\right)=168 \pm 3\end{array}$ & 8 \\
\hline $\begin{array}{c}\text { Drijard } \\
{[10]}\end{array}$ & $p p$ & $\Lambda_{c}^{+} \rightarrow p K^{-} \pi^{+}$ & $m\left(\Lambda_{c}^{+}\right) \sim 2260$ & \\
\hline $\begin{array}{l}\text { Giboni* } \\
{[11]}\end{array}$ & $p p$ & $\Lambda_{c}^{+} \rightarrow p K^{-} \pi^{+}$ & $m\left(\Lambda_{c}^{+}\right)=2262 \pm 10$ & 30 \\
\hline $\begin{array}{l}\text { Lockman } \\
\quad[12]\end{array}$ & $p p$ & $\begin{array}{c}\Lambda_{c}^{+} \rightarrow p K^{-} \pi^{+} \\
\Lambda_{c}^{+} \rightarrow \Lambda \pi^{+} \pi^{+} \pi^{-}\end{array}$ & $m\left(\Lambda_{c}^{+}\right) \sim 2280-2290$ & \\
\hline $\begin{array}{l}\text { Abrams } \\
{[13]}\end{array}$ & $e^{+} e^{-}$ & $\Lambda_{c} \rightarrow p K^{-} \pi^{+}+(c . c)$. & $m\left(\Lambda_{c}\right)=2285 \pm 6$ & $39 \pm 8$ \\
\hline $\begin{array}{l}\text { Allasia } \\
\text { [14] }\end{array}$ & $\nu p$ & $\Lambda_{c}^{+} \rightarrow p K^{-} \pi^{+}$ & $m\left(\Lambda_{c}^{+}\right)=2260 \pm 20$ & 1 \\
\hline $\begin{array}{l}\text { Calicchio } \\
{[15]}\end{array}$ & $\nu p$ & $\begin{array}{l}\Sigma_{c}^{+} \rightarrow \Lambda_{c}^{+}+\pi^{0} \\
\Lambda_{c}^{+} \rightarrow p K^{-} \pi^{+}\end{array}$ & $\begin{array}{c}m\left(\Sigma_{c}^{+}\right)=2457 \pm 4 \\
m\left(\Lambda_{c}^{+}\right)=2290 \pm 3 \\
m\left(\Sigma_{c}^{+}\right)-m\left(\Lambda_{c}^{+}\right)=168 \pm 3\end{array}$ & 1 \\
\hline
\end{tabular}


Table 1-2 (cont'd)

Charmed Baryon Production Results

(1975-1986)

\begin{tabular}{|c|c|c|c|c|}
\hline Ref. & $\begin{array}{l}\text { Initial } \\
\text { state }\end{array}$ & $\begin{array}{c}\text { Charmed baryon } \\
\text { decays }\end{array}$ & $\begin{array}{c}\text { Mass } \\
\left(\mathrm{MeV} / c^{2}\right)\end{array}$ & $\begin{array}{l}\text { Number of } \\
\text { Events }\end{array}$ \\
\hline $\begin{array}{l}\text { Kitagaki } \star \\
{[16]}\end{array}$ & $\nu d$ & $\begin{array}{l}\Lambda_{c}^{+} \rightarrow \Lambda \pi^{+} \\
\Lambda_{c}^{+} \rightarrow \bar{K}^{0} p\end{array}$ & $m\left(\Lambda_{c}^{+}\right)=2275 \pm 10$ & $19 \pm 7$ \\
\hline $\begin{array}{l}\text { Ushida } \\
\text { [17] }\end{array}$ & $\begin{array}{l}(\nu, \bar{\nu}) \\
\text { emulsion }\end{array}$ & $\begin{array}{l}\Lambda_{c}^{+} \rightarrow \Lambda \pi^{+} \pi^{+} \pi^{-} \\
\Lambda_{c}^{+} \rightarrow K^{-} p \pi^{+} \pi^{0} \\
\Lambda_{c}^{+} \rightarrow p \pi^{+} \pi^{-} \bar{K}^{0}\end{array}$ & $m\left(\Lambda_{c}^{+}\right)=2285$ assumed & $\begin{array}{l}2 \\
1 \\
1\end{array}$ \\
\hline $\begin{array}{c}\text { Basile } \\
{[18]}\end{array}$ & $p p$ & $\Lambda_{c}^{+} \rightarrow p K^{-} \pi^{+}$ & $\begin{array}{c}\text { study of } \bar{K}^{* 0} \rightarrow K^{-} \pi^{-} \\
\Delta^{++} \rightarrow p \pi^{+} \text {contribu }\end{array}$ & \\
\hline $\begin{array}{c}\text { Forino } \\
{[20] \bullet}\end{array}$ & $\stackrel{\gamma}{\gamma}$ emulsion & $\begin{array}{c}\gamma p \rightarrow \Lambda_{c}^{+}+\bar{D}^{0} \\
\Lambda_{c}^{+} \rightarrow \Lambda \pi^{+} \\
\bar{D}^{0} \rightarrow K^{+} \pi^{-} \pi^{-} \pi^{+}\end{array}$ & $m\left(\Lambda_{c}^{+}\right)=2330 \pm 50$ & $1^{*}$ \\
\hline $\begin{array}{c}\text { Grässler } \\
{[22]}\end{array}$ & $\nu p$ & $\Lambda_{c}^{+} \rightarrow p K^{-} \pi^{+}$ & $\begin{array}{l}2285 \pm 5 \\
2280 \pm 3\end{array}$ & 2 \\
\hline $\begin{array}{l}\text { Irion॰ } \\
{[23]}\end{array}$ & $p p$ & $\begin{array}{l}\Lambda_{c}^{+} \rightarrow p K^{-} \pi^{+} \\
\bar{\Lambda}_{c}^{-} \rightarrow \bar{p} K^{+} \pi^{-}\end{array}$ & $\begin{array}{l}2262 \pm 10 \\
2245 \pm 10\end{array}$ & \\
\hline $\begin{array}{l}\text { Russell» } \\
{[24] \bullet}\end{array}$ & $\gamma C$ & $\Lambda_{c} \rightarrow \bar{K}_{s}^{0} p+(c . c)$. & $2284 \pm 5$ & $55 \pm 10$ \\
\hline $\begin{array}{l}\text { Aleev } \\
{[25]}\end{array}$ & $n C$ & $\Lambda_{c}^{+} \rightarrow p \bar{K}_{s}^{0} \pi^{+} \pi^{-}$ & $2259 \pm 20$ & 76 \\
\hline $\begin{array}{l}\text { Bosetti* } \\
{[26]}\end{array}$ & $\nu p$ & $\begin{array}{l}\Sigma_{c}^{++} \rightarrow \Lambda_{c}^{+} \pi^{+} \\
\Lambda_{c}^{+} \rightarrow p K^{-} \pi^{+}\end{array}$ & $\begin{array}{c}m\left(\Sigma_{c}^{++}\right)=2454 \pm 5 \\
m\left(\Lambda_{c}^{+}\right)=2288 \pm 5 \\
m\left(\Sigma_{c}^{++}\right)-m\left(\Lambda_{c}^{+}\right)=166 \pm 1\end{array}$ & $1 \dagger$ \\
\hline $\begin{array}{l}\text { Kitagaki* } \\
{[27]}\end{array}$ & $\nu d$ & $\begin{array}{c}\Lambda_{c}^{+} \rightarrow \Sigma^{0} \pi^{+} \\
\Sigma^{0} \rightarrow \Lambda \gamma\end{array}$ & $2270 \pm 15$ & 3 \\
\hline $\begin{array}{l}\text { Son } \\
{[28]}\end{array}$ & $\nu d$ & \multicolumn{2}{|c|}{$B . R .\left(\Lambda_{c}^{+} \rightarrow \Lambda+X\right)=(2.0 \pm 0.74 \pm 0.65) \%$} & \\
\hline $\begin{array}{l}\text { Vella } \\
{[29]}\end{array}$ & $e^{+} e^{-}$ & \multicolumn{2}{|c|}{$\begin{array}{l}\text { B.R. }\left(\Lambda_{c}^{+} \rightarrow e^{+} X\right)=(4.5 \pm 1.7) \% \\
\text { B.R. }\left(\Lambda_{c}^{+} \rightarrow p e^{+} X\right)=(1.8 \pm 0.9) \% \\
\text { B.R. }\left(\Lambda_{c}^{+} \rightarrow \Lambda e^{+} X\right)=(1.1 \pm 0.8) \%\end{array}$} & \\
\hline
\end{tabular}


Table 1-2 (cont'd)

Charmed Baryon Production Results

(1975-1986)

\begin{tabular}{|c|c|c|c|c|}
\hline Ref. & $\begin{array}{l}\text { Initial } \\
\text { state }\end{array}$ & $\begin{array}{c}\text { Charmed baryon } \\
\text { decays }\end{array}$ & $\begin{array}{c}\text { Mass } \\
\left(\mathrm{MeV} / \mathrm{c}^{2}\right)\end{array}$ & $\begin{array}{l}\text { Number of } \\
\text { Events }\end{array}$ \\
\hline $\begin{array}{l}\text { Ushida } \\
{[31]}\end{array}$ & $\begin{array}{l}\quad(\nu, \bar{\nu}) \\
\text { emulsion }\end{array}$ & $\begin{array}{c}\Lambda_{c}^{+} \rightarrow \Lambda \pi^{+} \pi^{+} \pi^{-} \\
\Lambda_{c}^{+} \rightarrow \bar{K}^{0} p \pi^{+} \pi^{-} \\
\Lambda_{c}^{+} \rightarrow K^{-} p \pi^{+} \pi^{0} \\
\quad \Lambda_{c}^{+} \rightarrow \Sigma^{0} \pi^{+}\end{array}$ & $\begin{array}{c}m\left(\Lambda_{c}^{+}\right)=2285 \text { assumed } \\
\text { weighted average } \\
\text { is } 2265 \pm 30\end{array}$ & $\begin{array}{l}3 \\
2 \\
1 \\
1\end{array}$ \\
\hline $\begin{array}{l}\text { Biagi } \\
{[32]}\end{array}$ & $\Sigma^{-B e}$ & $\Xi_{c}^{+}\left(A^{+}\right) \rightarrow \Lambda K^{-} \pi^{+} \pi^{+}$ & $m\left(\Xi_{c}^{+}\right)=2460 \pm 15$ & $82 \pm 16$ \\
\hline $\begin{array}{l}\text { Abe } \\
{[33] \bullet}\end{array}$ & $\gamma p$ & \multicolumn{3}{|c|}{$\sigma\left(\bar{D} \Lambda_{c}^{+} X\right) / \sigma($ charm $)=(35 \pm 20) \%$} \\
\hline $\begin{array}{c}\text { Adamovich } \star \\
{[34] \bullet}\end{array}$ & $\stackrel{\gamma}{\gamma}$ emulsion & $\begin{array}{c}\Lambda_{c}^{+} \rightarrow p K^{-} \pi^{+} \\
\Lambda_{c}^{+} \rightarrow \Lambda \pi^{+} \pi^{0} \\
\Lambda_{c}^{+} \rightarrow \Lambda \pi^{+}\end{array}$ & $m\left(\Lambda_{c}^{+}\right)=2285 \pm 23$ & 3 \\
\hline $\begin{array}{l}\text { Aleev } \star \\
{[35]}\end{array}$ & $n C$ & $\begin{array}{l}\Lambda_{c}^{+} \rightarrow \bar{K}_{s}^{0} p \pi^{+} \pi^{-} \\
\Lambda_{c}^{+} \rightarrow \Lambda \pi^{+} \pi^{+} \pi^{-}\end{array}$ & $2268 \pm 6 \S$ & $\begin{array}{c}130 \pm 18 \\
57 \pm 14\end{array}$ \\
\hline $\begin{array}{l}\text { Biagi } \\
{[36]}\end{array}$ & $\Sigma^{-} B e$ & $\begin{array}{c}\Xi_{c}^{+}\left(A^{+}\right) \rightarrow \Lambda K^{-} \pi^{+} \pi^{+} \\
\Omega_{c}^{0}\left(T^{0}\right) \rightarrow \Xi^{-} K^{-} \pi^{+} \pi^{+} \\
\Xi_{c}^{0}\left(A^{0}\right) \rightarrow \Lambda K^{-} \pi^{+}\end{array}$ & $\begin{array}{c}m\left(\Xi_{c}^{+}\right)=2460 \pm 15 \\
m\left(\Omega_{c}^{0}\right)=2740 \pm 20 \\
\text { searched for, but not seen }\end{array}$ & $82 \pm 16 \ddagger$ \\
\hline $\begin{array}{c}\text { Bowcock } \\
{[37]}\end{array}$ & $e^{+} e^{-}$ & $\Lambda_{c} \rightarrow \Lambda \pi^{+} \pi^{+} \pi^{-}+($c.c. $)$ & $2287 \pm 11 \pm 5$ & $108 \pm 28$ \\
\hline $\begin{array}{l}\text { Abeף } \\
{[38] \bullet}\end{array}$ & $\gamma p$ & $\sigma\left(\bar{D} \Lambda_{c}^{+} X\right) / c$ & $($ charm $)=(71 \pm 11 \pm 6) \%$ & \\
\hline $\begin{array}{l}\text { Ushidas } \\
\text { [39] }\end{array}$ & $\begin{array}{l}(\nu, \bar{\nu}) \\
\text { emulsion }\end{array}$ & $\begin{array}{c}\Lambda_{c}^{+} \rightarrow 4\left(\bar{K}^{0} p \pi^{+} \pi^{-}\right) \\
\Lambda_{c}^{+} \rightarrow 3\left(\Lambda \pi^{+} \pi^{+} \pi^{-}\right) \\
\Lambda_{c}^{+} \rightarrow 2\left(K^{-} p \pi^{+}\right) \\
\Lambda_{c}^{+} \rightarrow \bar{K}^{0} p \\
\Lambda_{c}^{+} \rightarrow \Sigma^{0} \pi^{+} \\
\Lambda_{c}^{+} \rightarrow K^{-} p \pi^{+} \pi^{0} \\
\Lambda_{c}^{+} \rightarrow \Lambda \pi^{+} \pi^{0}\end{array}$ & $\begin{array}{c}m\left(\Lambda_{c}^{+}\right)=2282 \text { assumed } \\
\text { weighted average } \\
\text { is } 2266 \pm 13 \phi\end{array}$ & 13 \\
\hline $\begin{array}{c}\text { Seywerd } \\
{[40]}\end{array}$ & $e^{+} e^{-}$ & $\begin{array}{c}\Lambda_{c} \rightarrow p K^{-} \pi^{+}+(\text {c.c. }) \\
\Lambda_{c} \rightarrow \Lambda \pi^{+} \pi^{+} \pi^{-}+(\text {c.c. })\end{array}$ & $\begin{array}{c}m\left(\Lambda_{c}\right)=2283.5 \pm 2.6 \pm 2.1 \\
m\left(\Lambda_{c}\right)=2277.3 \pm 5.5\end{array}$ & $\begin{array}{l}479 \pm 57 \\
121 \pm 35\end{array}$ \\
\hline $\begin{array}{c}\text { Adamovich } \\
{[41] \bullet}\end{array}$ & $\begin{array}{c}\gamma \\
\text { emulsion }\end{array}$ & $\begin{array}{l}\bar{D}^{0} \Lambda_{c}^{+} \\
D^{-} \Lambda_{c}^{+}\end{array}$ & $\begin{aligned} \sigma\left(\bar{D} \Lambda_{c}^{+}\right) & =64 \pm 34 \mathrm{nb} \\
\sigma(\text { charm }) & =230 \pm 57 \mathrm{nb}\end{aligned}$ & $\begin{array}{l}7 \\
5\end{array}$ \\
\hline
\end{tabular}


Table 1-2 (cont'd)

\section{Charmed Baryon Production Results}

(1975-1986)

\section{NOTES:}

- photoproduction experiment.

* mass values used in Particle Data Group (1986) [1] average of $m\left(\Lambda_{c}\right)=2281.2 \pm 3.0 \mathrm{MeV} / \mathrm{c}^{2}$.

* same event as Adamovich [21].

$\diamond$ marginal $3 \sigma \Lambda_{c}^{+}$and $2 \sigma \overline{\Lambda_{c}}$ signals.

$\dagger$ combined with earlier results of Grässler [22] to give $m\left(\Lambda_{c}^{+}\right)=2283 \pm 3 \mathrm{MeV} / \mathrm{c}^{2}$.

$\ddagger$ same sample as Biagi [32].

$\S$ supersedes earlier results of Aleev [25].

I supersedes previous result of Abe [33].

\& supersedes earlier result of Ushida [31].

- average for eight fully constrained events. 
Table 1-3

\section{Selection Rules for Charm Decay}

\begin{tabular}{cc}
\hline $\begin{array}{c}\text { Amplitude dependence } \\
\text { on } \cos \theta_{c}\end{array}$ & Selection rules \\
\hline \multicolumn{2}{c}{ 1. Leptonic or semi-leptonic modes } \\
$\cos \theta_{c}$ & $\Delta S=\Delta C=\Delta Q=-1$ \\
& $\Delta I_{3}=|\Delta \mathrm{I}|=0$ \\
$\sin \theta_{c}$ & $\Delta C=\Delta Q=-1$ \\
& $\Delta S=0$ \\
& $\Delta I_{3}=-1 / 2,|\Delta \mathrm{I}|=1 / 2$ \\
$\cos ^{2} \theta_{c}$ & 2. Hadronic modes \\
& $\Delta S=\Delta C=-1$ \\
$\cos \theta_{c} \sin \theta_{c}$ & $\Delta I_{3}=+1,|\Delta \mathrm{I}|=1$ \\
& $\Delta S=0, \Delta C=-1$ \\
$\sin ^{2} \theta_{c}$ & $\Delta I_{3}=+1 / 2,|\Delta \mathrm{I}|=1 / 2,3 / 2$ \\
& $\Delta I_{3}=0,|\Delta \mathrm{I}|=0,1$ \\
\hline
\end{tabular}

Table 1-4

Charm baryon notations

\begin{tabular}{cccc}
\hline Charm & $\begin{array}{c}\text { Quark } \\
\text { content }\end{array}$ & $\begin{array}{c}\text { Gaillard et al. } \\
{[88]}\end{array}$ & $\begin{array}{c}\text { Aguilar-Benitez et al. } \\
{[1]}\end{array}$ \\
\hline+2 & $(c c d)$ & $X_{d}^{+}$ & $\Xi_{c c}^{+}$ \\
& $(c c u)$ & $X_{u}^{++}$ & $\Xi_{c c}^{++}$ \\
& $(c c s)$ & $X_{s}^{+}$ & $\Omega_{c c}^{+}$ \\
+1 & $(c d d)$ & $C_{1}^{0}$ & $\Sigma_{c}^{0}$ \\
& $\left(c(u d)^{S, A}\right)$ & $C_{1}^{+}, C_{0}^{+}$ & $\Sigma_{c}^{+}, \Lambda_{c}^{+}$ \\
& $(c u u)$ & $C_{1}^{++}$ & $\Sigma_{c}^{++}$ \\
& $\left(c(s d)^{S, A}\right)$ & $S^{0}, A^{0}$ & $\Xi_{c}^{0(S)}, \Xi_{c}^{0(A)}$ \\
& $\left(c(s u)^{S, A}\right)$ & $S^{+}, A^{+}$ & $\Xi_{c}^{+(S)}, \Xi_{c}^{+(A)}$ \\
& $(c s s)$ & $T^{0}$ & $\Omega_{c}^{0}$ \\
\hline
\end{tabular}


Table 2-1

Čerenkov Counter Characteristics

\begin{tabular}{ccc}
\hline Quantity & $\mathrm{C} 1$ & $\mathrm{C} 2$ \\
\hline Gas mixture & $100 \% N_{2}$ & $80 \% \mathrm{He}, 20 \% N_{2}$ \\
Length & $3.7 \mathrm{~m}$. & $6.6 \mathrm{~m}$. \\
$\begin{array}{c}\text { Refractive index } \\
\text { expected for }\end{array}$ & 1.000288 & 1.000084 \\
$76 \mathrm{~cm}-\mathrm{Hg}, 20^{\circ} \mathrm{C}, \lambda=0.35 \mu \mathrm{m}$ & & \\
Refractive index from & 1.000299 & 1.000088 \\
measured $\pi^{ \pm}$thresholds & & \\
Cerenkov angle & $24 \mathrm{mrad}$ & $13 \mathrm{mrad}$ \\
$(\gamma \rightarrow \infty, \beta \rightarrow 1)$ & & \\
$e^{ \pm}$threshold & $0.021 \mathrm{GeV} / \mathrm{c}$ & $0.038 \mathrm{GeV} / \mathrm{c}$ \\
$\mu^{ \pm}$threshold & $4.3 \mathrm{GeV} / \mathrm{c}$ & $8.0 \mathrm{GeV} / \mathrm{c}$ \\
$\pi^{ \pm}$threshold & $5.7 \mathrm{GeV} / \mathrm{c}$ & $10.5 \mathrm{GeV} / \mathrm{c}$ \\
$K^{ \pm}$threshold & $20.2 \mathrm{GeV} / \mathrm{c}$ & $37.1 \mathrm{GeV} / \mathrm{c}$ \\
$p^{ \pm}$threshold & $38.3 \mathrm{GeV} / \mathrm{c}$ & $70.6 \mathrm{GeV} / \mathrm{c}$ \\
Number of cells & 20 & 20 \\
Cell sizes & $4 \times 8$ & $10 \times 18$ \\
width $\times$ height & $8 \times 8$ & $20 \times 18$ \\
(inches) & $38 \times 8$ & $65 \times 18$ \\
& $12 \times 16$ & $30 \times 32$ \\
& $38 \times 16$ & $65 \times 32$ \\
\hline & &
\end{tabular}


Table 2-2

University of Toronto $168 / \mathrm{E}$

Specifications

PDP 11/23

Bulk Memory

$168 / \mathrm{E}$

Integer

Floating point

Interface

Memory boards

Floating Point
Integer
-RT-11 and FORTH

-acts as controller for entire system

-slow 500 nsec secondary memory $-512 \mathrm{~K}$ bytes for data ( $32 \mathrm{bits} /$ word)

$-384 \mathrm{~K}$ bytes for program ( $24 \mathrm{bits} /$ word)

-TTL chip-mostly $74 \mathrm{~S}$

-200 nsec cycle time

-12 VAX size boards per $168 / \mathrm{E}$

-emulates subset of IBM 370 instructions

-2901 LSI bit slice-8 layer PC

-32 or 48 bit arithmetic

-mantissa board-8 layer PC

-exponent board-8 layer PC

$-1 / 2$ speed of IBM $370 / 168$

-addresses memory

-8 boards per $168 / E$

-55 nsec static RAM

$-128 \mathrm{~K}$ bytes data memory ( 32 bits/word)

$-96 \mathrm{~K}$ bytes program memory ( $24 \mathrm{bits} /$ word)

Benchmark Comparisons:

six $168 / \mathrm{E}=9$ VAX $11 / 780$

$\operatorname{six} 168 / E=2$ CDC $175 \mathrm{~S}$

six $168 / \mathrm{E}=1.2 \mathrm{IBM} 3033$

$\operatorname{six} 168 / E=0.75$ CDC $175 \mathrm{~S}$

$\operatorname{six} 168 / \mathrm{E}=0.60 \mathrm{IBM} 3033$ 
Table 3-1

Number of $\Lambda \rightarrow p \pi^{-}$candidates in the mass interval 1100-1136 MeV/ $\mathrm{c}^{2}$ remaining after each of the successive cuts discussed in section (3-2).

\begin{tabular}{lccc}
\hline \hline & $\Lambda$ & $\bar{\Lambda}$ & $\Lambda+\bar{\Lambda}$ \\
\hline All candidates & 259,206 & 241,345 & 500,551 \\
$\operatorname{DCA}(p, \pi)$ & 116,218 & 105,310 & 221,528 \\
Proton probability & 68,917 & 58,640 & 127,557 \\
$\operatorname{DOF}(\pi)$ & 61,614 & 52,123 & 113,737 \\
$\operatorname{DOF}(p)$ & 59,667 & 50,308 & 109,975 \\
Pion momentum & 52,704 & 44,175 & 96,879 \\
Proton momentum & 51,734 & 43,212 & 94,946 \\
DCA $(\Lambda, v t x)$ & 50,282 & 41,815 & 92,097 \\
$K_{s}^{0}$ contamination & 47,667 & 39,296 & 86,963 \\
$\Lambda$ lifetime & 45,934 & 37,624 & 83,558 \\
Electron probability & 45,062 & 36,842 & 81,904 \\
$e^{+} e^{-}$contamination & 44,857 & 36,634 & 81,491 \\
Track duplication & 43,561 & 35,569 & 79,130 \\
\hline \hline
\end{tabular}




\section{Table 3-2}

Results of Fits to the $\Lambda / \bar{\Lambda}$ signals in Figure 3-13

\begin{tabular}{lccc}
\hline \hline Category & & $\Lambda$ & $\bar{\Lambda}$ \\
\hline poor proton & $M$ & $1115.8 \pm 0.3$ & $1116.0 \pm 0.6$ \\
and pion & $\sigma$ & $4.0 \pm 0.3$ & $5.1 \pm 0.8$ \\
& $S$ & $2489 \pm 95$ & $1479 \pm 85$ \\
& & & \\
poor proton & & $1115.9 \pm 0.1$ & $1115.9 \pm 0.1$ \\
or pion & $2.9 \pm 0.1$ & $2.9 \pm 0.2$ \\
& $6014 \pm 130$ & $3745 \pm 120$ \\
good proton & & $1115.66 \pm 0.04$ & $1115.70 \pm 0.06$ \\
and pion & $2.16 \pm 0.05$ & $2.21 \pm 0.06$ \\
& & $8568 \pm 120$ & $5725 \pm 105$ \\
\hline \hline
\end{tabular}

$M$ Mean mass of signal $\left(\mathrm{MeV} / \mathrm{c}^{2}\right)$.

$\sigma \quad$ Width of signal $\left(\mathrm{MeV} / \mathrm{c}^{2}\right)$.

$S$ Number of $\Lambda / \bar{\Lambda}$ above background.

World average [1] for $M(\Lambda)=1115.60 \pm 0.05 \mathrm{MeV} / \mathrm{c}^{2}$. 
Table 3-3

$\Lambda_{c}^{+}$Search

Number of $\left(\Lambda, \pi^{+}\right)$combinations remaining after each of the successive cuts

discussed in section (3-3).

$\left(\Lambda, \pi^{+}\right)$vertex within

3,004

$2 \mathrm{~cm}$ of main vertex

$\Lambda$ mass within $6 \mathrm{MeV} / \mathrm{c}^{2}$

1,993

of mean value $\left(1115.6 \mathrm{MeV} / \mathrm{c}^{2}\right.$ )

Diffractive recoil
events excluded

Transverse momentum of $\left(\Lambda, \pi^{+}\right)$

1,005

required to be greater than $0.4 \mathrm{GeV} / \mathrm{c}$

One $\Lambda$ per event

Photon beam energy between

951 40 and $160 \mathrm{GeV}$

Note: The final sample of 951 combinations corresponds to a total of 894 events. 


\section{Table 3-4}

\section{Charged Particle Identification Code for the Recoil Detector (MFLAG)}

\begin{tabular}{|c|c|}
\hline MFLAG $=4$ & Proton identity most probable \\
\hline 3 & Proton or Pion identity possible \\
\hline 2 & Pion identity most probable \\
\hline 1 & $\begin{array}{l}\text { Pion or hard } e^{ \pm} \text {stopping in } \\
\text { the first (A) layer of scintillator }\end{array}$ \\
\hline 0 & $\begin{array}{l}\text { Unknown: } \chi^{2} \text { too large or } \\
\text { not enough information }\end{array}$ \\
\hline
\end{tabular}

Table 3-5

Percentage of Triggers set in the $\Lambda_{c}^{+}$and Recoil Proton Data Samples of Figures 3-19(a) and 3-15 respectively

\begin{tabular}{lcl}
\hline \hline Trigger type & $\Lambda_{c}^{+}$ & Recoil $p$ \\
\hline Recoil 4 & $23 \pm 9$ & $15 \pm 4 \%$ \\
Recoil 3 & $46 \pm 12$ & $64 \pm 8$ \\
Recoil 2 & $14 \pm 6$ & $18 \pm 5$ \\
Recoil 1 & 0 & $1 \pm 1$ \\
High $P_{T}$ & $14 \pm 5$ & $1 \pm 1$ \\
Dimuon & $3 \pm 3$ & $1 \pm 1$ \\
\hline \hline
\end{tabular}


(I) Mesons

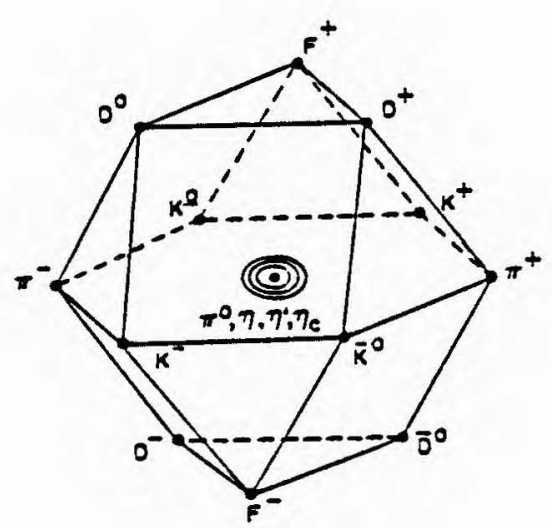

(a) $J^{P}=0^{-}$

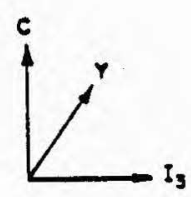

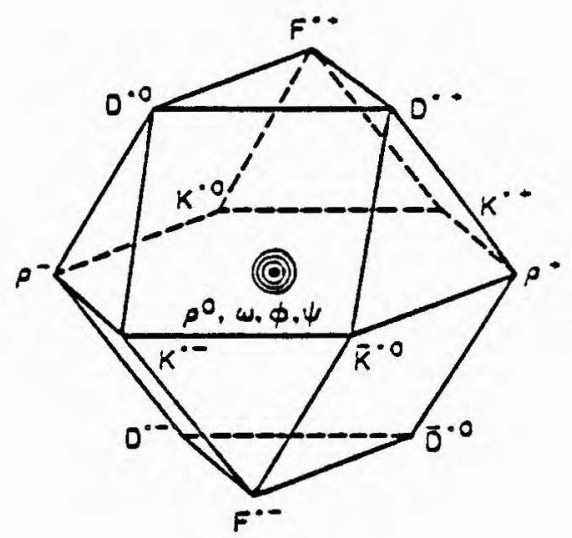

(b) $\mathrm{J}^{P}=1^{-}$

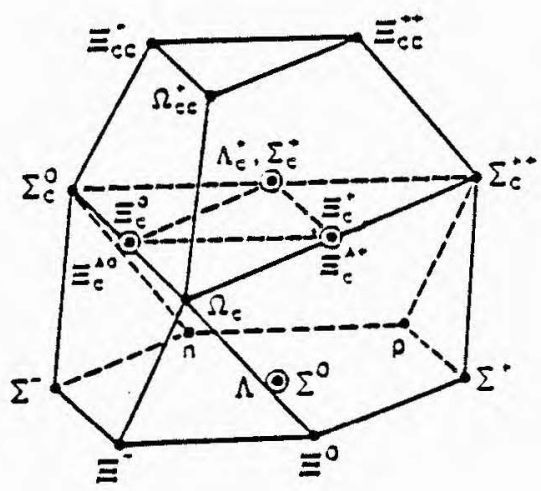

(c) $J^{P}=\frac{1}{2}^{+}$

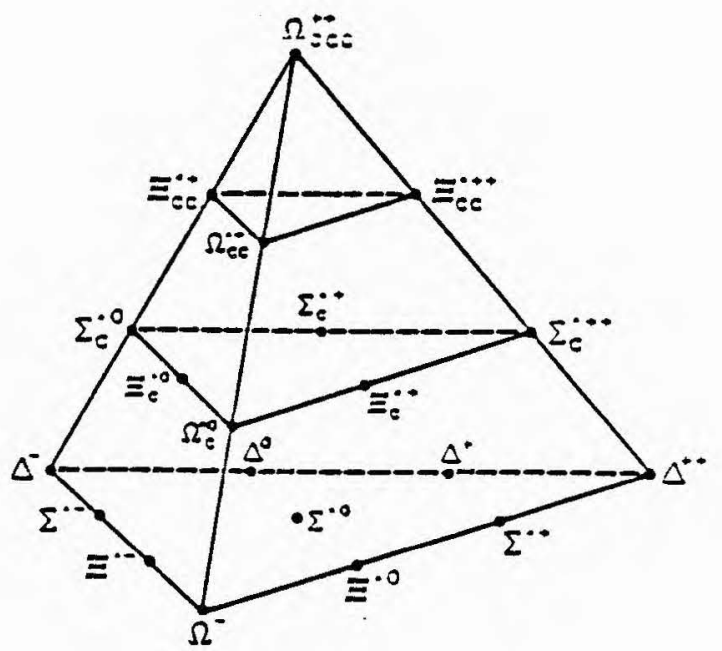

(d) $J^{P}=\frac{3}{2}^{+}$

Figure 1-1 


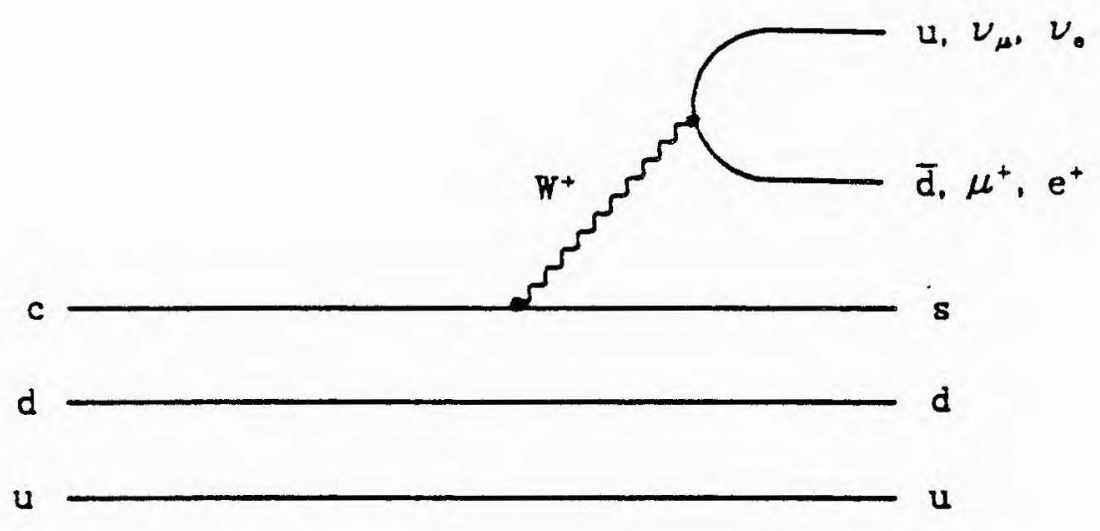

(a) Charmed baryon decay via the radiating of a $W^{+}$

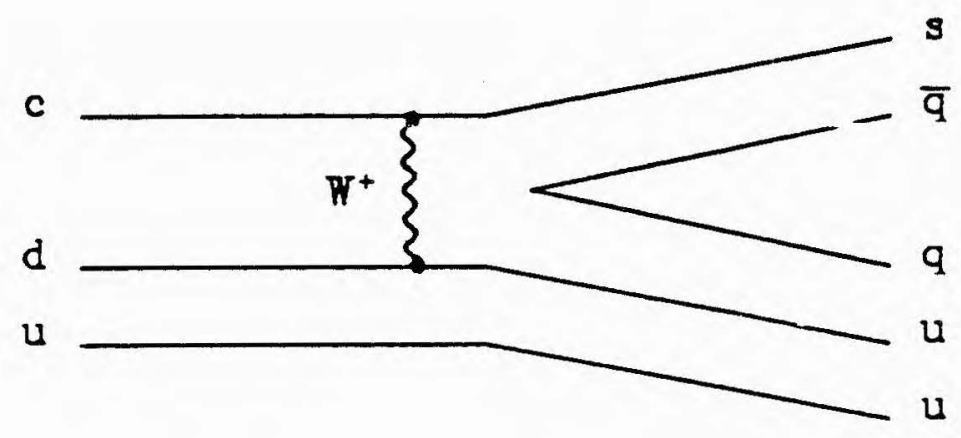

(b) Charmed baryon decay via $W^{+}$exchange

Figure 1-2 
(a) Vector Meson Dominance model for charm photoproduction.

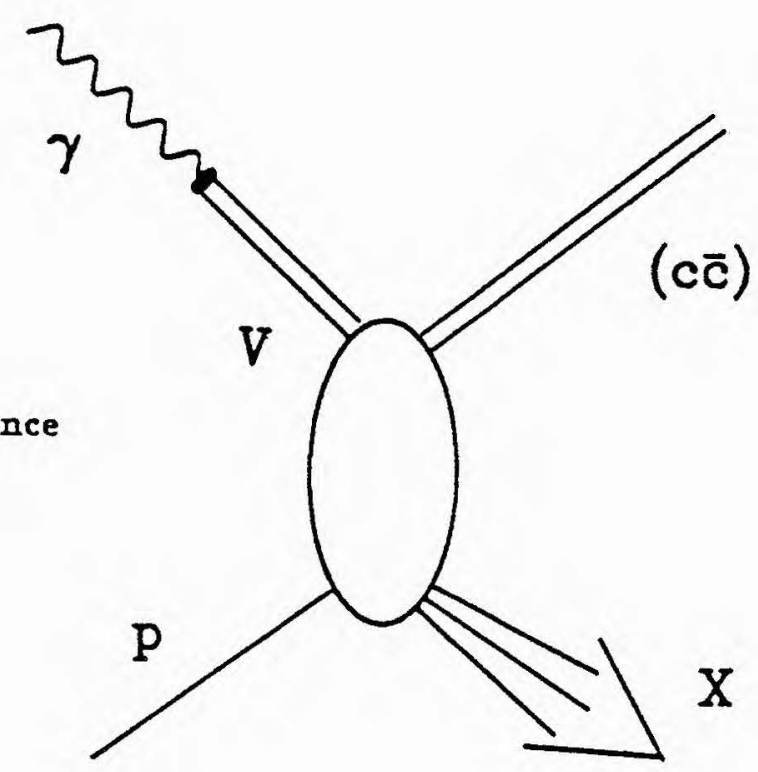

$$
3^{2}{ }^{2}
$$

(b) Photon-Gluon Fusion model (1st order).

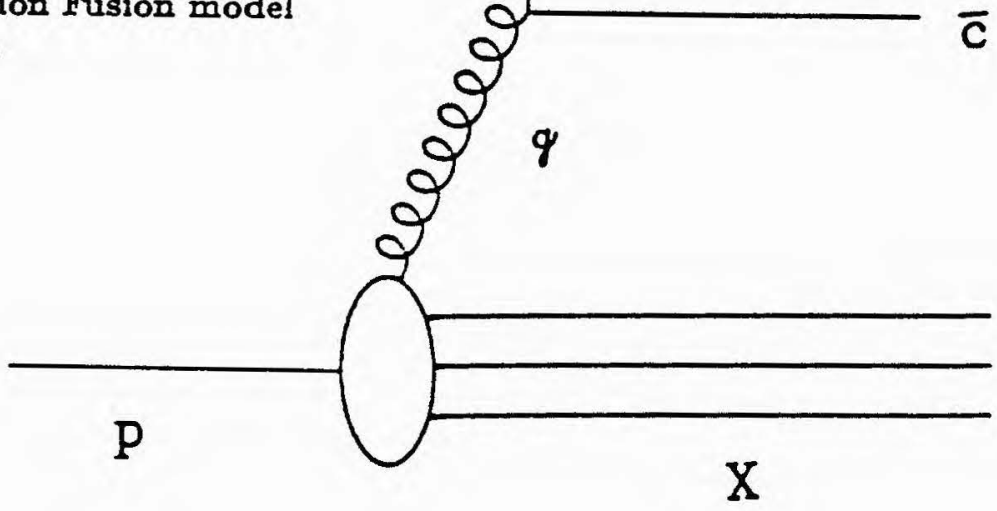

Figure 1-3 


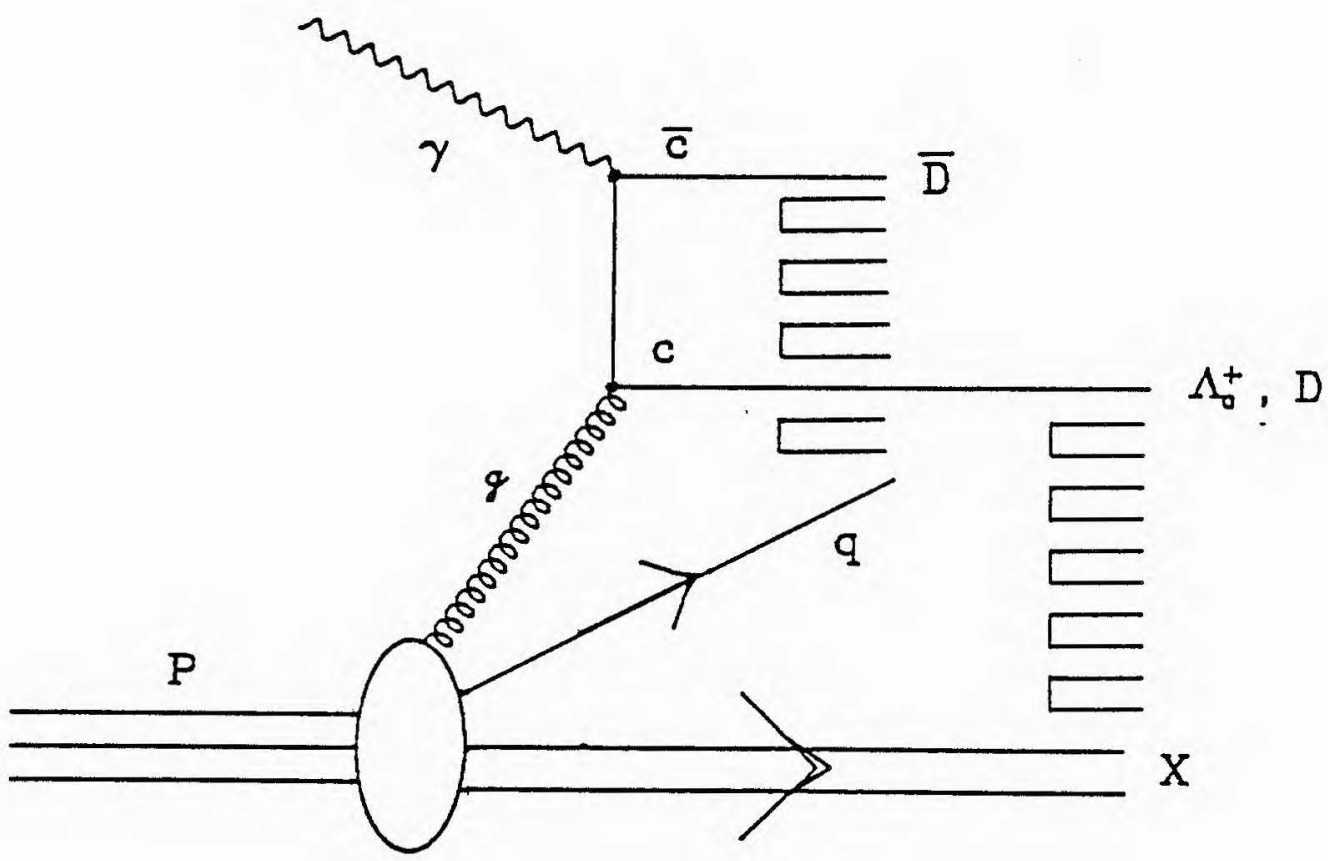

Figure 1-4 Application of first order photon-gluon fusion as calculated in reference [84]. A $\bar{c}$ quark interacts with a quark $q$ from the target proton to produce a $\bar{D}^{\circ}$ meson. The other charm quark interacts with the remaining diquark of the proton to produce a charmed meson $\left(D^{\circ}\right)$ or baryon $\left(\Lambda_{c}^{+}\right)$ plus some non-charmed system of particles $X$. 


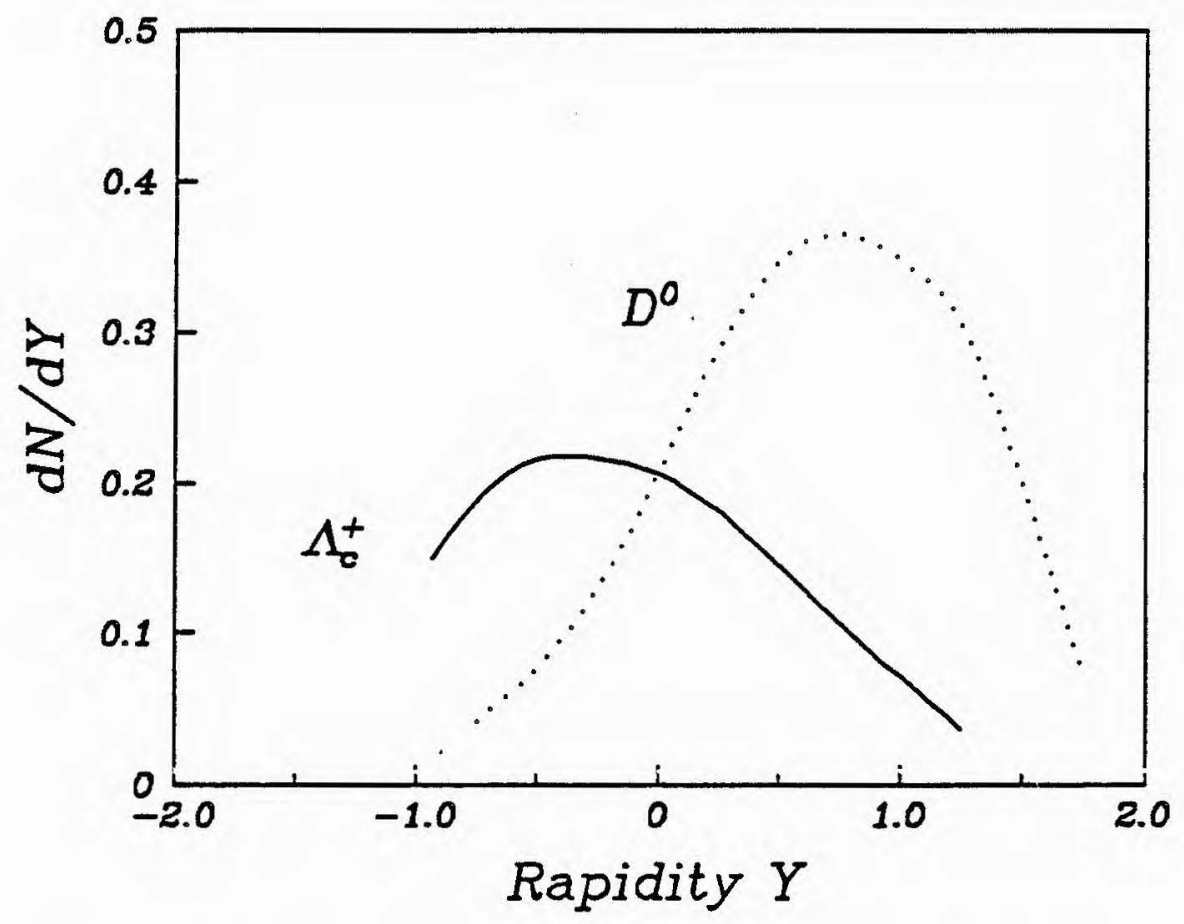

Figure 1-5 The rapidity distribution for either of the $\Lambda_{c}^{+}$or the $D^{0}$ produced at a photon beam energy of $E_{\gamma}=100 \mathrm{GeV}$. The curves are taken from reference [84]. The associated anti-charmed particle is assumed to be the $\bar{D}^{\circ}$. 

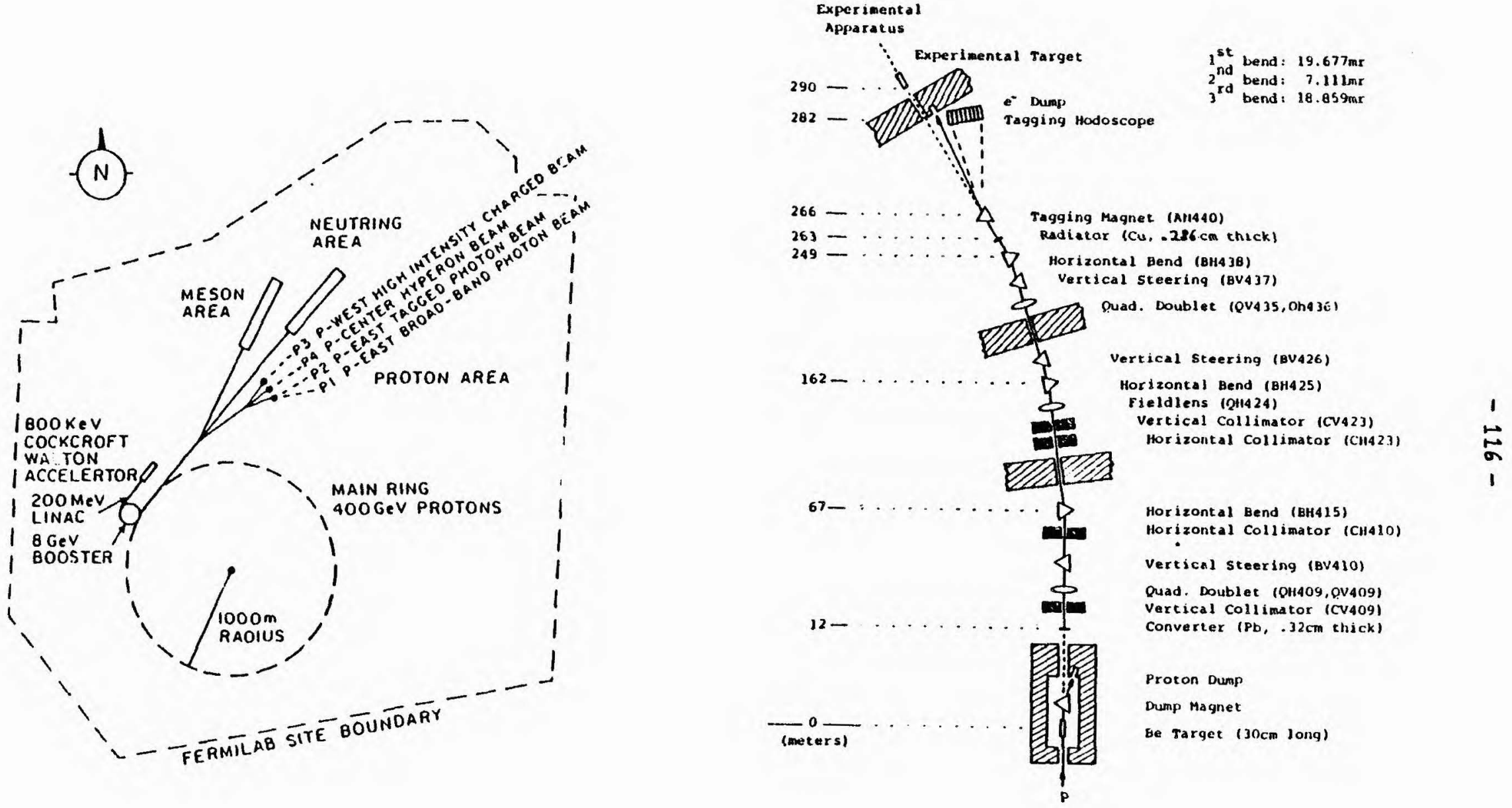

Figure 2-1 The Fermilab accelerator facility.

Figure 2-2 Schematic of the electron beam line. 


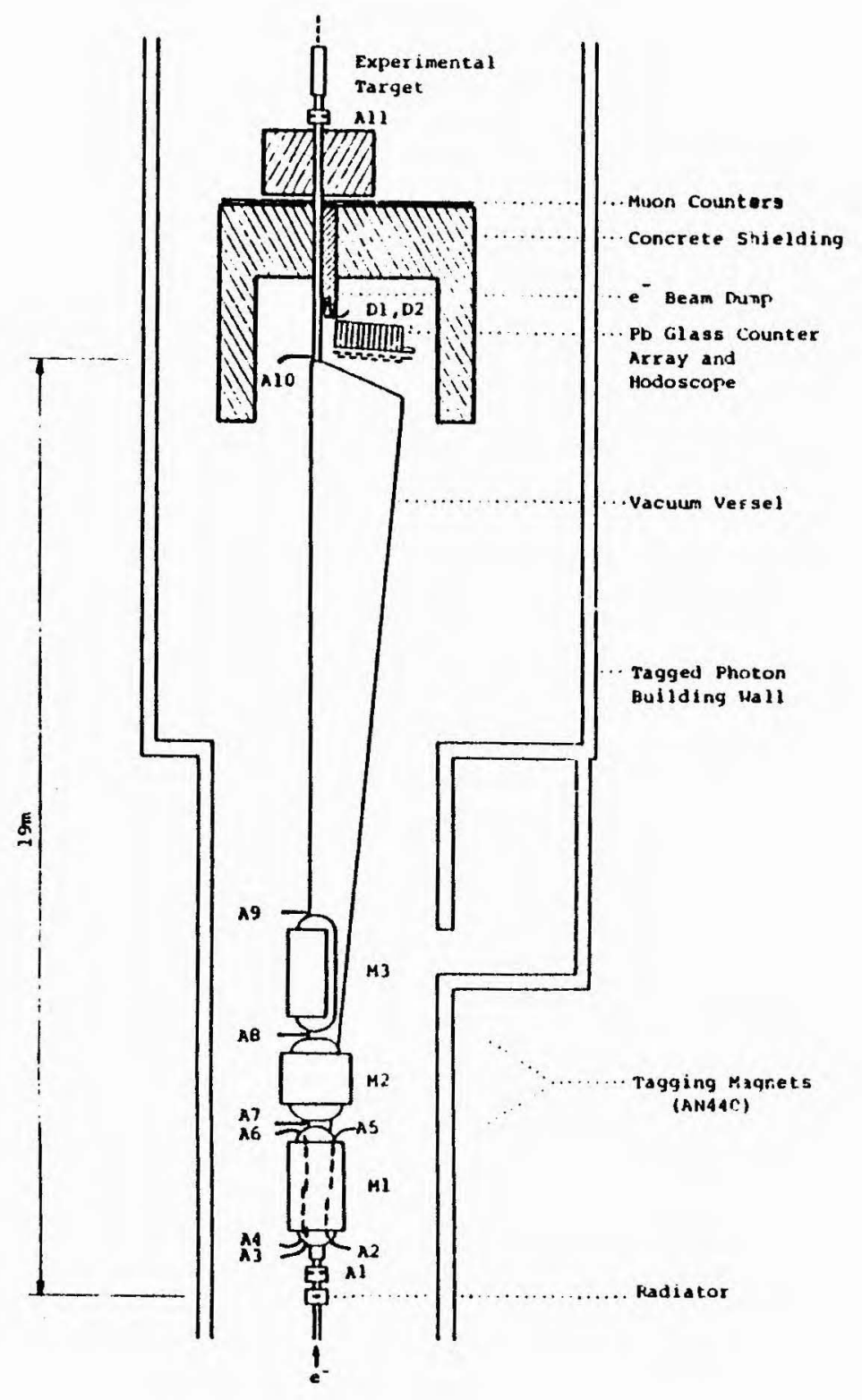

Figure 2-3 The tagging system.

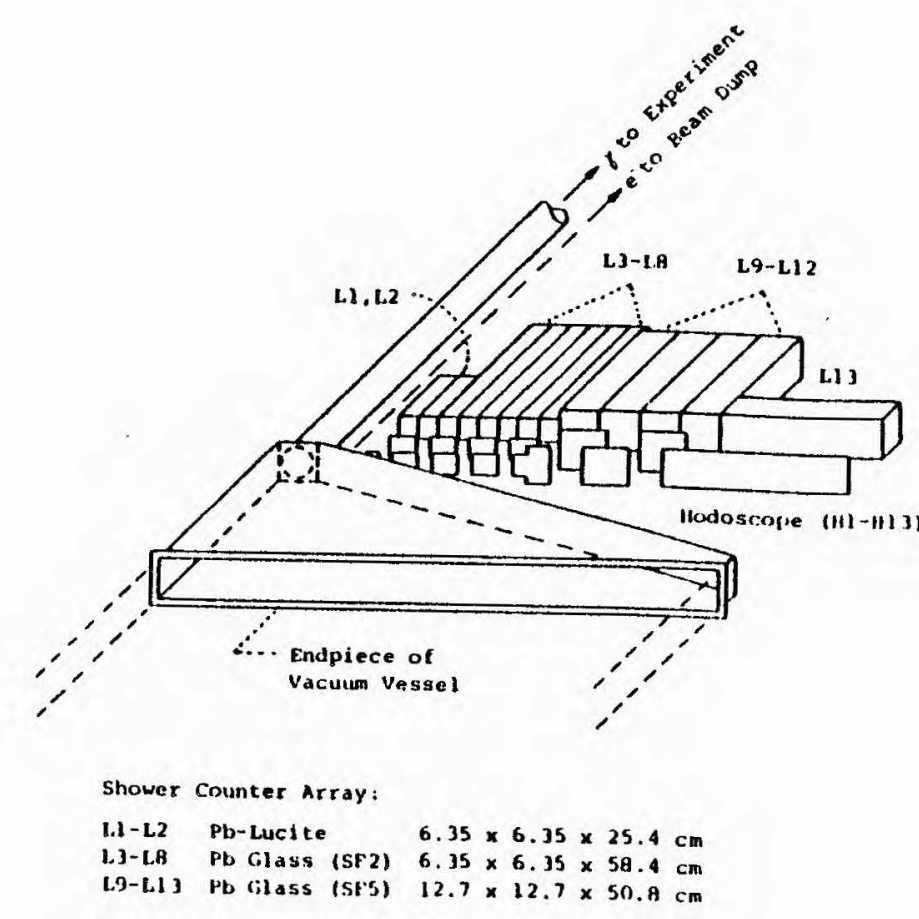

1
$\stackrel{5}{5}$
1

Figure 2-4 The tagging counter system. 
ELECTRON BEAM YIELD

PER INCIDENT PROTON

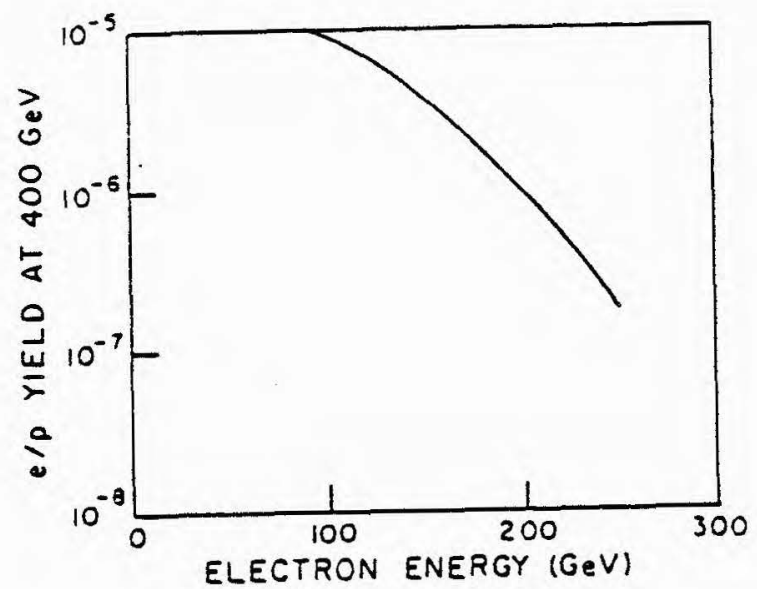

Figure 2-5 The fractional electron yield per incident proton as a function of the electron beam energy.

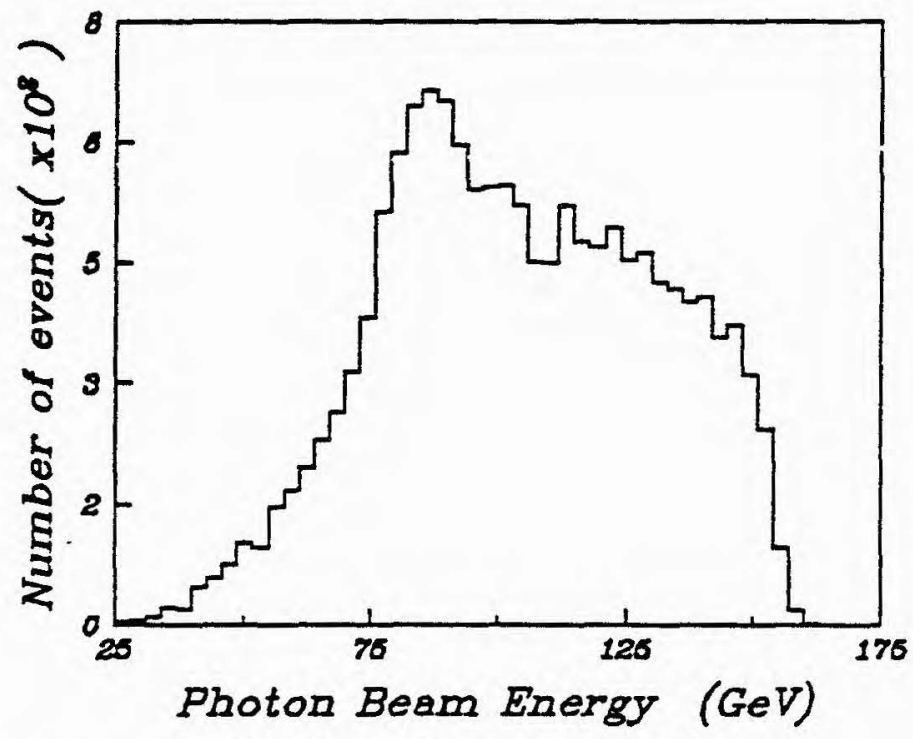

Figure 2-6 The energy spectrum of the photon beam. That portion above $75 \mathrm{GeV}$ is the true bremsstrahlung spectrum. Below it are those photons produced by multiple bremsstrahlung within the copper radiator. 
TAGGED PHOTON LABORATORY

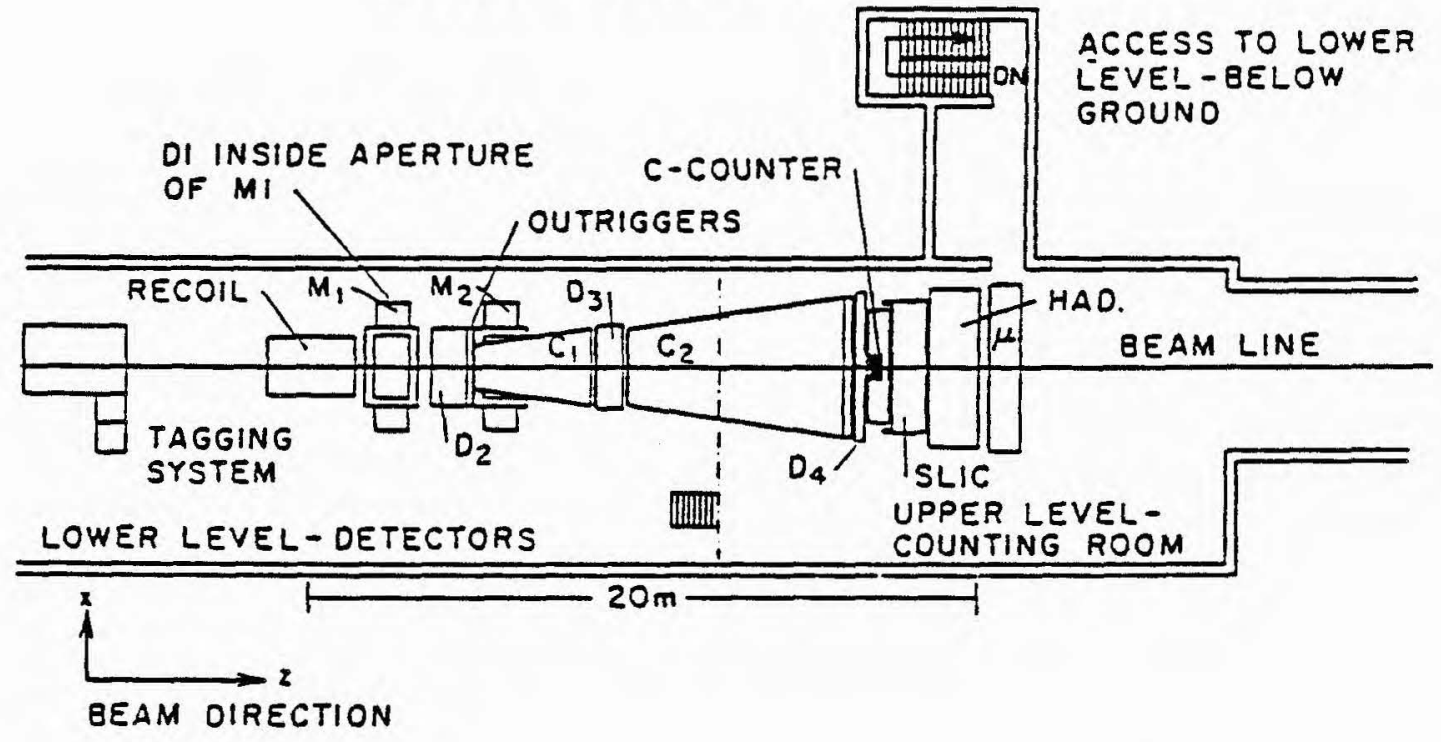

Figure 2-7 A layout of the tagged photon laboratory and the accompanying spectrometer.

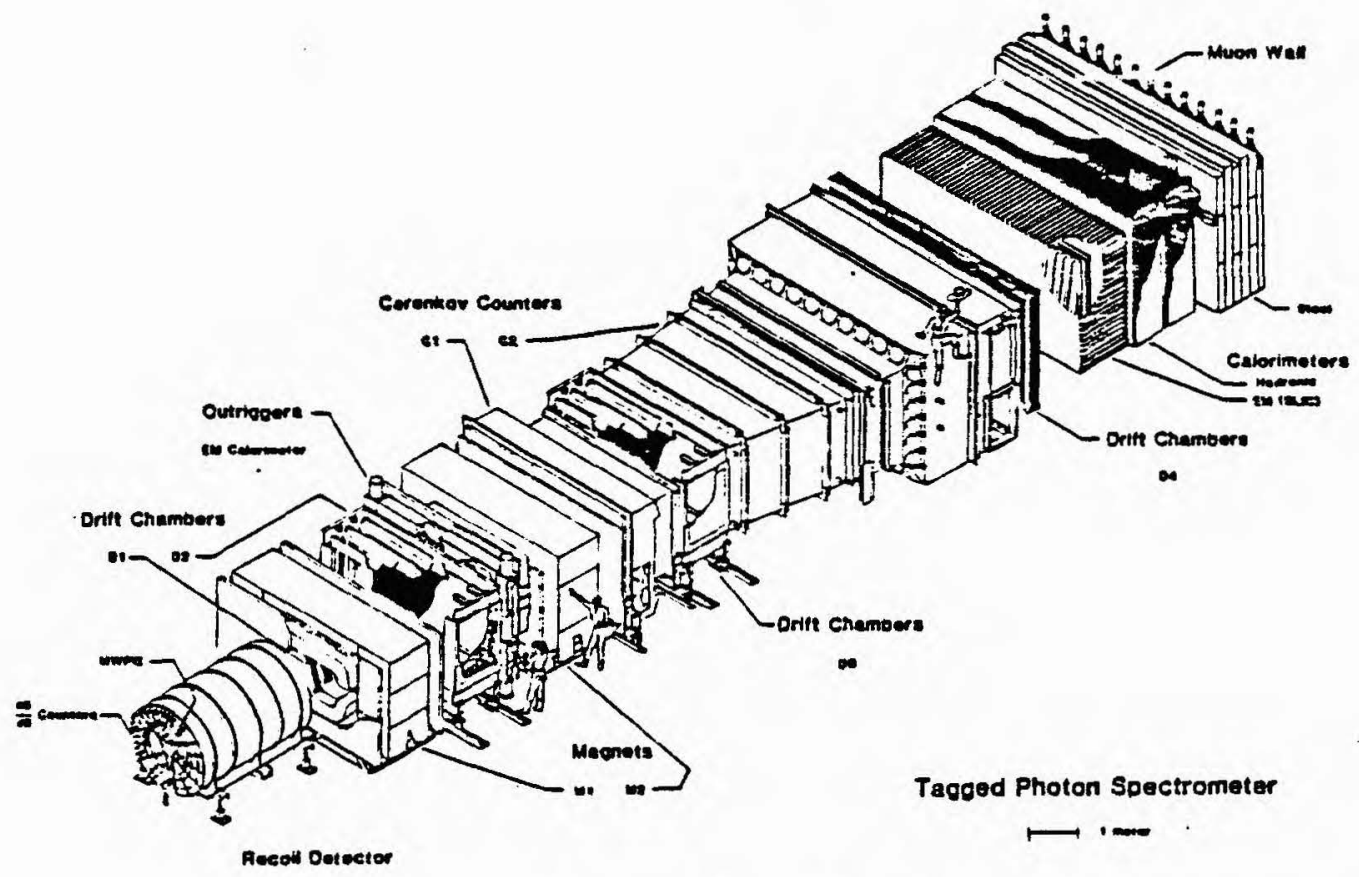

Figure 2-8 The Tagged Photon Spectrometer (TPS). 


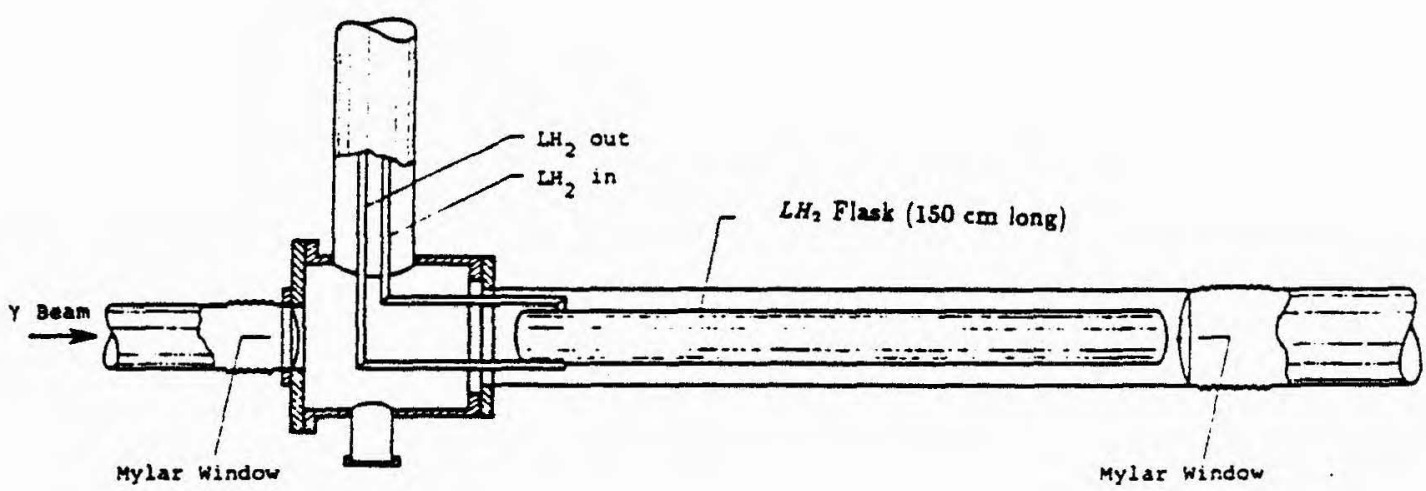

Figure 2-9 Schematic of the hydrogen target.

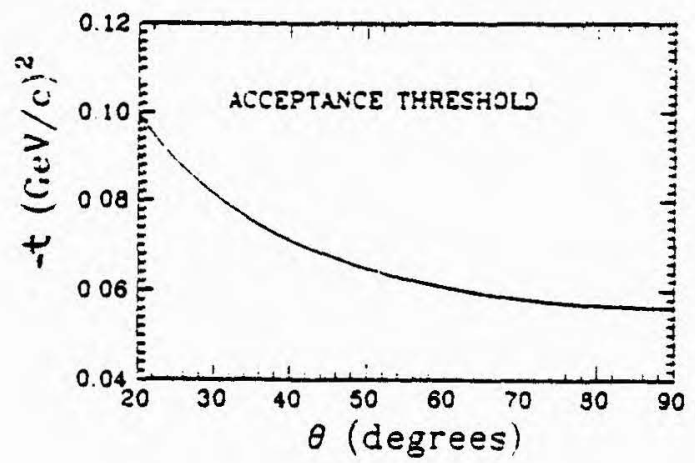

Figure 2-11 Acceptance threshold in momentum transfer $t$ versus the recoil polar angle $\theta$. 


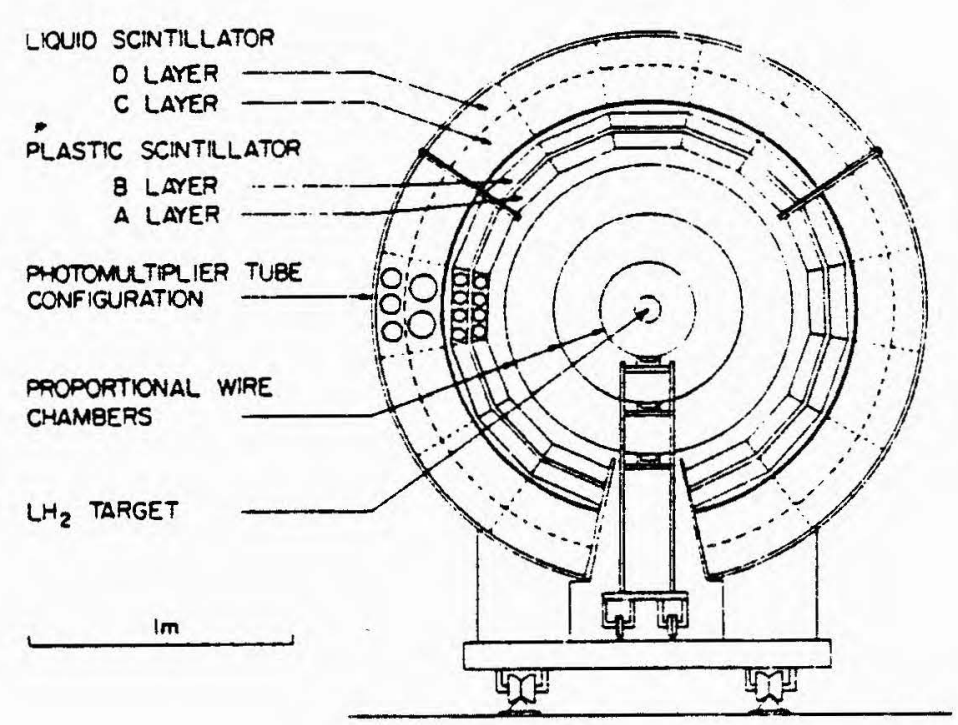

(a)

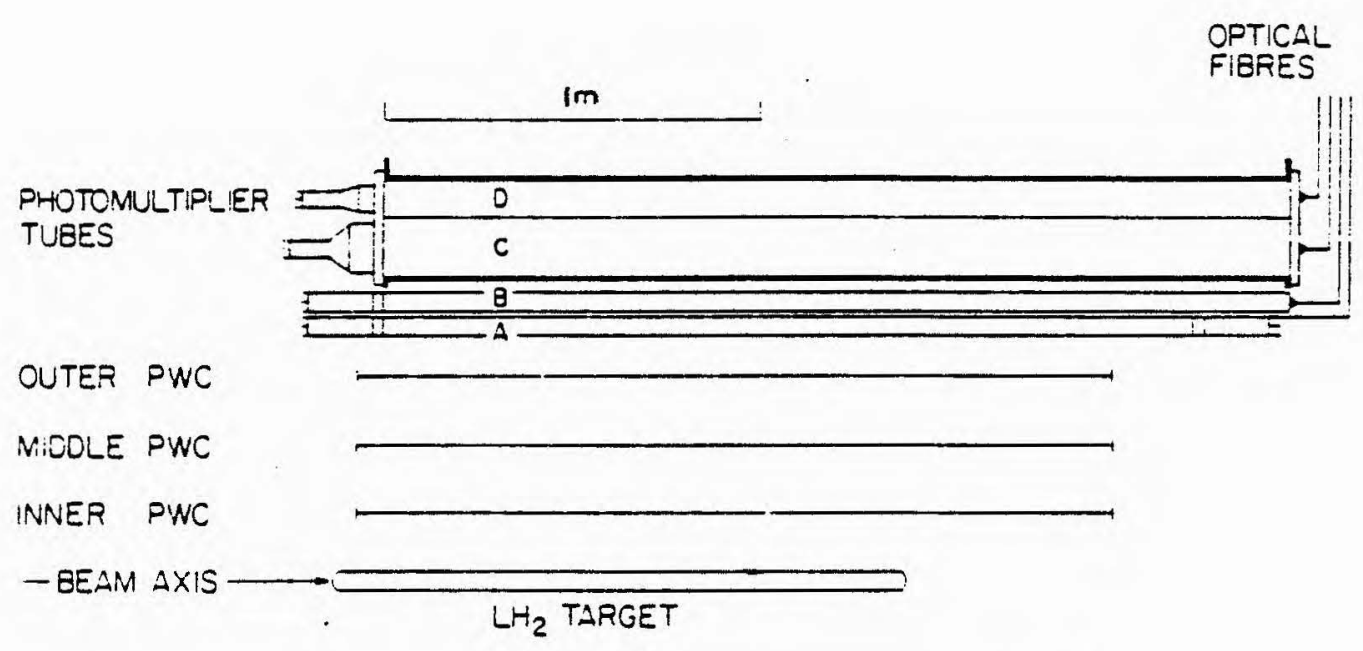

(b)

Figure 2-10 Two views of the Recoil Detector. Part (a) presents the front view while (b) displays a radial view. 


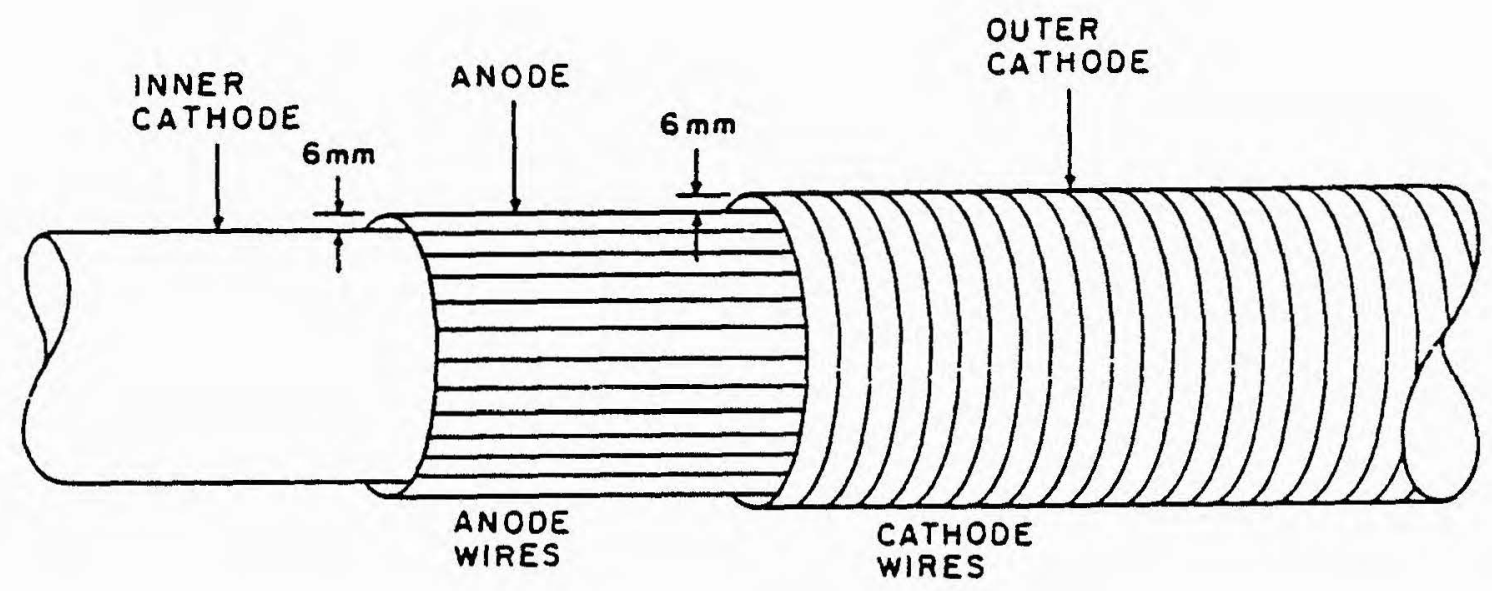

CUTAWAY VIEW OF PWC CHAMBER

(a)

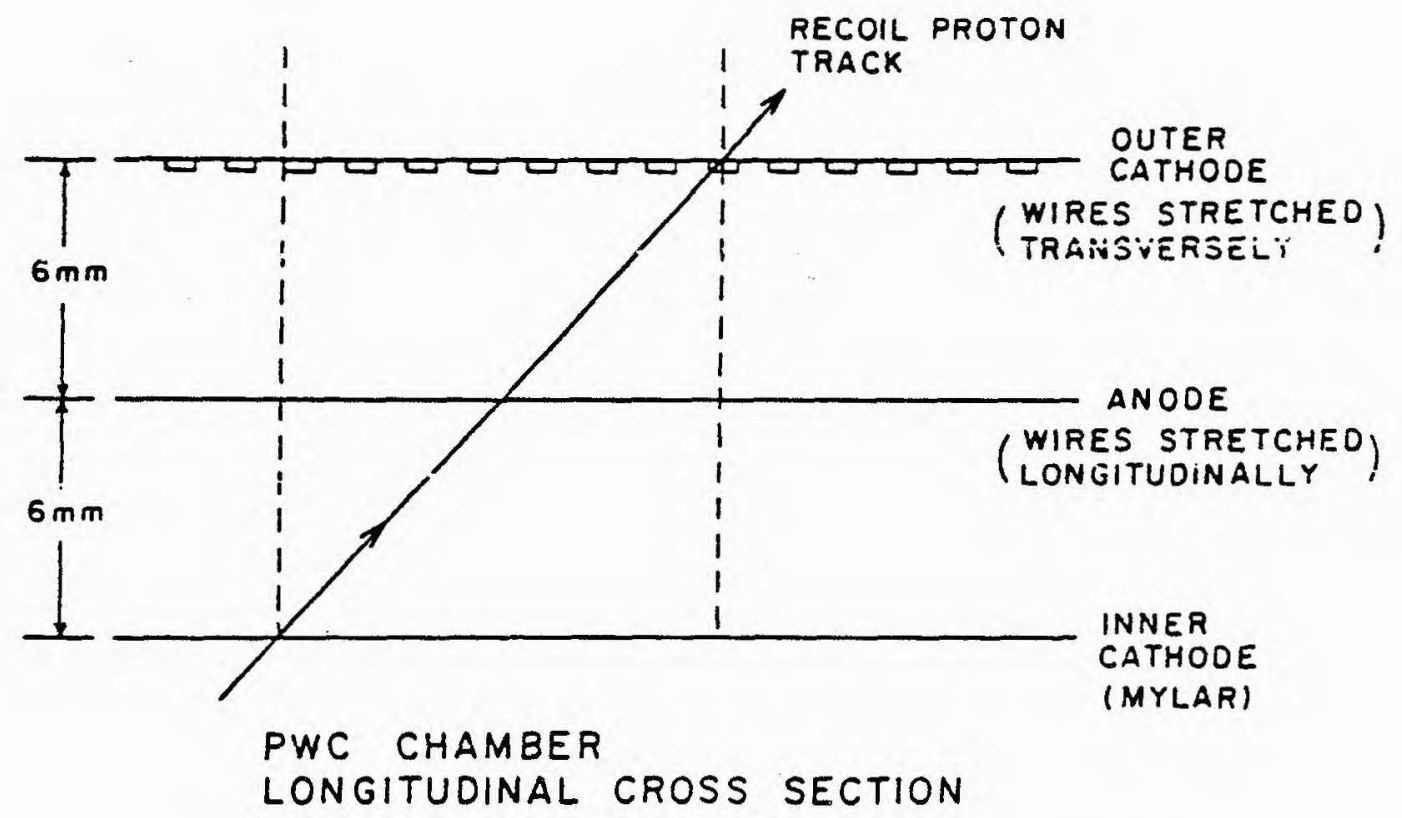

(b)

Figure 2-12 Two views of a Proportional Wire Chamber (PWC). 
DI ASSEMBLY

\section{DRIFT CHAMBER SYSTEM (TOP VIEW) (TOTAL OF 29 PLANES)}

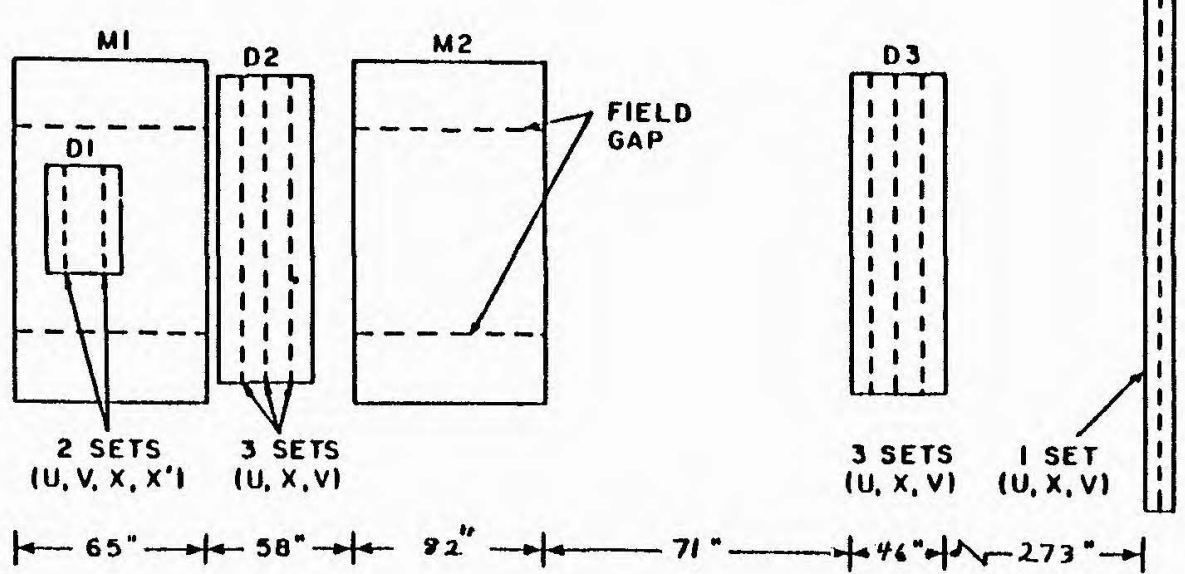

Figure 2-13 Layout of the Drift Clamber System, and the two magnets $\mathrm{M} 1$ and $\mathrm{M} 2$.

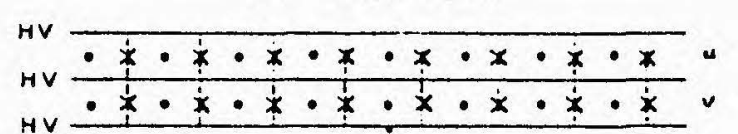

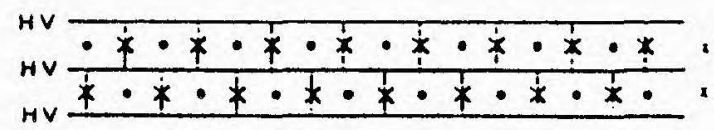

X - FIELO WIRES

- - sEnSE wiRes

hV - hIGH VOLTAGE FIELD PLANES

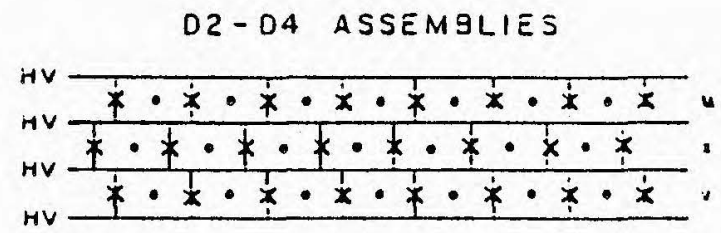

DRIFT CHAMBER CELL STRUCTURE

Figure 2-14 The cell structure of the Drift Chambers. 


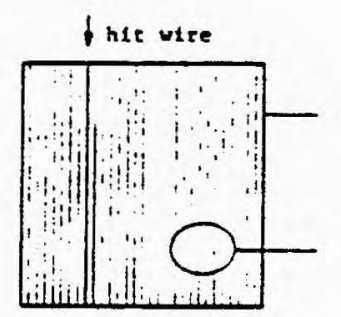

Scheastic of a MMPC with one hit wite.

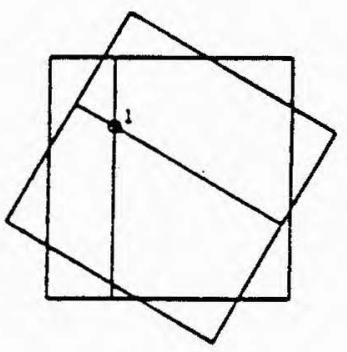

Two chambers, each with one hit wite:

Consistenc with one crack passing chrough the chambers at the incersection poine 1 .

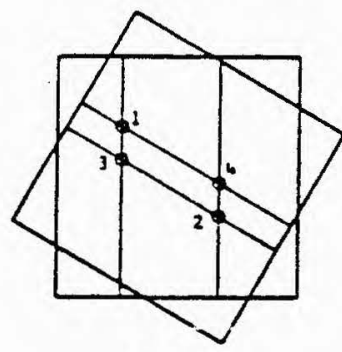

No chambers, each with two hit wires: Consistent with two tracks passing through the chabers. Tracks are at Incersection poincs 1 and 2 , or 3 and 4 (ambiguous!)

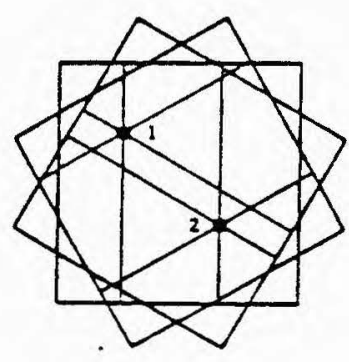

Three chambers, each with two hit wires: Consistent with two tracks passing through the chambers at the incersection points 1 and 2 (unambiguous!)

Figure 2-15 Schematic displaying the necessity of three different wire orientations for proper track reconstruction. 

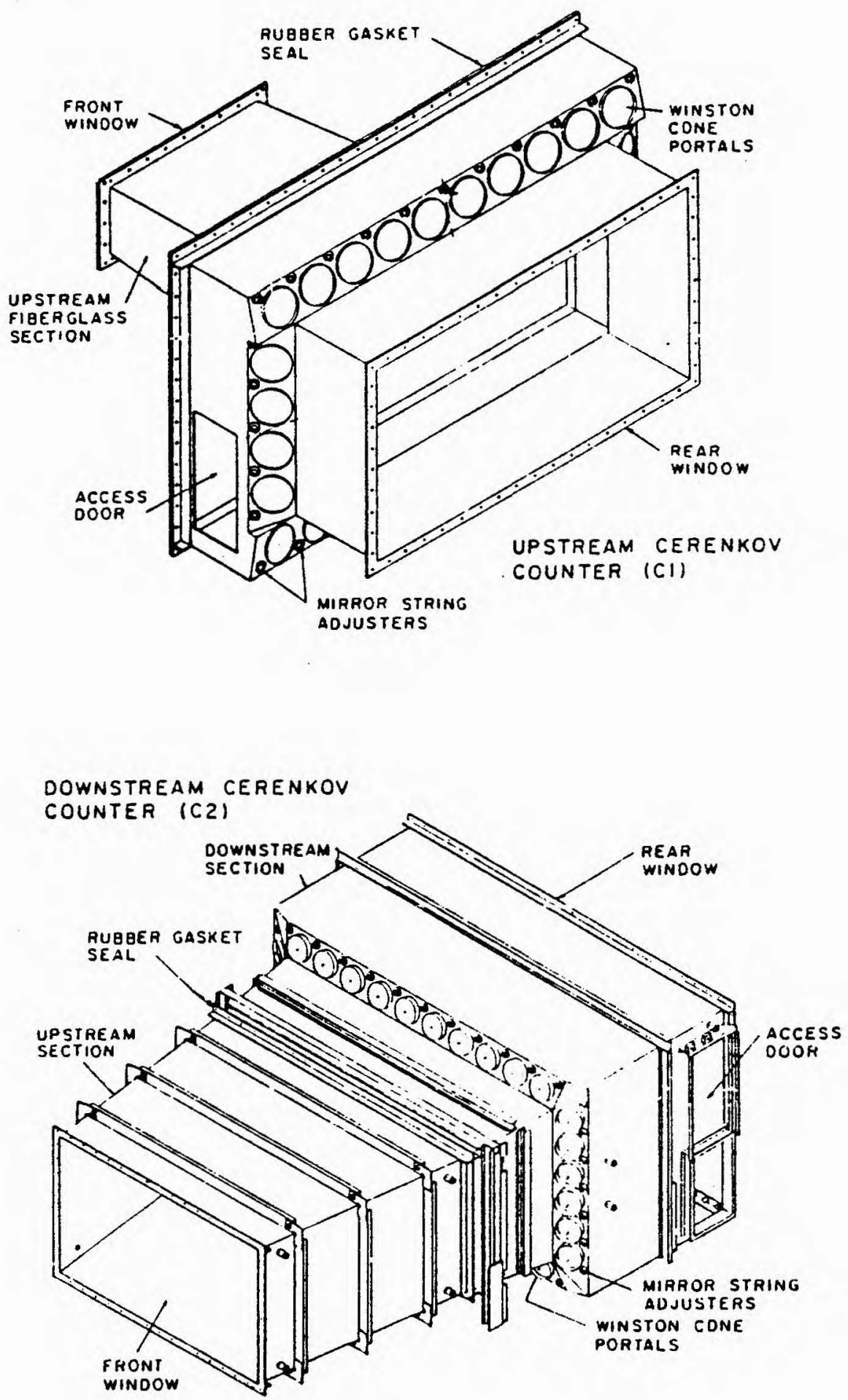

Figure 2-16 Schematic of the two Cerenkov counters. 


\section{CERENKOV MIRROR SEGMENTATION}

\begin{tabular}{|c|c|c|c|c|}
\hline i & \multicolumn{2}{|c|}{4} & 5 & $\begin{array}{l}\mathbf{T} \\
\vdots \\
\vdots \\
\vdots\end{array}$ \\
\hline 1 & 1 & 2 & 3 & i \\
\hline I & & & & i \\
\hline 1 & & & & $\begin{array}{l}\text { i } \\
\text { i }\end{array}$ \\
\hline
\end{tabular}

\section{CERENKOV MIRROR SUSPENSION}

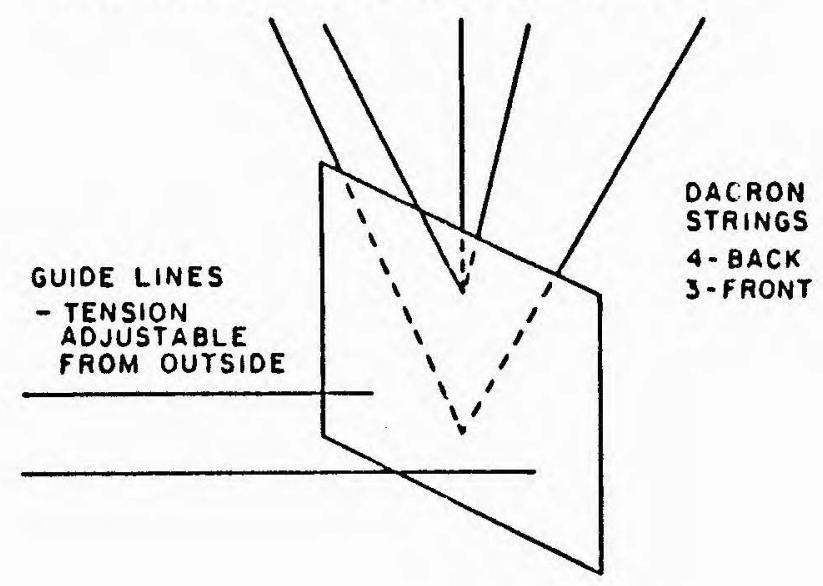

Figure 2-17 Cerenkov counter mirror segmentatior. and support scheme.

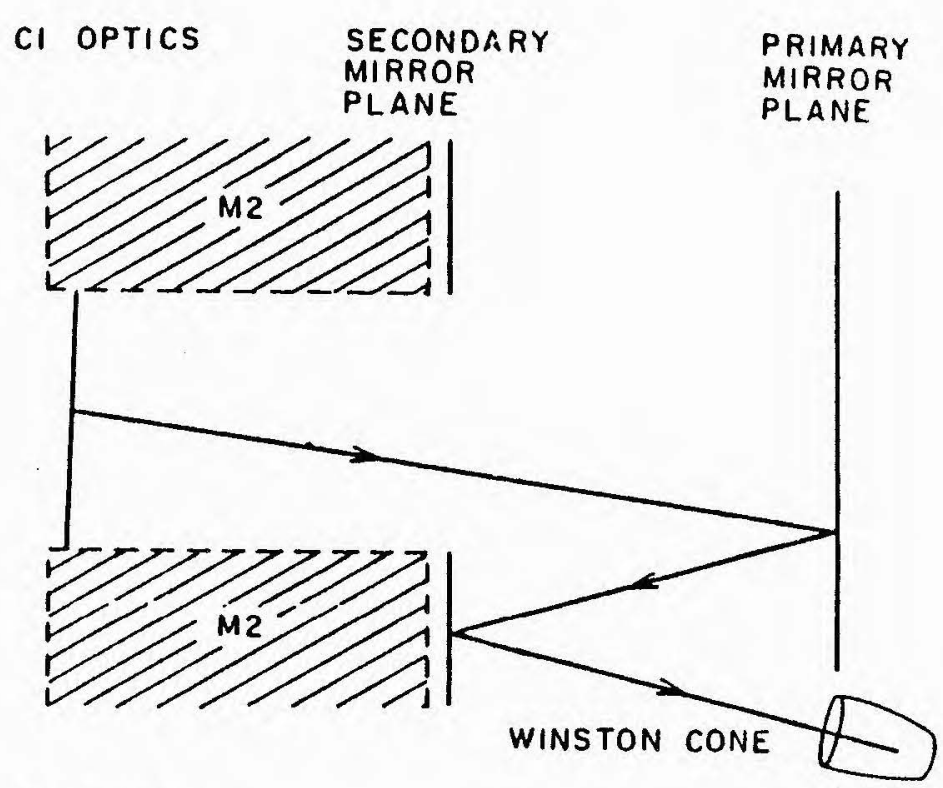

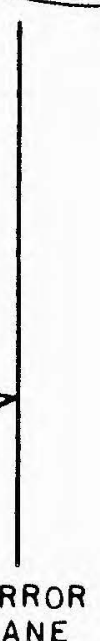

Figure 2-18 Cerenkov counter optics. 


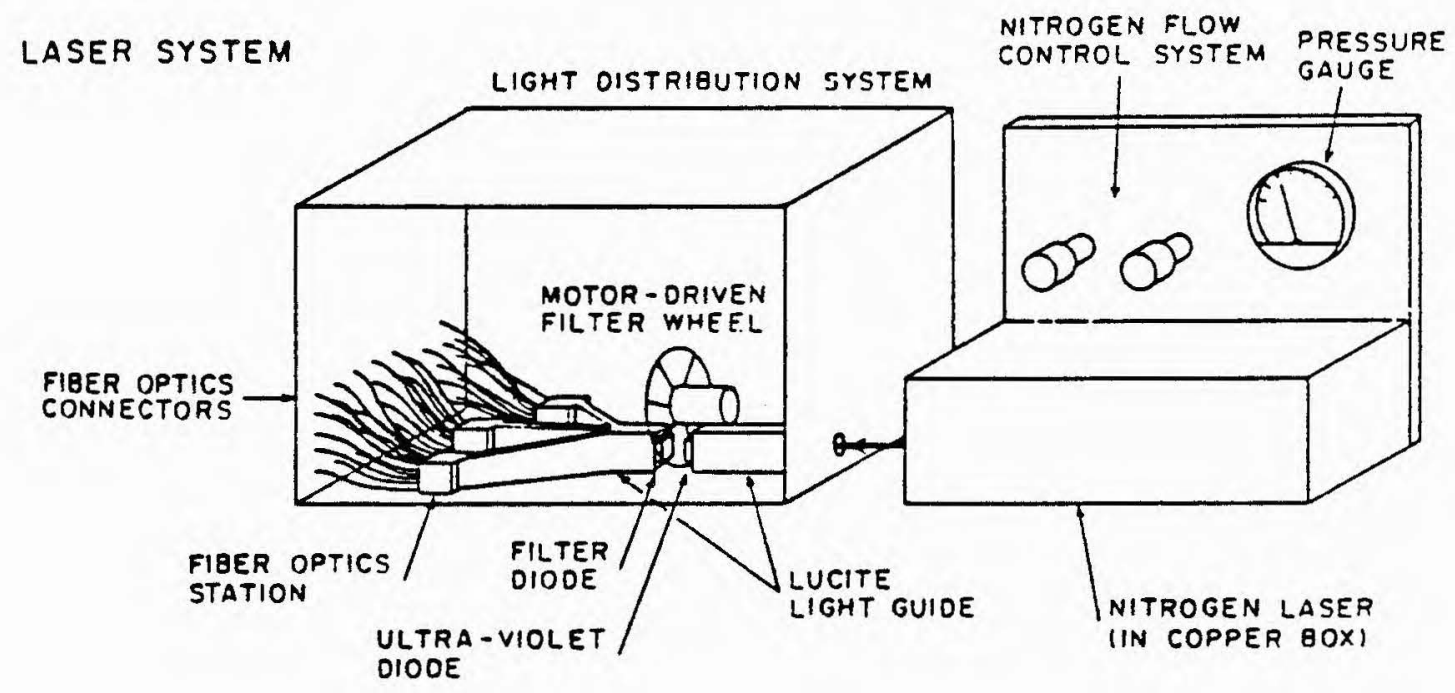

Figure 2-19 The laser system used for monitoring the phototube gains.

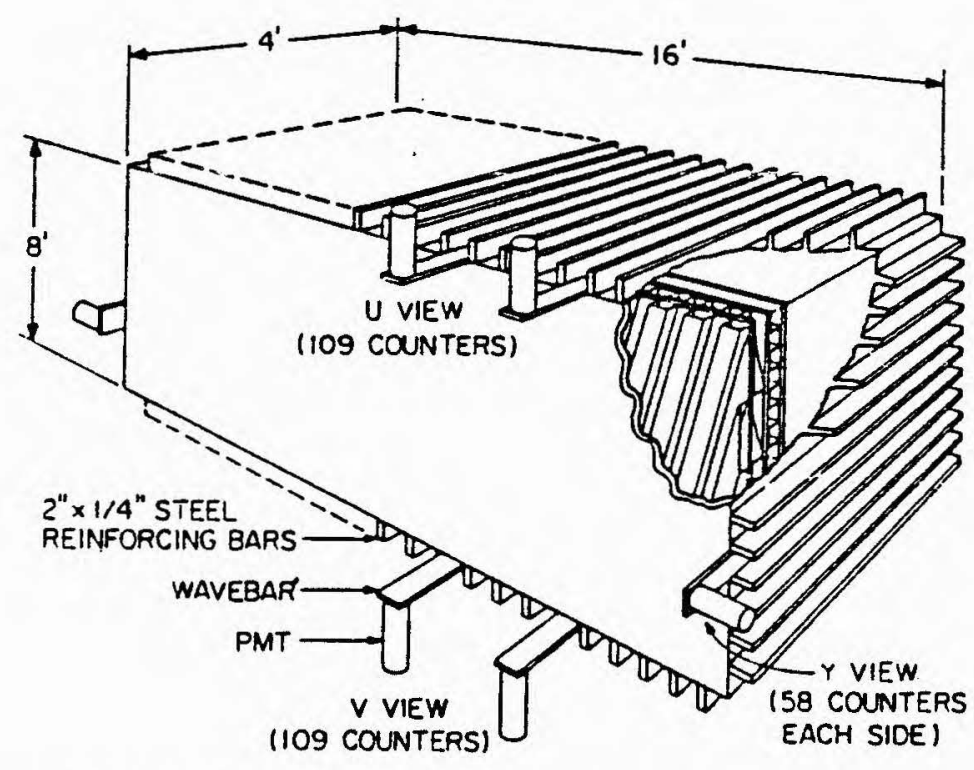

LEAD/LIOUID SCINTILLATOR SHOWER COUNTER (SLIC) (SCHEMATIC)

Figure 2-20 Schematic of the SLIC. 


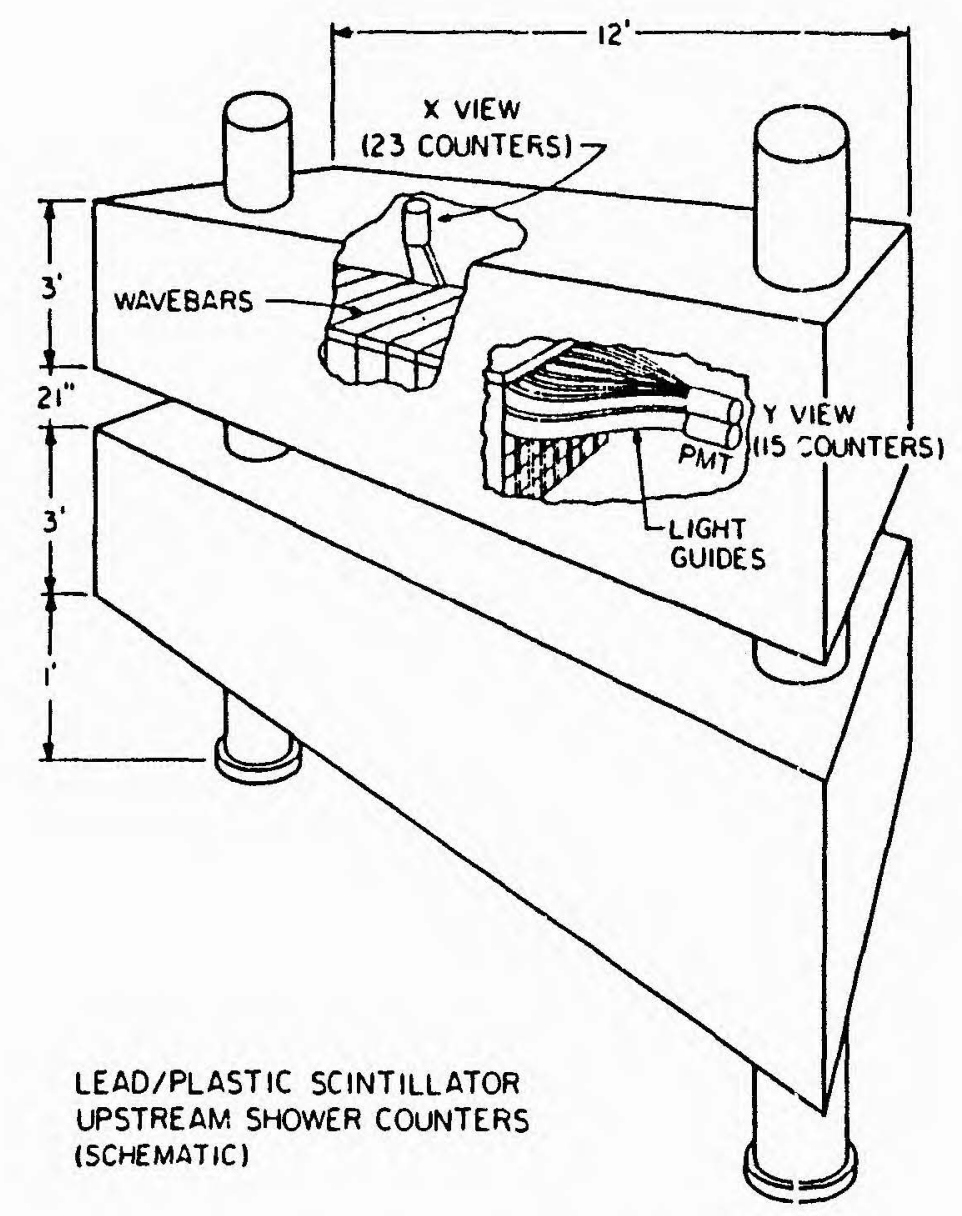

Figure 2-21 Schematic of the Outriggers

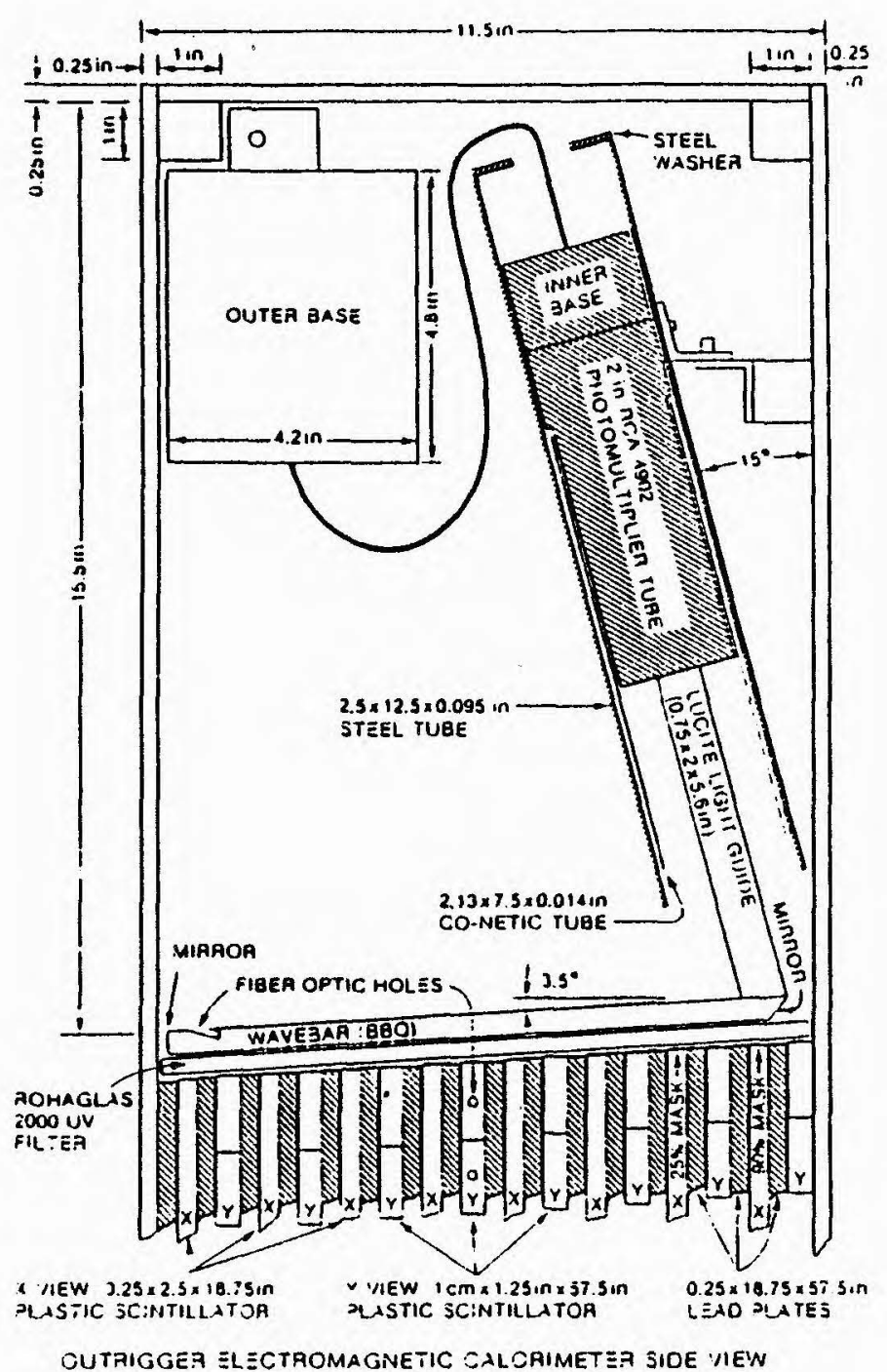

1
$\stackrel{\omega}{\infty}$
1

Figure 2-22 Side view of an Outrigger. 


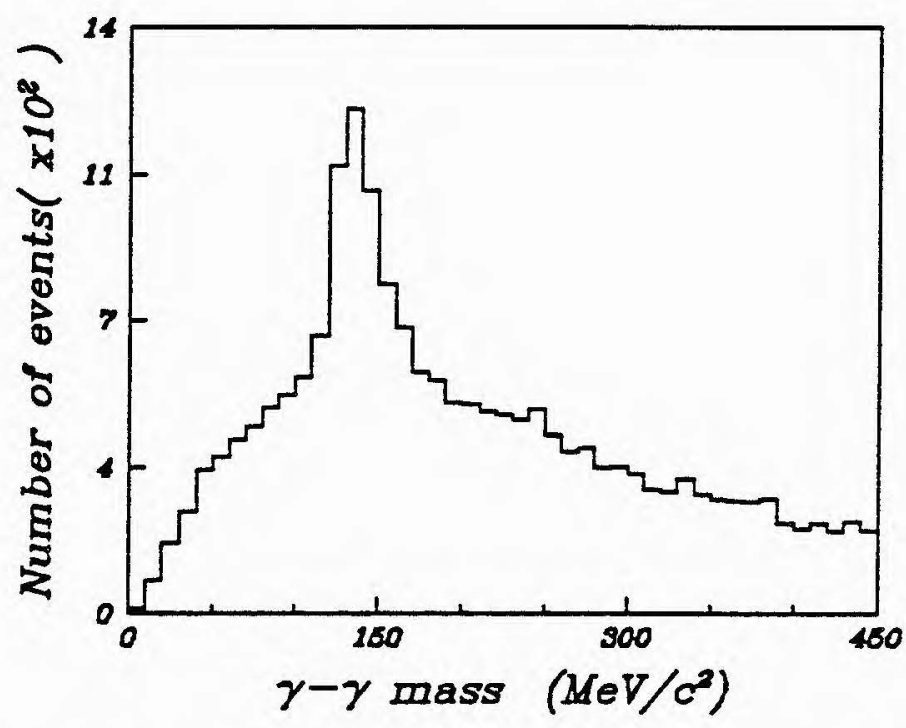

Figure 2-23 The invariant mass of pairs of reconstructed photons that showered within the SLIC detector. Note especially the $\pi^{0}$ peak above the combinatorial background.

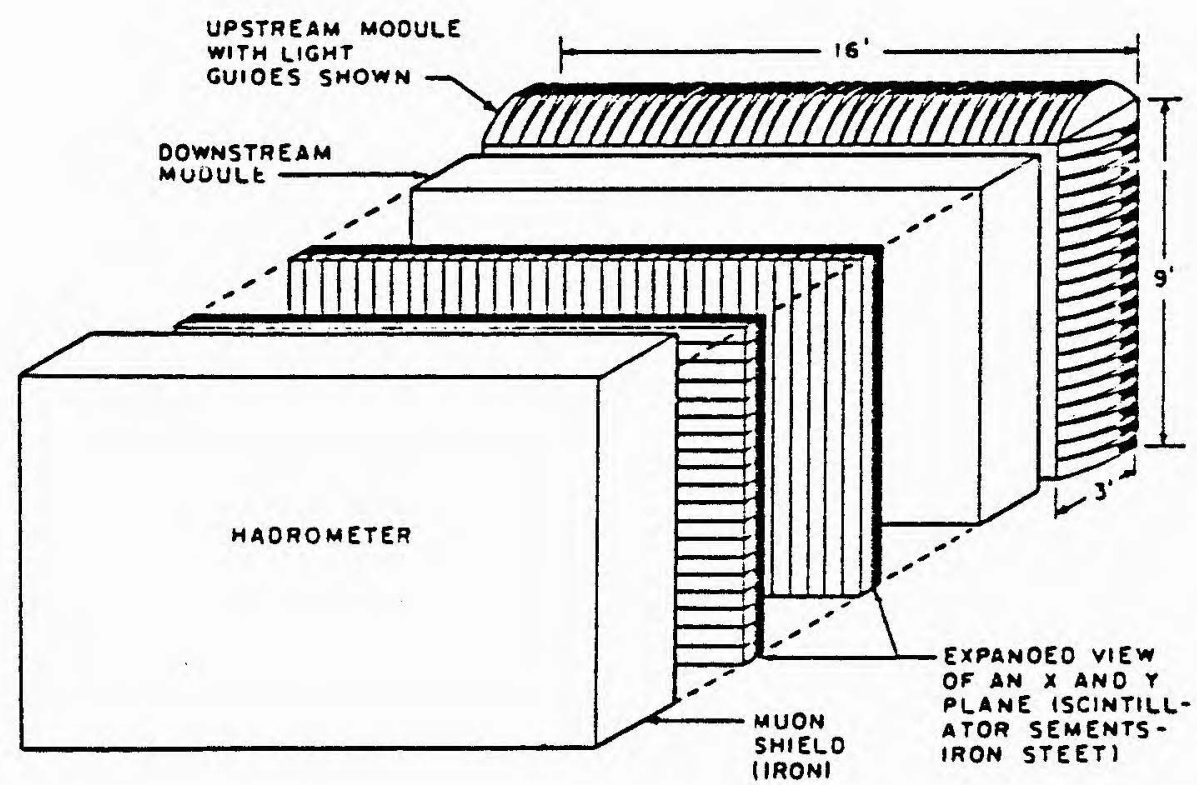

Figure 2-24 An expanded view of the Hadrometer. 


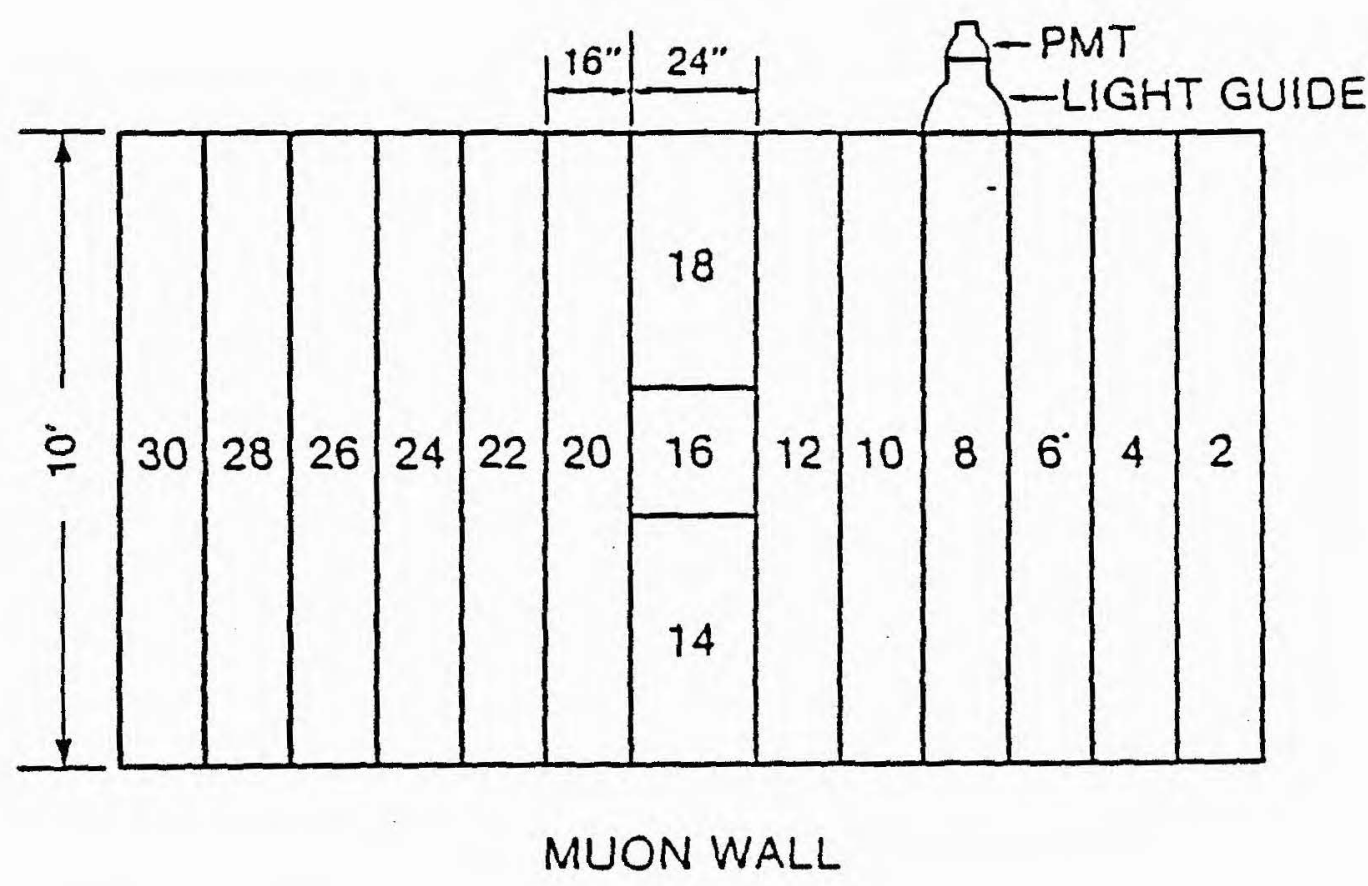

Figure 2-25 Layout of the Muon Wall.

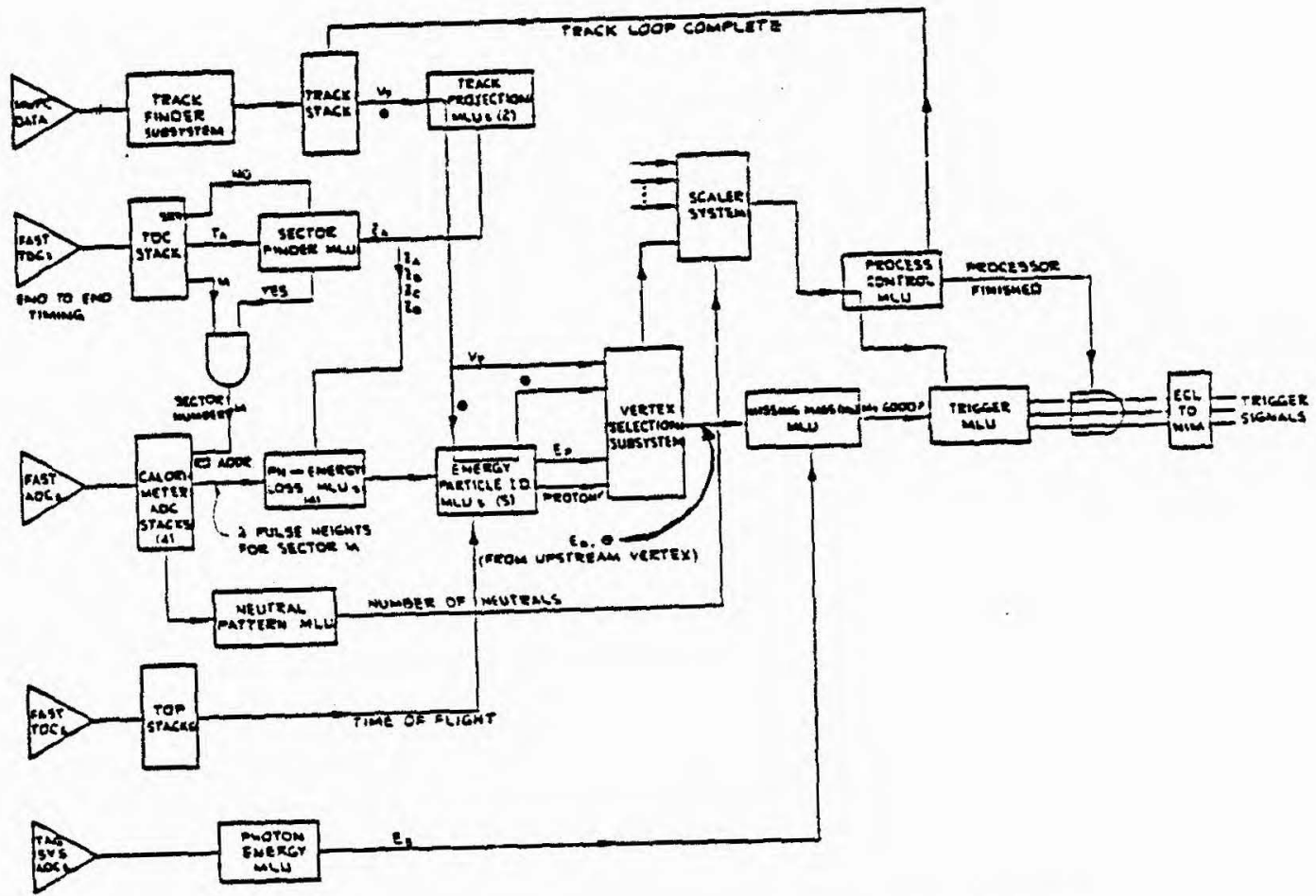

Figure 2-26 Schematic of the reconstruction process within the Trigger Processor. 

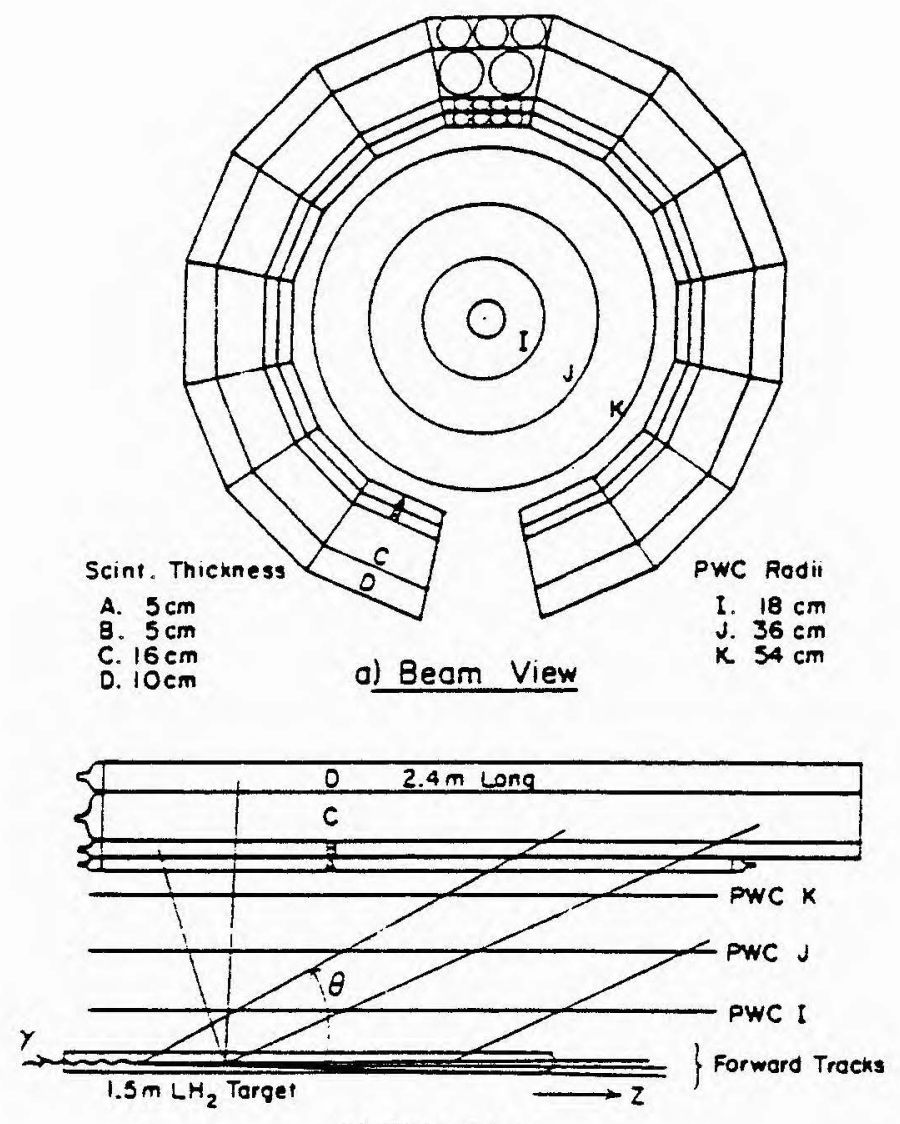

b) Side View

Figure 2-27 Layout of the Recoil Detector as viewed with respect to the Trigger Processor. 


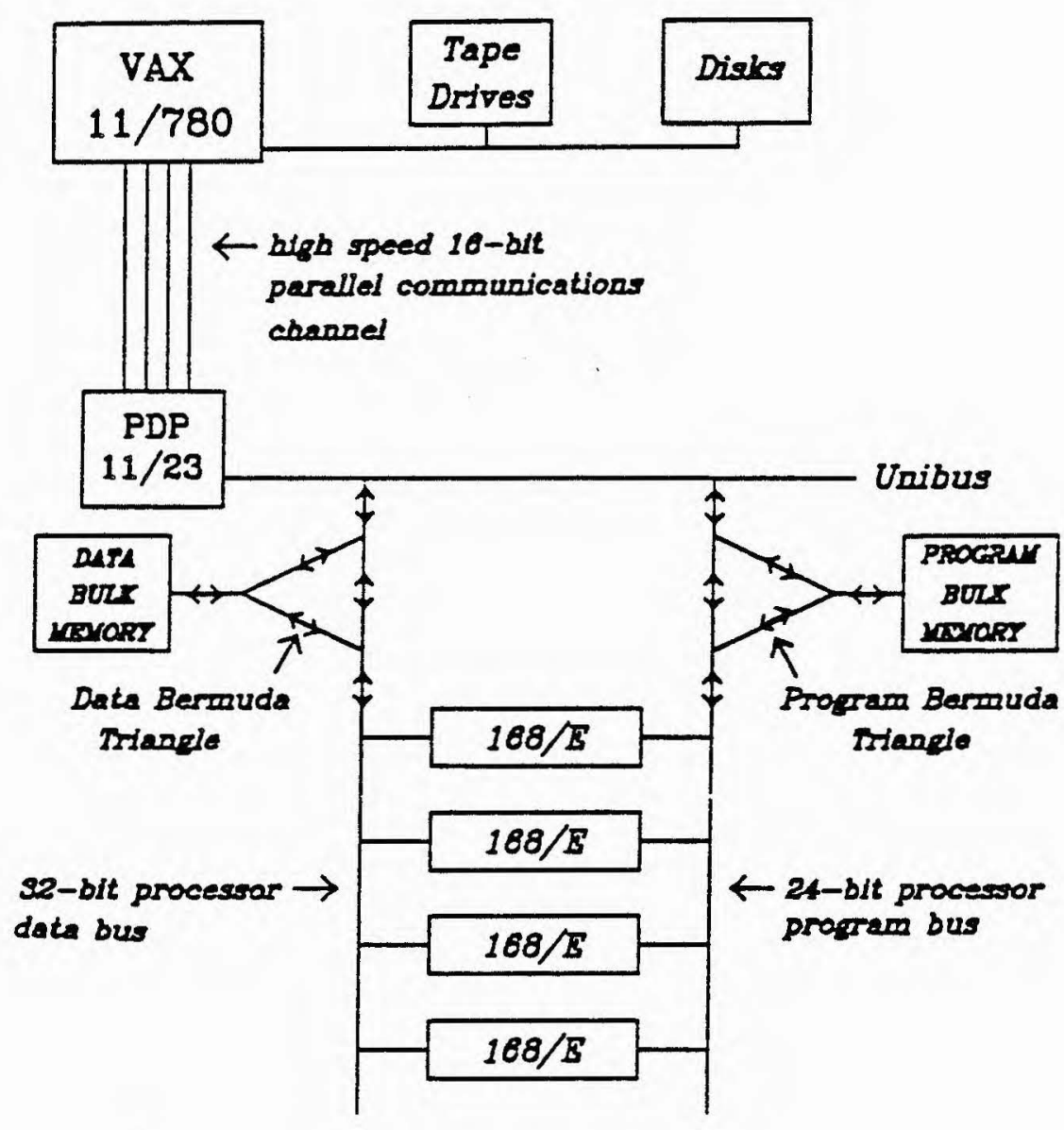

Figure 2-28 Simplified block diagram layout of the 168/E emulator system. 


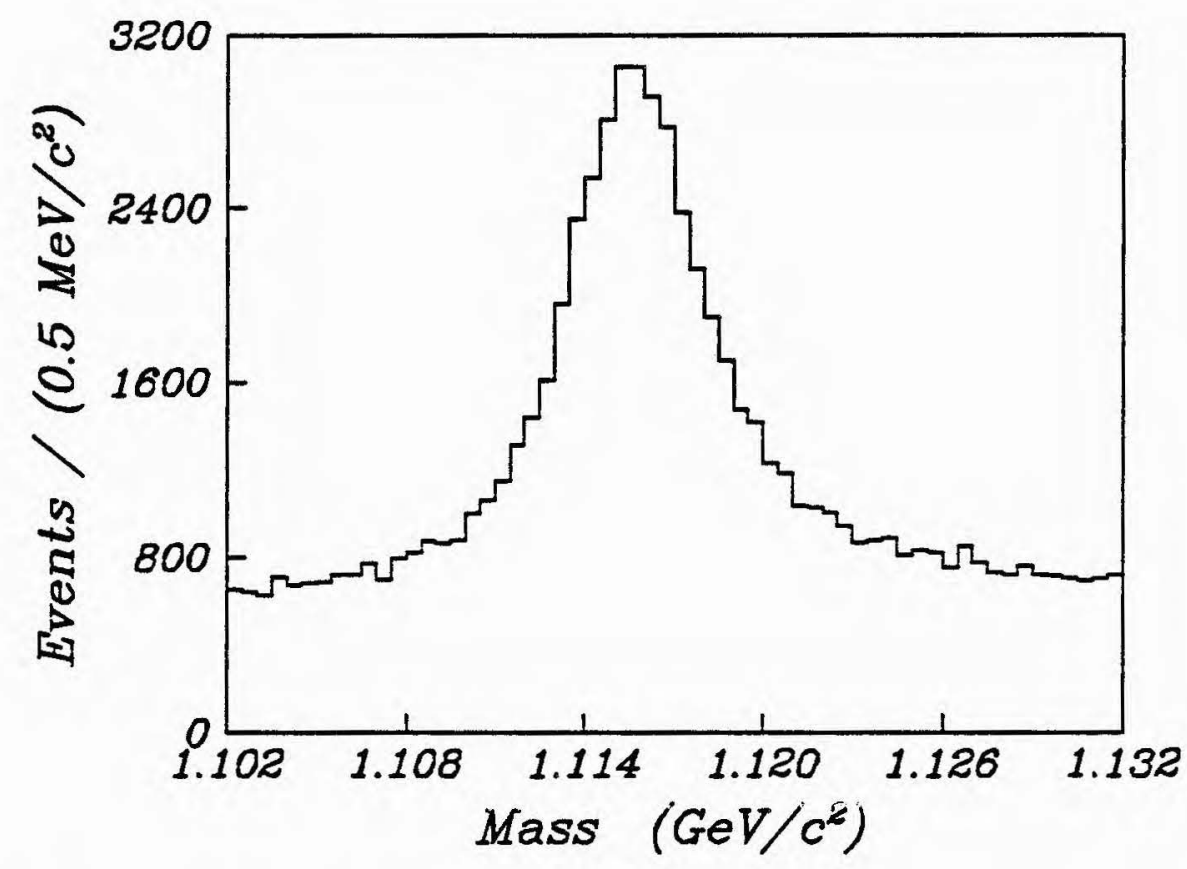

Figure 3-1 The $(\Lambda / \bar{\Lambda})$ signal obtained after completion of the background reduction in the $170 \mathrm{GeV}$ sample. Section (3-2) details the process of background reduction. 


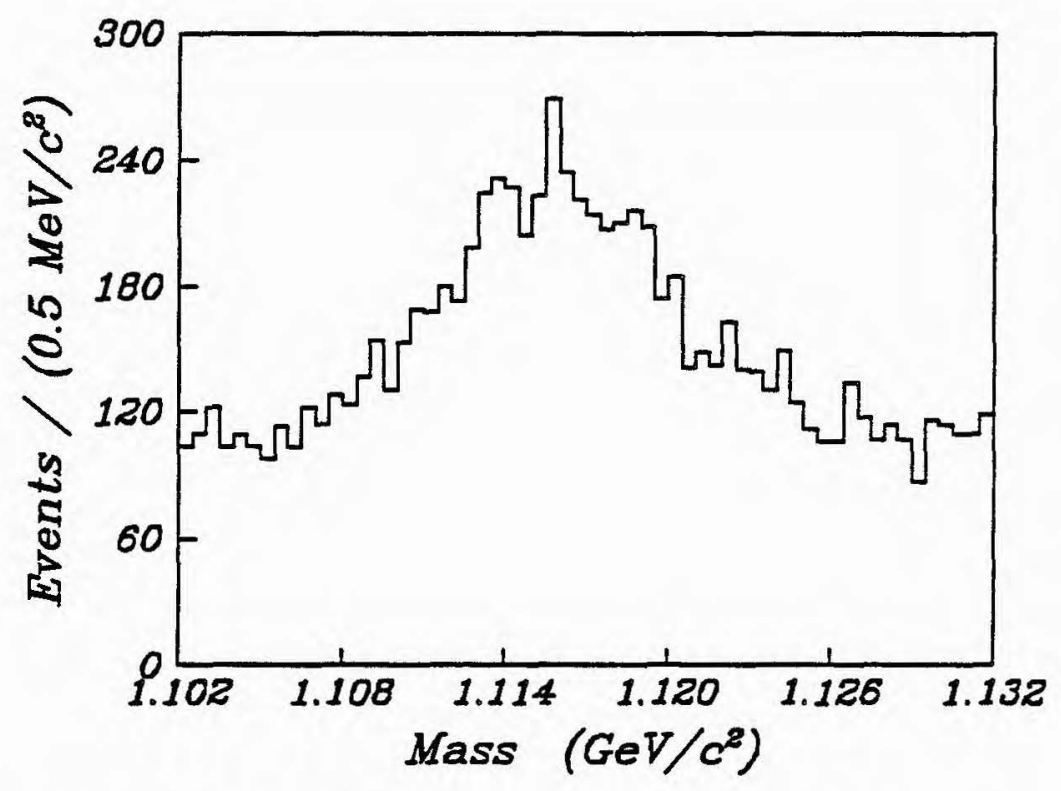

Figure 3-2(a) The $\Delta$ signal when both the proton and pion tracks traverse only the first two drift chambers.

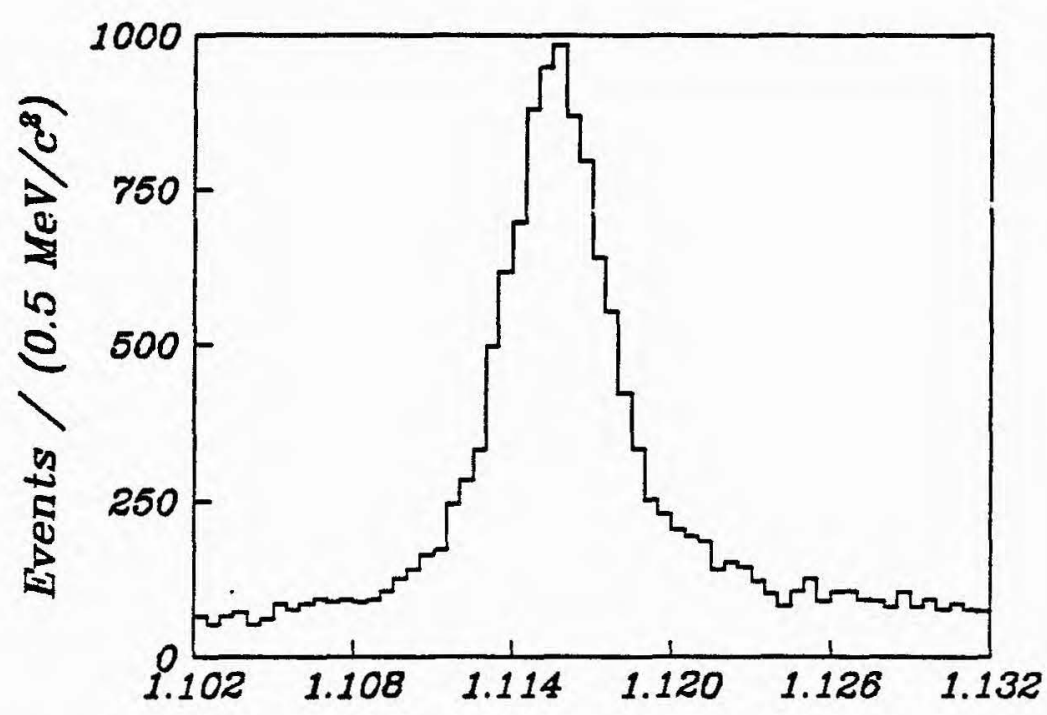

Figure 3-2(b) The $\Lambda$ signal when both decay tracks traverse three or four drift chambers. 


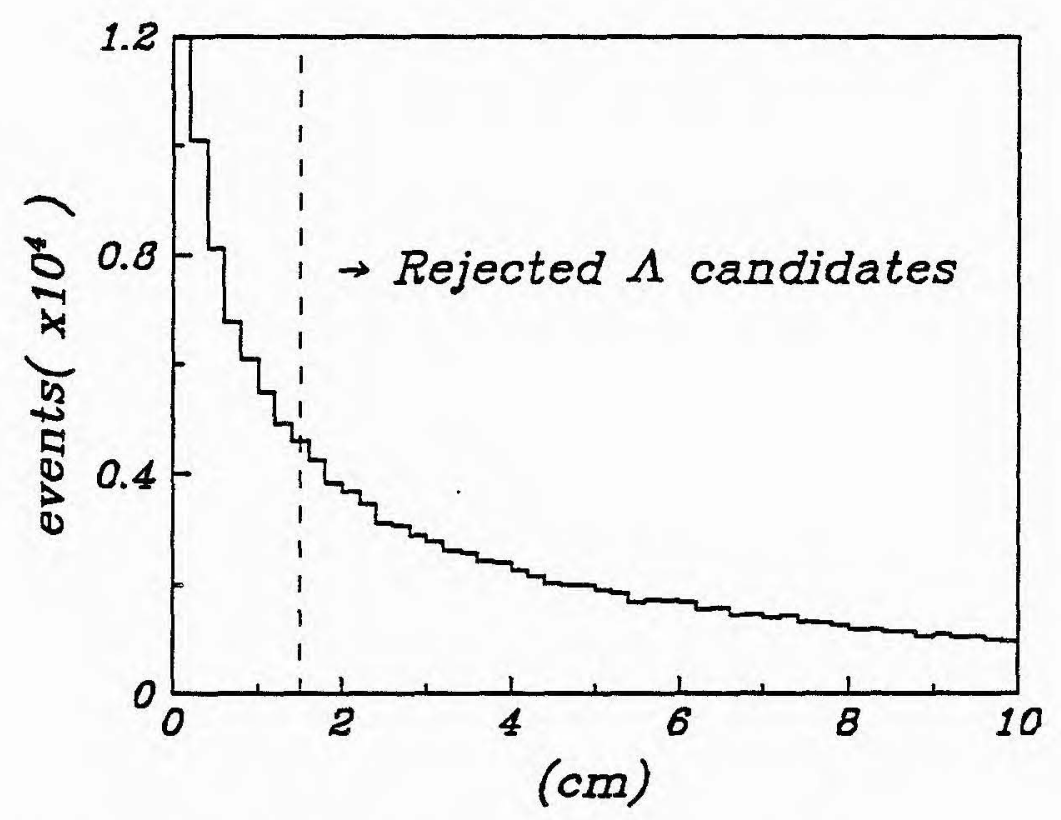

Figure 3-3(a) $D C A(p, \pi)$ for poor pions.

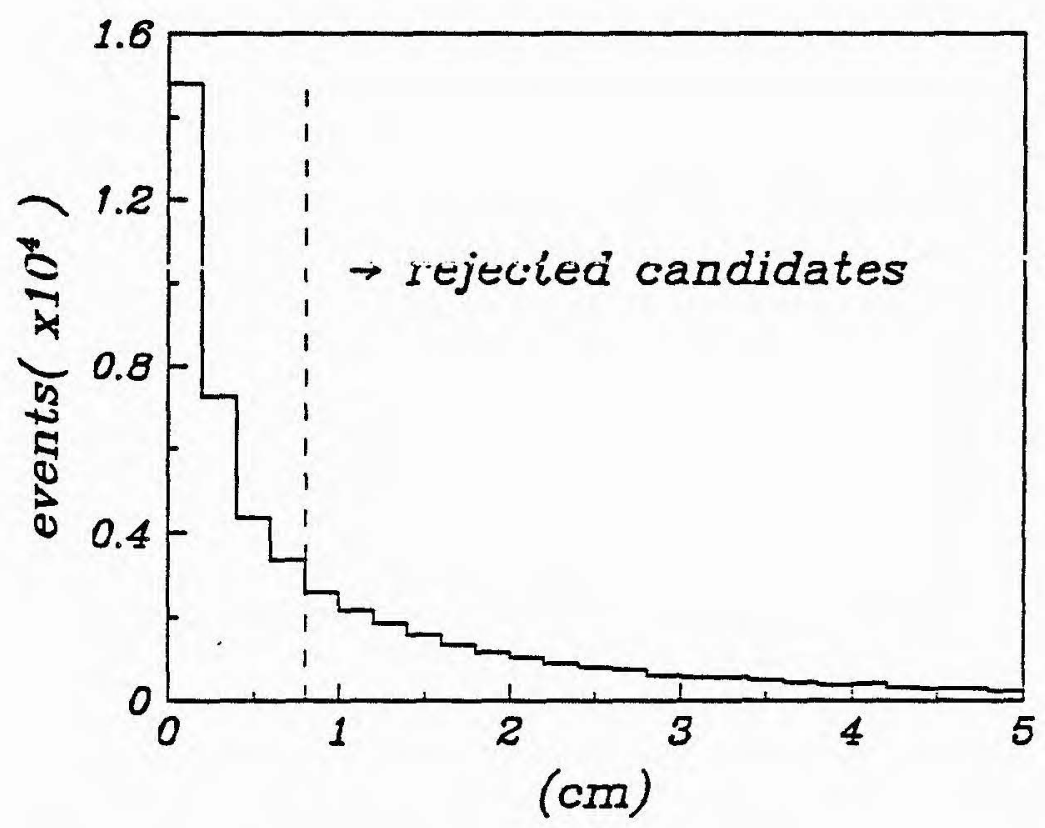

Figure 3-3(b) $D C A(p, \pi)$ for good pions. 


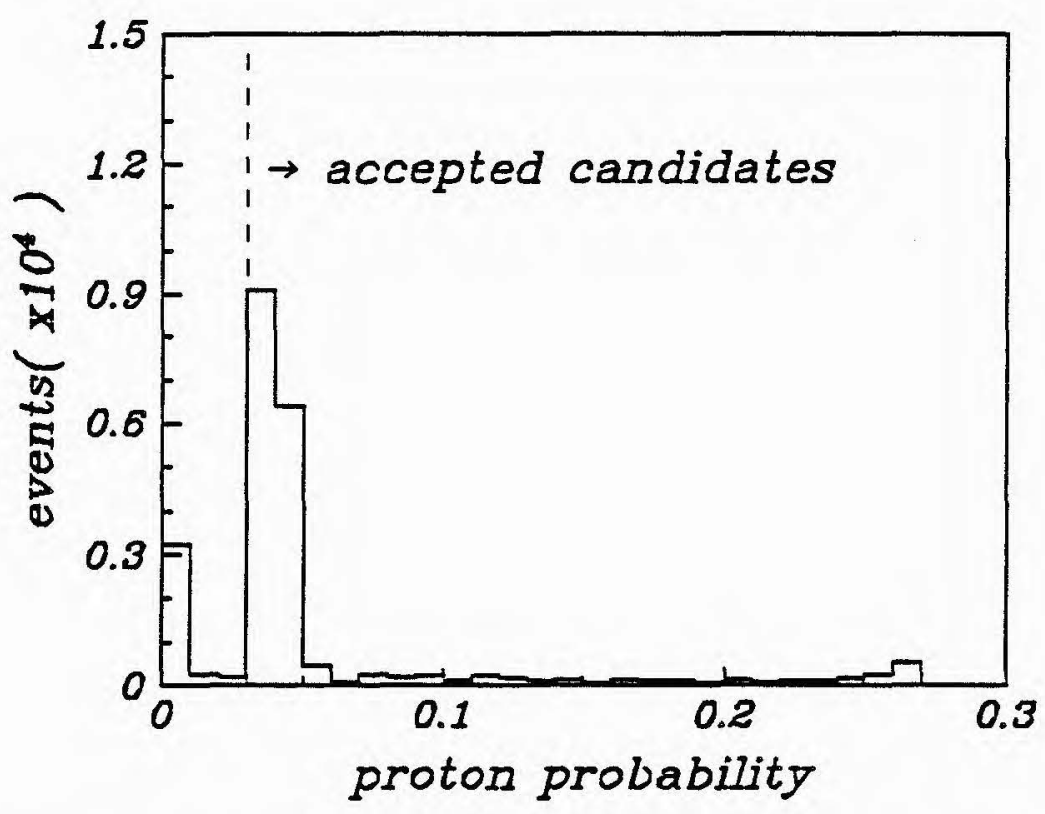

Figure 3-4(a) Proton identification probability for poor proton tracks.

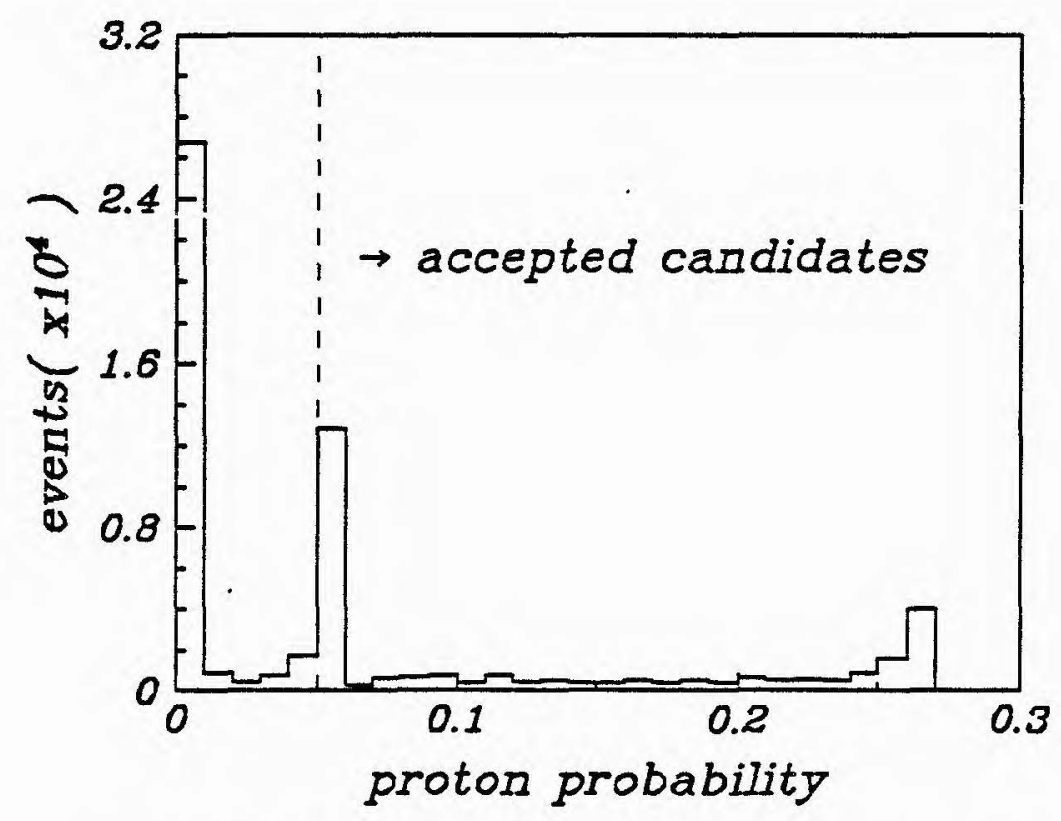

Figure 3-4(b) Proton identification probability for good proton tracks. 


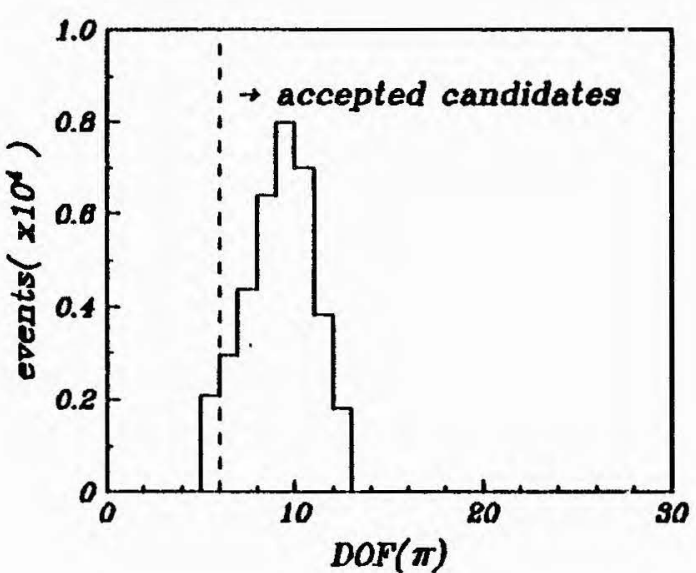

Figure 3-5(a) Degrees-of-freedom for the pion tracks. Figure (a) displays the distribution for category 3 (poor) tracks while figures (b) and (c) display the category $7 \& 15$ (good) tracks respectively.

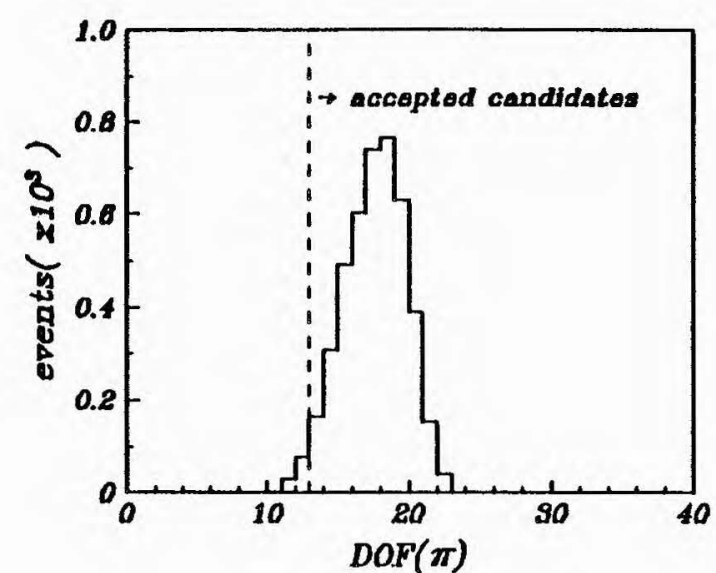

Figure 3-5(b)

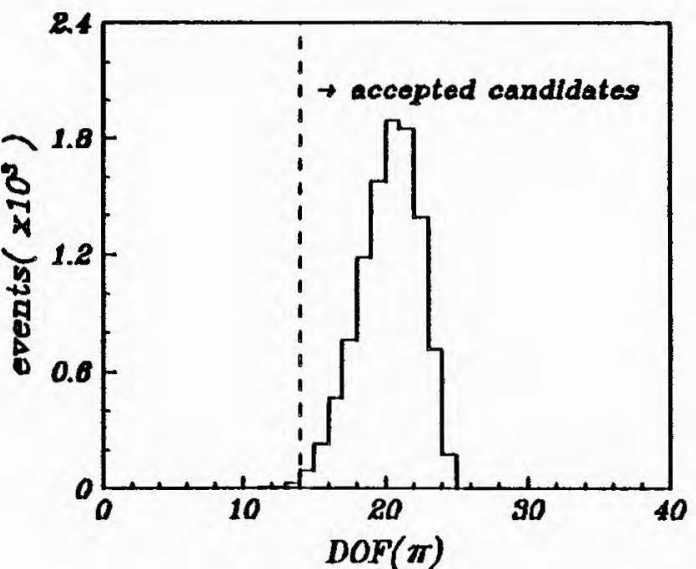

Figure 3-5(c) 


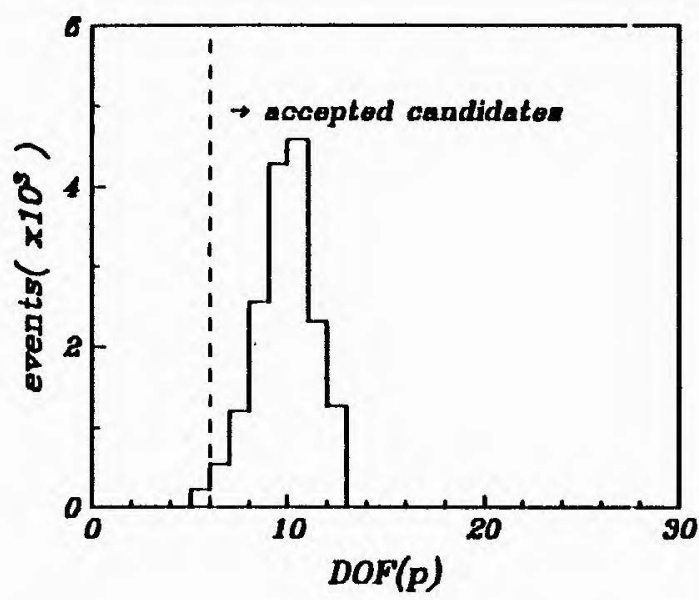

Figure 3-6(a) Degrees-of-freedom for the proton tracks. Figure (a) displays the category 3 tracks while figures (b) and (c) display the category $7 \& 15$ tracks respectively.

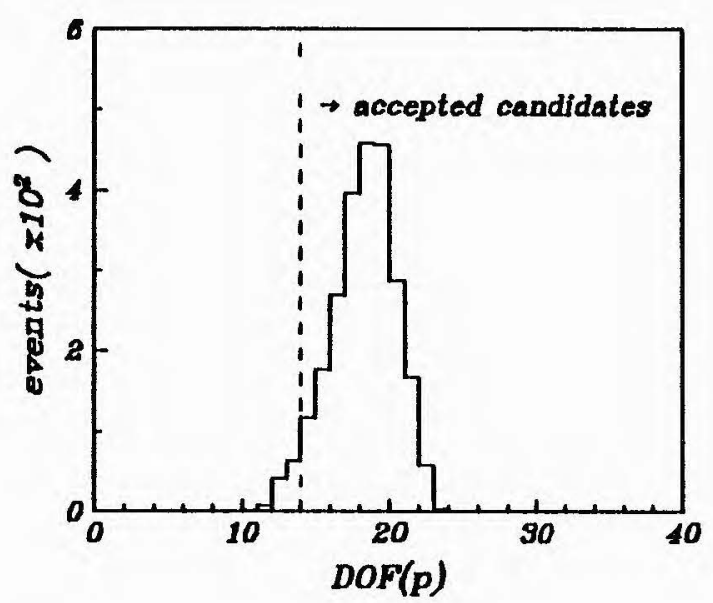

Figure 3-6(b)

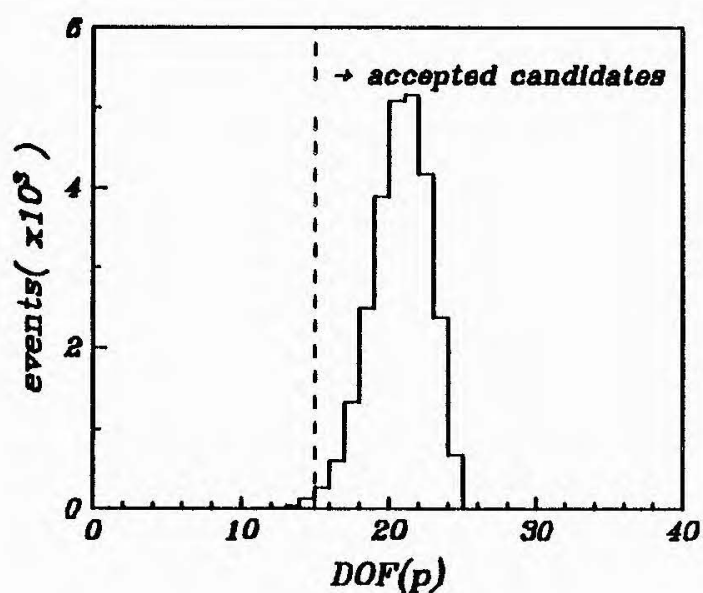

Figure 3-6(c) 


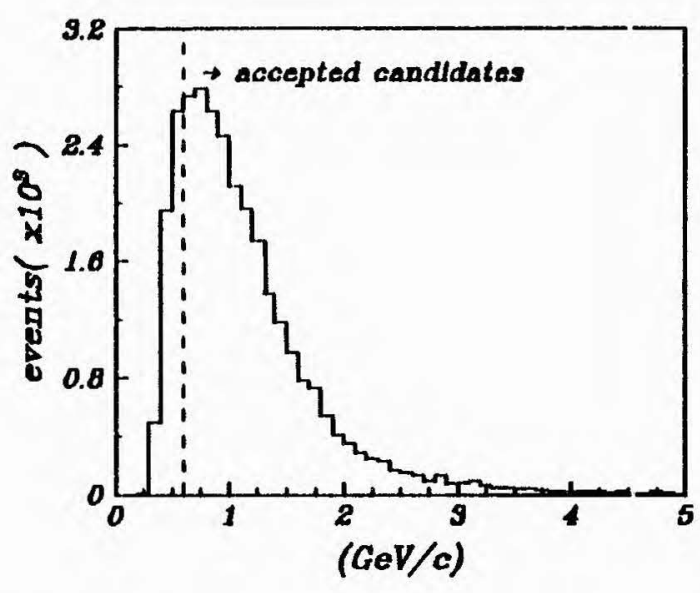

Figure 3-7(a) Figure (a) is the momentum distribution of category 3 pion tracks. Figures (b) and (c) show the momenta of category $7 \& 15$ proton tracks respectively.

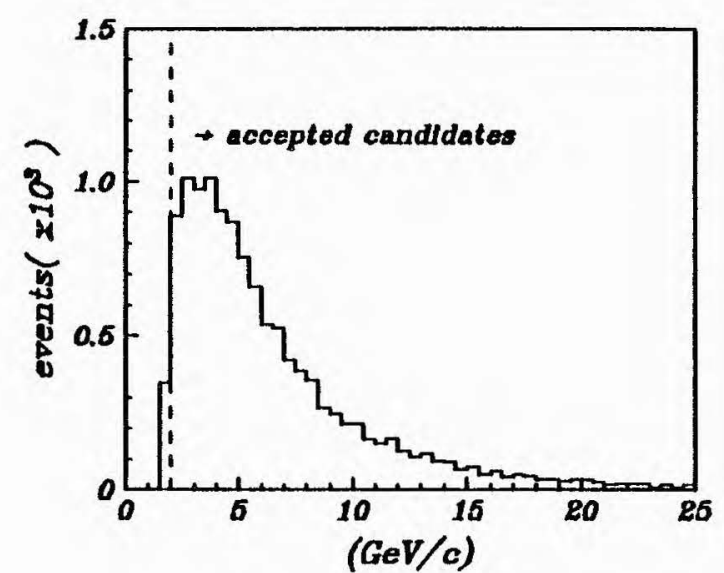

Figure 3-7(b)

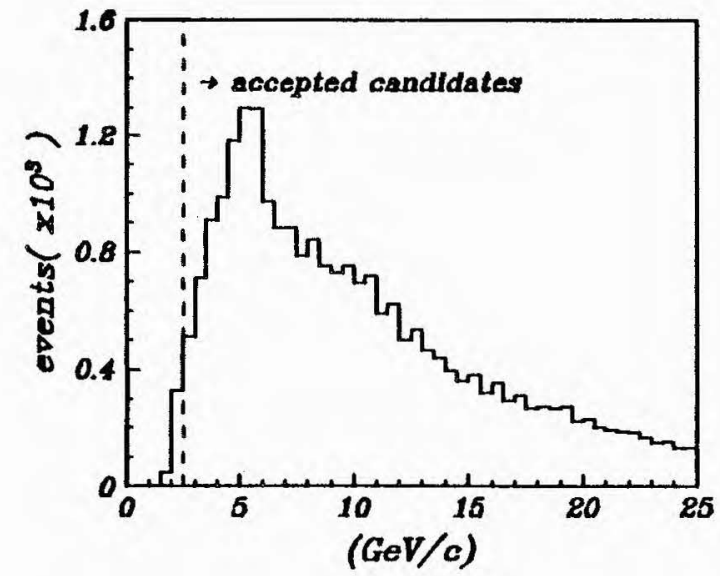

Figure 3-7(c) 


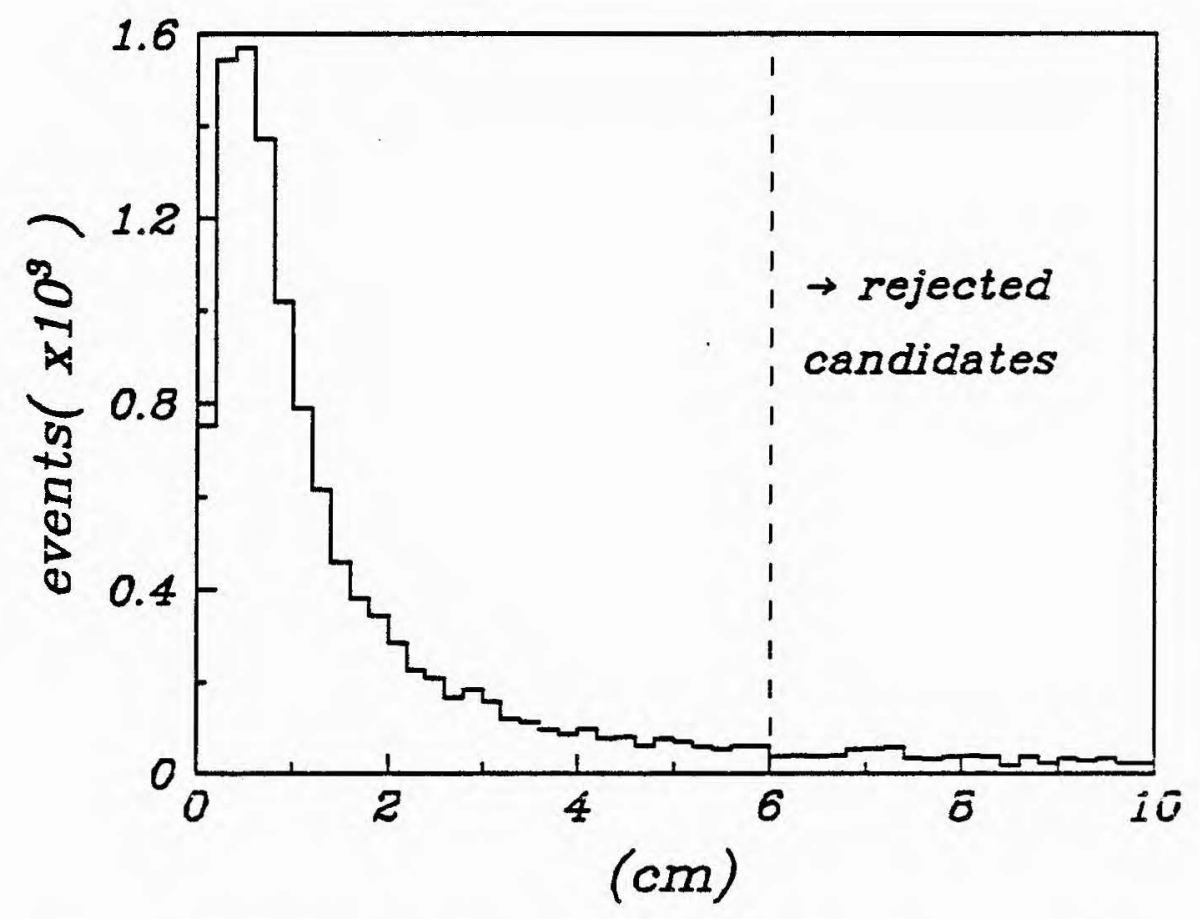

Figure 3-8 Distribution of distance-of-closest-approach between a $\Lambda$ candidate and the main production vertex for poor proton tracks. 


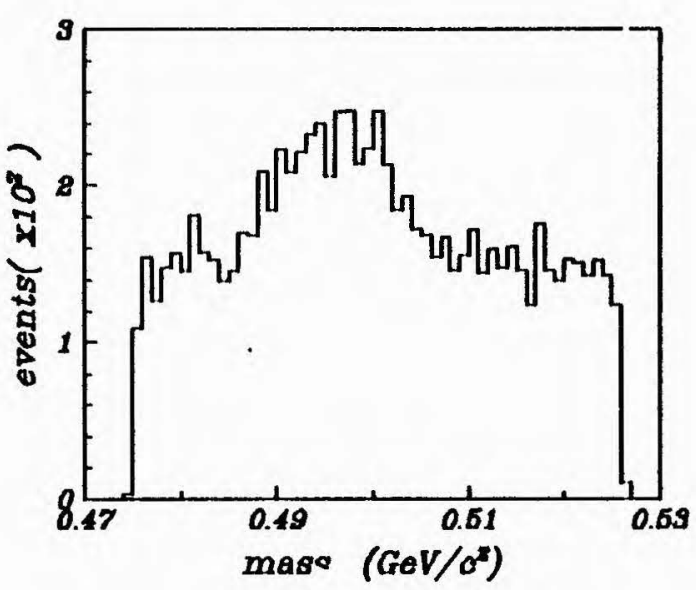

Figure 3-9(a) Displays of those $\Lambda$ candidates that are also $K_{s}^{0}$ candidates. Figure (a) is the mass plot when a pion mass hypothesis is used for the proton track. Note the $K^{0}$ peak centered at $498 \mathrm{MeV} / \mathrm{c}^{2}$. Figure (b) shows those $\Lambda$ candidates that are rejected by the cut while (c) displays those candidates iccepted by the same cut.

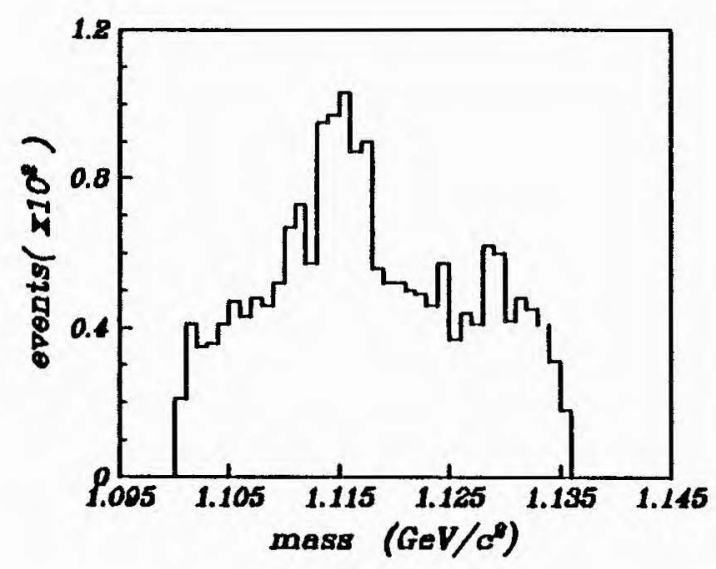

Figure 3-9(b)

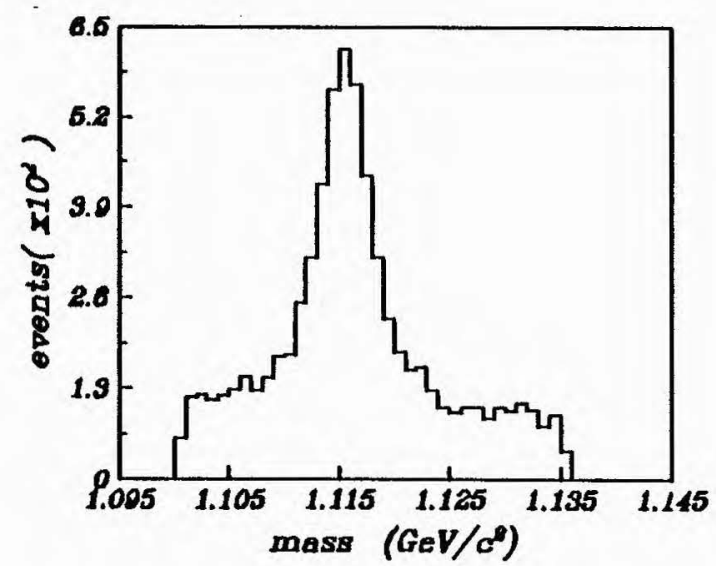

Figure 3-9(c) 


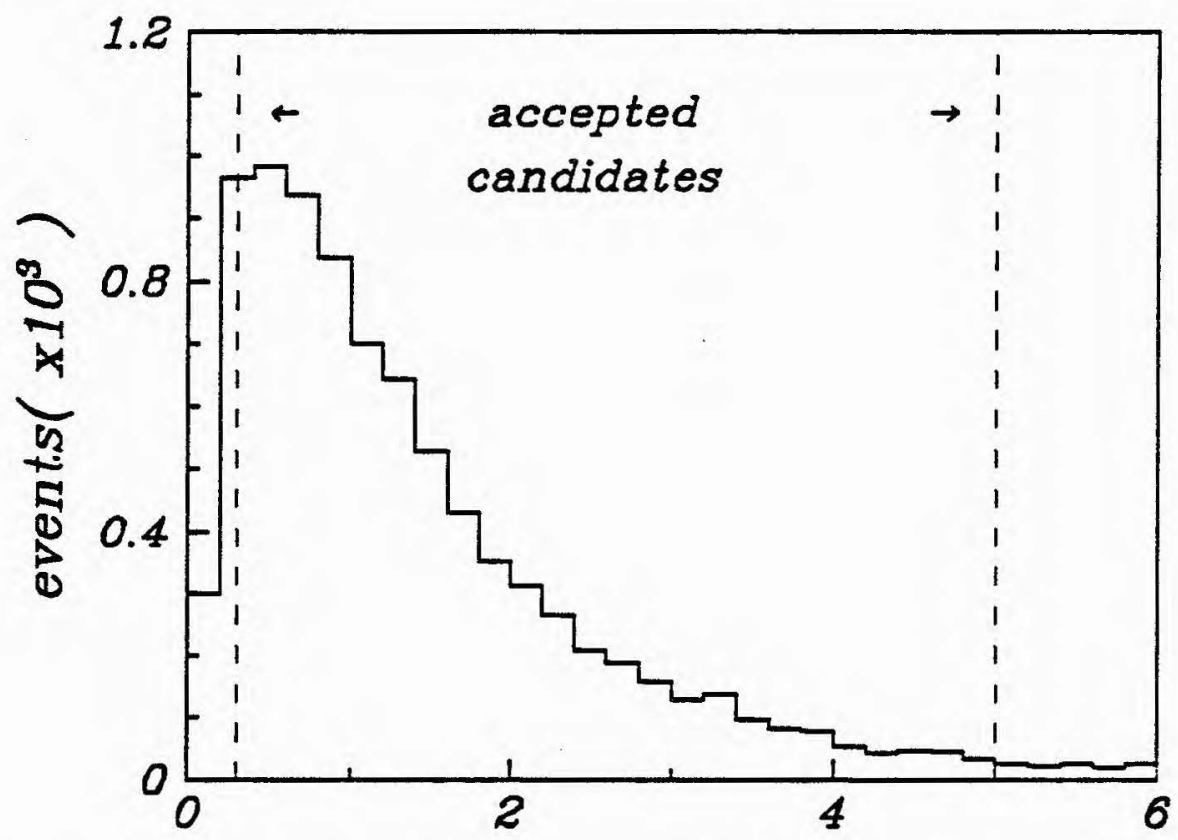

Figure 3-10 The distribution of the ratio of the measured distance from the main vertex and the expected mean decay distance (in the laboratory frame of reference) for $\Delta$ candidates with both tracks being of the category 3 variety. 


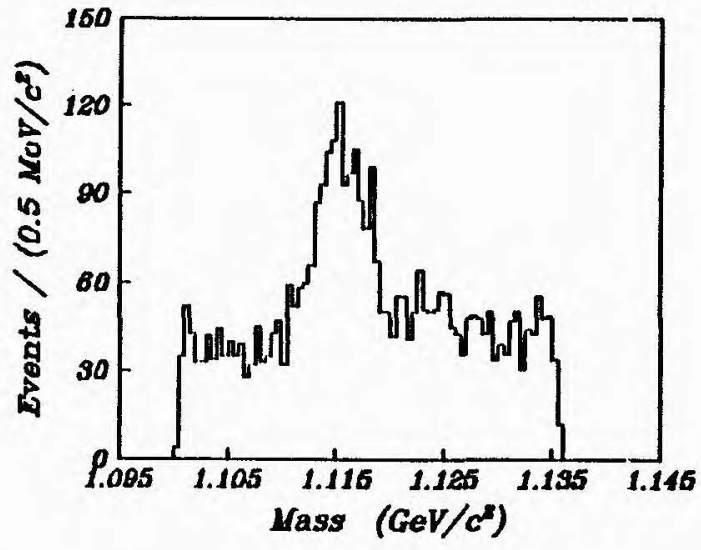

Figure 3-11(a) (a) The subset of $\Lambda$ candidates with track duplication. Figure (b) shows those events accepted by the particle identification cut while (c) displays the rejected events.

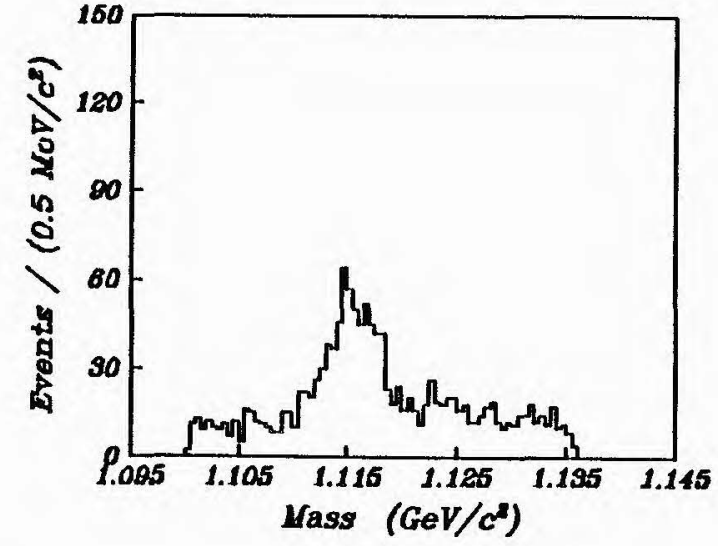

Figure 3-11(b)

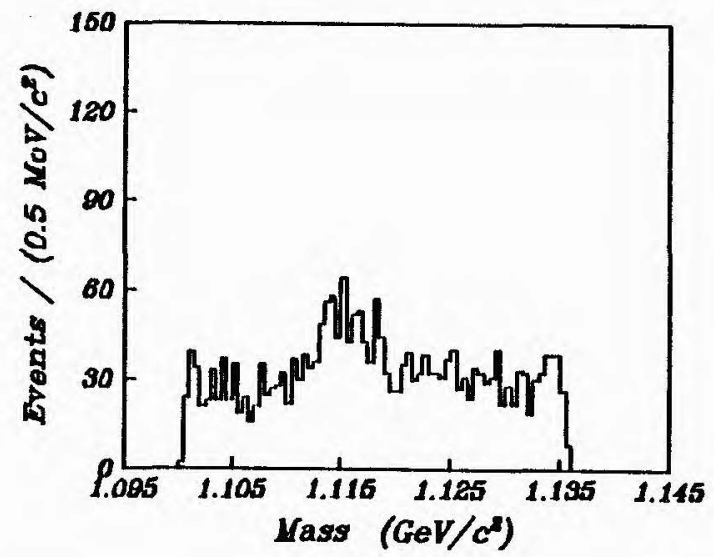

Figure 3-11(c) 


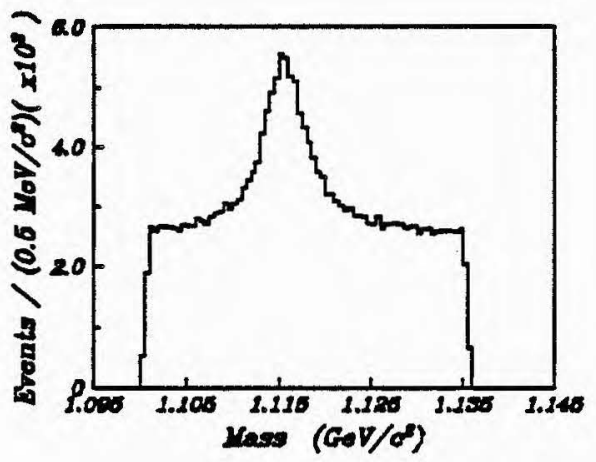

Figure 3-12(a) Signal after cut on DCA of $(p, \pi)$ pair.

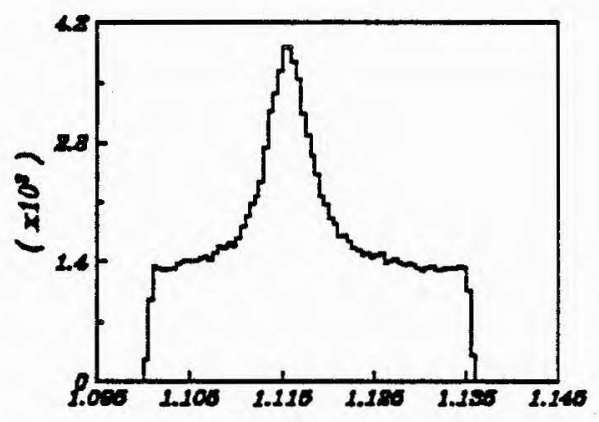

Figure 3-12(c) The signal after the proton identification cut.

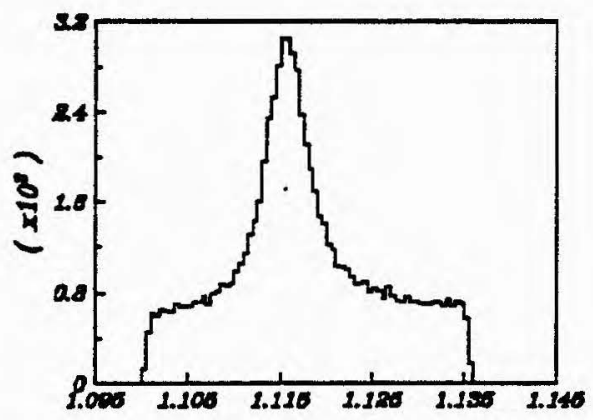

Figure 3-12(e) The final $\Lambda / \bar{\Lambda}$ signal.

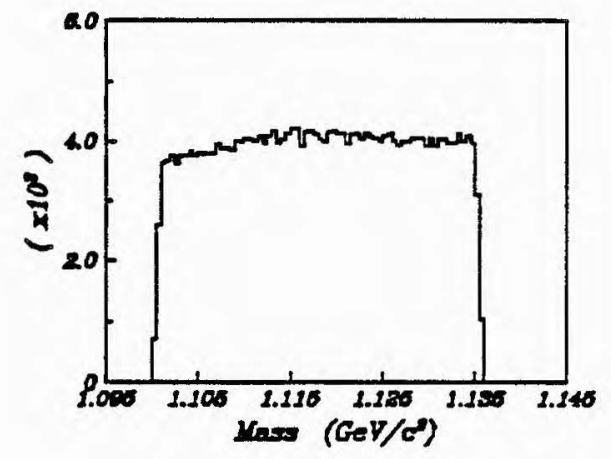

Figure 3-12(b) Background to cut used in (a).

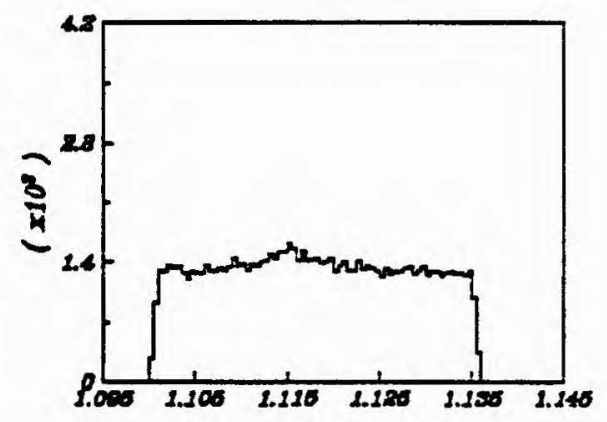

Figure 3-12(d) Background to cut of (c).

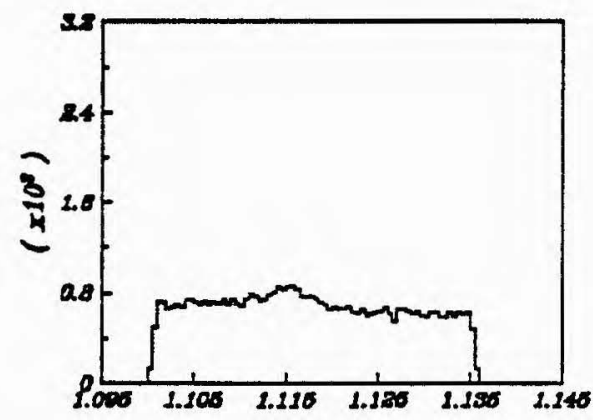

Figure 3-12(f) Background to all cuts used after (c). 


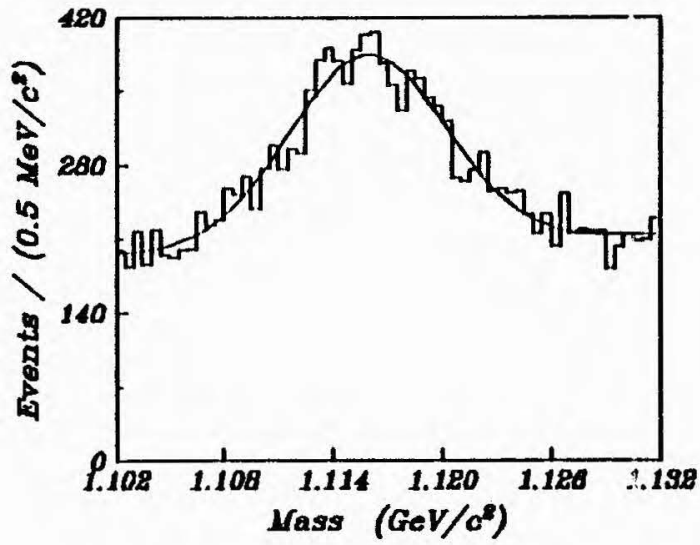

Figure 3-13(a) Plots of $\Lambda$ signal fit to Ganssian signal function plus a linear background. In (a) both tracks are poor quality. Only one track is poor in (b), while both tracks are good ir (c).

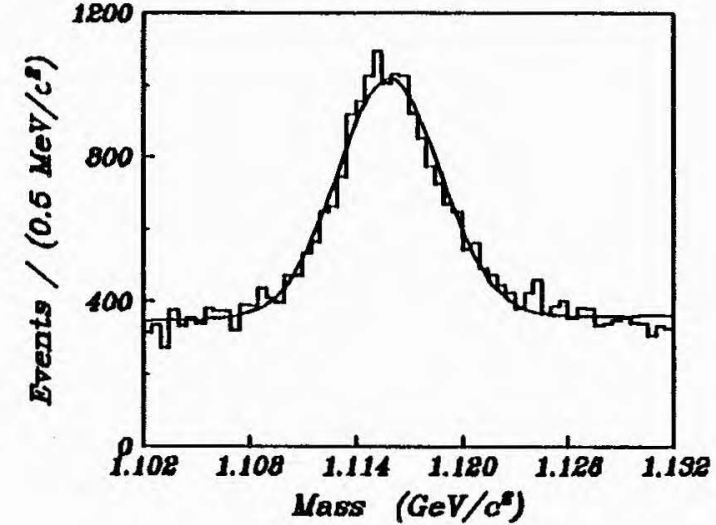

Figure 3-13(b)

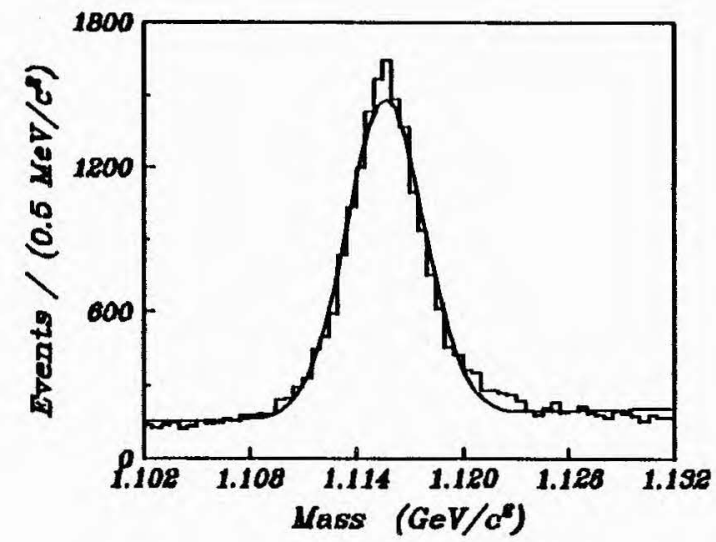

Figure 3-13(c) 


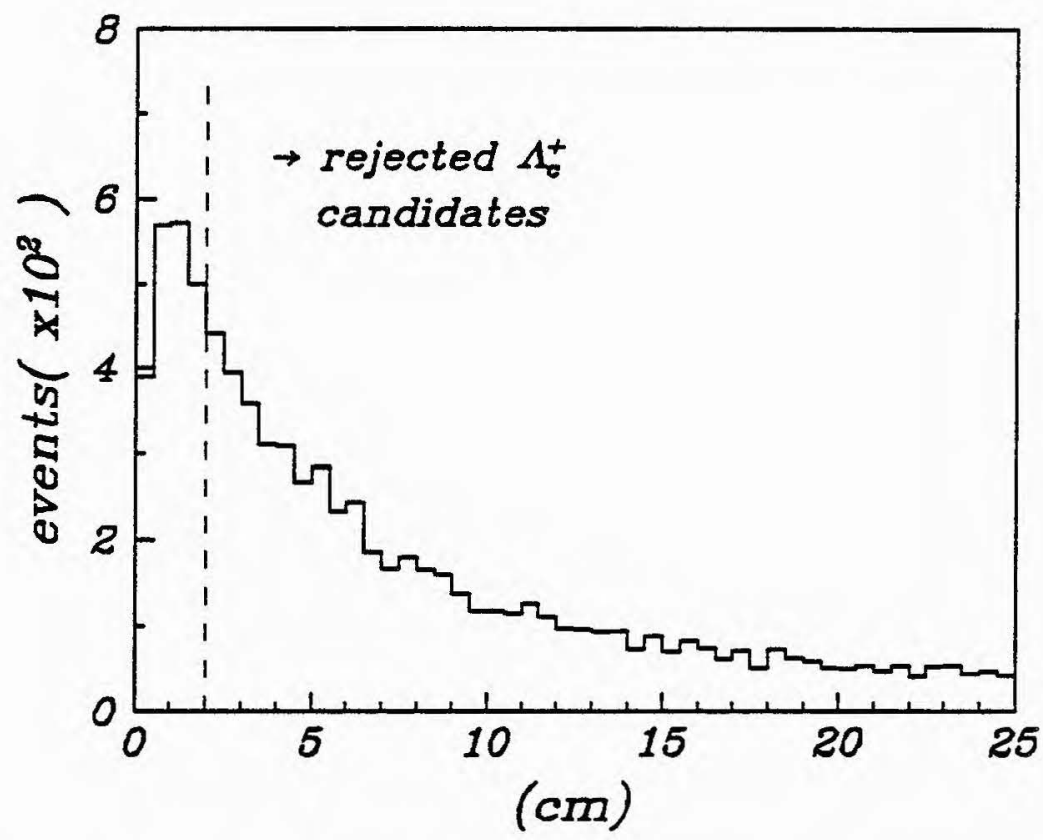

Figure 3-14 The distance between the main vertex and the secondary vertex formed by a $\left(\Lambda, \pi^{+}\right)$combination. Since the $\Lambda_{c}^{+}$has a small lifetime, it will decay a very short distance from the production vertex.

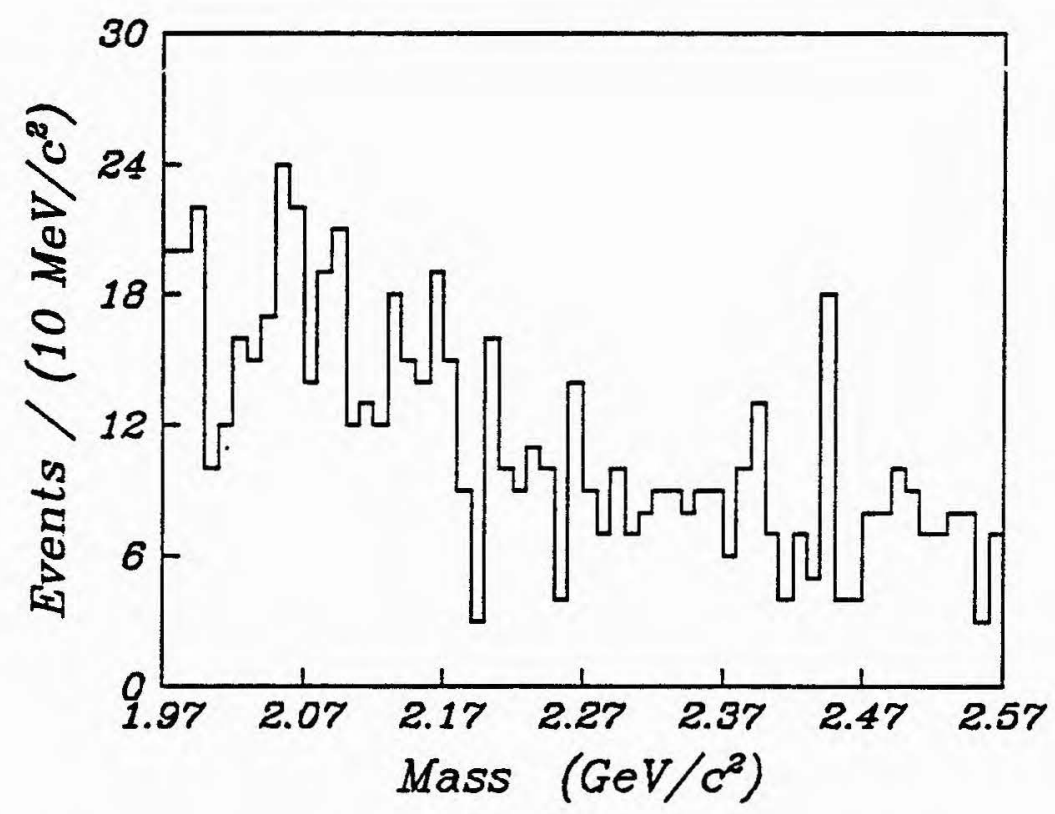

Figure 3-15 Those $\left(\Lambda, \pi^{+}\right)$events rejected as containing a well-identified recoil proton in the recoil detector. 


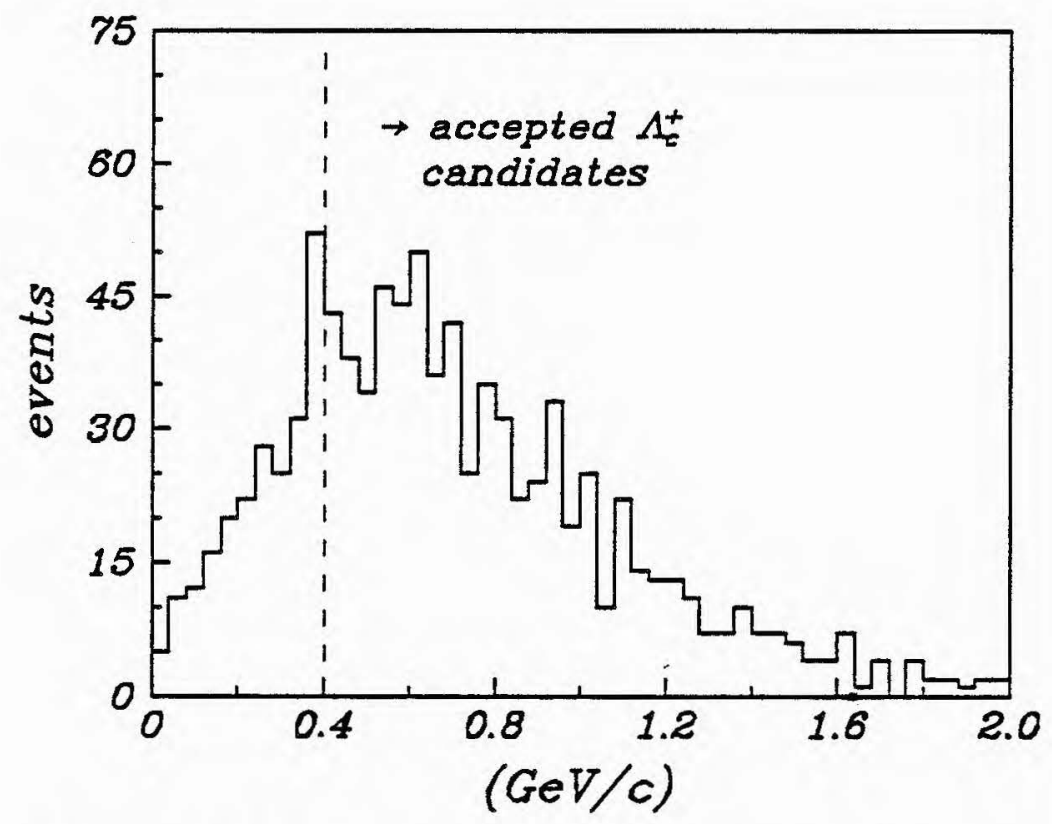

Figure 3-16 The transverse momentum distribution for the $\left(\Lambda, \pi^{+}\right)$events of figure 3-18(d).

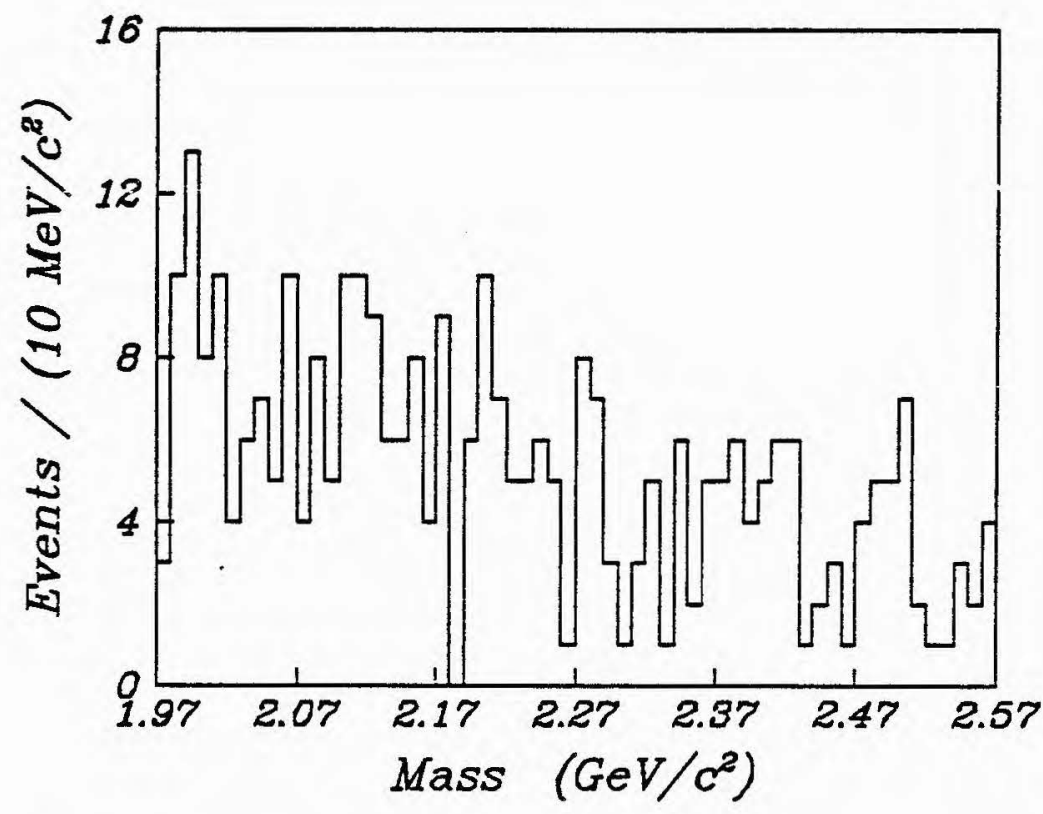

Figure 3-17 Those $\left(\Lambda, \pi^{+}\right)$events with a transverse momentum $P_{T}<0.4 \mathrm{GeV} / \mathrm{c}$. 


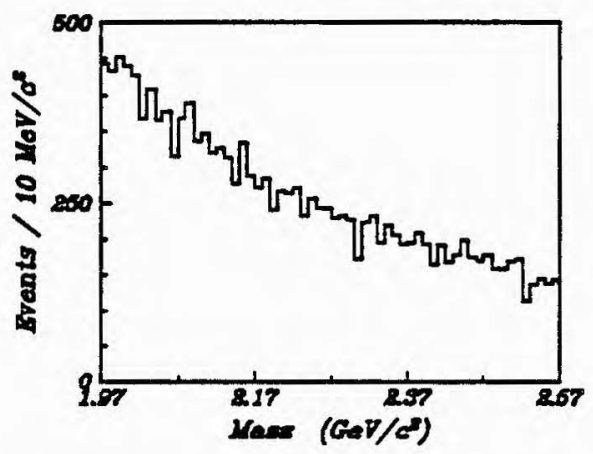

Figure 3-18(a) All $\left(\Lambda, \pi^{+}\right)$events.

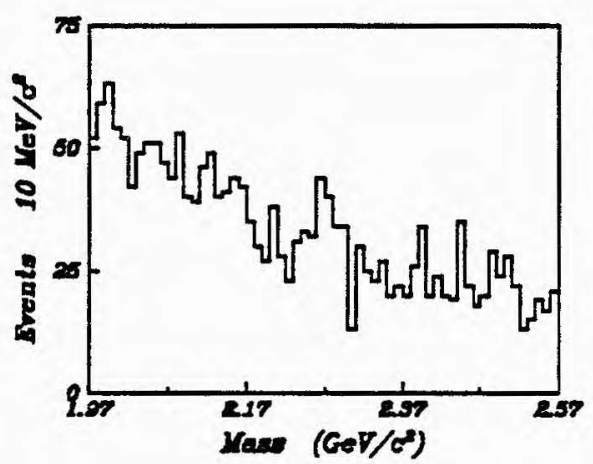

Figure 3-18(c) After $\Lambda$ mass cut of section $(3-3-2)$.

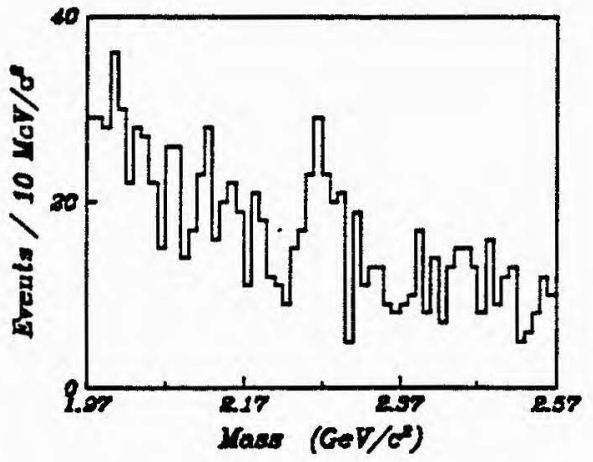

Figure 3-18(e) After $P_{T}$ cut of section (3$3-4)$.

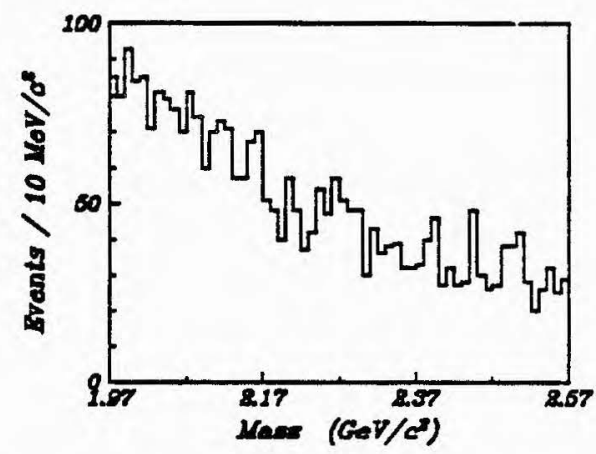

Figure 3-18(b) After vertex cut of section (3-3-1).

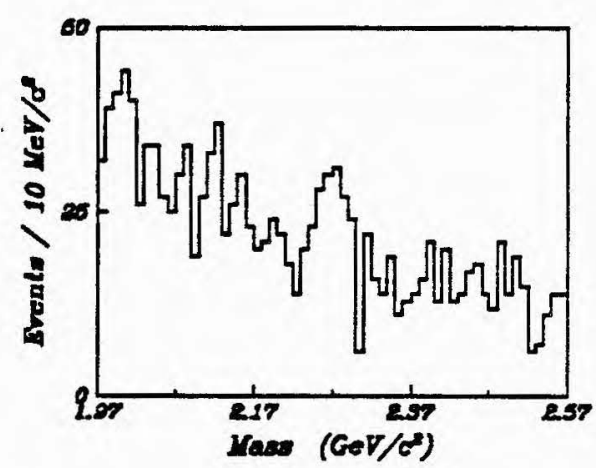

Figure 3-18(d) After recoil proton requirement of section (3-2 3$)$.

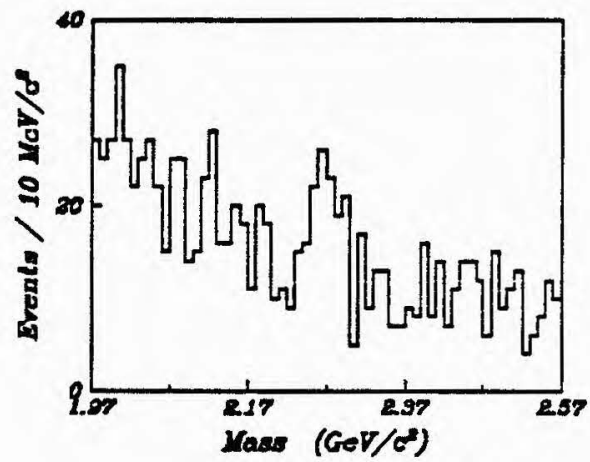

Figure 3-18(f) Final mass plot after $\Lambda$ and photon energy cuts of section (3-3-5). This plot is also reproduced in figure $3-19(\mathrm{a})$. 


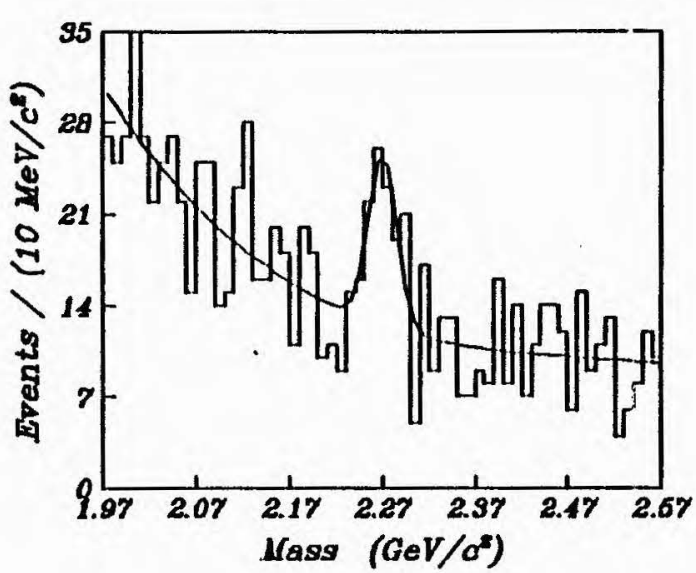

Figure 3-19(a) $\left(\Lambda, \pi^{+}\right)$fit to Gaussian signal plus cubic polynomial background.

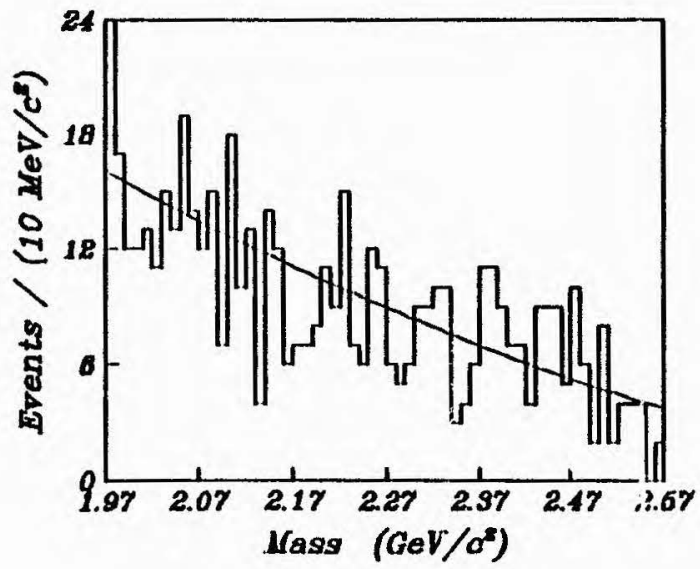

Figure 3-19(c) $\left(\bar{\Lambda}, \pi^{+}\right)$

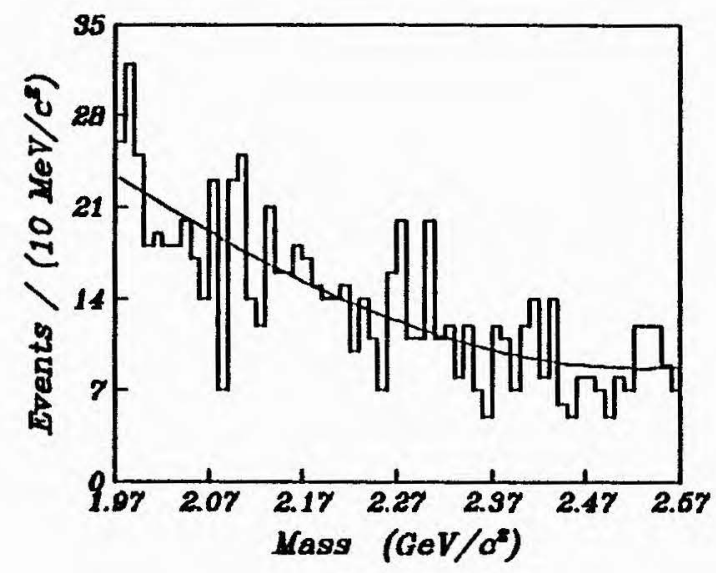

Figure 3-19(b) $\left(\Lambda, \pi^{-}\right)$fit to cubic polynomial background. The same applies to (c) \& (d).

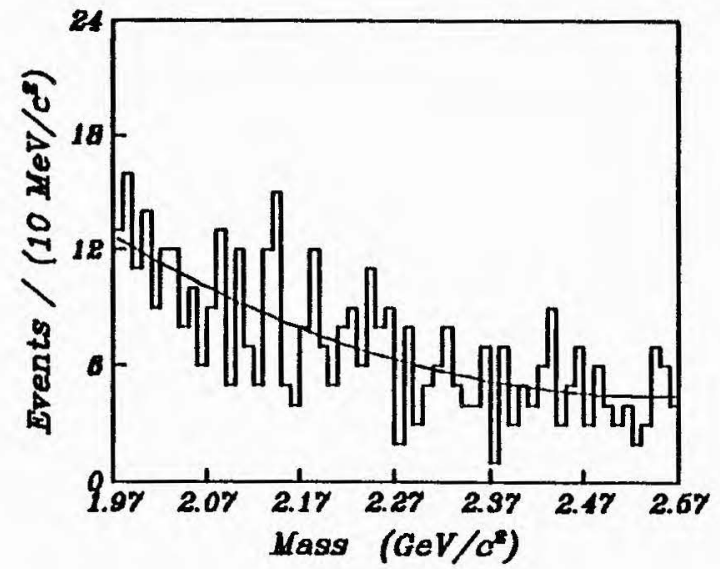

Figure 3-19(d) $\left(\bar{\Lambda}, \pi^{-}\right)$ 


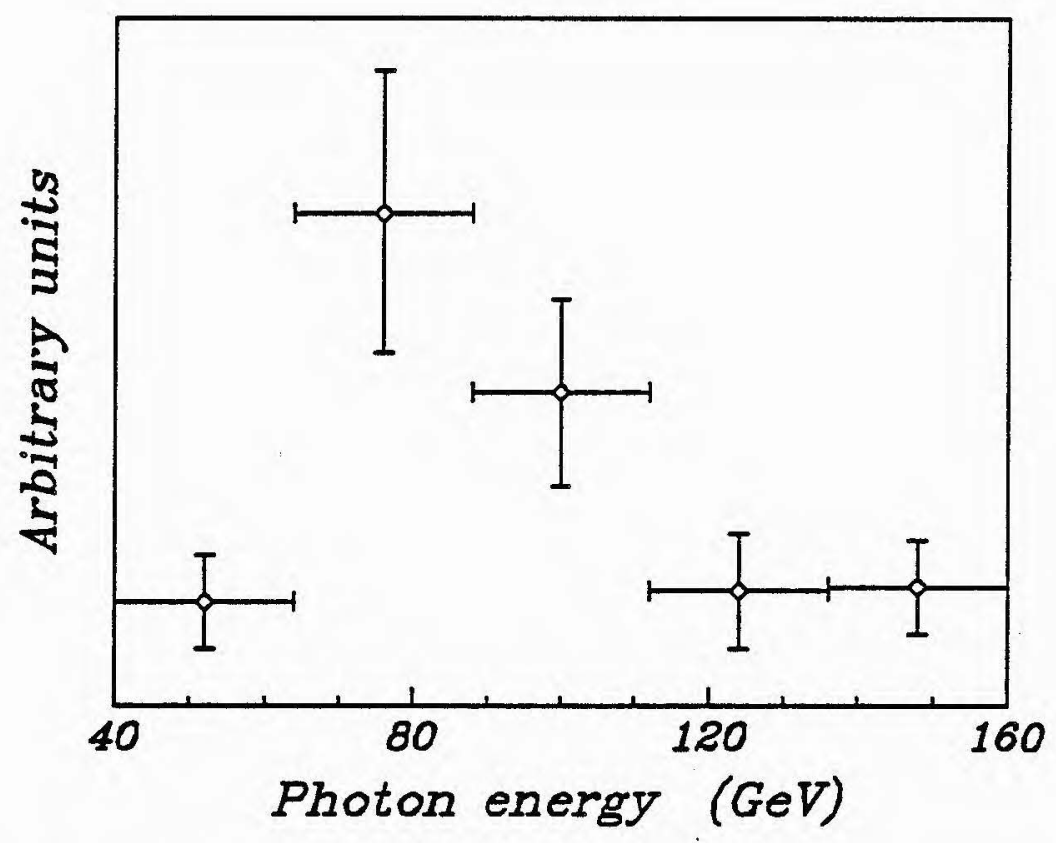

Figure 3-20(a) Variation of the $\Lambda_{c}^{+}$signal as a function of the incident photon beam energy. The data has been corrected by the acceptance function displayed in figure 3-20(b) below. This includes normalization to the incident beam flux.

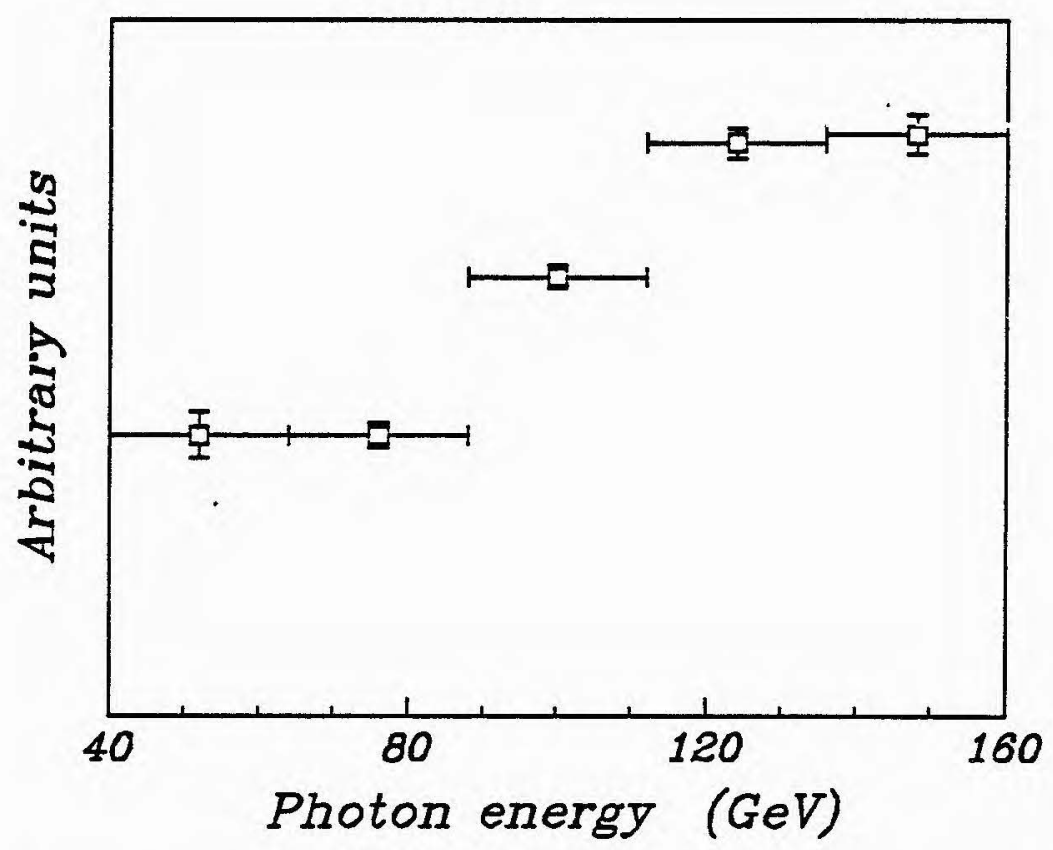

Figure 3-20(b) Acceptance for the $\Lambda_{c}^{+}$signal as a function of beam energy. The errors in the ordinate are statistical only. The acceptance includes normalization to the incident beam flux as shown in figure 2-6. 


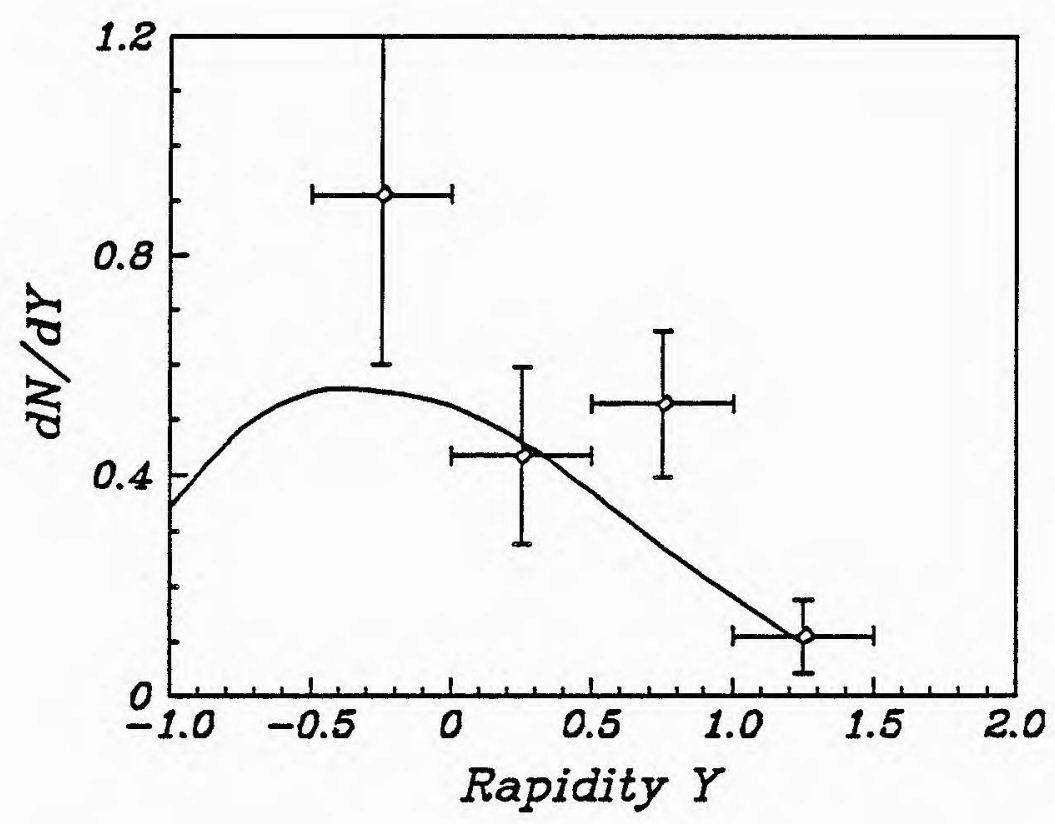

Figure 3-21(a) Rapidity distribution of the $\Lambda_{c}^{+}$in the $\gamma p$ center-of-mass frame acceptance corrected by figure 3$21(\mathrm{~b})$. The solid curve is the theoretical $\Lambda_{c}^{+}$rapidity distribution displayed in figure 1-5.

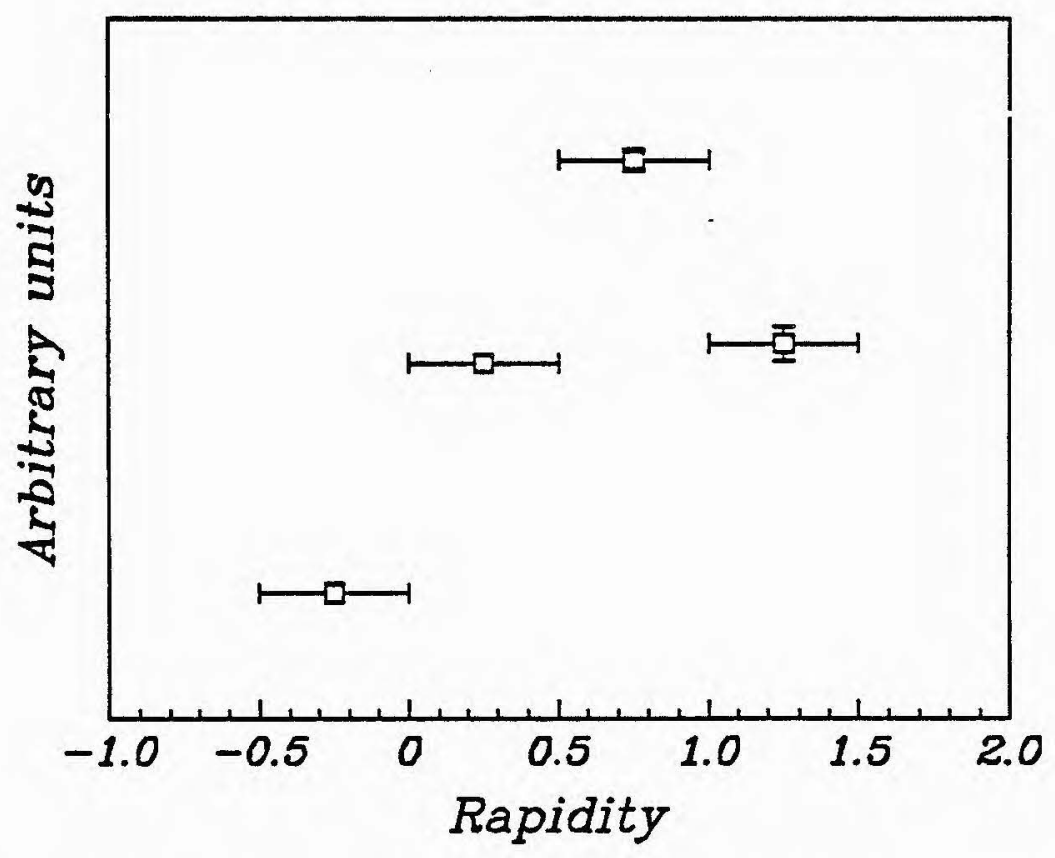

Figure 3-21(b) Acceptance for the $\Lambda_{c}^{+}$rapidity. 


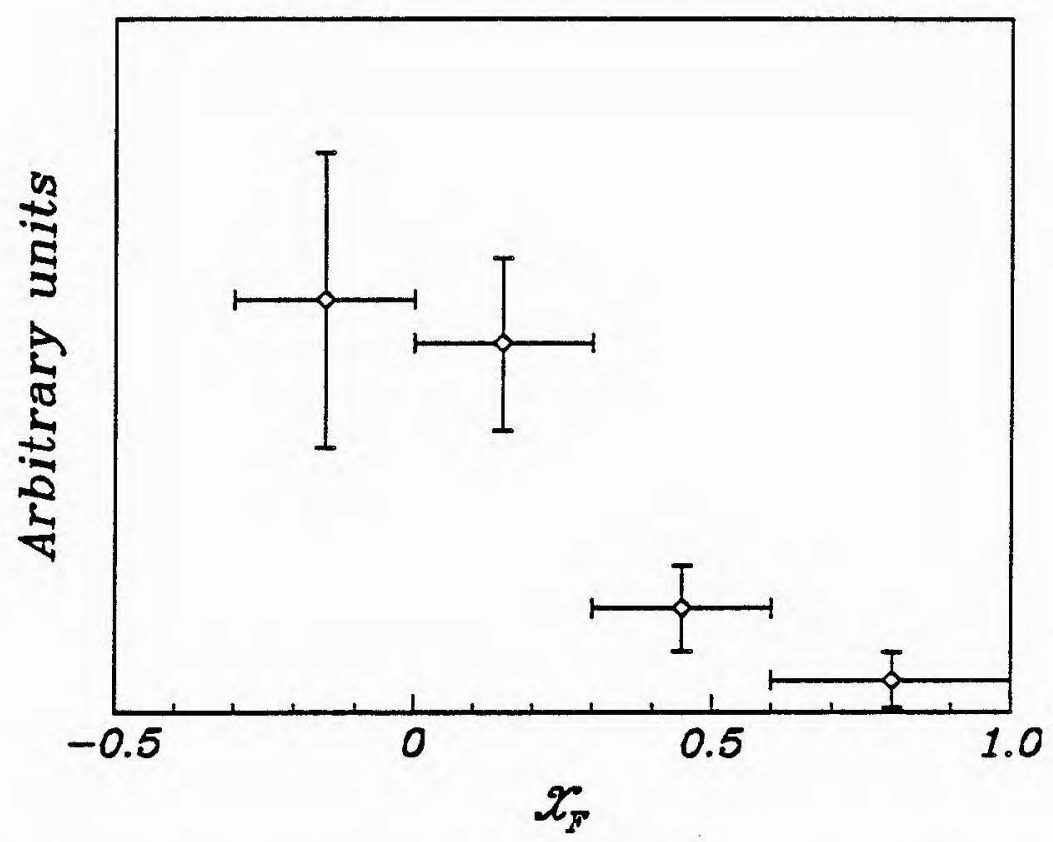

Figure 3-22(a) Feynman $x_{F}$ distribution for the $\Lambda_{c}^{+}$ acceptance corrected by figure 3-22(b).

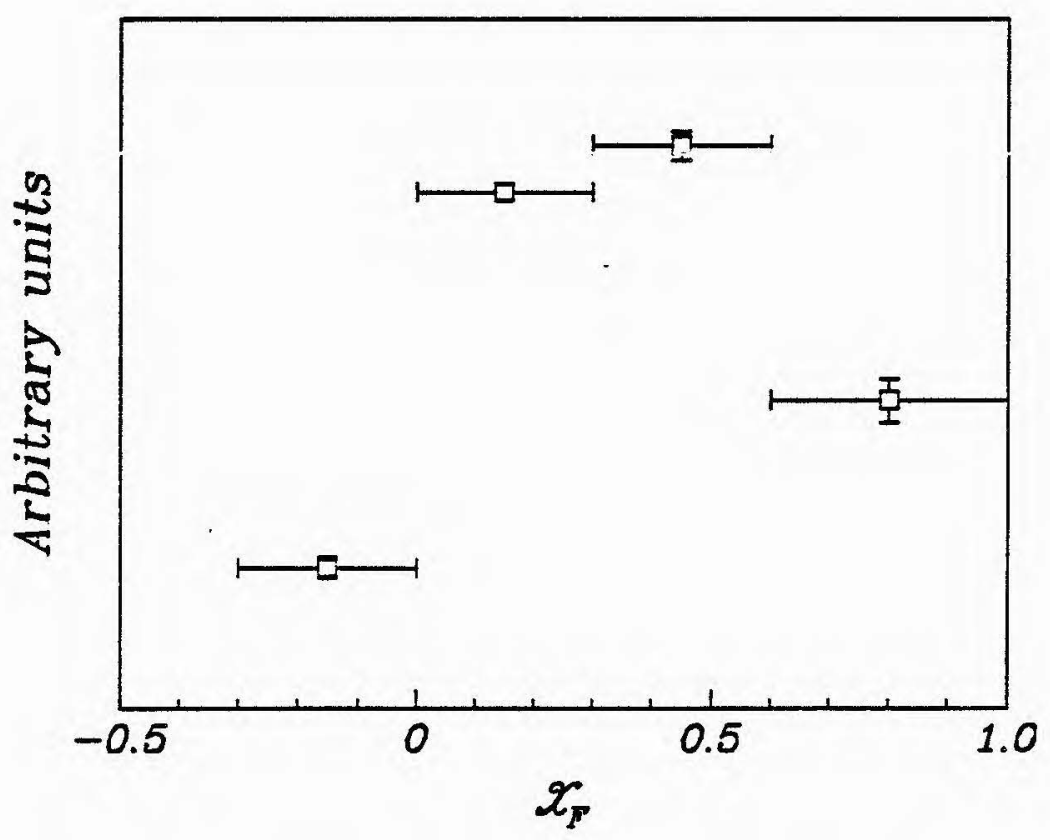

Figure 3-22(b) Acceptance in $x_{F}$ for the $\Lambda_{c}^{+}$. 


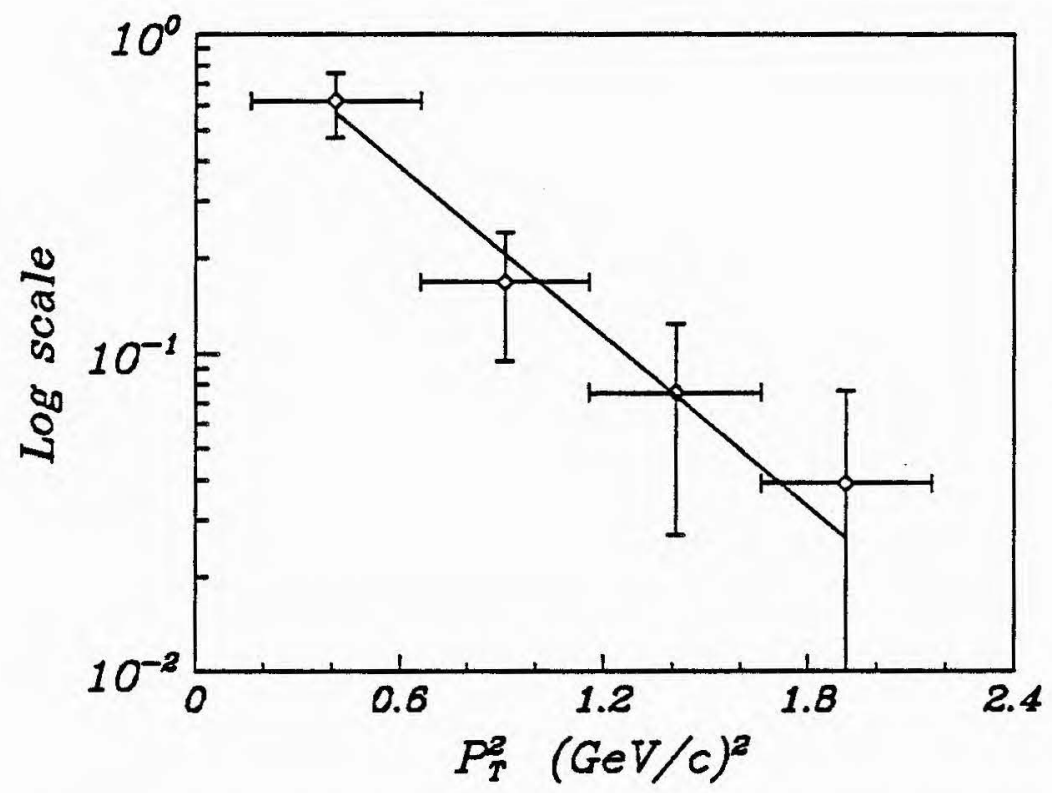

Figure 3-23(a) $P_{\tau}^{2}$ of the $\Lambda_{c}^{+}$signal. The slope was found to be $-2.0 \pm 0.9(\mathrm{GeV} / \mathrm{c})^{-2}$.

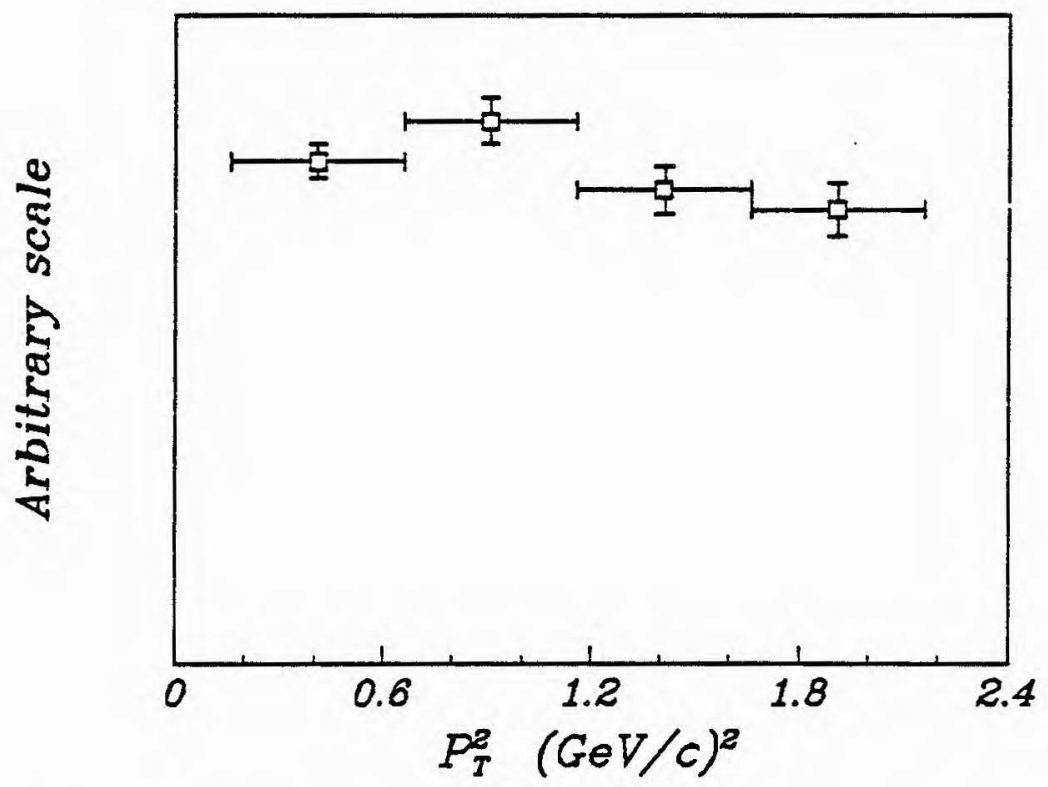

Figure 3-23(b) Acceptance in $P_{T}^{2}$ for the $\Lambda_{c}^{+}$. 


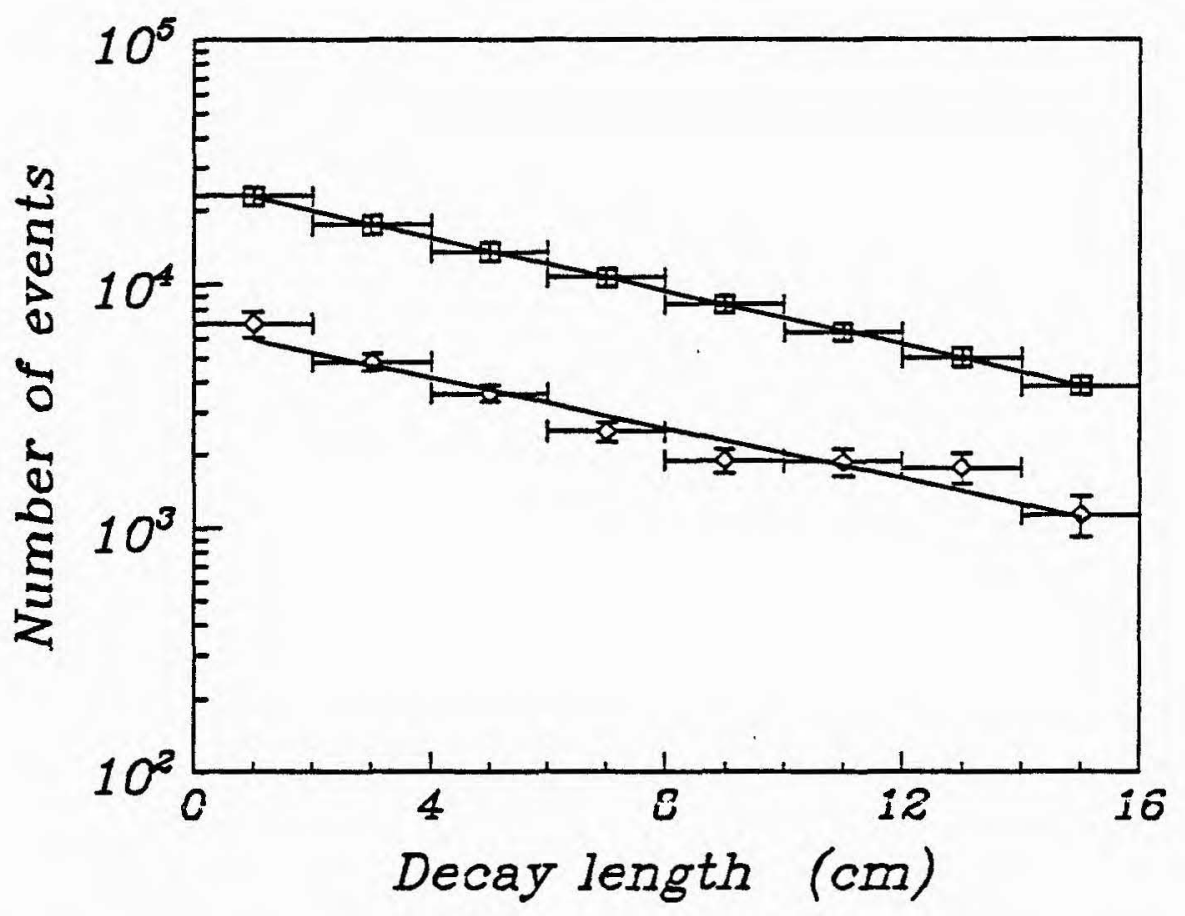

Figure 3-24 Reconstruction of the $\Delta$ lifetime using the Toronto version of the E516 Monte Carlo. The upper set of data represents the proper decay length of the hyperon as generated by the Monte Carlo. A fit to this simulated data produces a value $c \tau=7.8 \pm 0.6 \mathrm{~cm}$. Below this is the reconstructed decay length for the $\Lambda$ hyperons taken from the data. The data distribution has been corrected for the acceptance losses simulated by the Monte Carlo. The resultant slope is $c \tau=8.4 \pm 1.0 \mathrm{~cm}$. The accepted value for the $\Delta$ is $7.89 \pm 0.06 \mathrm{~cm} \mathrm{[1]}$. 


\section{References}

[1] Particle Data Group, M. Aguilar-Benitez et al., "Review of particle properties", Phys. Lett. 170B (1986).

[2] E. G. Cazzoli et al., "Evidence for $\Delta S=-\Delta Q$ Currents or Charmed Baryon Production by Neutrinos", Phys. Rev. Lett. 341125 (1975).

[3] B. Knapp et al., "Observation of a Narrow Antibaryon State at $2.26 \mathrm{GeV} / c^{2}$ ", Phys. Rev. Lett. 37882 (1976).

[4] T. K. Gaisser and F. Halzen, "Long-lived tracks in emulsions: New hadrons or background?", Phys. Rev. D 143153 (1976).

[5] S. J. Barish et al., "Properties of a neutrino-induced dilepton event", Phys. Rev. D 151 (1977).

[6] B. W. Lee, C. Quigg, and J. L. Rosner, "Charmed baryon interpretation of $\bar{\Lambda} \pi^{-} \pi^{-} \pi^{+}$and $\bar{\Lambda} \pi^{-} \pi^{-} \pi^{+} \pi^{ \pm}$peaks", Phys. Rev. $D 15157$ (1977).

[7] C. Angelini et al., "On the Lifetime of Charged Charmed Particles and First Direct Observation of a Charmed Baryon Decay.", Phys. Lett. 84B 150 (1979).

[8] A. M. Cnops et al., "Observation of the Kaonic Decay of the $\Lambda_{c}^{+}$Charmed Baryon", Phys. Rev. Lett. 42197 (1979).

[9] C. Baltay et al., "Confirmation of the Existence of the $\Sigma_{c}^{++}$and $\Lambda_{c}^{+}$Charmed Baryons, and Observation of the Decay $\Lambda_{c}^{+} \rightarrow \Lambda \pi^{+}$and $\bar{K}^{0} p$ ", Phys. Rev. Lett. 421721 (1979).

[10] ACCDHW Collab., D. Drijard et al., "Charmed Baryon Production at the CERN Intersecting Storage Rings", Phys. Lett. 85B 452 (1979).

[11] K. L. Giboni et al., "Diffractive Production of the Charmed Baryon $\Lambda_{c}^{+}$at the CERN ISR", Phys. Lett. 85B 437 (1979).

[12] W. Lockman et al., "Evidence for $\Lambda_{c}^{+}$in Inclusive $p p \rightarrow\left(\Lambda^{0} \pi^{+} \pi^{+} \pi^{-}\right)+X^{\text {", }}$, Phys. Lett. 85B 443 (1979).

[13] G. S. Abrams et al., "Observation of Charmed Baryon Production in $e^{+} e^{-}$ Annihilation", Phys. Rev. Lett. 4410 (1980).

[14] D. Allasia et al., "Investigation of the Decay of Charmed Particles produced in Neutrino Interactions", Nucl. Phys. B176 13 (1980).

[15] M. Calicchio et al., "First Observation of the Production and Decay of the $\Sigma_{c}^{+} "$, Phy. Lett. 93B 521 (1980).

[16] T. Kitagaki et al., "Charmed baryon Production in High-Energy NeutrinoDeuterium Interactions", Phys. Rev. Lett. 45955 (1980). 
[17] N. Ushida et al., "Measurement of $D^{+}, F^{+}$, and $\Lambda_{c}^{+}$Charmed Particle Lifetimes", Phys. Rev. Lett. 451053 (1980).

[18] H. C. Ballagh et al., "Dilepton Production by neutrinos in the Fermilab 15-foot bubble chamber", Phys. Rev. D 247 (1981).

[19] M. Basile et al., "A Measurement of Two Resonant Contributions in the $\Lambda_{c}^{+}$ Branching Ratios", Nuov. Cim. 62A 14 (1981).

[20] Photon-Emulsion Collab., A. Forino et al., "Associated Photoproduction of a Charmed Meson and a Charmed Baryon", Lett. Nuov. Cim. 30166 (1981).

[21] Photon-Emulsion Collab., M. I. Adamovitch et al., "Observation of Pairs of Charmed Particles produced by High-Energy Photons in Nuclear Emulsions coupled with a Magnetic Spectrometer", Phys. Lett. 99B 271 (1981).

[22] H. Grässler et al., "Observation of Charmed Baryon Production in $\nu p$ Interactions in BEBC", Phys. Lett. 99B 159 (1981).

[23] J. Irion et al., "Production of Charmed Particles at the CERN Intersecting Storage Rings in Events triggered by an Electron", Phys. Lett. 99B 495 (1981).

[24] J. J. Russell et al., "Photoproduction of Charmed Baryons", Phys. Rev. Lett. 46799 (1981).

[25] BIS-2 Collab., A. N. Aleev et al., "Observation of charmed $\Lambda_{c}^{+}$baryons produced in the neutron beam of the Serpukov accelerator", Sov. J. Nucl. Phys. 35687 (1982).

[26] P. C. Bosetti et al., "Observation of a Doubly Charged Charmed Baryon in the

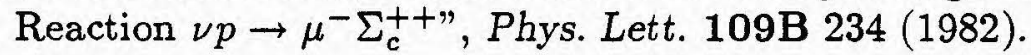

[27] T. Kitagaki et al., "New Decay Mode of the Charmed Baryon $\Lambda_{c}^{+} \rightarrow \Sigma^{0} \pi^{+}$", Phys. Rev. Lett. 48299 (1982).

[28] D. Son et al., "Charmed Baryon Producion in $\nu d \rightarrow \mu^{-} \Lambda X$ Reactions", Phys. Rev. Lett. 491128 (1982).

[29] E. Vella et al., "Observation of Semileptonic Decays of Charmed Baryons", Phys. Rev. Lett. 481515 (1982).

[30] D. DiBitonto, "Data on Charm and Beauty Production at the CERN ISR", AIP Conference Proceedings on Proton-Antiproton Collider Physics, edited by V. Barger, D. Cline, and F. Halzen, Madison, Wisconsin, p. 26 (1981).

[31] N. Ushida et al., "New Results for the Lifetimes of the $D^{ \pm}, F^{ \pm}$, and $\Lambda_{c}^{+}$Particles", Phys. Rev. Lett. 512362 (1983).

[32] S. F. Biagi et al., "Observation of a Narrow State at $2.46 \mathrm{GeV} / c^{2}-\mathrm{A}$ Candidate for the Charmed Strange Baryon $A^{+"}$, Phys. Lett. 122B 455 (1983). 
[33] K. Abe et al., "Charm photoproduction at $20 \mathrm{GeV",} \mathrm{Phys.} \mathrm{Rev.} \mathrm{D} 301$ (1984).

[34] Photon-Emulsion Collab., M. I. Adamovich et al., "Charged Charmed Particle Lifetimes", Phys. Lett. 140B 119 (1984).

[35] BIS-2 Collab., A. N. Aleev et al., "The $\Lambda_{c}^{+}$Production by $40-70 \mathrm{GeV}$ Neutrons on Carbon", Z. Phys. C 23333 (1984).

[36] S. F. Biagi et al., "Properties of the Charmed Strange Baryon $A^{+}$and Evidence for the Charmed Doubly Strange Baryon $T^{0}$ at $2.74 \mathrm{GeV} / c^{2}$ ", Z. Phys. $C 28$ 175 (1985).

[37] T. Bowcock et al., " $\Lambda_{c}^{+}$Production from $e^{+} e^{-}$Annihilation in the $\Upsilon$ Energy Region", Phys. Rev. Lett. 55923 (1985).

[38] K. Abe et al., "Lifetimes, cross sections, and production mechanisms of charmed particles produced by $20 \mathrm{GeV}$ photons", Phys. Rev. D 331 (1986).

[39] N. Ushida et al., "The Lifetimes of the Charmed-Particles $D^{ \pm}, F^{ \pm}$, and $\Lambda_{c}^{+}$ produced by Neutrinos", to be published in Physical Review Letters (1986).

[40] H. Seywerd, private communication.

[41] The Photon Emulsion Collab., M. I. Adamovich et al., "Cross Sections and some features of Charm Photoproduction at $\gamma$ energies of $20-70 \mathrm{GeV}$ ", submitted to Physics Letters B, CERN/EP 86-69.

[42] D. Aston et al., "Photoproduction of Charmed $D$ Mesons at $\gamma$ energies of 40-70 GeV", Phys. Lett. 94B 113 (1980).

[43] K. Abe et al., "Charm Photoproduction Cross Section at $20 \mathrm{GeV",} \mathrm{Phys.} \mathrm{Rev.}$ Lett. 51156 (1983).

[44] A. Kernan and G. Van Dalen, "Charm and Beauty Production in Strong Interactions", Phys. Rep. 106297 (1984).

[45] W. C. Louis, "Where We Stand with Charmed Baryons", AIP Conference Proceedings on Hadron Spectroscopy, edited by S. Oneda, University of Maryland, p. 313 (1985).

[46] A. L. Duncan, "Characteristics of Hadronic States observed in High Energy Diffractive Photoproduction in Hydrogen", Ph. D. thesis, University of Colorado, Boulder (1982).

[47] B. D. Denby, "Inelastic and Elastic Photoproduction of $J / \psi(3097)$ ", Ph. D. thesis, University of California, Santa Barbara (1983).

[48] D. J. Summers, "A Study of the Decay $D^{0} \rightarrow K^{-} \pi^{+} \pi^{0}$ in High Energy Photoproduction", Ph. D. thesis, University of California, Santa Barbara (1984). 
[49] S. Bhadra, "Inclusive Distributions of Diffractively Produced Neutral Kaons, Lambdas, and Antilambdas, and Upper Limits on $\Lambda_{c}^{+}$Production in HighEnergy $\gamma p$ Interactions", $P h . D$. thesis, University of Colorado, Boulder (1984).

[50] J. P. Cumalat, "Measurements of the Photon Total Cross Section on Protons from $45 \mathrm{GeV}$ to $182 \mathrm{GeV}$ ", Ph. D. thesis, University of California, Santa Barbara (1977).

[51] R. M. Egloff, "Measurements of Elastic Rho, Omega, and Phi Meson Photoproduction Cross Sections on Protons from 30 to $180 \mathrm{GeV}$ ", Ph. D. thesis, University of Toronto, Toronto (1979).

[52] J. J. Russell, "Photoproduction of Charmed Baryons", Ph. D. thesis, University of Illinois, Urbana-Champaign (1980).

[53] E. N. Vella, "Charmed Baryon Decays observed in $e^{+} e^{-}$Annihilation", Ph. $D$. thesis, University of California, Berkeley (1981).

[54] G. F. Hartner et al., "A Recoil Proton Detector using Cylindrical Proportional Chambers and Scintillation Counters", Nucl. Instr. Meth. 216113 (1983).

[55] D. J. Summers et al., "Study of the Decay $D^{0} \rightarrow K^{-} \pi^{+} \pi^{0}$ in High-Energy Photoproduction", Phys. Rev. Lett. 52410 (1984).

[56] B. H. Denby et al., "Inelastic and Elastic Photoproduction of $J / \psi(3097)$ ", Phys. Rev. Lett. 52795 (1984).

[57] K. Sliwa et al., "Study of $D^{*}$ production in high-energy $\gamma p$ interactions", Phys. Rev. D 321053 (1985).

[58] D. J. Summers, "Reconstruction of a Strip Geometry Calorimeter using Stepwise Regression", Nucl. Instr. Meth. 228290 (1985).

[59] S. Bhadra et al., "Study of Inclusive $K_{s}^{0}, \Lambda$, and $\bar{\Lambda}$ Production in Diffractive $\gamma p$ Interactions", Phys. Rev. Lett. 552749 (1985).

[60] R. G. Kennett et al., "A Comparison of Diffractive $\Lambda$ and $\Xi^{-}$Photoproduction", Nucl. Phys. B282 626 (1987).

[61] J. A. Appel et al., "Hadron Calorimetry at the Fermilab Tagged Photon Spectrometer Facility", Nucl. Instr. Meth. A243 361 (1986).

[62] B. Zacharov, "CAMAC Systems: A Pedestrian's Guide", Proceedings of the CERN Computing and Data-Processing School, Pertisau (1972).

[63] F. A. Kirsten, "Computer Interfacing for High-Energy Physics Experiments", Ann. Rev. Nucl. Sc. 25 (1975).

[64] P. F. Kunz et al., "The LASS Hardware Processor", SLAC-PUB-2198 (1978). 
[65] P. F. Kunz et al., "Experience using the 168/E Microprocessor for off-line Data Analysis", SLAC-PUB-2418 (1979).

[66] E. Barsotti et al., "A Modular Trigger Processing System for High-Energy Physics Experiments", IEEE Trans. Nucl. Sc. NS-26 686 (1979).

[67] J. Martin et al., "Use of ECL-CAMAC Trigger Processor System for Recoil Missing Mass Triggers at the Tagged Photon Spectrometer at Fermilab", in Proceedings of the Topical Conference on the Application of Microprocessors to High-Energy Physics Experiments, CERN Report 81-07, p. 164 (1981).

[68] T. Nash, "A Review of Programmable Systems associated with Fermilab Experiments", ibid., p. 132.

[69] S. J. Brodsky, F. E. Close, and J. F. Gunion, "Phenomenology of Photon Processes, Vector Dominance, and Crucial Tests for Parton Models", Phys. Rev. D 6177 (1972).

[70] J. D. Bjorken and E. A. Paschos, "Inelastic Electron-Proton and $\gamma$-Proton Scattering and the Structure of the Nucleon", Phys. Rev. 185185 (1975).

[71] H. Fritzsch and P. Minkowski, "Measuring QCD Compton Effects", Phys. Lett. 69B 316 (1977).

[72] V. A. Novikov, M. A. Shifman, A. I. Vainshtein, and V. I. Zakharov, "Quantum chromodynamics and charm photoproduction", Nucl. Phys. B136 125 (1978).

[73] M. Glück and E. Reya, "Duality Predictions for the Production of Heavy Quark Systems in QCD", Phys. Lett. 79B 453 (1978).

[74] L. M. Jones and H. W. Wyld, Jr., "Production of bound quark-antiquark systems", Phys. Rev. D 172332 (1978).

[75] H. Fritzsch and K. H. Streng, "Photoproduction of Heavy Quark Flavours", Phys. Lett. 72B 385 (1978).

[76] J. Babcock, D. Sivers, and S. Wolfram, "Quantum-chromodynamic estimates for heavy-particle production", Phys. Rev. D 18162 (1978).

[77] L. M. Jones and H. W. Wyld, "Charmed-particle production by photon-gluon fusion", Phys. Rev. D 17759 (1978).

[78] C. Leroy and B. Margolis, "Quark Loops, Vector Dominance, and Photon Total Cross Sections", Nucl. Phys. B158 71 (1979).

[79] A. Donnachie, "Baryon Production in Hadron-Hadron Interactions", Z. Phys. C 4161 (1980).

[80] S. F. King, A. Donnachie, and J. Randa, "Diffractive Photoproduction of Quark-Antiquark Jets", Nucl. Phys. B167 98 (1980). 
[81] T. Tung-Sheng, "Photoproduction of Charm and Beauty", Phys. Lett. 89B 232 (1980).

[82] V. Barger, W. Y. Keung, and R. J. N. Phillips, "On $\psi$ and $\Upsilon$ Production via Gluons", Phys. Lett. 91B 253 (1980).

[83] K. Biswal and S. P. Misra, "Charm content of the photon", Phys. Rev. D 24 106 (1981).

[84] M. Fontannaz, B. Pire, and D. Schiff, "Inclusive Photoproduction Cross Sections of Charmed Mesons and Baryons", Z. Phys. C 11211 (1981).

[85] J. G. Körner and G. Kramer, "Weak Decays of Charmed Baryons", Z. Phys. C 2117 (1979).

[86] I. I. Bigi, "Weak Annihilation in Charm Baryon Decays-A Re-evaluation", $Z$. Phys. C 9197 (1981).

[87] I. Bediaga, E. Predazzi, and A. F. S. Santoro, "Search for Diquark Substructures in Charmed Baryon Spectroscopy", Phys. Lett. 105B 71 (1981).

[88] J. dos Anjos, A. Santoro, and M. Souza, "Notes about the $\Lambda_{c}^{+}$", E691 internal memo (unpublished) Fermilab (1985).

[89] M. K. Gaillard, B. W. Lee, and J. L. Rosner, "Search for Charm", Rev. Mod. Phys. 47277 (1975).

[90] T. H. Bauer, R. D. Spital, D. R. Yennie, and F. M. Pipkin, "The hadronic properties of the photon in high-energy interactions", Rev. Mod. Phys. 50261 (1978).

[91] T. Appelquist, R. M. Barnett, and K. Lane, "Charm and Beyond", Ann. Rev. Nucl. Sc. 28 (1978).

[92] D. Treille, "Photon and Hadron Production of Open Heavy Flavours", Proceedings of the 1981 International Symposium on Lepton and Photon Interactions at High Energies, edited by W. Pfeil, p. 750 (1981).

[93] C. A. Heusch, "Heavy Flavour Production from Photons and Hadrons", Proceedings of the SLAC Summer Institute on Particle Physics (1981).

[94] G. H. Trilling, "The Properties of Charmed Particles", Phys. Rep. 7557 (1981).

[95] S. D. Holmes, W. Lee, and J. E. Wiss, "High-Energy Photoproduction of Charmed States", Ann. Rev. Nucl. Part. Sc. 35397 (1985).

[96] A. G. Frodesen, O. Skjeggestad, and H. Tøfte, Probability and Statistics in Particle Physics, Universitetforlaget, Oslo (1979).

[97] I. J. R. Aitchison and A. J. G. Hey, Gauge Theories in Particle Physics, Adam Hilger Ltd, Bristol (1982). 
[98] E. Leader and E. Predazzi, An Introduction to Gauge Theories and the New Physics, Cambridge University Press, New York (1982).

[99] E. D. Commins and P. H. Bucksbaum, Weak Interactions of leptons and quarks, Cambridge University Press, New York (1983).

[100] T. P. Cheng and L. F. Li, Gauge theory of elementary particle physics, Clarendon Press, New York (1984).

[101] L. H. Ryder, Quantum Field Theory, Cambridge University Press, New York (1985).

[102] D. B. Lichtenberg, Unitary Symmetry and Elementary Particles, 2nd ed., Academic Press, New York (1978).

[103] F. Sauli, "Principles of Operation of Multiwire Proportional and Drift Chambers", CERN Report 77-09 (1977).

[104] R. S. Gilmore, "Particle Identification by Cerenkov and Transition Radiation", Proceedings of the SLAC Summer Institute on Particle Physics (1980).

[105] C. W. Fabjan and H. G. Fischer, "Particle Detectors", Rep. Prog. Phys. 43 1003 (1980).

[106] W. W. M. Allison and P. R. S. Wright, "The Physics of Charged Particle Identification: dE/dX, Čerenkov, and Transition Radiation", CERN preprint (1984).

[107] G. Charpak and F. Sauli, "High-Resolution Electronic Particle Detectors", CERN Report 84-35 (1984).

[108] C. W. Fabjan, "Calorimetry in High-Energy Physics", CERN Report 85-54 (1985).

[109] J. E. Augustin et al., "Discovery of a Narrow Resonance in $e^{+} e^{-}$Annihilation", Phys. Rev. Lett. 331406 (1974).

[110] J. J. Aubert et al., "Experimental Observation of the Heavy Particle J", ibid. 1404.

[111] J. D. Bjorken and S. L. Glashow, "Elementary Particles and SU(4)", Phys. Lett. 11255 (1964).

[112] S. L. Glashow, J. Iliopoulos, and L. Maiani, "Weak Interactions with LeptonHadron Symmetry", Phys. Rev. D 21285 (1970).

[113] A. De Rújula, H. Georgi, and S. L. Glashow, "Hadron masses in a gauge theory", Phys. Rev. D 12147 (1975).

[114] J. R. Sanford, "The Fermi National Accelerator Laboratory", Ann. Rev. Nucl. Sc. 26151 (1976). 
[115] P. D. B. Collins and A. D. Martin, "Hadron reaction mechanisms", Rep. Prog. Phys. 45335 (1982).

[116] J. Gasser and H. Leutwyler, "Quark Masses", Phys. Rep. 8777 (1982).

[117] M. Banner et al., "Observation of Single Isolated Electrons of High Transverse Momentum in Events with Missing Transverse Energy at the CERN $p \bar{p}$ Collider", Phys. Lett. 122B 476 (1983); UA1 Collab., G. Arnison et al., "Experimental Observation of Lepton Pairs of Invariant Mass around $95 \mathrm{GeV} / \mathrm{c}^{2}$ at the CERN SPS Collider", ibid. 126B 398 (1983); UA1 Collab., G. Arnison et al., "Further Evidence for Charged Intermediate Vector Bosons at the SPS Collider", ibid. 129B 273 (1983).

[118] M. B. Green, "Unification of forces and particles in superstring theories", $\mathrm{Na}$ ture 314409 (1985).

[119] F. James and M. Roos, "MINUIT-a system for function minimization and analysis of the parameter errors and correlations", Comp. Phys. Comm. 10 343 (1975).

[120] J. Orear, "Least squares when both variables have uncertainties", Am. J. Phys. 50912 (1982).

[121] T. J. Chapin et al., "Diffraction dissociation of photons on hydrogen", Phys. Rev. D 3117 (1985).

[122] T. Sjöstrand, "The LUND Monte Carlo for Jet Fragmentation and $e^{+} e^{-}$Physics: JETSET Version 6.2", Comp. Phys. Comm. 39347 (1986).

[123] D. Blodgett, "Track Quality Study", E516 internal memo (unpublished) (1983).

[124] P. Estabrooks, "Aging Effects in a Large Drift Chamber in the Fermilab Tagged Photon Spectrometer", E691 internal memo (unpublished) (1986).

[125] Z. Koba, H. B. Nielsen, and P. Olesen, "Scaling of Multiplicity Distributions in High Energy Hadron Collisions", Nucl. Phys. B40 317 (1972).

[126] PLUTO Collab., Ch. Berger et al., "Multiplicity Distributions in $e^{+} e^{-}$Annihilations at PETRA Energies", Phys. Lett. 95B 313 (1980).

[127] D. C. Carey and D. Drijard, "Monte Carlo Phase Space with Limited Transverse Momentum", J. Comp. Phys. 28327 (1978).

[128] V. K. Bharadwaj et al., "A Large Area Liquid Scintillation Multiphoton Detector", Nucl. Instr. Meth. 228283 (1985).

[129] M. Macdermott, "A Review of Charm Hadroproduction", in the Proceedings of the Hadronic Session of the Twentieth Rencontre de Moriond (1985), edited by J. Tran Tranh Van. 
[130] Papers submitted by the Tagged Photon Spectrometer Collaboration to the XXIII International Conference on High Energy Physics, Berkeley, California, July 1986:

(i) J. C. C. dos Anjos et al., "Photoproduction of $J / \psi$ Mesons on $\mathrm{H}, \mathrm{Be}$, and $\mathrm{Pb}$ Targets."

(ii) J. C. C. dos Anjos et al., "Lifetime Measurements for the $D^{+}, D^{0}, F^{+}$."

(iii) J. C. C. dos Anjos et al., "Early Results on Charm Photoproduction."

(iv) J. C. C. dos Anjos et al., "New Charm Results using High Precision Vertex Detectors", presented by R. J. Morrison for the TPS Collaboration.

[131] S. Menary, "A Study of the Transverse Momentum Distribution of Photoproduced Charged and Neutral D Mesons", M. Sc. thesis, University of Toronto, Toronto (1986). 


\section{Afterword \\ Research Contribution}

Experiments in high energy physics are impressive undertakings in which the combined efforts of many engineers, technicians, and physicists are required to uncover the subtle, secret messages sown within the very fabric of this universe, our home. Hence this brief afterword has been written with the express purpose of enlightening the concerned reader as to the specific contributions made by the author for the collaboration.

The author joined the E516 collaboration in the fall of 1980 after the construction of the detectors had already been completed. At the time, a preliminary "shakedown" run of the spectrometer had been completed in the spring of that year. The results gave indications of where helpful improvements could be made to the spectrometer system. I was given the task of installing one of these helpful additions.

It was realized that most of numerous high and low voltage power supplies used to power the detectors could (and should) be monitored by the computerized datalogging system. Hence the author was assigned the job of installing the hardware required for this monitoring system. Most of the work entailed the stringing of coaxial cables from the appropriate devices up to the counting room where the data-logging electronics were situated. A total of 76 channels were fed into three 32-channel LeCroy Model $2232 \mathrm{ADC}$ modules for subsequent digitization. This data was both written to tape and monitored online so that the experimenters could react immediately to any power failures.

After the end of the experimental run in June of 1981, the author became involved with several projects concerned with the data reconstruction effort. The 
first project involved the calibration of the Outriggers using several muon runs. I gathered a selection of events that had at least one well defined muon track passing through one of the Outriggers. This data base was utilized by John Martin in his calibration of this detector. In the second project, I helped in the development and running of the $168 \mathrm{E}$ emulator system. This was entirely a local effort by the University of Toronto portion of the E516 collaboration. Section 2-4-12 describes this emulator system and its role in the data reconstruction effort. Thirdly, when the Data Summary Tapes were finally being prepared, I took responsibility over the data unpacking program which everyone would use in their analysis of the data. Although the original version was written at Fermilab, it was suggested by Steve Bracker that a structured version would be more useful over the long run for ease of maintenance and modification. Thus I transformed the Toronto version of this important program into the structured format of FORTRAN-77.

Finally, most of my efforts were directed to analyzing the E516 data sample in order to find and study charmed baryons. Hence the work presented in Chapter 3 is entirely my own effort. During the time of my analysis of the data, parallel efforts were being made in California [60] and Colorado [49] in a similar direction to mine. It must be understood that my analysis was and is completely independent of these other projects. This is not meant to be in any way a comment on the quality of these other analyses, but simply a statement of the uniqueness and individuality of the author's research effort. At the same time, this work could not have been executed without the prior efforts of the other members of the E516 collaboration. I wish to thank them once again. 